\title{
DEPOSITION MODELING OF HIGH DENSITY TAILINGS USING SMOOTHED PARTICLE HYDRODYNAMICS
}

\author{
A thesis submitted to \\ the Faculty of Graduate and Postdoctoral Affairs \\ in Partial Fulfillment of the requirements for the degree \\ Masters of Applied Science
}

by

Yagmur Babaoglu

B. Eng., Carleton University, 2011

\author{
Department of Civil and Environmental Engineering \\ Carleton University \\ Ottawa-Carleton Institute of Civil and Environmental Engineering \\ January 2014 \\ C)Yagmur Babaoglu
}




\begin{abstract}
High density (HD) tailings are tailings that have been sufficiently dewatered, where they exhibit a yield stress upon deposition, and therefore naturally form gently sloped deposits that do not requires dams for containment. It is essential to comprehend and model the flow behaviour during deposition to predict the final geometry of the stack and control storage capacity; which are important design elements to HD tailings technology. As HD tailings exhibit a yield stress, modelling stack geometry constitutes, in part, a problem of non-Newtonian flow with a free surface.

This research investigated modelling the flow behaviour of HD tailings, using an open-source Smoothed Particle Hydrodynamics (SPH) code. The results indicated that two-dimensional simulations using SPH agreed well with experimental data for single and multi-layer flume tests. SPH has the advantage over simpler methods, such as Lubrication Theory, as SPH better predicts the geometry when inertia influences the flow of tailings.
\end{abstract}




\section{Acknowledgements}

I would like to thank my thesis supervisor Dr. Paul Simms for his support, guidance and patience during the course of my thesis. He inspired and helped me in every step of my research and I would not be able to complete this work without him.

I would also like to thank Mr. Stanley Conley, Mr. Jason Arnott, Mr. Pierre Trudel and Ms. Marie Tudoret Chow for their kindness to provide me hours of technical support. I would like to thank Ms. Payal Chadha for her kind assistance.

Finally, I would like to thank to my family, my boyfriend and my friends for their constant encouragement and support. 


\section{Table of Contents}

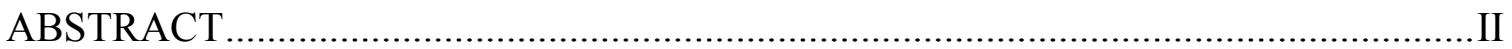

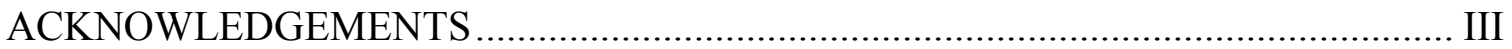

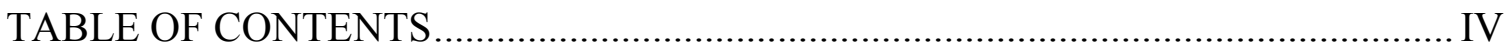

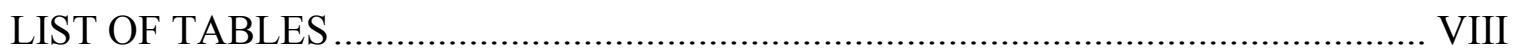

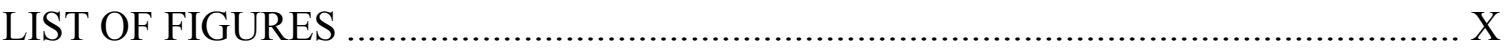

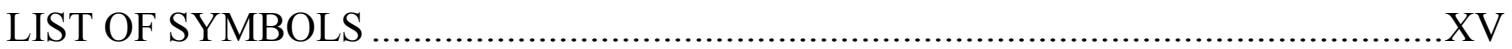

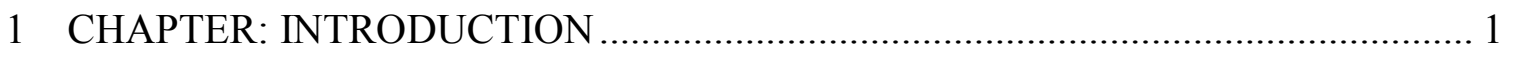

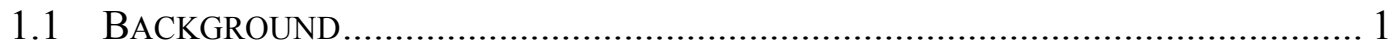

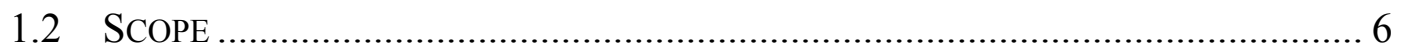

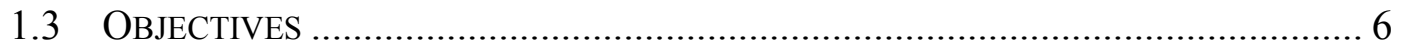

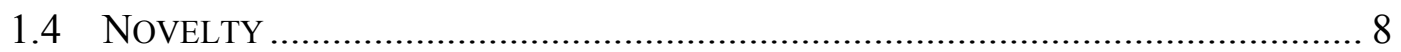

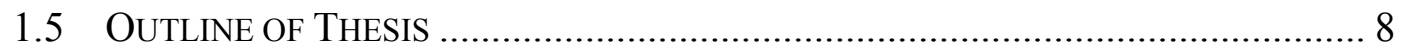

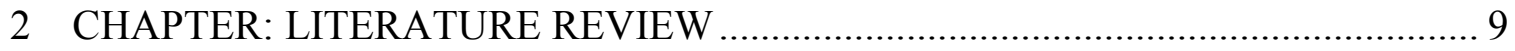

2.1 Definition of TAILING ANd Conventional Deposition …………............... 9

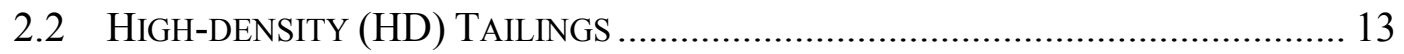

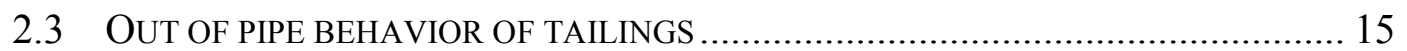

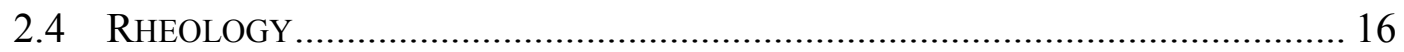

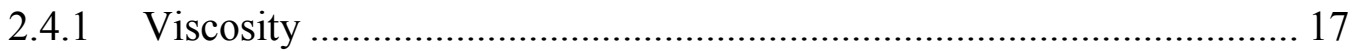

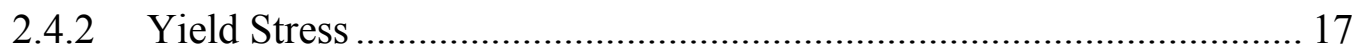




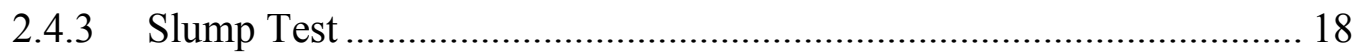

2.5 Complications of Non-Newtonian Stress-Strain Relation ................. 19

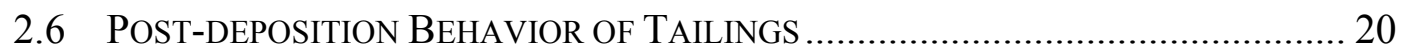

2.7 Prediction of Deposition Geometry ……….......................................... 22

2.7.1 Fitton's Beach Slope Method................................................................ 23

2.7.2 McPhail's Stream Power Method ...................................................... 24

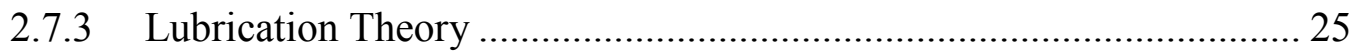

2.8 NumERICAL ModELING OF Non-NEWTONIAN FLUIDS ................................... 27

2.8.1 Finite Volume Method ................................................................... 28

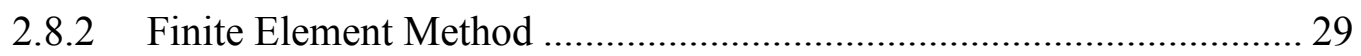

2.8.3 Smoothed Particle Hydrodynamics (SPH)........................................ 30

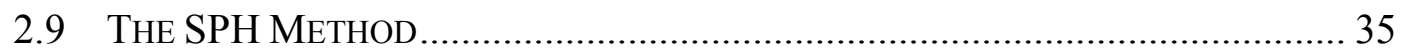

2.9.1 General Formulation of SPH ………………….............................. 36

2.9.2 Boundary Conditions ...................................................................... 40

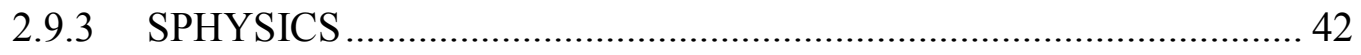

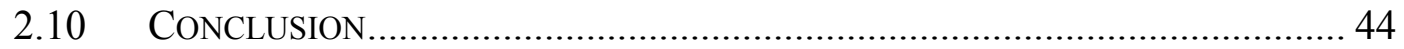

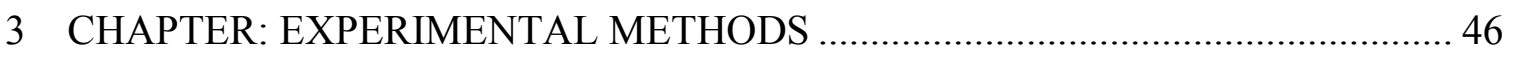

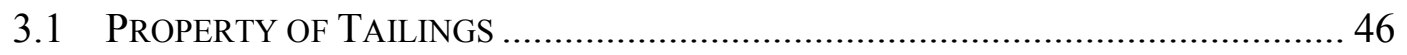

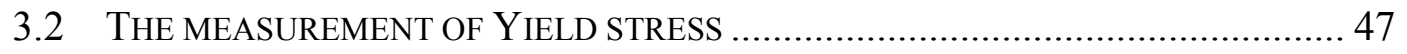

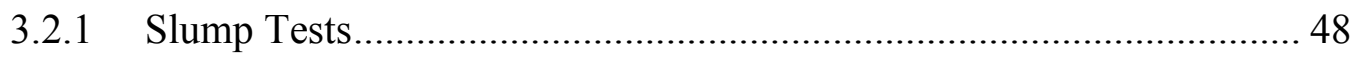

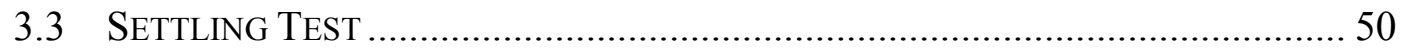

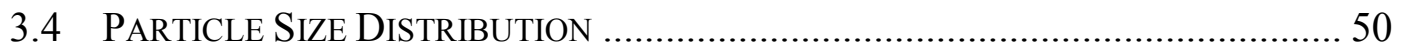




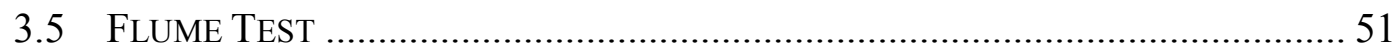

3.5.1 Experimental Procedure ................................................................... 52

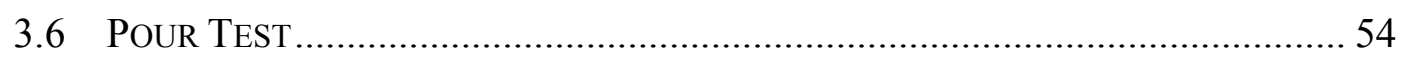

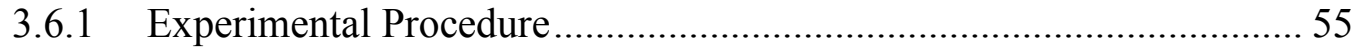

4 CHAPTER: EXPERIMENTAL RESULTS AND DISCUSSION ………………...... 57

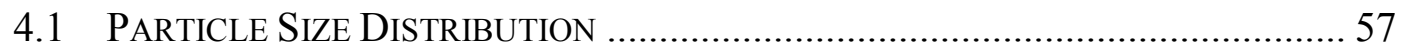

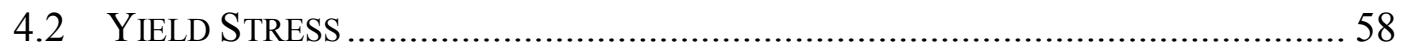

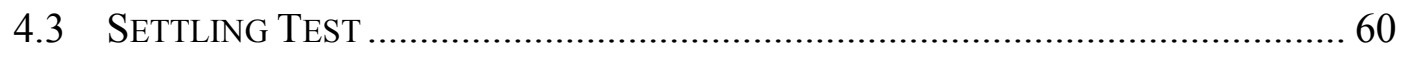

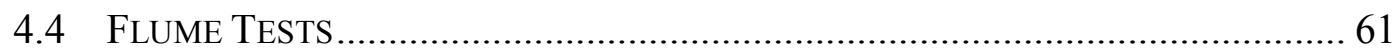

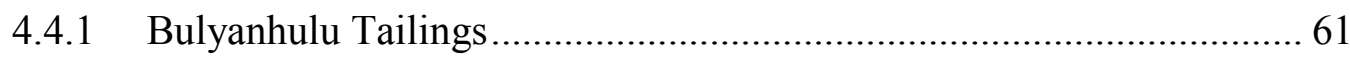

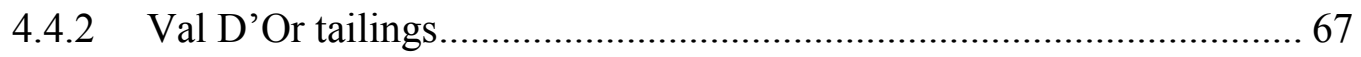

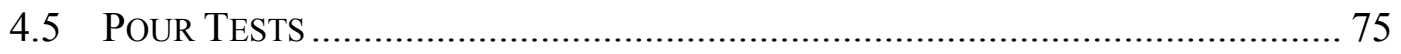

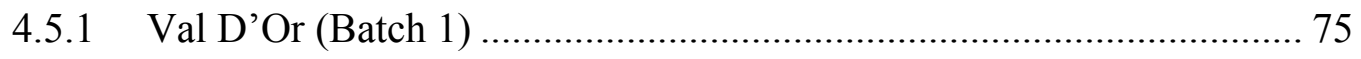

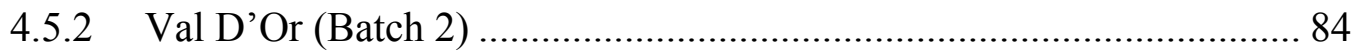

5 CHAPTER: NUMERICAL ANALYSIS AND RESULTS ……………………........ 90

5.1 IMPLEMENTATION AND FORMULATION IN SPHYSICS .................................... 90

5.1.1 Rheological Model...................................................................... 90

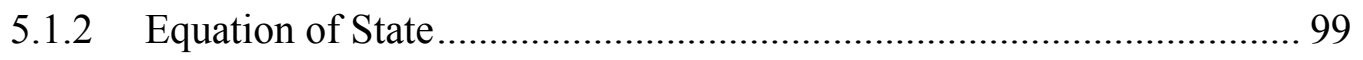

5.1.3 Density Re-Initialization.................................................................. 103

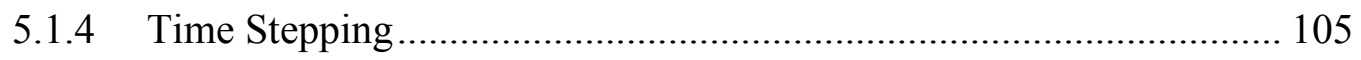

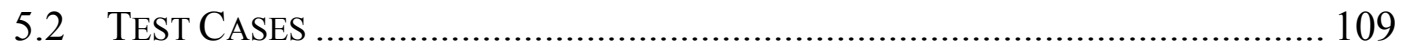

5.2.1 Single Layer Flume Tests ................................................................ 110 
5.2.2 Multi-Layer Flume Test.

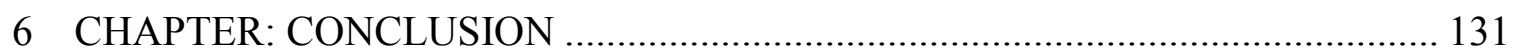

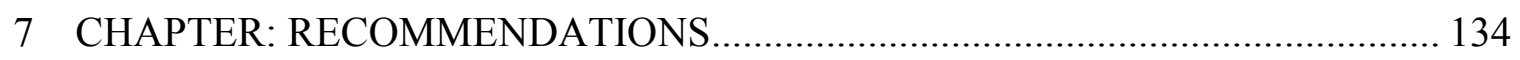

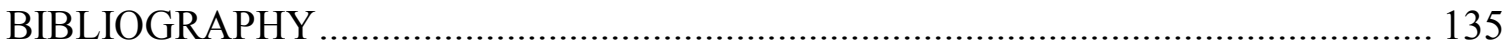

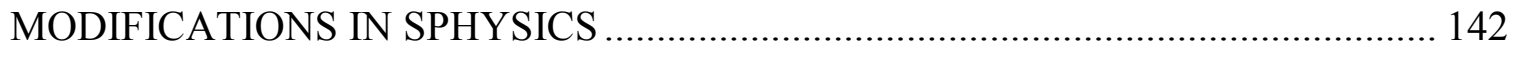

A.1 Modifications in Generator Code...................................................... 142

A.2 Modifications in SPHYSICS Code ………………............................ 144 


\section{List of Tables}

Table 2.1: Rheological characteristics of various thickened tailings (Jewell and Fourie 2006) 9

Table 2.2: Different compiling options used in SPHYSICS (Gómez-Gesteira, et al. 2010).

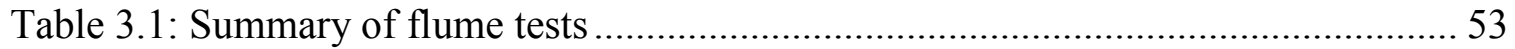

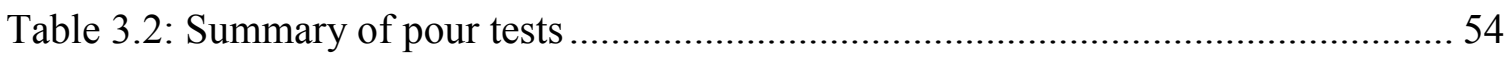

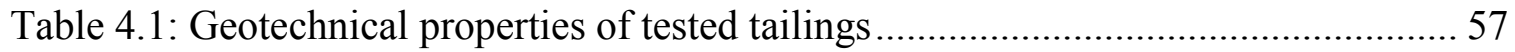

Table 4.2: Summary of flume tests conducted with Bulyanhulu tailings. ....................... 62

Table 4.3: Best-fitted yield stress values obtained from LT equations for Bulyanhulu

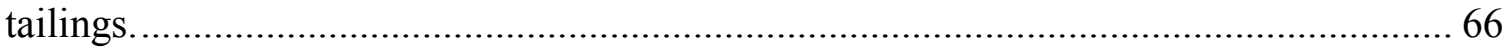

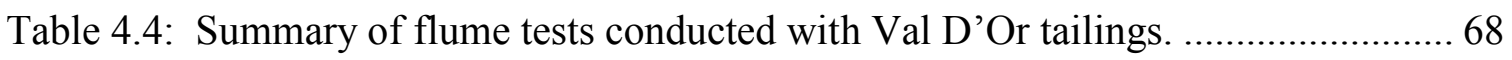

Table 4.5: Summary of flume tests conducted with material at $51-52 \%$ water content ... 70

Table 4.6: Summary of flume test conducted with material at 55\% gravimetric water

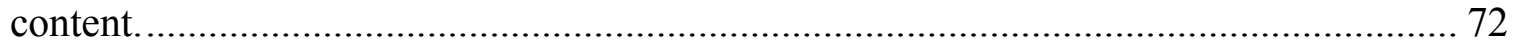

Table 4.7: The summary of best fitted yield stress values based on LT....................... 74

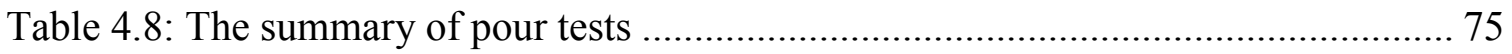

Table 4.9: Rheological properties of Val D'Or tailings for each layer for Pour test \#1 ... 76

Table 4.10: Best fitted yield stress values for Pour test \#1 ......................................... 79

Table 4.11: Rheological properties of each Val D’Or tailings layer in Pour test \#2. ...... 81

Table 4.12: The rheological properties of each layer for Pour test \#3............................ 84

Table 4.13: Best-fitted yield stress results calculated using Equation 3.3 and 3.4 ......... 87 
Table 5.1: The summary of numerical experiments conducted using SPH.

Table 5.2: The key parameters chosen for single layer deposition of Bulyanhulu high

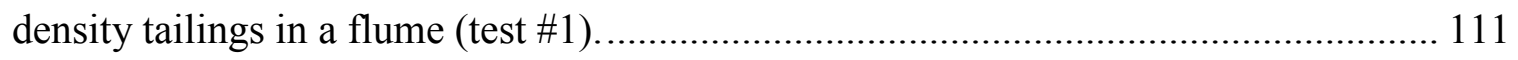

Table 5.3: The key SPH parameter chosen for large volume tests using a funnel and a gate with yield stress and viscosity values $36 \mathrm{~Pa}$ and $0.65 \mathrm{~Pa} . \mathrm{s}$ respectively.............. 115

Table 5.4: The parameters selected for test \#4. ....................................................... 118

Table 5.5: Parameters selected for the flume tests on a sloping bed. ......................... 121

Table 5.6: Parameters selected for multi-layer simulation. ....................................... 125

Table 5.7: Parameters chosen for multi-layer simulation with smaller $\tau_{\mathrm{y}}$ and larger $\alpha$ value. 128 


\section{List of Figures}

Figure 1.1: Surface depositions of tailings in Bulyanhulu mine (Theriault, et al. 2003).... 3

Figure 1.2: Channelizing Flow (with permission of Musselwhite Mine) .......................... 5

Figure 2.1: Embankment types (a) Upstream. (b) Down-stream or water-retention (c)

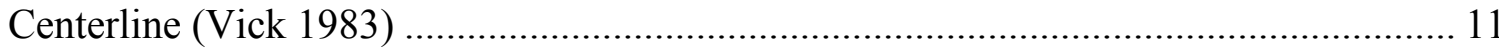

Figure 2.2: Los Frailes Dam failure (http://www.tailings.info/casestudies/losfrailes.htm)

Figure 2.3: Thickened tailings running of the stack initially as the channeling flow (Mizani, et al. 2013). 16

Figure 2.4: Mud front at different times $(\mathrm{t}=12,16,20,24,28,32,36$ and $38 \mathrm{~s}$ (Hosseini, et al. 2007). 33

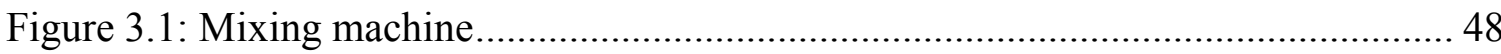

Figure 3.2: Open ended PVC cylinder with $19 \mathrm{~cm}$ height ............................................... 48

Figure 3.3: The schematic diagram of slump test (Pashias, et al. 1996)........................... 49

Figure 3.4: Flume apparatus …………………………......................................... 51

Figure 3.5: The pump

Figure 3.6: Plexiglas box with plastic sheet cover on top............................................. 55

Figure 4.1: Hydrometer test results for the Bulyanhulu and Val D'Or tailings (material

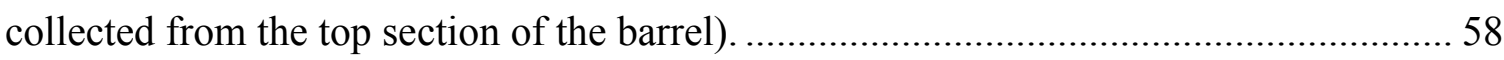

Figure 4.2: Dimensionless slump height versus water content.......................................... 59

Figure 4.3: Yield stress values versus gravimetric water content...................................59 
Figure 4.4: Six single layer flows at different flow rates with volumes approximately 11 $\mathrm{L}$ (gravimetric water content is $37 \%$, yield stress is $71.47 \mathrm{~Pa}$ ). 63

Figure 4.5: The final profile of Bulyanhulu tailings at 38\% water content with a flow rate of 0.25 LPM including LT prediction. 64 Figure 4.6: Single layer test with a deposition rate of 2.5 LPM compared with LT prediction. 64

Figure 4.7: The final profile of tailings with a flow rate of 4.3 LPM including LT prediction. 65

Figure 4.8: Three single layer flows with volumes approximately 9 L (gravimetric water content is $38 \%$ and yield stress is approximately $50 \mathrm{~Pa}$ ) 66

Figure 4.9: Best-fitted yield stress versus deposition time for Bulyanhulu tailings. 67 Figure 4.10: Final profile of Val D'Or tailings with 53\% gravimetric water content and roughly $60 \mathrm{~Pa}$ of yield stress. 69 Figure 4.11: The comparison of predicted and the measured final profiles of Val D'Or tailings with a flow rate of $1.58 \mathrm{LPM}, 53 \%$ gravimetric water content and $66 \mathrm{~Pa}$ of yield stress. 70

Figure 4.12: Final geometry of the Val D'Or tailings with different flow rates (at 52\% water content) 71

Figure 4.13: Best-fitted yield stress values versus deposition time for 52\% water content. .71

Figure 4.14: The comparison between the predicted and measured final profiles of the tailings (Val D’Or Batch \#1) at 52\% water content (Flow rate $\left.=2.28 \mathrm{LPM}, \tau_{\mathrm{y}}=80 \mathrm{~Pa}\right) .72$ 
Figure 4.15: Final profiles of the tests summarized in Table 4.6 with 55\% water content.

Figure 4.16: The comparison of predicted and measured final geometry of material with $55 \%$ gravimetric water content and 2.75 LPM deposition rate. 73

Figure 4.17: The comparison of predicted and measured final geometry of material with $44.5 \%$ gravimetric water content and 1.62 LPM deposition rate. 74

Figure 4.18: Geometry of each layer (1-4) deposited for the first pour tests with 0.28

LPM flow rate. 77

Figure 4.19: Final geometry measured from the experiment for Pour test \#1with 0.28 LPM flow rate (North - South direction) 78

Figure 4.20: The final predicted geometry for Pour tests \# 1 with 0.28 LPM flow rate. . 78 Figure 4.21: Comparison between the final geometry and predicted geometry using bestfitted yield stress values for Pour test (North direction). 79

Figure 4.22: The failures in settled second layer in the directions of (a) North-South (b)

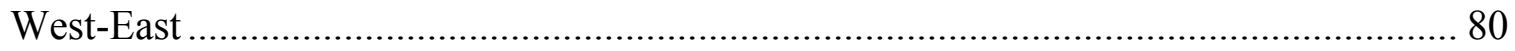

Figure 4.23: The measured final geometry for Pour test \#2 (North- South direction) ..... 82 Figure 4.24: The predicted final geometry based on Lubrication equations for pour test \#2 82

Figure 4.25: The failure in settled layers in Pour tests \#2 (North-South Direction)........ 83

Figure 4.26: The measured topography of Val D'Or tailings of pour test \#3 in (a)NorthSouth and West-East directions (3.7 LPM deposition rate and 1.3 L of volume) .......... 85

Figure 4.27: The final geometry of Val D'Or tailings, plan view after 5 layers. ............ 86

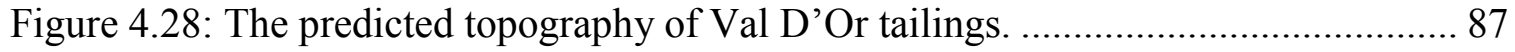


Figure 4.29: Predicted topography using best-fitted yield stress values (South direction) 88

Figure 5.1: The sensitivity of the model to various inputs (a) $\tau_{\mathrm{y}}=36 \mathrm{~Pa} . \mu=0.65 \mathrm{~Pa} . \mathrm{s}$ $\alpha=40$ (b) $\tau_{\mathrm{y}}=36$ Pa. $\mu=1.5$ Pa.s $\alpha=40$ (c) $\tau_{\mathrm{y}}=72$ Pa. $\mu=0.65$ Pa.s $\alpha=40$ (d) $\tau_{\mathrm{y}}=36 \mathrm{~Pa}$. $\mu=0.65$ Pa.s $\alpha=100$ 94

Figure 5.2: The effect of inertia demonstrated for different initial conditions for the same volume of tailings (a) h=0.1 m (b) $0.2 \mathrm{~m}$ (c) $0.3 \mathrm{~m}$ (d) $0.4 \mathrm{~m}$ (e) $0.8 \mathrm{~m}$. 95

Figure 5.3: Final profile of tailings with various number of particles (476, 357 and 236 respectively) 98

Figure 5.4: Sensitivity analysis of the proposed model to time stepping. 99

Figure 5.5: Initial particle positions for 'Fill the cup' tests 101

Figure 5.6: Pressures vs. Particle heights for Newtonian fluid. 101

Figure 5.7: Pressure vs. Particle height for Non-Newtonian fluids $(\rho=1823.8 \mathrm{~kg} / \mathrm{m} 3$ \& $\tau_{\mathrm{y}}=45 \mathrm{~Pa}$ ) (a) $c_{0}$ is $10 \mathrm{~m} / \mathrm{s}$ and the viscosity is $0.5 \mathrm{~Pa} . \mathrm{s}$ (b) $c_{0}$ is $14 \mathrm{~m} / \mathrm{s}$ and the viscosity is 0.8 Pa.s. 102

Figure 5.8: Density field for 2-D dam break showing effect of density (a) without density filter (b) with First order-MLS filter (Crespo 2008). 105

Figure 5.9: Domain divided into $2 \mathrm{~h}$ cells and the particle only interacts with the neighbouring particle marked by black dots (Gómez-Gesteira, et al. 2010). 108

Figure 5.10: The code sweeping through each cell starting from left corner (GómezGesteira, et al. 2010). 109

Figure 5.11: The initial particle and boundary positions for test \#1..... 111

Figure 5.12: The final shape of the tailings at the end of the deposition. 112 
Figure 5.13: The shape of free surface at various times (a) $t=3.5 s$. (b) $t=7.0 \mathrm{~s}$ (c) $\mathrm{t}=10.5 \mathrm{~s}(\mathrm{~d}) \mathrm{t}=14 \mathrm{~s}$

Figure 5.14: The initial position of the particles for the experiment \#2 where the initial height of the particles is $0.2 \mathrm{~m}$ and the length is $0.18 \mathrm{~m}$. 115

Figure 5.15: The final geometry of the experiment \#2. 115

Figure 5.16: The geometry and the initial particle positions for test $\# 3$ (a) $t=0 \mathrm{~s}$ (b) $t=2.0$ S. 116

Figure 5.17: The final geometry of the fluid for test \#3. 117

Figure 5.18: The initial boundaries and particle positions for the first part of the simulation $(3 \mathrm{~s})$ 118

Figure 5.19: Measured and predicted flow profiles at 3-6-9-11 seconds.

Figure 5.20: Measured and predicted flow profiles for smaller B value at 1.3-2.6-3.9-6.2 seconds.

Figure 5.21: Predicted and measured flow profiles at a slope of 0.57 degrees. 122

Figure 5.22: Predicted and measured flow profiles at a slope of 1.14 degrees. 122

Figure 5.23: The final flow profile for the first layer. 123

Figure 5.24: Final flow profiles for various $\alpha$ and $\tau_{y}$ values at 2.4 seconds (a) $\alpha=5000$, $\tau_{\mathrm{y}}=100$ (b) $\alpha=40, \tau_{\mathrm{y}}=400$ (c) $\alpha=5000, \tau_{\mathrm{y}}=400$ (d) $\alpha=50000, \tau_{\mathrm{y}}=1000$ 125

Figure 5.25: Flow profiles at $\mathrm{t}=0.01 \mathrm{~s}, \mathrm{t}=1.0 \mathrm{~s}, \mathrm{t}=2.0 \mathrm{~s}, \mathrm{t}=3.0 \mathrm{~s}, \mathrm{t}=5.0 \mathrm{~s}$ 127

Figure 5.26: Flow profiles at $\mathrm{t}=0.01 \mathrm{~s}, \mathrm{t}=1.0 \mathrm{~s}, \mathrm{t}=2.0 \mathrm{~s}, \mathrm{t}=3.0 \mathrm{~s}, \mathrm{t}=5.0 \mathrm{~s}$. 130 


\section{List of Symbols}

c

$\mathrm{C}_{\mathrm{S}}$

d

D

e

g

$\mathrm{h}$

$\mathrm{H}$

$\mathrm{H}_{\mathrm{E}}$

$\mathrm{h}_{\mathrm{p}}$

i

K

$\mathrm{K}^{\mathrm{eff}}$

$\mathrm{m}$

$\mathrm{P}$

$\mathrm{P}_{\mathrm{S}}$

q

Q

r

$\mathrm{R}_{\mathrm{H}}$

$S^{\prime}$
Speed of sound

Solid concentration per volume

Depth of flow

Shear rate tensor

Energy

Gravitational acceleration

Smoothing length

Depth

Energy head

Distance between points

Channel bed slope

Consistency coefficient

Coefficient in the artificial viscosity term

Mass

Pressure

Stream power

Non-dimensional distance

Flow rate

Particle position

Hydraulic radius

Dimensionless yield stress 


\begin{tabular}{|c|c|}
\hline $\mathrm{S}_{\mathrm{B}}$ & Stream power curve \\
\hline$v$ & Velocity \\
\hline V & Critical velocity \\
\hline $\mathrm{V}_{\mathrm{x}}$ & Velocity head \\
\hline $\mathrm{v}_{\mathrm{sig}}$ & Signal velocity \\
\hline W & Weighting function \\
\hline$\alpha$ & Coefficient for artificial viscosity term \\
\hline$\alpha_{D}$ & Normalization constant \\
\hline$\alpha_{\mu}$ & Dynamic viscosity \\
\hline$\ddot{\Upsilon}$ & Shear rate \\
\hline$\eta$ & Corrective term \\
\hline$\eta_{(\ddot{O})}$ & Plastic viscosity \\
\hline$\theta$ & Angle of inclined surface \\
\hline$\theta_{r}$ & Overall angle \\
\hline$\mu$ & Dynamic viscosity \\
\hline$\Pi$ & Viscosity term \\
\hline$\rho$ & Density \\
\hline$\tau$ & Shear stress \\
\hline$\tau_{y}$ & Yield stress \\
\hline$\tau_{y}^{\prime}$ & Dimensionless yield stress \\
\hline$\Psi_{\mathrm{ab}}$ & Viscosity term in energy equation \\
\hline
\end{tabular}




\section{Chapter: Introduction}

\subsection{Background}

The mining industry makes a great contribution to Canada's economy. The industry employs approximately 320,000 workers across the country and contributes $\$ 35.6$ billion to the country's Gross Domestic Product (GDP) (MAC 2012). Massive volumes of waste materials, such as tailings and waste rock, are produced annually by mining operations, creating some of the largest earth structures in the world. The risks of improper management of these structures increase in the public's consciousness every day. One of the best examples is the tailing storage facilities in Northern Alberta, which are some of the few man-made structures in the world that are distinguishable from the orbit of the International Space Station.

Mining can be described as the extraction of valuable minerals from the ore body. The process involves excavation of the subsurface material, which is later on crushed, grinded and/or processed in order to extract the economic fraction, resulting in massive amounts of waste material generated commonly known as waste rock and mine tailings. Waste rock is the rock left over after blasting and excavation during the normal course of mining, and varies in size from boulders to fine sand. Mine tailings are crushed rock or fine soil particles generated or deposited in slurry form and their size varies from fine sand to silt size particles (Vick 1983). 
Waste rock is deposited in piles in unconfined areas and is associated with environmental problems, such as acid rock drainage (Akcil and Koldas 2006). Historically, mine tailings were deposited, where it was the most cost-effective and convenient; valleys or directly into water bodies. However, current practices involve various types of deposition techniques such as disposal of tailings in slurry form in dammed impoundments, backfilling underground mine and open-pits, subaqueous deposition and disposal of dry or high density tailings in impoundments or self-standing piles (Vick 1983).

The most common type of deposition for tailings as a slurry confined by engineered surface impoundments using dams and embankments. Subsequent to deposition of these tailings, coarser particles settle closer to the discharge point, whereas finer particles settle farther away from the deposition point (Vick 1983). The height of these dams can reach up to several hundred feet and the impoundment can cover several square kilometres. However, conventional storage systems pose a high risk of catastrophic dam failure due to several factors; earthquakes, poor construction techniques, poor water control in the pond and associated high pore pressure. Such failures can result in severe economic and environmental damages and even in loss of life. For example, the failure of a alumina tailings impoundment in Hungary cost the life of 7 people while injuring 120 others in 2010 (Herard 2010).

High density tailings (HD) was developed in order to reduce the need of dams for containment and to increase water recycled during operation. HD tailings are 
dewatered by some process before deposition to the point that they manifest a yield stress upon deposition.

The first attempt to produce HD tailings was made under the guidance of Dr. E. Robinsky in 1973 at the Kidd Creek Mine in Canada. Thickened tailing disposal (TTD) technology involves dewatering of high density tailings to a point, where grain size segregation will not occur upon deposition.

Thickened and paste tailings are both dewatered and difficult to differentiate. However, there is some dissimilarity such as; paste tailings do not have a critical flow velocity when pumped, and therefore may be transported by laminar flow. To minimize the settling during transport, they require a certain fine fraction, a rule of thumb of $20 \%$ less than 15 microns (Cincilla, et al. 1997; Theriault, et al. 2003).
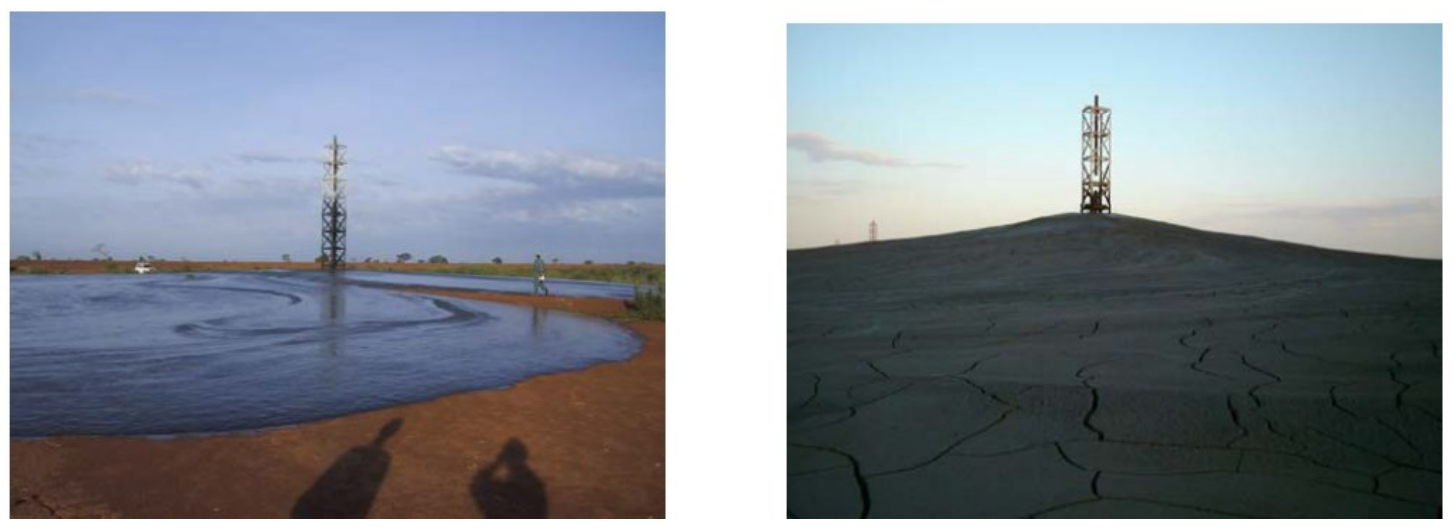

Figure 1.1: Surface depositions of tailings in Bulyanhulu mine (Theriault, et al. 2003)

Bulyanhulu gold mine, in Tanzania, Africa is one of the well-known example adopting a full scale surface disposal of paste tailings (Shuttleworth, et al. 2005). The climate of the area is semi-dry and the main reason to apply this technique there is to conserve water during operation. Figure 1.1 demonstrates one of the 
multiple towers available in the site where the tailings deposition is cycled between them as well as the consolidation of the deposited layer (Theriault, et al. 2003).

The flow of HD tailings away from the deposition point and the estimation of stack geometry are important for the management of these facilities. Understanding the behavior of HD tailings is crucial for the estimation of the stack geometry and overall slope angle. These properties have an influence on the determination of the storage capacity and geotechnical stability of the stack. For example, if the overall slope angle is too steep; then it promotes erosion and decreases the geotechnical stability of the stack. On the contrary, if the angle is too small, impoundment footprint is increased as a result.

The deposition behavior of HD tailings received less attention in literature compared to the behavior of high density tailings in the pipe. A single slope, measured at laboratory scale, was used to characterize the HD tailings stack. However, it is now proven that the slope of the deposit in the field is significantly smaller than the slope at the laboratory scale. The overall slope angle is dependent on the scale of the deposition. For example, smaller or younger deposits uniformly spread on the impoundment bed when they deposited and form a convex beach slope profile. In older or larger deposits, the tailings may flow in narrow channels for a substantial fraction of the beach length and overall beach slope profile is concave (Simms, et al. 2011). There is a debate on what are the reasons for the concave behaviour. Some researchers believe concavity to arise from the flow behaviour, while others believe it is only a function of the variability in rheology of the tailings produced by the thickener (Simms, et al. 2011). 


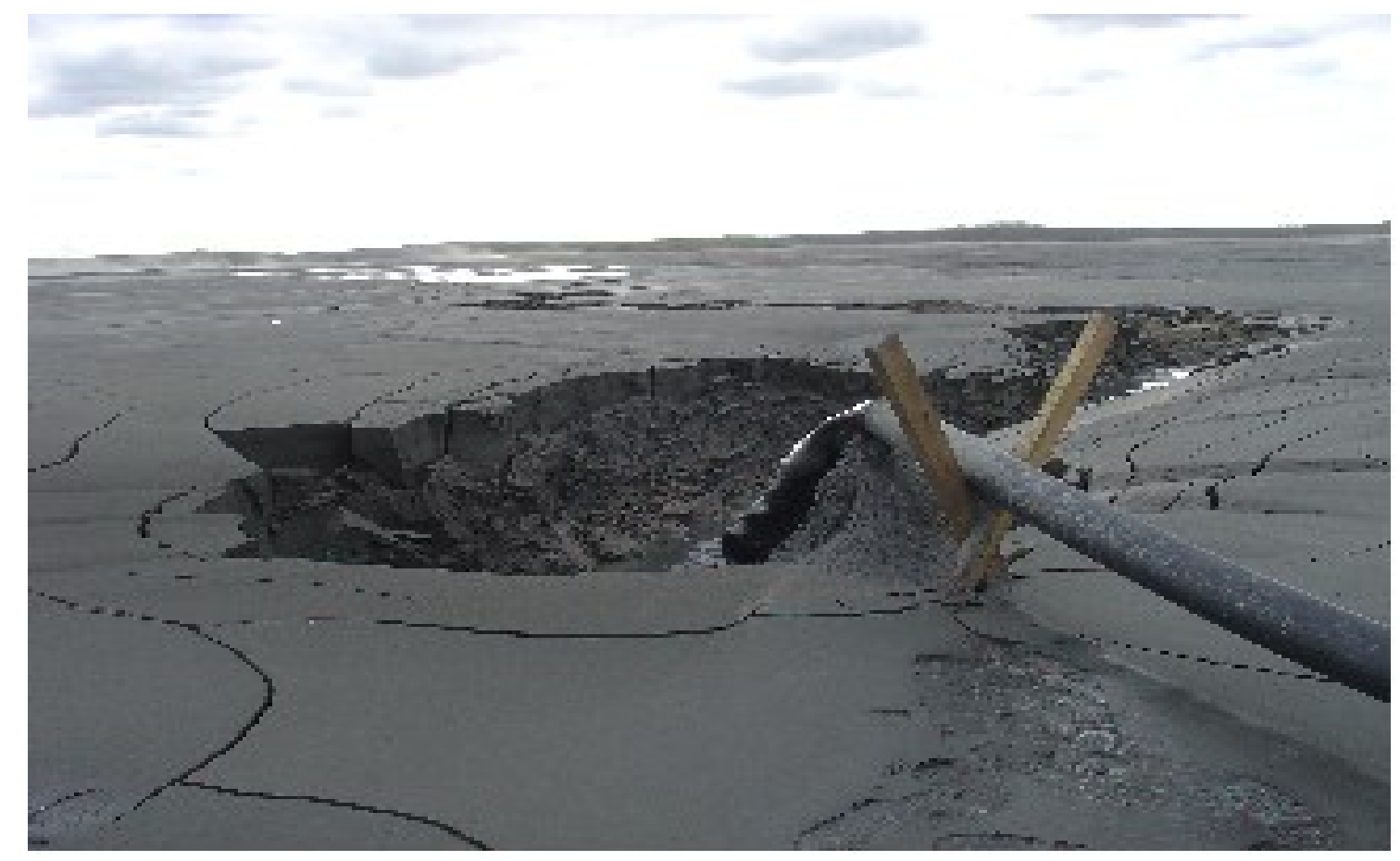

Figure 1.2: Channelizing Flow (with permission of Musselwhite Mine)

A variety of theories has been investigated in the literature for the prediction of equilibrium beach profiles. Fitton's beach slope method (Fitton, et al. 2006) and McPhail's stream power method (McPhail, et al. 2008) are based on the analysis of channelized flow developed during deposition. Lubrication Theory investigates the prediction of equilibrium profiles for spreading tailings flow (Simms, et al. 2011). Tailings may undergo different stages during and before the deposition; laminar or turbulent, supercritical or subcritical, channelized or spreading. Tailings may be in supercritical state when they exit the pipe, going through hydraulic jump close to deposition point, then spread in the surface in subcritical state. Later on the same stack, tailings no longer go through hydraulic jump but channelize instead and then convert to 
spreading flow state near the bottom of the stack as seen in Figure 1.2 (Mizani et al. 2013).

\subsection{Scope}

The scope of this research is to simulate depositional behaviour of high density tailings using Smoothed Particle Hydrodynamics (SPH) at a laboratory scale. The experimental work includes several flume tests and small tests with unconstraint deposition to obtain a better understanding of the tailings flow behaviour.

The simulations are carried by using SPHYSICS program using the 'bi-viscosity' model as the rheological model. The free parameters of the rheological model are determined by comparing the results with the experimental data. The results are compared with the experimental data acquired in the laboratory as part of this thesis, as well as data obtained from Henriquez and Simms (2009), for single and multi-layer depositions to test the applicability of the proposed model. To further investigate the effect of inertia, two different deposition tests are simulated including using a gate and a funnel.

\subsection{Objectives}

The prediction of the overall slope and the geometry of the HD tailings received attention from several researchers where the variety of theories reflects different flow states as tailings flow (Simms, et al. 2011). The present work aims to simulate high density tailings as non-Newtonian fluids in order to predict the final geometry and to 
simulate the different stages of the flow by using Smoothed Particle Hydrodynamics method.

In recent years, significant progress has been achieved by using several numerical methods to study non-Newtonian fluid flows. (Bovet, et al. 2007; Dean, et al. 2007; Glowinski 2011; Yuhi and Mei 2004). One such method is Smoothed Particle Hydrodynamics (SPH). It was originally introduced by Gringold \& Monaghan (1977) and Lucy (1977) to solve problems in astronomical physics and later extended to solve free surface problems in fluid mechanics by Monaghan (1994). Hosseini et al. (2007) used a three-step algorithm, fully explicit in time, to solve incompressible non-Newtonian fluid problems. Shao and Lo (2003) developed an incompressible SPH model to simulate nonNewtonian fluids with free surfaces. Capone et al. (2011) simulated submarine landslides as a non-Newtonian fluid using the SPH method.

SPH based program, SPHYSICS, was selected for the simulation of tailings. SPHYSICS is originally developed for Newtonian fluids; some modifications were required for its applicability to non-Newtonian fluids. The results from the simulations are compared with the experimental data performed using a flume also the data obtained from Henriquez and Simms (2009).

The specific objective of the thesis is to examine the suitability of using SPH for modeling high density tailings. This is done by modelling well-controlled laboratory tests of relatively simple geometries, such as single layer or multi-layer flume tests in two dimensions. 


\section{$1.4 \quad$ Novelty}

The Smoothed Particle Hydrodynamics method has been previously studied by various researches for modelling non-Newtonian fluid problems. Past studies include modelling of rheometry tests, dam break problems for mudflows and debris flows; also the interaction of non-Newtonian fluids with water using SPH. This research specifically investigates the depositional behaviour of high-density tailings including single and successive layer depositions at a laboratory scale.

\subsection{Outline of Thesis}

The outline of the thesis is presented as follows;

Chapter 2: Presents a review of tailings and their management including the rheology of non-Newtonian fluids and different theories related to the prediction of tailings stacks geometry. In addition, analyses of various numerical methods applied to non-Newtonian fluids are discussed in this chapter.

Chapter 3: Experimental methods

Chapter 4: Experimental results and discussions

Chapter 5: Theory behind the SPH method and the modifications applied to SPHYSICS program explained in details. Also, the results from the numerical model is presented and discussed.

Chapter 6: Conclusions

Chapter 7: Presents some recommendations for future work. 


\section{Chapter: Literature Review}

\subsection{Definition of Tailing and Conventional Deposition}

Tailings are the waste product generated from the milling process. The valuable minerals are extracted from the ore using mechanical and chemical processes and produce a waste stream known as tailings. They consist of unrecoverable and uneconomic metals, minerals, chemical and process water. They are normally discharged as slurry to a final storage system also known as Tailings Storage Facility (TSF) (Vick 1983).

The characteristics of tailings are dependent on the original mineralogy of the ore and the particular processing operation. Some examples are presented in Table 2.1.

\begin{tabular}{|l|c|c|c|}
\hline & Coal tailings & Gold tailings & Lead-zinc tailings \\
\hline Specific gravity $\left(\mathrm{kg} / \mathrm{m}^{3}\right)$ & 1450 & 2800 & 4100 \\
\hline Solids concentration $(\% \mathrm{w} / \mathrm{w})$ & 36 & 75 & 75 \\
\hline Slurry Density $\left(\mathrm{kg} / \mathrm{m}^{3}\right)$ & 1120 & 1930 & 2310 \\
\hline
\end{tabular}

Table 2.1: Rheological characteristics of various thickened tailings (Jewell and Fourie 2006)

In order to extract the ore, some chemicals and reagents are used during the milling process and they represent short and long term tailings management challenges. The generation of acid rock drainage (ARD) is for example, is one of the major environmental problems challenging the mining industry. ARD is produced when sulfide-bearing material is subjected to oxygen and water which causes natural oxidations and leading to generation of acids. The factors affecting ARD are highly variable from site-to-site, predicting the potential ARD is highly challenging and costly. Due to the presence of concentrations of heavy metals and other toxic elements, it can severely contaminate the 
surface and the ground water as a result, develop a significant environmental problem (Akcil and Koldas 2006).

The traditional methods of deposition for mine tailings employ dams and embankments of various types to retain slurry tailings and mill effluent. The traditional methods of containment are the use of water-retention type dams or raised embankments. The water-retention dams are constructed to their full height before discharge. They are suitable for all types of tailings and the water storage capacity of these dams is high. Their resistance to seismicity is efficient; however, relative cost of the embankment is significant (Vick 1983).

For raised embankments the construction of the embankments is staged over the life of the impoundment. Raised embankments start with the construction of the starter dike, using natural soil borrow and sized to contain two-to-three years mill tailing and the additional storage for floodwater. There are three main type of raised embankments available; upstream, downstream and centerline (Fig 2.1) (Vick 1983).

The upstream method initially involves the construction of the starter dyke and then the tailings are discharged peripherally to form a beach. The beach becomes the foundation of the second perimeter dyke and the height of the dam increases as this process continues. The major advantages of the upstream method are the cost and its simplicity. Perimeter dykes can be constructed with minimal volumes of placed fill hence decreasing the cost of the construction. However, the application of the method has some disadvantages such as it requires peripheral discharge and the beach must be wellcontrolled. It is also not suitable for significant water storage and its resistance to seismicity is poor (Vick 1983). 


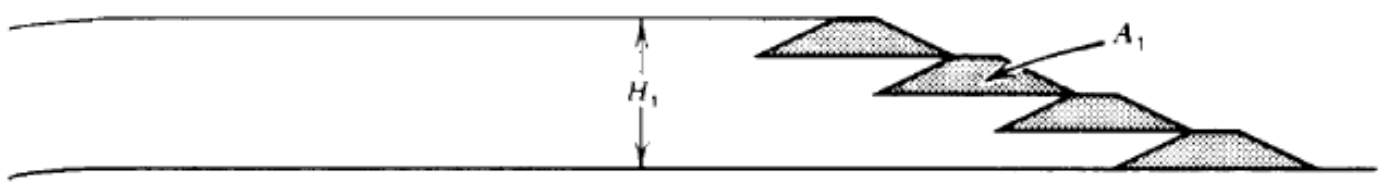

(a)

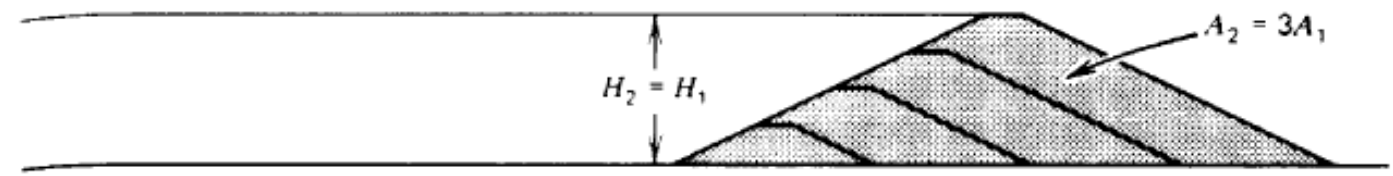

(b)

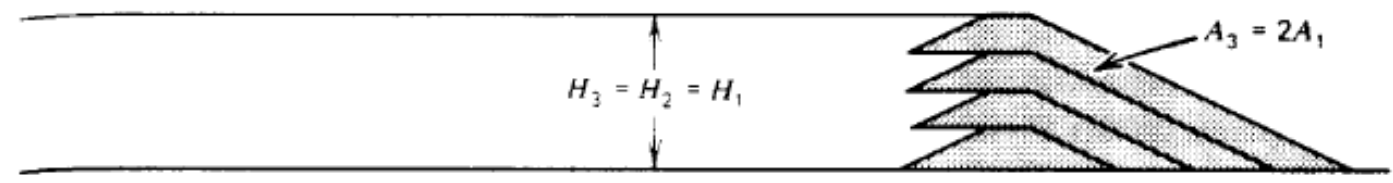

(c)

Figure 2.1: Embankment types (a) Upstream. (b) Down-stream or water-retention (c) Centerline (Vick 1983)

In the downstream method, the tailings are initially discharged behind a starter dyke. Subsequent raises are constructed on the down slope of the existing dam when required. This method is applicable to any type of tailings and the water storage capacity is high. It is more stable compared to the upstream method; however, it requires the most amount of fill material for the construction of the dam hence the relative cost of the embankment is high (Vick 1983).

The final raised embankment method is the centerline method and it is a compromise between the downstream and upstream method. Initially, a started dyke is constructed similar to the other two methods and the tailings are discharged to the dam to form a beach. The subsequent raises are constructed on the beach and on top of the downstream slope of the previous raise. It is more applicable to tailings containing sands or lowplasticity slimes. Water storage capacity is poor therefore it is not recommended for 
permanent storage. The cost of the embankment is moderate and similar to downstream method; it is more stable compared to the upstream method (Vick 1983).

Slurry deposition may have its advantages but there are also some disadvantages associated with the method. The major disadvantage is the possibility of failure of the tailing dam with the catastrophic environmental damages. According to the bulletin published by International Commission on Large Dams (ICOLD), total of 193 cases of dam failures occurred since 1960's. The main causes related to these failures are the lack of control of the water balance and the construction and general lack of understanding the control safe operations (Azam and Li 2010). One of the most common examples of catastrophic dam failure is the "Los Frailes" tailings dam failure in Aznalcollar, Spain shown in Figure 2.2. Approximately 4-5 million cubic meters of toxic tailings slurries covered thousand hectares of farmland and released acid water. It is the relative wet state of the tailing that allow for significant run out distances. That is the key danger posed by constructing slurry impoundments.

During the extraction process, a large amount of water is used. In conventional deposition, the tailings are not dewatered prior to discharge therefore loss of water to the tailing embankment is significant. In certain climates, consolidation water from the ponds surface may be collected and recovered; however, it will require treatment before it is reused in the mining operation. Conventional deposition techniques are still considered as the cheapest alternative. On the other hand, the frequency of new systems involving dewatered tailings is increasing. 


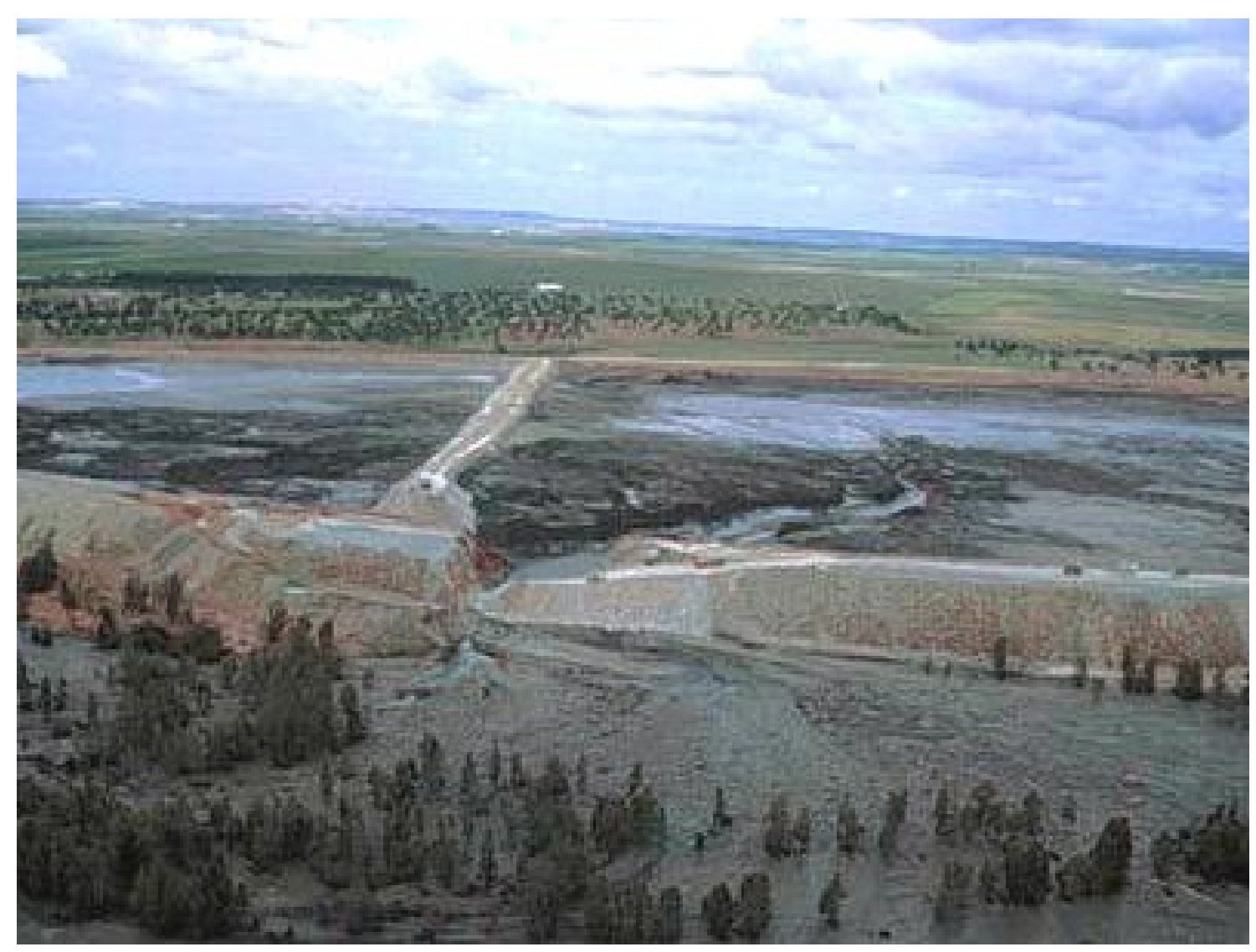

Figure 2.2: Los Frailes Dam failure (Morin and Hutt 2004)

\subsection{High-density (HD) Tailings}

High-density tailings can be defined as the tailings dewatered to a point where they will form a homogenous non-segregating mass which have sufficient strength to form gently sloped self-supporting stacks when deposited. At the end of the extraction process, the remaining material has high moisture content. The process of generating high density tailings consists of the removal of the process water before they transported to the tailings storage facility (TSF). Sufficient amount of water must be recovered to form more solid like material than a slurry one. Even though, HD tailings are almost completely saturated, the soil content on a weight percent basis is increased (Jewell and Fourie 2006). 
To produce HD tailings, sedimentation unit operations (clarifying or thickening) are most commonly used. Thickening has three main stages; first stage is the free settling where the particles are apart from each other to settle freely. The second step is hindered settling, where the particles settle as a mass at rates constrained by solid concentration, flocculation and particle density. The last stage of the process is the compression zone where the settling rate is controlled by the compressive head of all particles and the liquor above as well as the mechanical support from particles below. For the final fluid-liquid separation in the underflow from the thickeners, filters may utilize to produce paste or cake. Filtration is not cost-effective if used alone but with the thickening process, it generates the driest cake by removing as much as liquor possible (Jewell and Fourie 2006).

Ideally for high-density tailings grain size segregation does not occur during transportation and deposition. To avoid the segregation during transportation, the solid concentration per volume (Cs) must be higher than 45\% (Engman, et al. 2004). In addition, the diameter of $90 \%$ of the particles should be less than 80 microns. For paste tailings, which may be transported as a laminar flow at least $15 \%$ of the particle should have diameters less than 20 microns (Cincilla, et al. 1997).

The first attempt to produce HD tailings was under the guidance of Dr. E. Robinsky in 1973 at Kid Creek Mine in Canada. However, the original idea was fully achieved in 1995, when the technology developed and thickeners were upgraded (Jewell and Fourie 2006). Currently, more and more operations are implementing the HD tailings technology because this new technology has various advantages compared to the conventional disposal system. The cost of the initial earthwork can be significantly reduced since it 
does not require dam construction; also smaller footprint may be achieved. The risk of potential dam failures is eliminated and the stability of the tailings is improved. Another advantage is the water conservation, where considerable amount of water is recovered during thickening process and it can be recycled back to the plant process for use. This also has a positive impact on the operating costs where the water is expensive (Simms, et al. 2007).

On the other hand, there some potential disadvantages to be considered. The initial cost for the equipment is higher because of the capital cost of thickening equipment, positive displacement pumps and higher-pressure rating pipelines even though savings are achieved by water recovery. Another possible disadvantage is the defining final footprint. Since the confining perimeter embankments are not constructed, the footprint is defined beforehand. The uncertainty arises because the prediction of the beach slope angle is difficult. As a result, if the footprint is higher rehabilitation costs will increase, or unplanned dam raises may be required (Jewell and Fourie 2006). The slope also affects water balance and managing the runoff from the sloped tailings impoundments is an important aspect of their management.

\subsection{Out of pipe behavior of tailings}

A variety of theories has been investigated in the literature for the prediction of equilibrium beach profiles which will be discussed in Section 2.7 in details. During and before the deposition tailings may demonstrate various flow behaviours such as; laminar or turbulent, channelized or spreading, supercritical or subcritical. Supercritical flow may occur when they exited the pipe, leading to a hydraulic jump close to the deposition point 


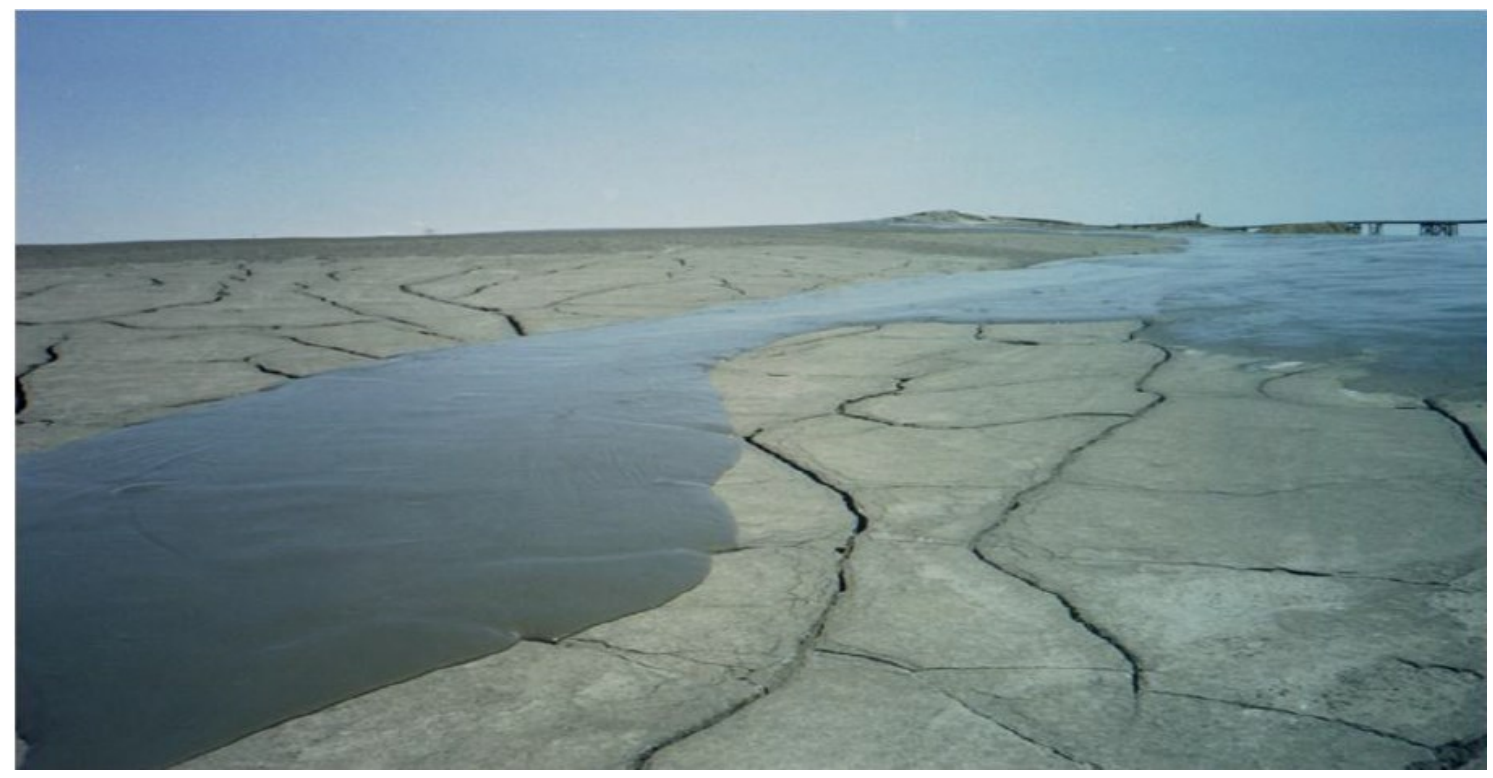

Figure 2.3: Thickened tailings running of the stack initially as the channeling flow (Mizani, et al. 2013).

and the flow may spread in subcritical state on the surface. Later on the same stack, hydraulic jump may no longer occur and the tailings first channelize and later convert to spreading flow near the bottom of the stack. Tailings no longer go through hydraulic jump but rather channelize and then developed to spreading flow state near the bottom of the stack (Mizani, et al. 2013).

\subsection{Rheology}

Rheology can be defined as the deformation and the flow of the matter (Jewell and Fourie 2006). All materials possess rheological properties which can affect their flow behaviour. In terms of fluid flow, they can be classified as Newtonian or non-Newtonian fluids. Viscosity and the yield stress are two important rheological properties which has significant effect. 


\subsubsection{Viscosity}

The flow characteristics of a fluid depend on the viscosity and it is a measure of resistance of a fluid to deform under the shear stresses. It defines the fluid's internal resistance to flow. Viscosity is a material property which relates the shear stress to the rate of shear strain. For Newtonian fluids viscosity is independent of both of these parameters.

$$
\mu=\frac{\tau}{\ddot{\Upsilon}}
$$

Dynamic viscosity, $\mu$, is used to relate the shear stress $(\tau)$ and the rate of shear srate $(\because$ and it is a constant, scalar parameter expressed in Pascal-Second (Pa.s). Shear rate has a unit of reciprocal seconds $\left(\mathrm{s}^{-1}\right)$ and defined as the change in velocity (v) divided by the distance $\left(\mathrm{h}_{\mathrm{p}}\right)$ between two points. Shear stress is the force unit per area $(F / A)$ and has a unit of Pascal $(\mathrm{Pa})$.

$$
\text { Shear rate }=\ddot{\Upsilon}=\frac{v}{h_{p}}
$$

\subsubsection{Yield Stress}

Viscoplastic materials are type of fluids which can strain only if the stress intensity exceeds a minimum value, also known as yield stress. Many industrial processes are modelled as viscoplastic materials such as slurries, cement, mud, waxy crude oils foams including concentrated mineral tailings. The behaviour of the flow can be divided into two regions; first one is the region where the stress exceeds the yield stress (yielded region) and the second region is where it is not (unyielded region). For low stress values, 
the material behaves as a solid. Above the yield stress values, the material behaves like a high viscosity fluid, allows the material to flow.

Some portions of the flow behave like a solid while the others flow since the stress is not constant in the body. The yield stress behaviour of the non-Newtonian fluids is shown below.

$$
\begin{gathered}
\ddot{\Upsilon}=0 \quad \tau<\tau_{y} \\
\tau=\tau y+\eta_{(\ddot{\Upsilon})} \ddot{\Upsilon} \quad \tau>\tau_{y}
\end{gathered}
$$

where $\tau$ is the shear stress, $\tau_{\mathrm{y}}$ is the yield stress and $\eta_{(\ddot{\Upsilon})}$ is the plastic viscosity.

HD tailings for surface deposition have yield stresses commonly below $100 \mathrm{~Pa}$. For mine back fill which is a true paste material, the yield stress values can be as high as 800 $\mathrm{Pa}$ (Jewell and Fourie 2006). For comparison, yoghurt and peanut butter have yield stresses of 50 and $500 \mathrm{~Pa}$ respectively.

\subsubsection{Slump Test}

The measurement of yield stress may be performed using a number of rheological tests, some using controlled strain, some using controlled stress, and for variety of fixtures. A vane shear fixture is very commonly used for all types of yield stress materials and allows direct and accurate determination of the yield stress value from a single point measurement (Dzuy and Boger 1985; Dzuy and Boger 1983).

The slump test has been used to estimate the yield stress. It was originally used to determine the empirical flow characteristics of fresh concrete (Jewell and Fourie 2006). The slump test is conducted using a simple slump cone or a cylinder and a ruler; therefore it eliminates the need for sophisticated equipment and allowing easy, on-site yield stress 
measurements. A detailed explanation of the slump test will be described in Section 3.2.1.

\subsection{Complications of Non-Newtonian Stress-Strain Relation}

General characterization of Non- Newtonian flows can be divided into four main categories; flows dominated by shear viscosity, slightly elastic flows, nearly viscometric flows, and complex flows involving highly elastic liquids (Crochet, et al. 1984). High density tailings fall into the first category.

Normal stress differences are low with respect to shear stresses for flows dominated by shear viscosity, and therefore elasticity properties of the fluid do not have a major effect on the geometry of the flow.

A variety of constitutive equations of various forms and complexity have been proposed in the literature for the modeling the rheological behaviour of viscoplastic material (Balhoff, et al. 2012; Bird, et al. 2007; Shook and Roco 1991; Spelay 2007). The simplest and the most relevant yield stress model is the Bingham fluid. For the shear thinning property of the plastic viscosity Herschel-Buckley model is preferred. Another simple two-parameter model for shear thinning property is Casson which is originally developed for the viscous behaviour of blood. Compared to Herschel -Buckley model, the total plastic viscosity depends on the yield stress in Casson model. Constitutive relations for different type of models are presented below. 
Newtonian Fluid

Power Law Fluid

Bingham Fluid

Herschel-Bulkley Model

Casson Model

$$
\begin{gathered}
\tau=\mu \ddot{\Upsilon} \\
\tau=K \ddot{\Upsilon}^{n} \\
\tau=\tau_{y}+\mu_{p} \ddot{\Upsilon} \\
\tau=\tau_{y}+K \ddot{\Upsilon}^{n} \\
\tau^{0.5}=\tau_{c}^{0.5}+\left(\mu_{c} \ddot{\Upsilon}\right)^{0.5}
\end{gathered}
$$

where $\tau$ is the shear stress, $\mathrm{K}$ is the Power-law and Herschel-Buckley model consistency index, $\mu$ is the dynamic viscosity and $\ddot{\Upsilon}$ is the rate of shear.

\subsection{Post-deposition Behavior of Tailings}

Deposition of thickened or high-density tailings is covered in the previous section but it is also important to understand the post-deposition behaviour of the material and how it might influence stack geometry. After discharge the tailings in the storage area will form sloping conical stacks. The deposited layers will gain strength through selfweight consolidation and desiccation before the next layer is deposited. The underlying tailings, if they remain sufficiently soft, may fail during placement of the new tailings and these failures will affect stack geometry.

It is a known fact that the volume will be reduced due to settling and desiccation. In order to estimate the performance of the stack, there are some hydrological processes must be taken into consideration such as evaporation, precipitation, infiltration and runoff. The volume of the tailings will decrease as the dissipation of excess pore-water pressures occur during the settlement. Negative higher pore water pressures also contribute to the volume decrease which results in shrinkage of the material. Therefore, tailings with higher void ratios will take longer time to consolidate (Fisseha, et al. 2010). 
The most important factor that promotes volume change is the evaporation because it supports desiccation with increased density and gained strength; as a result steeper deposition angles can be achieved in the field. Evaporation driven desiccation and subsequent desaturation of the thickened gold tailings has been studied before (Fisseha, et al. 2010; Simms, et al. 2010; Simms, et al. 2007). High evaporation rates in arid climates leads to desiccation and desaturation results reduction of moisture content and compressibility of the material.

There are three main stages of evaporation and in the first stage, the actual evaporation from saturated or near-saturated soil proceeds at the maximum, or potential, the rate of total suction values are less than about $3000 \mathrm{kPa}$ at the soil surface. Also, the rate of evaporation is only a function of climatic conditions and water transported within the soil as liquid. Total suction of the soil is functionally related to relative humidity $(\mathrm{RH})$ of the soil pore air. For the suction values greater than $3000 \mathrm{kPa}$, the relative humidity starts to decline as a result vapour pressure begins to decline as well. It will cause a reduction in vapour pressure gradient and it lowers the rate of evaporation because the evaporation is strongly controlled by the difference between vapour pressure of pore air at the soil surface and the vapour pressure of air below the soil. This declining stage of evaporation is known as Stage II evaporation.

The water transport in the second stage is a combination of liquid flow and water vapour diffusion. The total suction at the soil surface may continue to increase until a new equilibrium stage is achieved in the third stage of evaporation. At the last stage of evaporation, relative evaporation will have a low but constant rate of evaporation and the 
supply of moisture to the surface of the soil is largely due to water vapour transport by diffusion.

Excess evaporation can form cracks on the surface and allows for ingress of oxygen which can lead to acid generation. Evaporation from saline water surface is always less than fresh water surface due to decline in number of water molecules per unit volume. Also, thermal energy of water molecules is affected by molecular interaction due to the presence of salt. Beneath the salt-encrusted surface, salt concentration in soil pore water is approximately equal to saturation condition. That can reduce the equilibrium vapour density by $76 \%$ in comparison to equivalent freshwater (Fujiyasu and Fahey 2000). As desaturation continues, salt accumulation to the surface occurs for saline tailings which is more common in semi-dry and dry areas where the ore is processed with extremely saline ground water. Cracking may increase the evaporation rate while salt accumulation on the surface suppresses it (Fisseha, et al. 2010; Simms, et al. 2007).

The rate of drying can be predicted by knowing some hydro geotechnical properties of the materials such as soil-water characteristics, saturated hydraulic conductivity, the potential rate of evaporation, with use of unsaturated flow models coupled with atmospheric boundary conditions (Fisseha et al. 2010).

\subsection{Prediction of Deposition Geometry}

Predicting the stack geometry and the overall slope angle is critical to stack design because it determines the footprint, storage capacity, susceptibility to erosion and slope stability. For instance, if the slope is too small it increases the footprint required for 
deposition or if the angle is too steep, it promotes erosion and decreases the geotechnical stability of the stack.

A variety of theories have been investigated in the literature for the prediction of equilibrium beach profiles. Fitton's beach slope method and McPhail's stream power method are based on the analysis of channelized flow developed during the deposition. Lubrication Theory investigates the prediction of equilibrium profiles for spreading tailings flow (Simms, et al. 2011). They are the most commonly used methods to estimate beach slope.

\subsubsection{Fitton's Beach Slope Method}

Fitton's beach slope method suggests that beach slope is constrained by the selferoding behaviour of tailings as they flow in channel. The depth and the shape of the channel must be known in order to calculate the hydraulic radius and average velocity. An empirical relationship between the depth and the flow rate under equilibrium conditions (turbulence flow conditions hence non-segregating) is shown below.

$$
d=12.2 Q^{0.6}
$$

where $\mathrm{d}$ is the depth of flow in millimetres, $\mathrm{Q}$ is the flow rate in litres per second (Fitton, et al. 2006). The channel is assumed to be semi-circular in cross section.

This relationship is then used to calculating the channel bed angle $(i)$ with the use of critical velocity $(V)$.

$$
i=\frac{100 \tan \arcsin 0.073 V^{2}}{\left(\frac{8 \rho V^{2}}{\tau_{y}+K\left(2 v / R_{H}\right)}\right)^{0.25} 2 R_{H} g}
$$


where $\mathrm{v}$ is the average velocity in the channel below which sedimentation occurs, $\tau_{\mathrm{y}}$ is the yields tress of the slurry, $\mathrm{R}_{\mathrm{H}}$ is the hydraulic radius, $\mathrm{K}$ is the fluid consistency index from Herschel and Bulkley model and $\mathrm{i}$ is the channel bed angle equivalent to the percentage (Fitton, et al. 2006). The critical velocity ( $V$ ) is estimated by an empirical function that depends in grain size.

\subsubsection{McPhail's Stream Power Method}

McPhail's stream power method relates the beach slope profile to the energy dissipation of energy of the tailings as they flow in the channel. Stream power is calculated from the density $(\rho)$, flow rate $(\mathrm{Q})$, gravitational acceleration $(\mathrm{g})$ and the energy head (McPhail, et al. 2008).

$$
P_{s}=\rho g Q H_{e}
$$

The velocity head $\left(\mathrm{v}_{x}\right)$ can be calculated using the energy head at any point along the beach. Hence the relation of the stream power and the velocity head at any point at a distance of $\mathrm{x}$ from the start of the beach can be defined as;

$$
\mathrm{P}_{s_{x}}=\rho Q g\left(\frac{v_{x}^{2}}{2 g}\right)
$$

The entropy maximisation approach could be used to estimate the stream power at any point along the beach. The essence of this method is the infinite number of curved could define the change in the stream power along the beach. The formulation of the power stream method using entropy maximization is defined below.

$$
\mathrm{P}_{s}(\mathrm{x})=-\frac{1}{\beta} \ln \left[\left(1-\exp ^{-\beta P_{0}}\right) \frac{x}{L}+\exp ^{-\beta P_{0}}\right]
$$


Where $\mathrm{P}_{\mathrm{o}}$ is the stream power at the start of the beach and $\mathrm{L}$ is the distance from the start of the beach to the edge of the supernatant pond. The constant $\beta$ is a parameter emerging from the mathematical derivation of Eq. (2.12). The slope of the stream power curve $\left(\mathrm{S}_{\mathrm{B}}\right)$ is then obtained by differentiating Eq. (2.12) with respect to $\mathrm{x}$ as follows;

$$
S_{B}=-\frac{\left(1-\exp ^{\beta P o}\right)}{\left(L \beta \exp ^{\beta P o}\right)}
$$

Finally the elevation, $y$, of the beach at any point can be determined by using the following formula and the small increment of $\Delta \mathrm{x}$ from the start of the beach (McPhail, et al. 2008).

$$
y=y(x+\Delta x)+S_{0}(x) \Delta x
$$

\subsubsection{Lubrication Theory}

Lubrication Theory estimates the equilibrium geometry of the stack by simplifying Navier-Stokes momentum and continuity equations to relatively simple equations. Several assumptions are used to simplify the equations such as; the fluid is homogeneous, the ratio of thickness to length is small and the fluid has a small velocity hence the ratio of inertia to viscous force vanishes from the equations. These equations are later solved analytically for yield stress fluids under special geometries and conditions. Balmforth, et al. (2002), Liu and Mei (1989), Yuhi and Mei (2004) used the Lubrication Theory applications to viscoplastic flows such as mud and lava flows. The balance of momentum equation is presented as; 


$$
\rho g \sin \theta-\frac{\partial p}{\partial x}+\frac{\partial \tau}{\partial z}=0
$$

where $\rho$ is density, $\mathrm{p}$ is pressure, $\tau$ is shear stress, $\mathrm{g}$ is gravitational acceleration and $\theta$ is the angle of the inclined surface. The pressure distribution is assumed to be hydrostatic:

$$
P=\rho g(h-z) \cos \theta
$$

where $h$ is the depth of the free surface of the flow. Then differentiating Equation 2.17 to substitute it into the left hand side of the Equation 2.16; shear stress equation in terms of depth $\mathrm{z}$ is obtained.

$$
\tau=\rho g(h-z) \cos \theta\left(\tan \theta-\frac{\partial h}{\partial x}\right)
$$

By setting $z=0$, the expression for the steady-state profile of a Bingham fluid can be obtained. The equation for the flat bed is defined below;

$$
\tau_{y}\left(x-x_{0}\right)=\frac{\rho g}{2}\left(h^{2}-h_{0}^{2}\right)
$$

where $\mathrm{h}_{0}$ is the height at $\mathrm{x}_{0}$.

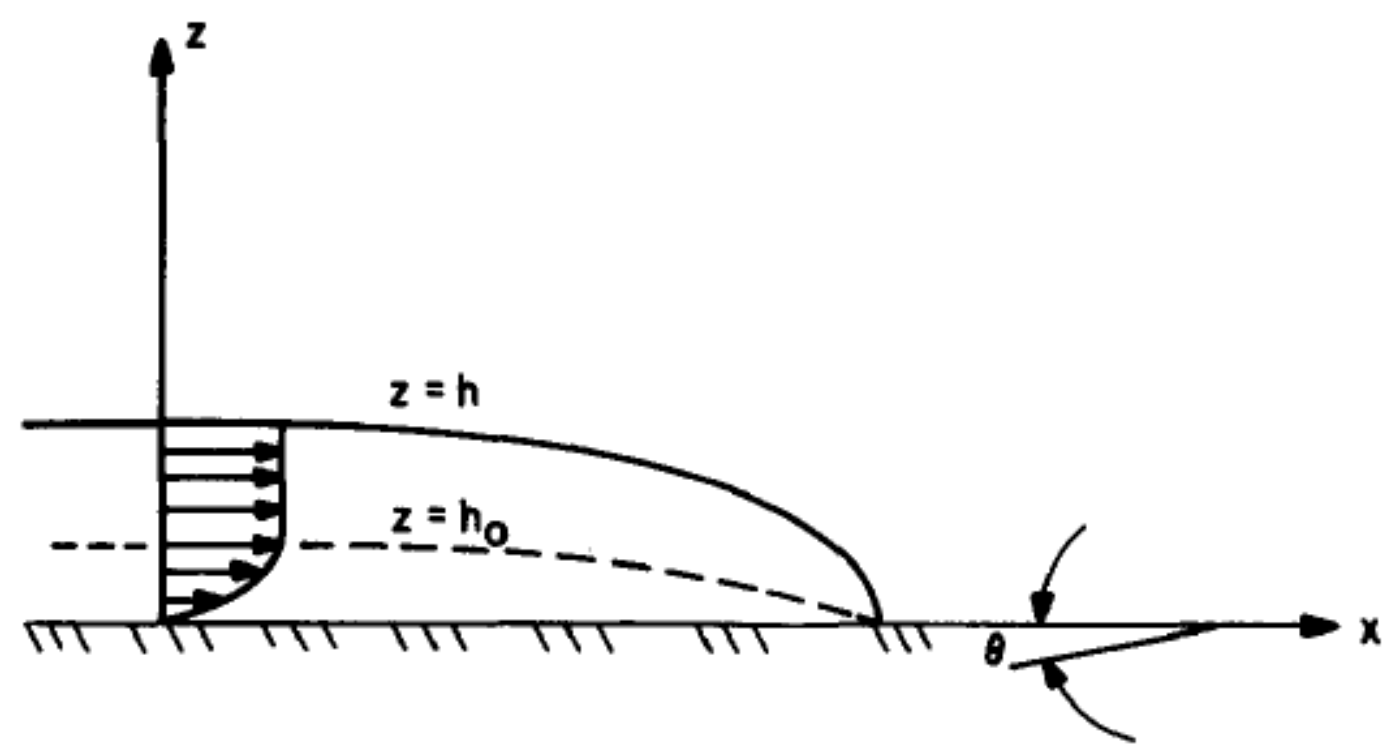


Figure 2.4: The definition sketch (Liu \& Mei, 1990)

The equations for two and three dimensional flow of a slow spreading fluid mud over a conical surface is described in Yuhi and Mei (2004).

$$
h^{\prime}-h_{0}{ }^{\prime}+\ln \left(1-h^{\prime}\right)=x^{\prime}-x_{0}{ }^{\prime}
$$

where

$$
\begin{gathered}
h=h^{\prime}\left(\frac{\tau_{y}}{\rho g \sin \theta}\right) \\
x=x^{\prime} \cot \theta\left(\frac{\tau_{y}}{\rho g \sin \theta}\right)
\end{gathered}
$$

Simms, et al. (2007), Henriquez and Simms (2009) and Mizani, et al. (2013) studied the applicability of the theory for small scale laboratory tests for kaolinite and gold tailings. Henriquez and Simms (2009) found that the theory is applicable to describe the HD tailings flow and showed that the overall deposition angle is scale dependent for single layer deposits. Also, Mizani et al. (2013) applied the Lubrication Theory to multi layer depositions and found that the first few layers can be well predicted but the geometry start to exhibit a convex profile as the number of layers deposited increase. By contrast, LT predicts an increasing linear slope as the number of layers increases.

\subsection{Numerical Modeling of Non-Newtonian fluids}

Three of the most popular numerical methods for analyzing non-Newtonian fluid problems are reviewed in the following sections: Finite volume, finite element and Smoothed Particle Hydrodynamics (SPH). 


\subsubsection{Finite Volume Method}

Finite volume method involves integrating partial differential equations over control volumes of mesh with respect to time and space (Spelay 2007). The domain is divided into small control volumes and the constitutive equations are integrated over each control volume. The main advantage of the method is to conserve quantities such as mass, energy and momentum. Several researches investigated the flow behaviour of non-Newtonian fluids using finite volume method. Spelay (2007) focused on the use of two-layer macroscopic model (involving less concentrated top layer with higher velocity and more concentrated, slower bottom layer) for the modelling of non- Newtonian viscoplastic flow in a pipe.

Ghosh and Shook (1989) investigated the applicability of two-layer model for power law fluids and it was found that the model was useful for the low velocity conditions however; it predicts negative velocities in the lower layer suggesting an incorrect interfacial friction factor. Hill (1998) and Hill and Shook (1998)Hill and Shook (1998) tested the same model in laminar and turbulent flow to understand the behaviour of coarse solid transport in Bingham carrier fluid. They have found that, two-layer model is useful for laminar flow modelling. The model produced satisfactory results in turbulent flow but lower layer concentration components still need more investigation.

Another example of finite volume method applied to viscoplastic fluids is reviewed in Keslerová and Kozel (2010). They focused on the numerical modelling of Newtonian and non-Newtonian fluids (which is defined as a power-law fluid) through a branching channel (one entrance and two exits) using finite volume method solving Navier-Stokes 
equations. Several power-law index values are tested and the convergence history proved the robustness of the method.

\subsubsection{Finite Element Method}

Finite element method divides the domain into smaller interconnected sub domains called finite elements. The governing equations are solved in each element eventually calculating the final solution. The main difference of this method from previous one is that the equations are multiplied with a weight function before integrated over the domain. This method is used for many engineering applications; mainly in the field of structural engineering, heat transfer problems, fluid dynamics, seepage flow and electrical or magnetic flows.

Dean, et al. (2007) and Glowinski and Wachs (2011) investigated the Bingham flow in cylinder using finite element method. The results showed that the main difficulty in the solution of viscoplastic flows problems is tracking the non-differentiability of the constitutive law at the yield point, in other words tracking the yield surfaces. This is similar to the problem of modelling advective flow using differential equation based models.

Naef, et al. (2006) focused on the development of a new model (DFEM-1D) to simulate the laminar debris flows. This new model is based on a finite element solution of depth averaged shallow water equations. DFEM-1D uses the detailed topography and variety of flows, from granular to viscous, are simulated using it. They focused on the one-phase models which describes the flow behaviour and only applicable to laminar flows. It was found that for granular debris flows, a turbulent flow resistance term is 
essential in the models since Bingham or other approaches under predict observed front velocities. The model also under predicts the flow depth or discharge if the model is calibrated using run out and velocity data. It was found that the contribution of yield stress is only valuable at the final stopping regime. Importance of the hydrograph (using various shapes of inputs) is also studied and it was found that it affects the peak charge about the first one kilometer and the small changes over the downstream.

Bovet, et al. (2010) studied the numerical modelling of snow avalanche dynamics using finite element method. The model they focused on is the simulation of the movement of an avalanche on an inclined slope including a snow cover (defining the material as a shear thinning fluid. The simulations are carried using COMSOL Multiphysics. Introduction of frictions forces are introduced in the model to stop the avalanches. The simulations are compared to the experimental data for the velocity profile. The results demonstrated that the estimation of the upper part of the flow gives good correlations. However, due to large meshing, the velocity at shear boundary layers was not fitted. Finally, the agreement between the experimental data of the front velocity with the simulated data is satisfactory. The model needs to be improved because it does not take into account of confinement and the expansion of the flow due to the real topography.

\subsubsection{Smoothed Particle Hydrodynamics (SPH)}

SPH is a mesh free, Lagrangian particle method for modeling fluid problems. The flow is replaced by a set of particles and the equations of motion on each particle are integrated by the method. SPH interpolates the physical quantities of neighbouring 
particles to obtain the properties of each particle and the fluid moved according to those calculated values. (Monaghan 2012; Monaghan 2005). The SPH method has been applied to various problems such as dam breaks and plunging waves for the weakly compressible fluids, flow of two fluids with different densities, landslides, the fluids interacts with a moving body, non-Newtonian fluid simulations, to name just a few examples.

Zhu, et al. (2010) investigated the determination of plastic viscosity from vane rheometer measurements by applying a Lagrangian formulation of Navier-Stokes equation based on SPH approach. Vane geometries are commonly used to determine the rheological properties of non-Newtonian fluids but due to the complex flow geometry of the vane, relating the measured quantities to the rheological properties is a challenge for many different reasons such as the lack of information of the actual flow rates and slip occurring at fluid or wall boundaries. Usually the numerical simulations involving nonNewtonian fluids with complex geometries are determined by macroscopic approaches such as finite difference, finite element, finite volume or boundary element methods using Eulerian scheme.

Zhu, et al. (2010) focused on the numerical simulations based on SPH method for the flow of Bingham fluid in a vane rheometer under relatively high shear stresses which is accomplished to validate the applicability of the method to determine the rheological properties using vane rheometer. The continuum and momentum equations in SPH form are the governing equations with the addition of Bingham fluid constitutive equation added to momentum equations. For the validation of the numerical scheme, Poiseuille flow of Bingham fluids and the tangential angular flow of Newtonian fluids are 
simulated. The velocity profiles of Poiseuille flows with different Bingham numbers under various applied angular viscosities are investigated. It was found that the Bingham number plays an important role in determining the torques transmitted to the outer wall of the vane rheometer; as the Bingham number increases, un-yielded material in the inner blade region increases and the flow conditions becomes similar to the conditions in coaxial cylinder rheometer. Also, a numerical simulation of the tangential flow of an incompressible Newtonian flow is performed in order to determine the velocity and stress distributions between two coaxial cylinders (the outer cylinder is rotated while the inner cylinder kept fixed). It was proved that the computed velocities are very similar to theoretical results and the maximum relative errors are very small hence the results for shear stress are in good agreement with the analytical values. However, they also found that the stress values are declined slightly when the particles are close to the inner wall.

Hosseini, et al. (2007) presented a new method which is fully explicit in time and uses a three-step algorithm to solve two dimensional non-Newtonian fluid flow problems. The model is tested with three different cases involving a simulation of a free surface flow, internal flow and interface between two fluids for both Newtonian and nonNewtonian fluid in order to prove the applicability of the SPH algorithm developed.

For the first test case, the simulation is similar to broken dam analysis studied in Shao and Lo (2003) both for Newtonian and non-Newtonian fluids. The first test case consists of a rectangular column of fluid at a slope, kept between a fixed wall and a temporary wall, which is later removed and the fluid collapse under the influence of gravity. The proposed method gives similar results to the experimental data obtained from Komatina and Jovanovic (1997). 
In the second test case, a tangential creeping flow in a viscometer which is made of two coaxial cylinders is investigated. The inner cylinder rotates while the outer cylinder is kept fixed with a constant angular velocity. Since the boundary has a circular shape, filling the domain with square particles to create sharp edges and the particles have less neighbouring particles which leads to increasing numerical errors. This can be prevented by changing the initial spacing between the particles in certain regions.

The final test case investigates the laminar gravity current of fluid mud on an inclined bed. The problem is solved by using Bingham plastic rheological model and the sediment settling and interfacial shear stress between the mud layer and the overlying water is neglected. It was found that the simulation is in a good agreement with the computed tangential velocity along the slope bed. The progress of the mud flow is presented in the following figure at different times (Hosseini, et al. 2007).
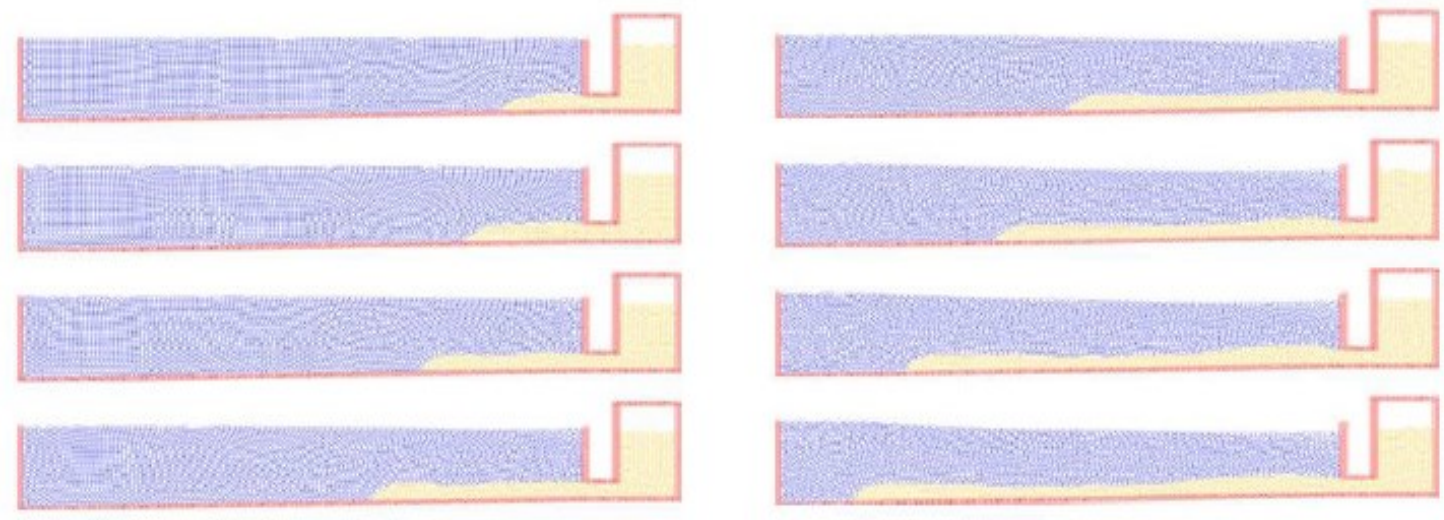

Figure 2.4: Mud front at different times ( $\mathrm{t}=12,16,20,24,28,32,36$ and $38 \mathrm{~s}$ (Hosseini, et al. 2007).

Mudflows and debris flows are gravity-driven unsteady flows with a high concentration of soil and water mixture with large grain size distribution. In order to reduce the damages caused by mudflows, many structures are installed and they are 
submitted to normal impacts which are designed to reduce the flow dynamics and the subsequent effects. Laigle et al. (2007) focused on the effect of these protection structures on mudflows; such as momentum reduction in the mudflow as well as the resistance and the impact of force on the structures. Flow structure interaction theory is based on 2D vertical model developed by LaChamp (2003) using SPH numerical method. HerschelBuckley model is adapted for the mudflow and the rheology is determined by using local viscosity within the SPH approach. Laigle, et al. (2007) first conducted a simulation of unsteady free-surface flows of viscoplastic fluid in the absence of the structure. Based on these simulation two main topics are investigated; flow downstream of the obstacle to determine the momentum reduction resulting in the mudflow when the structure is introduced in the problem and the pressure in the local area of the upstream face of the structure in order to determine the stress applied on this structure. The results obtained are similar to theoretical results.

Capone, et al. (2010) simulated the submarine landslides as a non-Newtonian fluid while investigating the water wave generated given by the impact between the landslide and the water. The model consists of a mass of sand slide from a slope of $45^{\circ}$. In order to determine the suitability of the model to simulate deformable landslides and its interaction with water, the physical experiment results are compared with the numerical outcomes. The rheological model used is known as the "bi-viscosity" model which is also chosen for this research. The detailed explanation of the model is presented in Chapter 5. The model tested gave excellent agreement with the experimental results with the use of simple and versatile rheological model. 


\subsection{The SPH Method}

SPH is a mesh free, Lagrangian particle method where the fluid is replaced by a set of particles to acquire approximate numerical solutions. SPH integrates equations of motion on each particle to obtain its individual material properties. The relevant physical quantities are determined by interpolating the quantities of the neighbouring particles and the fluid is moves accordingly. (Monaghan 2012; Monaghan 2005).

The Lagrangian nature of SPH has some advantages when compared to the other Eulerian computational methods. For example, computational effort is mainly concentrated on the areas where the fluid is present hence time is not wasted calculating empty areas. SPH does not require the use of a grid, and finding the free surface is trivial. There are no constraints on the geometry or the initial conditions since they can be programmed without the need of complicated gridding algorithms used in finite element or finite volume methods. However, there are also some limitations of the method. The boundary conditions are hard to implement and the penetration of the fluid particles into the boundaries must be avoided. The interpolation method used in SPH is very simple and it can be affected by the particle disorder (large gaps between the particles). Particle disorder will happen during the simulation of SPH problem; however the results must be compared with the experiments or known values in order to determine the accuracy of the SPH model. Finally, the computational speed is slower when compared with other gridbased methods because the time steps are related to the speed of sound; for higher speed of sound values smaller time steps are required. 


\subsubsection{General Formulation of SPH}

A set of particles such that the particle $\mathrm{b}$ has a mass $m_{b}$, density $\rho_{b}$ and position $r_{b}$. The main principal in SPH is to approximate any function A(r) (such as pressure and viscosity term) by the following form;

$$
A(r)=\int A\left(r^{\prime}\right) W\left(r-r^{\prime}, \mathrm{h}\right) d r^{\prime}
$$

where $d r^{\prime}$ is the volume element, $h$ is the smoothing length and $W\left(r-r^{\prime}, h\right)$ is the weighting function or kernel. The approximation above will lead to approximation defined in Equation 2.24 where the summations are over all the particles. This is the general formulation in SPH; for example the density can be calculated by replacing quantity $A$ by $\rho_{a}$ and $r$ by $r_{a}$ (Eq. 2.28) (Gómez-Gesteira, et al. 2010);

$$
A(r)=\sum_{b} m_{b} \frac{A_{b}}{\rho_{b}} W_{a b}
$$

For a numerical method to converge, it must have a certain level of consistency which is measured by the degree of polynomial of the kernel function can reproduce. The weighting function or the smoothing kernel is critical for the performance of an SPH model. The weighing functions make sure that the linear functions are perfectly interpolated and their gradients are obtained. General formula of the kernel is given as;

$$
\begin{aligned}
& \int_{\Omega} W\left(r-r^{\prime}, h\right) d r^{\prime}=1 \\
& \sum_{b=1}^{N} W\left(r-r_{b}, h\right) \Delta r_{b}=1
\end{aligned}
$$

Various forms of kernels such as Gaussian, polynomial and cosine kernels are proposed in the literature. Kernel function is used to reduce the error which is developed 
by the interpolation function. One of the most popular kernel function based on cubic spline function is defined as below.

$$
W(r, h)=\alpha_{D}\left\{\begin{array}{ll}
1-\frac{3}{2} q^{2}+\frac{3}{4} q^{3} & 0 \leq q \leq 1 \\
\frac{1}{4}(2-q)^{3} & 1 \leq q \leq 2 \\
0 & q \geq 2
\end{array}\right\}
$$

where $q$ is the non-dimensional distance between particles $(r / h), r$ being the distance between particles $a$ and $b, h$ is the smoothing length. $h$ is also known as the influence domain since it controls the size of the area around particle a where the contribution of the neighbouring particles cannot be neglected. Finally, $\alpha_{D}$ is a normalization constant defines as $10 /\left(7 \pi \mathrm{h}^{2}\right)$ and $\left.1 / \pi \mathrm{h}^{3}\right)$ for $2 \mathrm{D}$ and 3D respectively (Gómez-Gesteira, et al. 2010; Hosseini, et al. 2007).

The use of cubic spline kernel leads to high computational efficiency and its interactions are exactly zero for q values larger than two. The first and second iterative of this kernel is continuous hence the interpolation is not sensitive to particle disorder. Higher-order splines may be used but that will lead to interaction with more particles thus it requires more computational time (Hosseini, et al. 2007). Defining the fluid as a weakly compressible fluid rather than incompressible fluid will decrease the computational time since solving the Equation of State is faster than the solving Poisson's Equation.

The governing equations are allowing three laws of conservations; mass, momentum and energy. Continuity equation relates the mass to density approximation presented below. 


$$
\begin{gathered}
\rho_{a}=\sum_{b=1}^{b} m_{b} W_{a b} \\
\frac{d \rho_{a}}{d t}=\sum_{b} m_{b} v_{a b} \cdot \nabla_{a} W_{a b}
\end{gathered}
$$

Equation 2.28 could be possibly used to as a continuity equation; however, when modelling a free surface problem, this equation cannot be used directly since the density will decrease close to the interfaces such as fixed boundaries or free surfaces. As a result, further derivation is required which resulted as the Equation 2.29. With the usage of this approximation, the densities of all particles are defined initially and the changes in the density are related to the neighboring particles in the support domain. Conservation of mass defined above is in the form of weakly compressible flow to avoid solving Poisson' equation at each time step The conservation of momentum equation for a Newtonian fluid in a continuum field is represented below (Gómez-Gesteira, et al. 2010).

$$
\frac{d v}{d t}=-\frac{1}{\rho} \nabla P+g+\Phi
$$

where $\mathrm{g}$ is the gravitational acceleration and $\Phi$ is the diffusion term. There are three main diffusion terms used in the momentum equations; artificial viscosity, laminar viscosity and full viscosity. An artificial viscosity term was proposed by Monaghan (1992) (presented in Eq. 2.33) and it is used to stabilise the numerical algorithm. It does not have a direct relation to real viscosity but the dynamic viscosity is one of the coefficients used in the calculation. It is used very often by other researchers due to its simplicity. Thus, artificial viscosity term is also selected for the present work however slight modifications are applied which are discussed in Chapter 5 in details. Conservation of momentum equation for a Newtonian fluid is defined as; 


$$
\frac{d v_{a}}{d t}=-\sum_{b} m_{b}\left(\frac{P_{a}}{\rho_{a}^{2}}+\frac{P_{b}}{\rho_{b}^{2}}+\Pi_{a b}\right) \nabla_{a} W_{a b}+\mathrm{g}
$$

The pressure gradient term in symmetrical form is expressed in SPH notation as;

$$
\left(-\frac{1}{\rho} \nabla P\right)_{a}=-\sum_{b} m_{b}\left(\frac{P_{b}}{\rho_{b}^{2}}+\frac{P_{a}}{\rho_{a}^{2}}\right) \nabla_{a} W_{a b}
$$

$\Pi_{a b}$ is the viscosity term, defined as;

$$
\begin{gathered}
\Pi_{a b}= \begin{cases}\frac{-\alpha_{\mu} c_{a b} \mu_{a b}}{\rho_{a b}} & v_{a b} r_{a b}<0 \\
0 & v_{a b} r_{a b}>0\end{cases} \\
\text { with } \mu_{a b}=\frac{h v_{a b} r_{a b}}{r_{a b}^{2}+\eta^{2}}
\end{gathered}
$$

where $r_{a b}=r_{a}-r_{b}, v_{a b}=v_{a}-v_{b}$; being $r_{a b}$ and $v_{a b}$ corresponds to the position and velocity between particles $a$ and $b, c_{a b}$ being the average speed of sound between the particles a and $\mathrm{b}, \eta^{2}=0.01 h^{2}$ is a corrective term introduced to prevent singularity when $\vec{r}_{a b}=\boldsymbol{v}$ and $\alpha_{\mu}$ is the dynamic viscosity of the Newtonian fluid (Gómez-Gesteira, et al. 2010).

Finally, the last governing equation is the conservation of energy formulation which is based on the first law of thermodynamics where the change in the thermal energy us equal to the energy added the system minus the work conducted. Since there is no heat added to the system the only source of energy consists of the work done by the body forces in the infinitesimal fluid cell. Thermal energy associated with each particle is calculated using (Gómez-Gesteira, et al. 2010); 


$$
\frac{d e_{a}}{d t}=\frac{1}{2} \sum_{b} m_{b}\left(\frac{P_{a}}{\rho_{a}^{2}}+\frac{P_{b}}{\rho_{b}^{2}}+\psi_{a b}\right) v_{a b} \nabla_{a} W_{a b}
$$

where $\psi_{\mathrm{ab}}$ refers to the viscosity term which is calculated by using the approach above (Eq. 2.33). The total energy of the system is calculated by summing kinetic, potential and thermal energies. Finally, the particles are moved by using the XSPH variant (GómezGesteira, et al. 2010).

$$
\frac{d r_{a}}{d t}=v_{a}+\frac{1}{2} \sum_{b} \frac{m_{b}}{\frac{\rho_{a}+\rho_{b}}{2}} v_{a b} W_{a b}
$$

The formulas represented above are applicable for weakly compressible Newtonian fluids. In order them to become suitable for non-Newtonian problems some modifications have to be conducted, specifically the viscosity term. The modifications and the rest of the theory, such as time-stepping and density re-initialization, used in the model described in Chapter 5 thoroughly.

\subsubsection{Boundary Conditions}

In SPH formalism, the boundaries are represented as a set of particles and when a fluid particle reaches to a boundary, the only interaction the particle will get is from the particles located inside the system. It can create some problems because of the different nature of the variables to solve; for example the velocity of the particles can drop to zero when it is approaching a boundary while the density does not (Monaghan 2012; Hosseini et al. 2007; Shao and Lo2003). There are three main boundary conditions applied in SPH. 
a. Ghost Particles: These particles are the images of the fluid particles. A virtual ghost particle is generated outside the system when a real particle approaches a boundary. Both of these particles have the same density and pressure values but opposite velocities. The implementation of the model may become very complicated sue to the changing number of these ghost particles at each time step (Crespo 2008). This strategy is mostly used in graphical industry and it is not tested further for other methods since the appearance is more important than the accuracy. This approach doesn't require the use of artificial terms and external particles; it displays smooth behavior in modeling flows with less curved boundaries. However, it also leads to numerical errors when model of fluids with curved solid surfaces are developed (Zhu et al. 2010; Ellero et al. 2002).

b. Repulsive particles: One of the strategies for the boundary conditions in SPH simulations is the fixed boundary particles, originally developed by Monaghan (1994). These boundary particles generate central forces on the fluid particle to prevent the particles crossing a solid boundary. However, the particle densities must be evaluated near the wall since the densities of the particles approaching to the surface drops rapidly (Zhu, et al. 2010)

c. Dynamic particles: These particles have to satisfy the same equations as the fluid particles; although, their positions remains unchanged. One of the advantages of this boundary condition is that their computational simplicity; they can be calculated inside the same loops as the fluid particles therefore diminishes the computational time significantly. This boundary condition is selected for the simulations conducted in this research. 
When a fluid particle approached to a boundary particle, the density and the pressure of a boundary particle increases according to the equation 2.29. As a result, the force exerted on a fluid particle increases due to the pressure term, $\left(P / \rho^{2}\right)$ in the momentum equation (Eq. 2.31). When the distance between the boundary particle and the fluid particle is smaller than $2 h$, the force exerted on the incoming particle generates the repulsion mechanism (Gómez-Gesteira, et al. 2010).

\subsubsection{SPHYSICS}

SPHYSICS is an open source Smoothed Particle Hydrodynamics code inspired by the formulations created by Monaghan (1992). The code is developed by several researchers at the John Hopkins University (U.S.), the University of Vigo (Spain), the University of Manchester (U.K) and the University of Rome La Sapienza (Italy). the program is originally released in August 2007 and its available for public use at http://wiki.manchester.ac.uk/sphysics (Crespo 2008).

The program is written to solve free surface Newtonian fluid problems using the SPH method. It is coded entirely in FORTRAN and the post-processing is done with MatLab. The code is applicable to various types of problems such as; dam breaks, wave breaking, sliding and/or floating objects, wave impact on a structure etc. The inputs in the model are generated in the modular form and variety of features are available to chose different compiling options presented below (Crespo 2008). 


\begin{tabular}{|l|l|}
\hline Dimensions & 2D \\
& $3 \mathrm{D}$ \\
\hline Time integration scheme & Predictor-corrector \\
& Verlet \\
& Beeman \\
& Symplectic \\
\hline Kernel functions & Gaussian \\
& Quadratic \\
& Cubic Spline \\
& Wendland Quintic \\
\hline Boundary Conditions (B.C.) & Dynamic B.C. \\
& Repulsive B.C. \\
& Periodic Open Boundaries \\
\hline Viscosity Treatment & Artificial \\
& Laminar \\
\hline Sub-Particle Scale(SPS) Turbulence \\
\hline Moving Objects & Floating object \\
\hline Mensity Reinitialization & Moving Gate \\
& Wavemaker \\
\hline & Sliding Wedge \\
\hline & Shepard Filter \\
& Moving Least Square (MLS) \\
\hline & Kernel Gradient Correction \\
\hline & Window: Visual Fortran Compiler \\
& compiler GPL Fortran \& Intel Fortran \\
\hline & Mac: GPL Fortran \\
\hline
\end{tabular}

Table 2.2: Different compiling options used in SPHYSICS (Gómez-Gesteira, et al. 2010). 
As mentioned before, SPHYSICS code is originally written for Newtonian fluid problems hence some modifications were necessary in the code. These changes will be comprehensibly explained in Chapter 5; however some of the compiling options presented above used for the models simulated in this research. For example, predictorcorrector time integration scheme is selected with cubic spline kernel function. For the boundary conditions, dynamic B.C. are applied and as for density re-initialization; moving least square method is implemented. The main part of the modifications in the code is implemented on the viscosity treatment section of the code with some minor changes applied in the input generator which are shown in Appendix A.

\subsection{Conclusion}

Prediction of equilibrium beach profiles and overall slope angle of high density tailings at a laboratory scale received attention from various researchers in the past (Fitton et al. 2006; McPhail et al. 2008). Henriquez and Simms (2009) studied the applicability of Lubrication Theory to predict the overall slope angle of the deposit and observed that the angle is scale dependent. Mizani et al. (2013) studied the relation between rheology and deposition geometry and proved that the deposition rate has a significant effect on the final profile. During the deposition, it was observed in the field that the tailings experience several flow states and it is difficult to predict the changes in the flow behaviour (Mizani et al. 2013).

The current work further investigates the effect of deposition rate and initial water content on the equilibrium profile using Bulyanhulu and Val D'Or tailings where the 
yield stress values are measured using slump tests. Also, three dimensional pour tests are conducted to study the flow behaviour of high density tailings.

SPH has been successfully applied to modelling free surface flow problems of nonNewtonian fluids (Zhu et al. 2010; Hosseini et al. 2007; Capone et al. 2010). Present work studies the depositional behaviour of high density tailings at a small scale and this mesh-free numerical method is selected for the purpose.

Rheological model had been modified and validated by the experimental work presented in the following chapter as well as in Henriquez and Simms (2009). The fluid is treated as a Bingham fluid and the 'bi-viscosity' model is selected for its representation since the model defines a greater viscosity value in the un-yielded region instead of having no deformation; therefore the transition between the regions is smoother. Also, with the combination of Equation of State and Dynamic Boundary Conditions, computational time would diminish considerably. 


\section{Chapter: Experimental Methods}

\subsection{Property of Tailings}

The experiments are conducted with gold tailings from Bulyanhulu mine located in the North Tanzania, Africa and Val D'Or mine located in Quebec, Canada. Both of the tailings are collected on site. The Bulyanhulu gold tailings were shipped to Carleton University in plastic buckets where the Val D’Or tailings shipped in large barrels.

The initial gravimetric water content of the Bulyanhulu tailings is $38 \%$ which is also the pumping water content at the site. The tailings settled during transportation due to the vibration and arrived at the university with a layer of water on top. This water is used to mix the tailings at the desired water content. On the other hand, Val D'Or tailings are originally slurry tailings and their water content is approximately $70 \%$. The tailings were completely settled in the barrel and the water on top is used to mix the tailings.

The general characteristics of both tailings are presented below. The geotechnical properties of Val D'Or tailings display some variations. The first batch of tailings was only collected from the top of the barrel where finer particles are located. However, pour tests showed that these tailings had usually low yield stress values. As a result, another barrel was opened and the mixed completely before collected samples for the pour tests. To make sure that the particle distribution is uniform, hydrometer tests were performed to the material collected from the top, middle and the bottom section of the barrel. 


\begin{tabular}{|c|c|c|c|}
\hline & Bulyanhulu & $\begin{array}{c}\text { Val D’Or } \\
\text { (Batch 1) }\end{array}$ & $\begin{array}{c}\text { Val D'Or } \\
\text { (Batch 2) }\end{array}$ \\
\hline Specific Gravity & 2.8 & 1.17 & 2.9 \\
\hline Liquid Limit (\%) & 24 & 33 & 27 \\
\hline Plastic Limit (\%) & 18 & 28 & 20 \\
\hline
\end{tabular}

Table 3.1: The geotechnical properties of tailings

Bulyanhulu tailings and Val D'Or tailings were used for both single layer deposition tests using a flume, while only Val D’Or tailings are used for multi-layer deposition test. The rheological properties of these materials are characterized by using slump tests.

\subsection{The measurement of Yield stress}

Concentrated mineral tailings exhibit non-Newtonian flow behavior meaning that they possess a yield stress. The yield stress $\left(\tau_{\mathrm{y}}\right)$ is a critical shear stress value which must be exceeded before irreversible deformation and flow can occur. Significant amount of work has been completed on the measuring of yield stress values (Jewell and Fourie 2006). Probably, the most simple and on-site yield stress measurement technique is the slump test. Originally used to decide the empirical flow characteristics of fresh concrete, the method was adopted by Pashias et al. (1996) to determine the yield stress of mineral suspension.

The test basically consists of an open-ended cylinder situated on top of a flat surface with the test material inside, then quickly lifting the cylinder and allowing the test material to collapse under its weight. The change in the height of the material is measured. 


\subsubsection{Slump Tests}

\section{Material}

- The testing material; the tailings sample

- Open ended polyvinyl chloride (PVC) cylinder with $19 \mathrm{~cm}$ height and $15.4 \mathrm{~cm}$ diameter (Fig. 3.2).

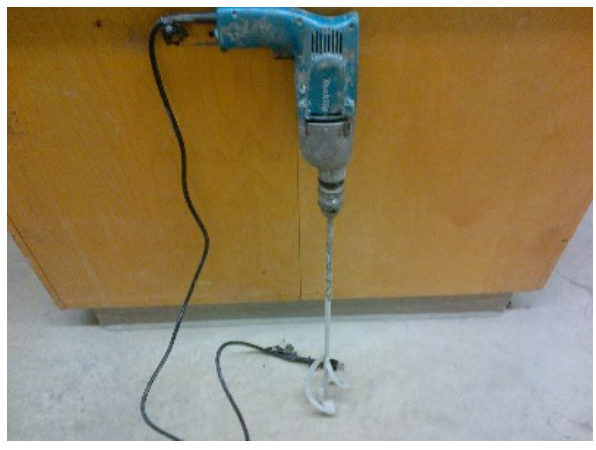

- Distilled water

Figure 3.1: Mixing machine

- Smooth flat surface

- A paint mixer (Fig. 3.1)

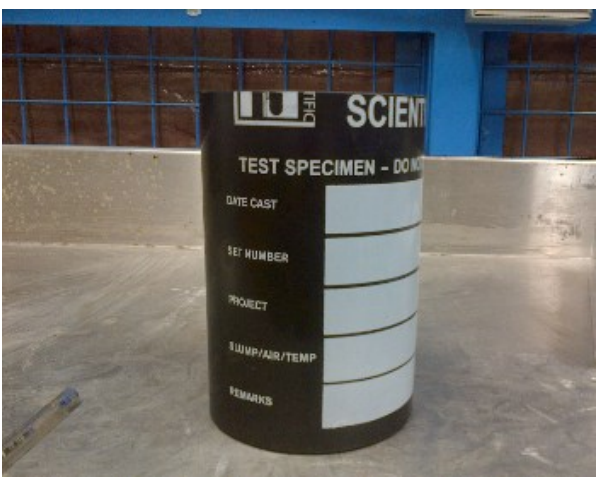

Figure 3.2: Open ended PVC cylinder with 19 $\mathrm{cm}$ height

\section{Experimental Procedure}

Prior to each test, the material was mixed in a bucket for approximately ten minutes. After the material is completely blended, the cylinder was placed on a smooth flat surface and the material was poured into the cylinder. Then the cylinder is quickly lifted and let the material collapse under its own weight. The height difference after the deformation of the tailings is recorded as seen in Figure 3.3. 

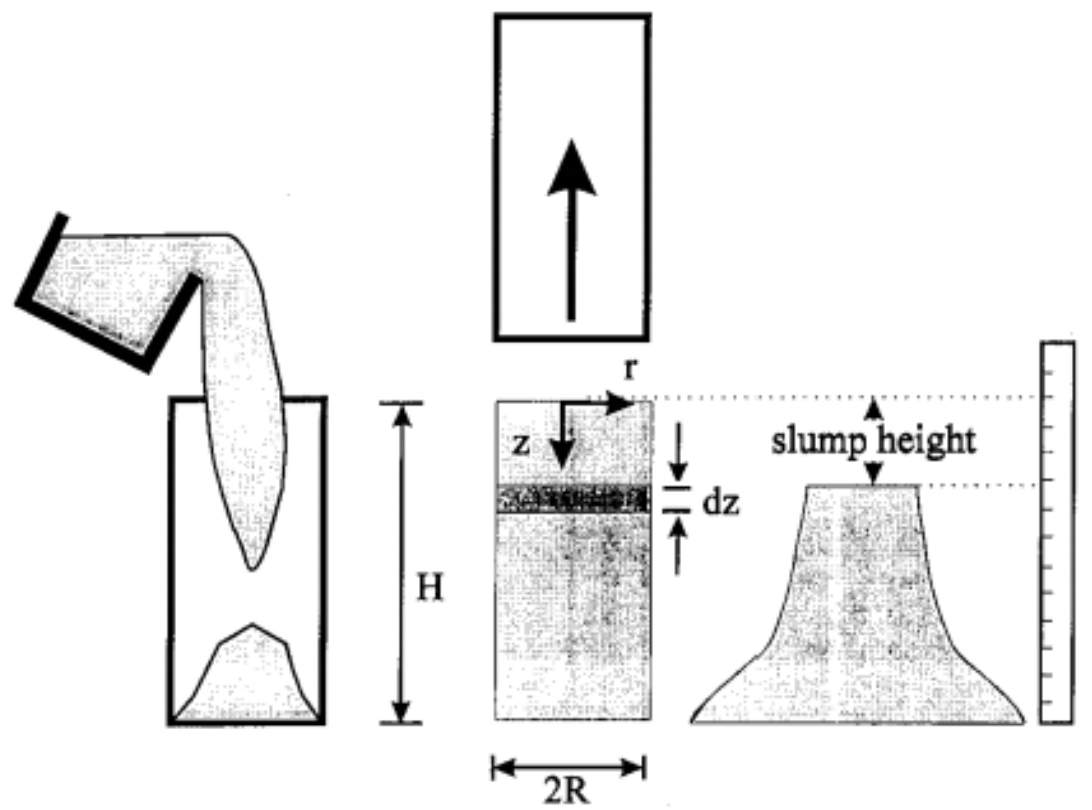

Figure 3.3: The schematic diagram of slump test (Pashias, et al. 1996)

Pashias, et al. (1996) suggested a relationship between the dimensionless yield stress and dimensionless height using the slump test. This relationship can be formulated as follows:

$$
\left.\tau_{y}^{\prime}=\frac{1}{2}-\frac{1}{2} \sqrt{S^{\prime}}\right)
$$

where $\mathrm{S}^{\prime}$ is the dimensionless height (slump height/H) and $\tau_{\mathrm{y}}$ is the dimensionless yield stress. The yield stress is calculated by multiplying the dimensionless yield stress by $\rho g H$ where $\rho$ is the density, $g$ is the gravitational acceleration and $H$ is the full height if the cylinder. 


\subsection{Settling Test}

The tailings undergo hindered settling after or even during deposition. The volume of tailings decreases with time due to self-weight consolidation and settling tests are conducted to determine the change which is estimated by recording the movement in the tailings/water interface.

A total of two settling tests were conducted in order to determine the settling rate of the material after 24 hours. The tailings are poured into a cylinder with $6.5 \mathrm{~cm}$ diameter and $42.2 \mathrm{~cm}$ height. For similar tailings, the diameter of the cylinder has been shown not to affect the rate of consolidation. The settlement test is only applied to Val D'Or tailings since this material used for multilayer depositions. The settlement test is only used to determine i) the final density after the initial settling, and ii) the time it takes for this density to be reached. It was found this final density is fairly insensitive to the height for heights less than $0.8 \mathrm{~m}$. The details of this test will be explained in the following chapters but it should be noted that each layer was left to settle for 24 hours to gain sufficient strength before depositing the next layer on top.

\subsection{Particle Size Distribution}

The particle size distribution was determined by using the hydrometer test which is conducted according to ASTM standard D 422-63 (1990) "Standard Test Method for Particle-Size Analysis of Soil”. 


\subsection{Flume Test}

In the literature, the flume test has been used to study the depositional behaviour of dewatered mine tailings in laboratory scale by several authors (Sofrá and Boger (2002); Henriquez and Simms (2009); Mizani et al. 2013). The flume consists of a flow channel with a reservoir attached to it (Figure 3.4). The gate is located between the reservoir and the flow channel, and the material is filled in the reservoir when the gate is down. Then, the gate is quickly lifted allowing the material to flow down until it comes to a rest. Instead of a gate, a pipe is selected for deposition of the tailings into the flume. The pipe is connected to a pump and tied to the gate wall; the material is poured into the channel from the pipe.

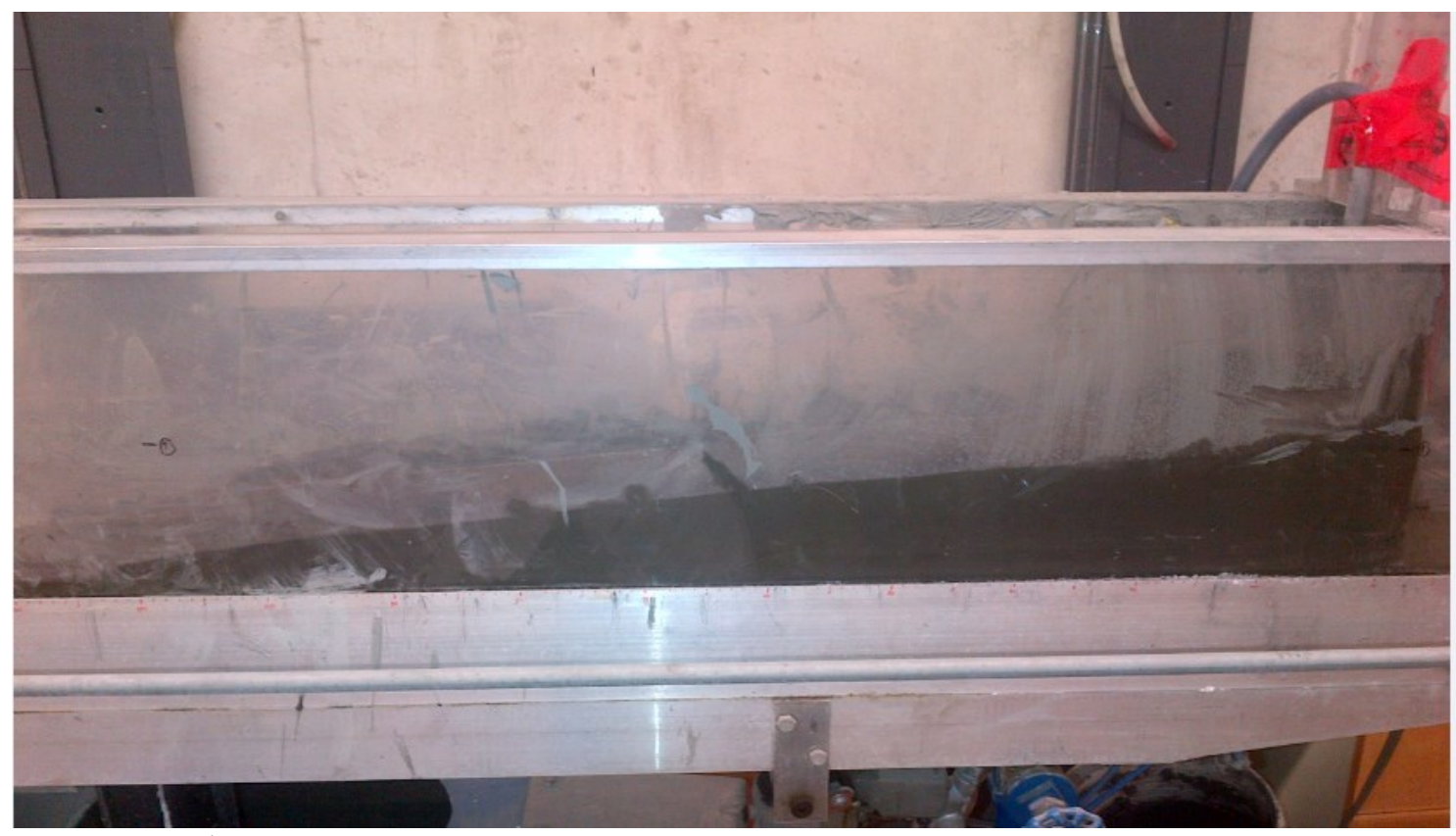

Figure 3.4: Flume apparatus

For the purpose of this research, the flume test was conducted using both Bulyanhulu and Val D’Or (material from the first batch) high density tailings. These tests were 
performed for single layer deposition on a horizontal plane with different flow rates and volumes.

\subsubsection{Experimental Procedure}

\section{Materials:}

- $\quad$ The testing material

- $\quad$ Flume apparatus with acrylic side walls with dimensions (in $\mathrm{cm}$ ) of:

\begin{tabular}{|c|c|c|}
\hline Length & Height & Width \\
\hline 243 & 30 & 15.2 \\
\hline
\end{tabular}

A paint mixer

- A measuring tape

- $\quad 7.3 \mathrm{~mm}$ tube

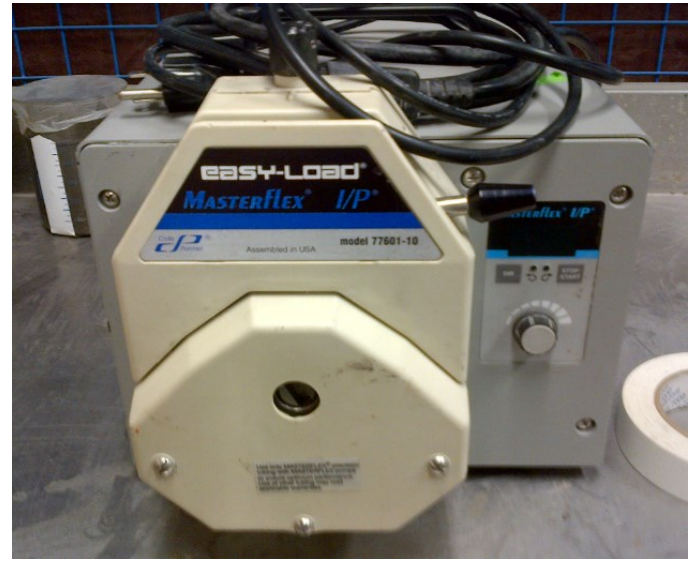

Figure 3.5: The pump

- A pump (Masterflex model

77601-10, Fig 3.5)

- $\quad$ Protective equipment

\section{Experimental Procedure:}

For the purpose of this study, total of 25 flume tests were performed in single layers with different deposition rates and volumes (Table 3.1). Prior to each test, the material is mixed with the paint mixed in buckets for approximately 10 minutes, the density and yield stress values (obtained from slump tests) were recorded. The tailings then pumped 
into the channel by using the pipe with a diameter of $7.3 \mathrm{~mm}$ which was installed $20 \mathrm{~cm}$ above the bottom of the flume.

\begin{tabular}{|c|c|c|c|c|}
\hline Material & Test & $\begin{array}{c}\text { Flow rate } \\
\text { (LPM) }\end{array}$ & Angle & Volume (L) \\
\hline \multirow{14}{*}{$\begin{array}{l}\text { Bulyanhulu } \\
\text { tailings }\end{array}$} & 1 & 0.25 & 8.4 & 11.9 \\
\hline & 2 & 0.64 & 6.9 & 13.4 \\
\hline & 3 & 0.77 & 7.5 & 11.8 \\
\hline & 4 & 1.03 & 6.0 & 11.7 \\
\hline & 5 & 1.5 & 5.8 & 11.1 \\
\hline & 6 & 1.25 & 6.6 & 10.1 \\
\hline & 7 & 1.3 & 6.2 & 11.7 \\
\hline & 8 & 1.76 & 5.7 & 11.6 \\
\hline & 9 & 2 & 5.2 & 10.5 \\
\hline & 10 & 2.3 & 5.1 & 11.0 \\
\hline & 11 & 2.5 & 4.4 & 11.7 \\
\hline & 12 & 3 & 4.3 & 9.8 \\
\hline & 13 & 3.3 & 4.0 & 9.2 \\
\hline & 14 & 4.3 & 3.7 & 9.0 \\
\hline \multirow{11}{*}{$\begin{array}{l}\text { Val D’Or } \\
\text { tailings }\end{array}$} & 1 & 1.58 & 2.9 & 7.6 \\
\hline & 2 & 3.0 & 3.9 & 7.1 \\
\hline & 3 & 2.25 & 4.5 & 7.4 \\
\hline & 4 & 2.28 & 4.3 & 7.0 \\
\hline & 5 & 3.3 & 3.5 & 6.7 \\
\hline & 6 & 3.45 & 2.6 & 6.0 \\
\hline & 7 & 2.75 & 2.7 & 6.5 \\
\hline & 8 & 2.4 & 4.0 & 5.6 \\
\hline & 9 & 3.05 & 3.7 & 6.2 \\
\hline & 10 & 1.62 & 6.0 & 6.0 \\
\hline & 11 & 2.45 & 4.3 & 6.5 \\
\hline
\end{tabular}

Table 3.1: Summary of flume tests

The length of the flow and the depth of the material is measured at different locations (every $5 \mathrm{~cm}$ along the flume and decreased to $1 \mathrm{~cm}$ at the end) after the flow is come to a complete stop. All the tests are performed in the room temperature; between 21 to $24{ }^{\circ} \mathrm{C}$.

The overall angle of the stack is calculated using the formula below: 


$$
\theta_{r}=\tan ^{-1}\left(\frac{H_{f}-H_{i}}{L}\right)
$$

where $H_{f}$ is the depth at the toe, $H_{i}$ is the depth near the deposition point and $L$ is the horizontal distance between these two points.

The equation for the equilibrium profile of a slow spreading Bingham fluid on a flat bed is defined by Liu and Mei (1989) is described below;

$$
\tau_{y}\left(x-x_{0}\right)=\frac{\rho g}{2}\left(h^{2}-h_{0}^{2}\right)
$$

where $\tau_{y}$ is the yield stress, $g$ is the gravitational acceleration, $\rho$ is the density, $h$ is the depth of the flow at a particular $x$ which is measured perpendicular to the horizontal plane and $h_{0}$ is the depth of the flow at $x_{0}$.

\subsection{Pour Test}

Pour tests are multi-layer deposition tests, intended to approximate the 3D deposition of mine tailings on a small scale. Three tests consist (Table 3.2) of depositing a certain volume of tailings poured onto a flat surface using a pipe and a pump, forming conical stacks which will grow upwardly.

\begin{tabular}{|c|c|c|}
\hline Pour test \# & Material (Val D'Or) & Flow rate (LPM) \\
\hline 1 & Batch 1 & 0.28 \\
\hline 2 & Batch 1 & 4.0 \\
\hline 3 & Batch 2 & 3.7 \\
\hline
\end{tabular}

Table 3.2: Summary of pour tests

A new layer is deposited after the previous layer stop settling and before start desiccating. The geometry of the stack was recorded for each settling layer and the new deposited layer to make sure there are no failures occurs in the settled layer. These tests 
are conducted on a flat $1 \mathrm{~m}$ by $1 \mathrm{~m}$ Plexiglas surface (Figure 3.6) where the tailings are pumped from a pipe with a $7.3 \mathrm{~mm}$ diameter above the deposition point. The pipe was positioned approximately $23 \mathrm{~cm}$ above the surface of the tailings allowing them to flow radially. In order to minimize the evaporation rates, the tailings were covered with a plastic sheet demonstrated in Figure 3.6.

\subsubsection{Experimental Procedure}

\section{Materials}

- The tailings sample

- Plexiglas box of $1 \mathrm{~m}$ by $1 \mathrm{~m}$ (Fig.

- A paint mixer

- A measuring tape

- A pipe with $7.3 \mathrm{~mm}$ diameter

- A pump (Masterflex model

77601-10, Fig. 3.5)

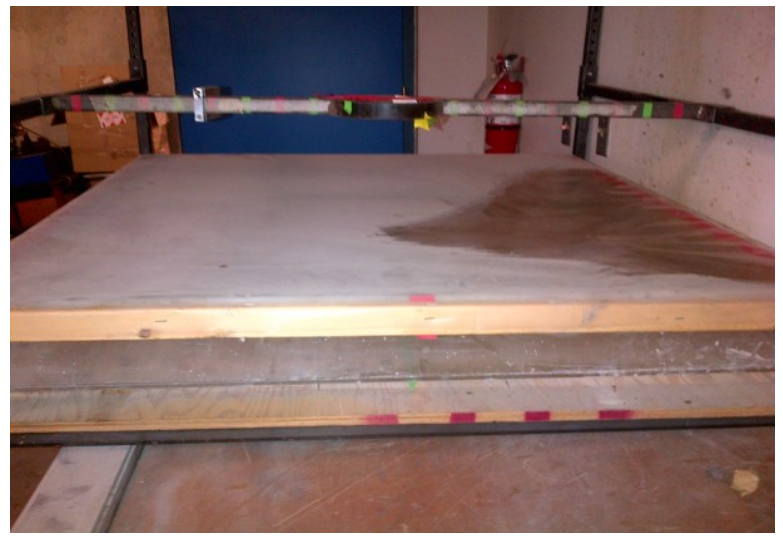

Figure 3.6: Plexiglas box with plastic sheet cover on top.

- Plastic sheet to cover

- Personal protective equipment

\section{Experimental Procedure}

Prior to each test, the density, water content and the yield stress values of the tailings are determined and the material is mixed in buckets for approximately 10 minutes. Once 
the material is thoroughly mixed, exactly $1.3 \mathrm{~L}$ volumes of tailings are poured onto the box through a pipe.

Once the material tops flowing visually, the topography were measured in North $(\mathrm{N})$, South (S), West (W) and East (E) directions by dropping a measurement tape from an overlying grid at each $5 \mathrm{~cm}$ intervals. Tailings are left to settle for 24 hours and to minimize the evaporation; the box is covered up with a plastic sheet.

The next layer is then poured onto the settled layer and the new topography was measured. The results are then compared with the Lubrication theory; first layer geometry predictions were determined from Equation 3.3 and for the successive layers the following equation was employed.

$$
h^{\prime}-h_{0}{ }^{\prime}+\ln \left(1-h^{\prime}\right)=x^{\prime}-x_{0}{ }^{\prime}
$$

where;

$$
\begin{gathered}
h=h^{\prime}\left(\frac{\tau_{y}}{\rho g \sin \theta}\right) \\
x=x^{\prime} \cot \theta\left(\frac{\tau_{y}}{\rho g \sin \theta}\right)
\end{gathered}
$$

The angle used in the equations are represents the underlying topography, which is obtained from the predicted initial layer. 


\section{Chapter: Experimental Results and Discussion}

The following section discusses the results from the experimental work review in Chapter 3; including the particle size distribution, slump tests, flume experiments and pour tests.

\subsection{Particle Size Distribution}

\begin{tabular}{|c|c|c|c|}
\hline & Bulyanhulu & $\begin{array}{c}\text { Val D'Or } \\
\text { (Batch 1) }\end{array}$ & $\begin{array}{c}\text { Val D'Or } \\
\text { (Batch 2) }\end{array}$ \\
\hline Specific Gravity & 2.8 & 1.2 & 2.9 \\
\hline Liquid Limit (\%) & 24 & 33 & 27 \\
\hline Plastic Limit (\%) & 18 & 28 & 20 \\
\hline
\end{tabular}

Table 4.1: Geotechnical properties of tested tailings

Particle size distribution is only applied to the material from the second batch of Val D'Or tailings to make sure that the particle distribution in the barrel is uniform after mixing the material inside the barrel. The samples were taken from the top, middle and the bottom sections of the barrel and the results demonstrate that the particle size distribution in the barrel is in fact uniform. The results of particle size distribution of Bulyanhulu tailings obtained from Mizani (2010) is also added in Figure 4.1 to give a better understanding of the material used in the flume tests. 


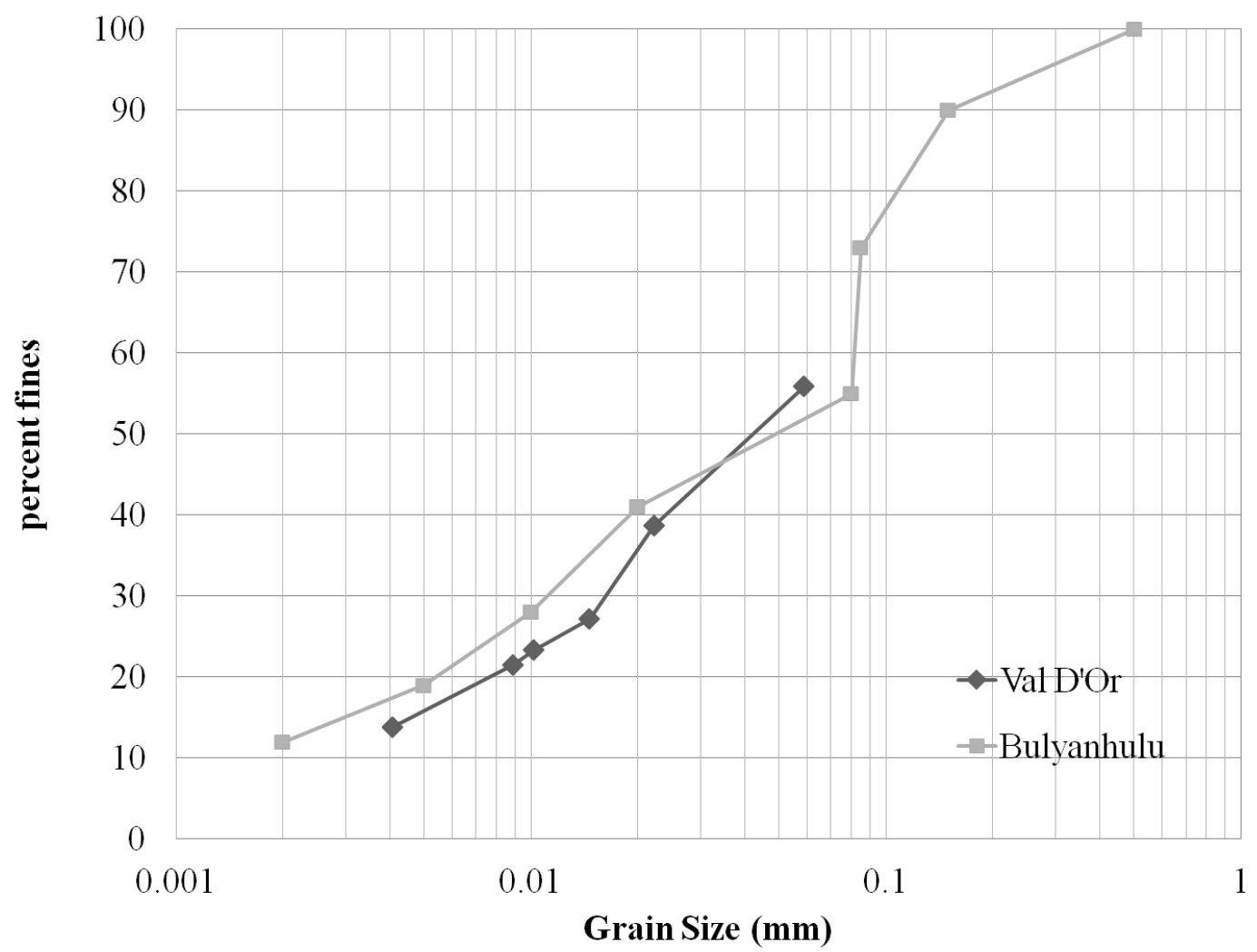

Figure 4.1: Hydrometer test results for the Bulyanhulu and Val D'Or tailings (material collected from the top section of the barrel).

As demonstrated in the figure, grain size of Val D'Or gold tailings varies between 0.004 to $0.05 \mathrm{~mm}$ where the average size $\left(d_{50}\right)$ is $0.03 \mathrm{~mm}$. The grain size of Bulyanhulu tailings ranges from $1 \mu \mathrm{m}$ to $0.5 \mathrm{~mm}$ and the average size is approximately $35 \mu \mathrm{m}$.

\subsection{Yield Stress}

The dimensionless slump height can be calculated by dividing the difference in height of the slumped material by the initial height of the cylinder which is $19 \mathrm{~cm}$ in the experiments. Following figure displays the relationship using the slump tests for both Bulyanhulu and Val D’Or high-density tailings. 


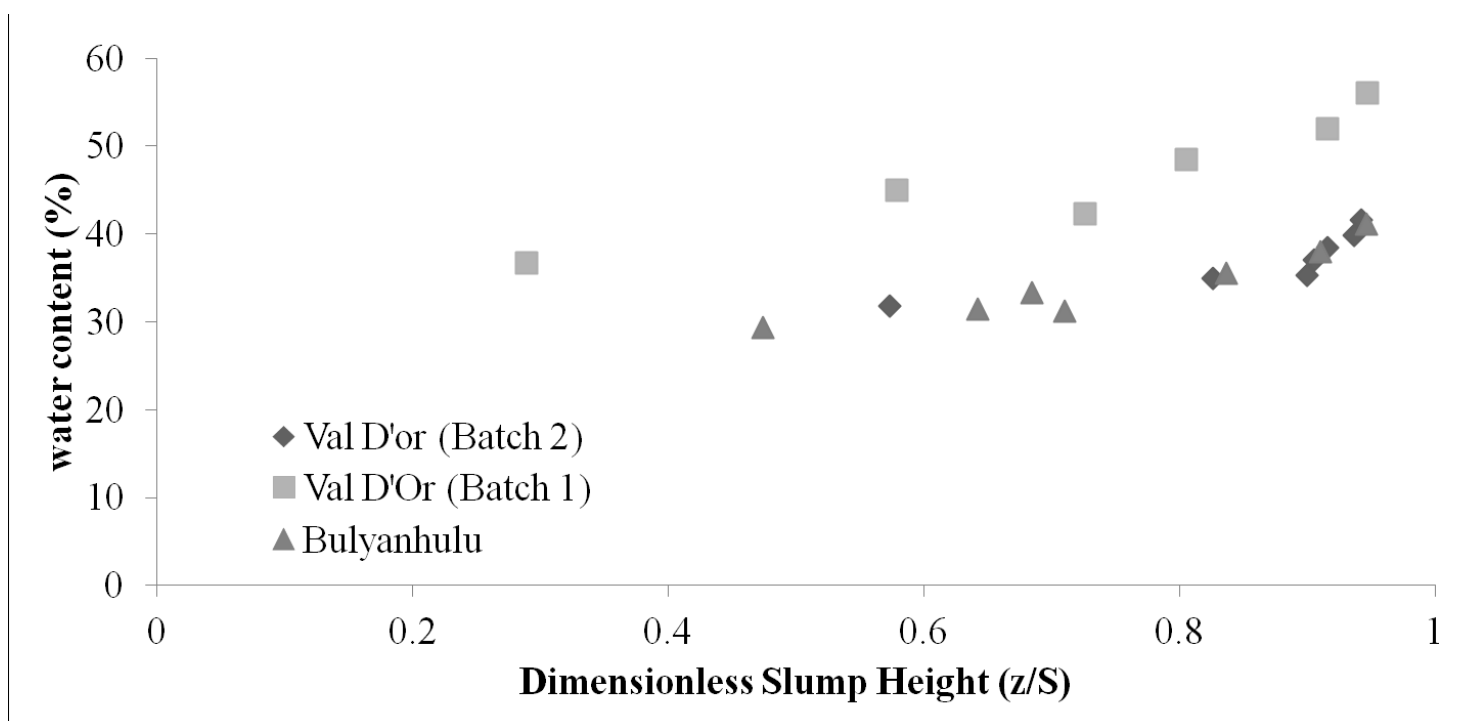

Figure 4.2: Dimensionless slump height versus water content.

Several slump tests were conducted in order to determine the strength of the mineral tailings at different water contents. The density values are measured before each tests and Figure 4.3 demonstrates the yield stress values for different water contents.

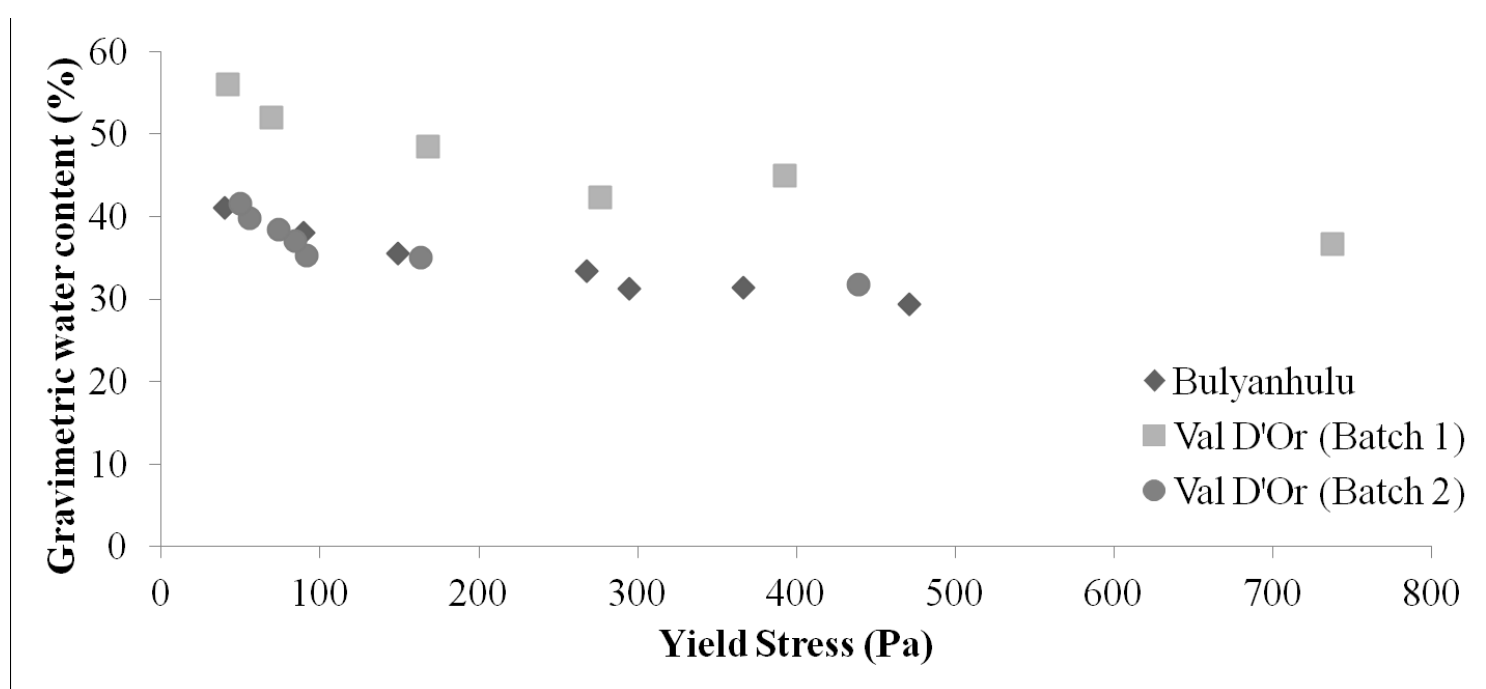

Figure 4.3: Yield stress values versus gravimetric water content

As observed in Figure 4.3, the first batch of Val D'Or tailings have higher yield stress values for the same water contents due to the domination of finer particles. When a 
net inter-particle attraction exists, the yield stress values might increase in the presence of Wan der Waals and electrical double layer forces. Calculated yield stresses are not only dependent on these forces but also dependent on the particle structural considerations such as solids volume fraction, particle size and mean coordination number. At smaller scales, electrochemical forces such as Van der Waals and double layer forces increase therefore suspension with smaller particles tend to have higher yield stresses (Johnson, et al. 2000).

For the second batch of Val D'Or and Bulyanhulu tailings, the yield stress values became sensitive to changes in water content from 35 to $30 \%$. Bulyanhulu and Val D'Or (first batch) were selected for the flume tests and the gravimetric water content of the Bulyanhulu tailings are approximately $38 \%$ which corresponds to a yield stress value of $72 \mathrm{~Pa}$. On the other hand, the first batch of Val D'Or tailings had water content of 55\% therefore the yield stress values are in the region of $50 \mathrm{~Pa}$. The second batch of Val D'Or tailings are utilized in pour tests and the yield stress values are approximately $45 \mathrm{~Pa}$ which corresponds to $39 \%$ of water content.

\subsection{Settling Test}

The initial height of the first batch of Val D'Or tailings is $30.2 \mathrm{~cm}$ where the initial height of the second batch is $35 \mathrm{~cm}$. The measurements were taken using a ruler and to minimize the evaporation a cap was placed on top of the cylinder.

It should be noted that the layer were left to settle for 24 hours on multilayer deposition tests. The water content measured from the settlement test of the first batch of Val D'Or tailings dropped from $50 \%$ to $45 \%$ in 24 hours. In pour tests the previous 
settled layers should have enough strength to support the next deposited layer however, the tests conducted with this material showed that 24 hours is not enough for the material to completely settle. As a result, some of the layers failed to carry the new deposited layers.

The gravimetric water content of the second batch was initially measured as $42 \%$ and this value dropped to $33 \%$ in 9.5 hours. The yield stress value corresponding to the final water content is between 200 to $250 \mathrm{~Pa}$ hence 24 hours is long enough for the layer to completely settle and gain strength.

\subsection{Flume Tests}

The following section is divided into two subsections based on the material used in flume tests. For the purpose of this research, flume tests are only applied on single layer depositions on a flat surface. Total of 25 tests were conducted using the flume apparatus.

\subsubsection{Bulyanhulu Tailings}

Total of 14 single layer deposition tests were performed in order to determine the effect of the deposition flow rate on the final profile. The tests are summarized in Table 4.2. 


\begin{tabular}{|c|c|c|c|c|}
\hline Test & Flow rate (LPM) & $\begin{array}{c}\text { Yield stress } \\
\text { according to } \\
\text { Figure 4.3 }(\mathrm{Pa})\end{array}$ & Angle & Volume (L) \\
\hline 1 & 0.25 & 72 & 8.4 & 11.9 \\
\hline 2 & 0.64 & 72 & 6.9 & 13.4 \\
\hline 3 & 0.77 & 72 & 7.5 & 11.8 \\
\hline 4 & 1.03 & 72 & 6.0 & 11.7 \\
\hline 5 & 1.5 & 72 & 5.8 & 11.1 \\
\hline 6 & 1.25 & 72 & 6.6 & 10.1 \\
\hline 7 & 1.3 & 72 & 6.2 & 11.7 \\
\hline 8 & 1.76 & 72 & 5.7 & 11.6 \\
\hline 9 & 2 & 72 & 5.2 & 10.5 \\
\hline 10 & 2.3 & 72 & 5.1 & 11.0 \\
\hline 11 & 2.5 & 72 & 4.4 & 11.7 \\
\hline 12 & 3 & 55 & 4.3 & 9.8 \\
\hline 13 & 3.3 & 51 & 4.0 & 9.2 \\
\hline 14 & 4.3 & 45 & 3.7 & 9.0 \\
\hline
\end{tabular}

Table 4.2: Summary of flume tests conducted with Bulyanhulu tailings.

The final geometry of some of the flume tests are presented in Figure 4.4. As the flow rate increases, the angle of the deposition decreases which varied from $8.4^{\circ}$ to $3.7^{\circ}$. For the tailings deposited at 2.5 LPM or higher, no profile changes are observed (Figure 4.6 and Figure 4.7) 


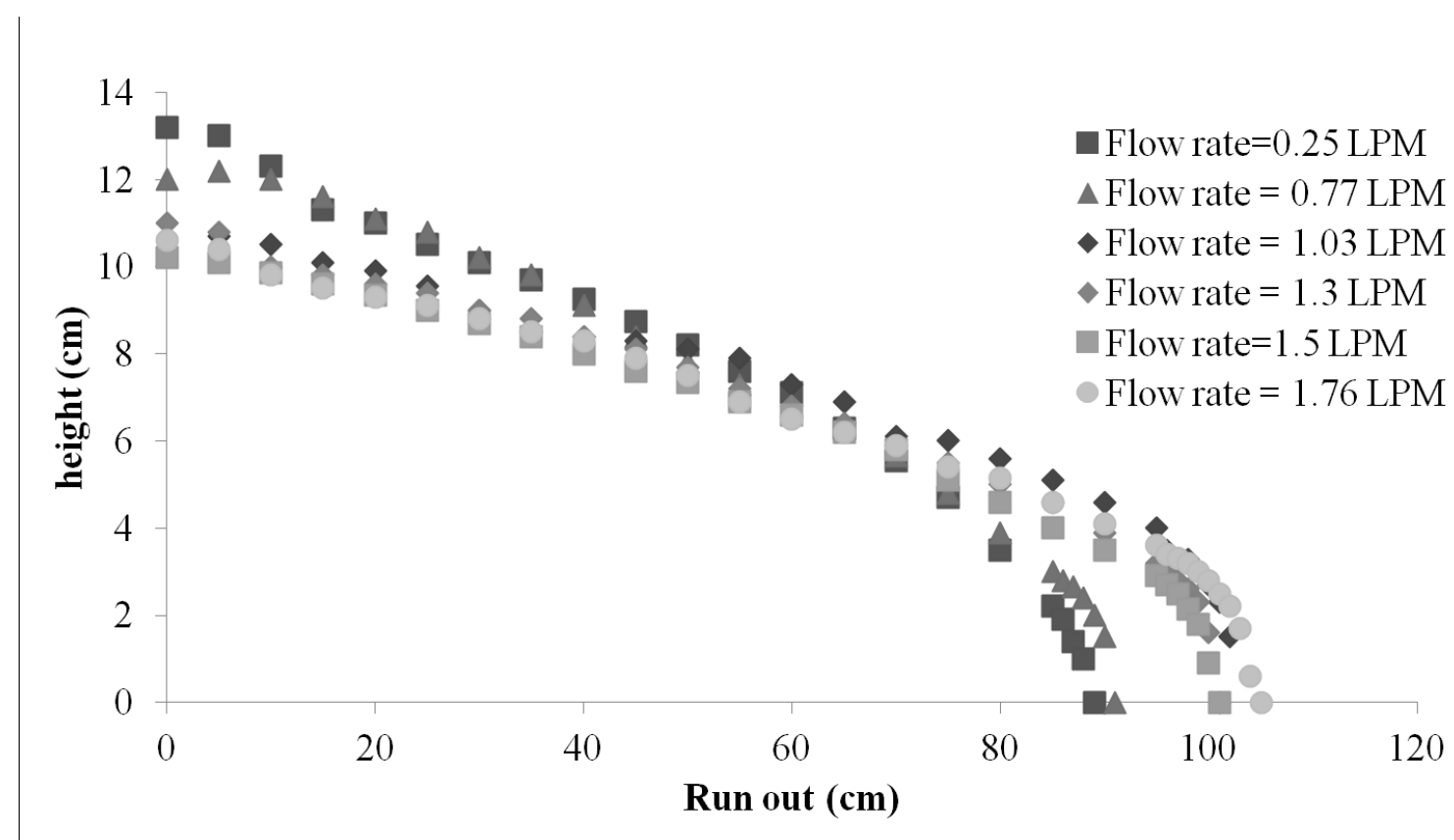

Figure 4.4: Six single layer flows at different flow rates with volumes approximately $11 \mathrm{~L}$ (gravimetric water content is $37 \%$, yield stress is $71.47 \mathrm{~Pa}$ ).

As it can be observed form the Figure 4.4, for the approximately same amount of volume, the final profile of the slowest flow rate has the shortest run out distance and the maximum height of tailings. For the 0.25 LPM, the yield stress increased significantly at the end of the experiment ( $72 \mathrm{~Pa}$ to $150 \mathrm{~Pa}$ ) due to settlement occurred during the test (Figure 4.5). Hence, there is a significant difference in $h_{o}$ between the measured value and predicted value as it can be seen below. As expected, the largest angle is observed in 0.25 LPM test, $8.4^{\circ}$. 


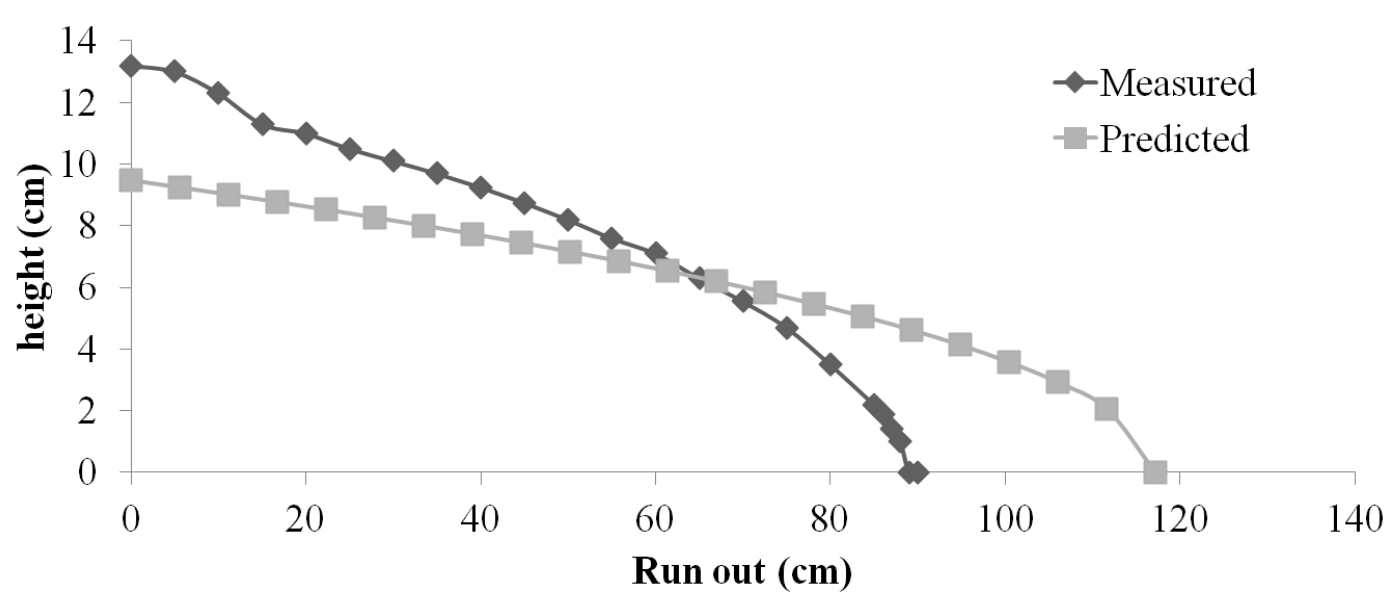

Figure 4.5: The final profile of Bulyanhulu tailings at $38 \%$ water content with a flow rate of 0.25 LPM including LT prediction.

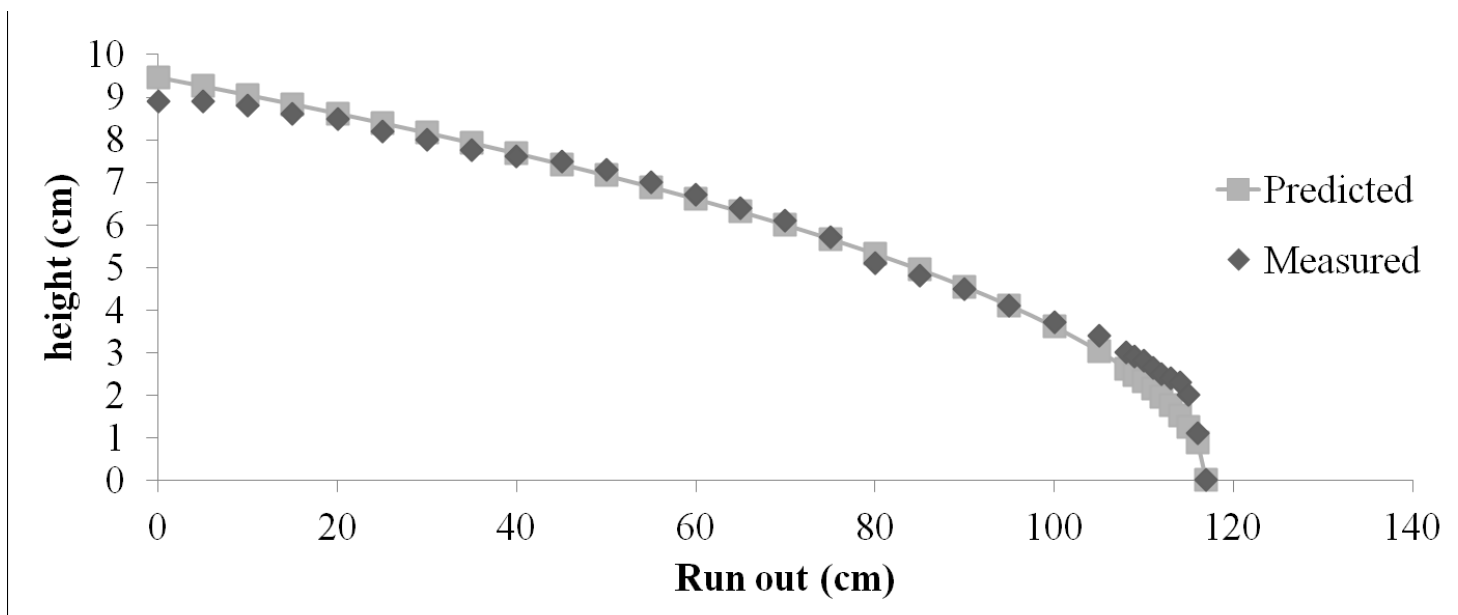

Figure 4.6: Single layer test with a deposition rate of 2.5 LPM compared with LT prediction.

The smallest angle was obtained from the 4.3 LPM test (Figure 4.7). The measured and the predicted shape of the tailings give better correlation for the highest flow rate. 


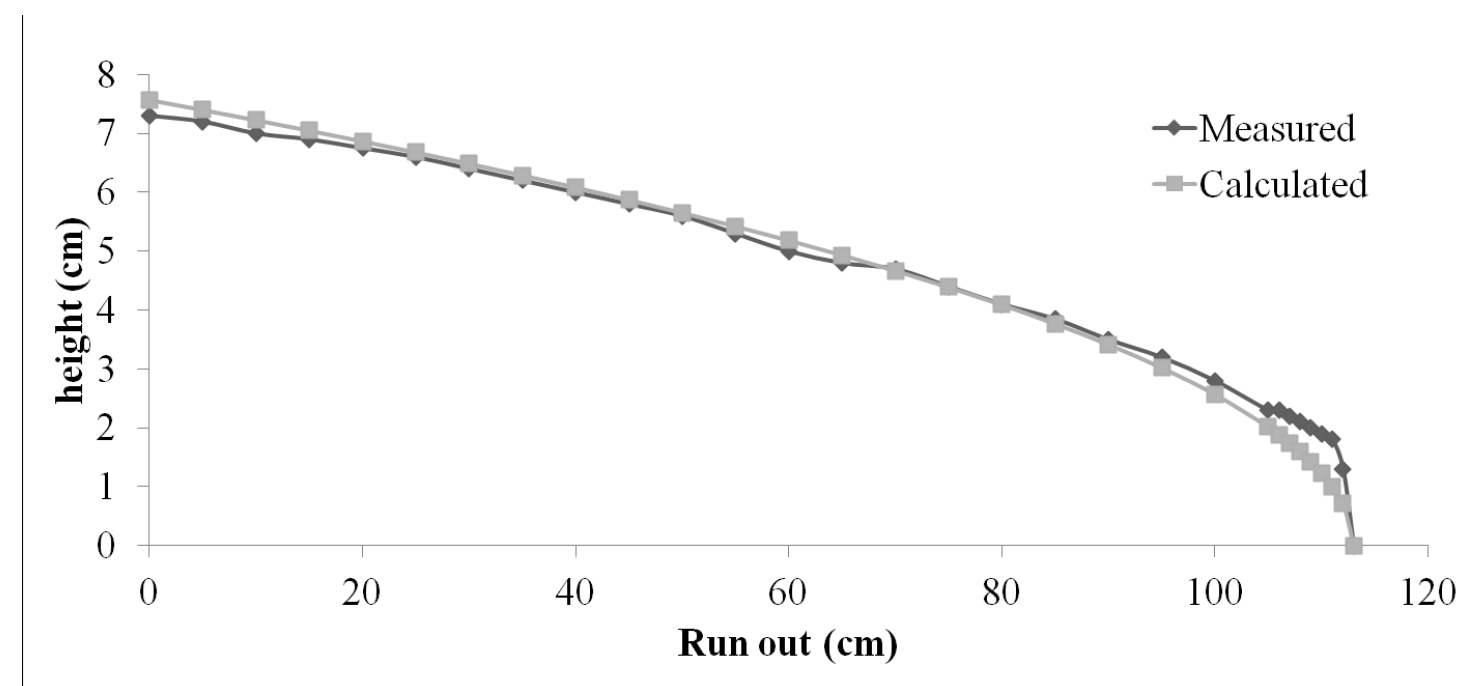

Figure 4.7: The final profile of tailings with a flow rate of 4.3 LPM including LT prediction.

The last three tests conducted using Bulyanhulu tailings with smaller volume and slightly higher gravimetrical water content compared to the first eleven tests. The gravimetric water content of the material is $38.6 \%$ with corresponding yield stress values are approximately $50 \mathrm{~Pa}$. The final geometries of these tailings are presented in Figure 4.8. The deposition rates of these three tests are higher than 2.5 LPM; therefore the predicted geometry from the Equation 3.3 and the measured data gives a good agreement. Best fitted yield stress values calculated using Lubrication Theory equations are demonstrated in Table 4.3. 


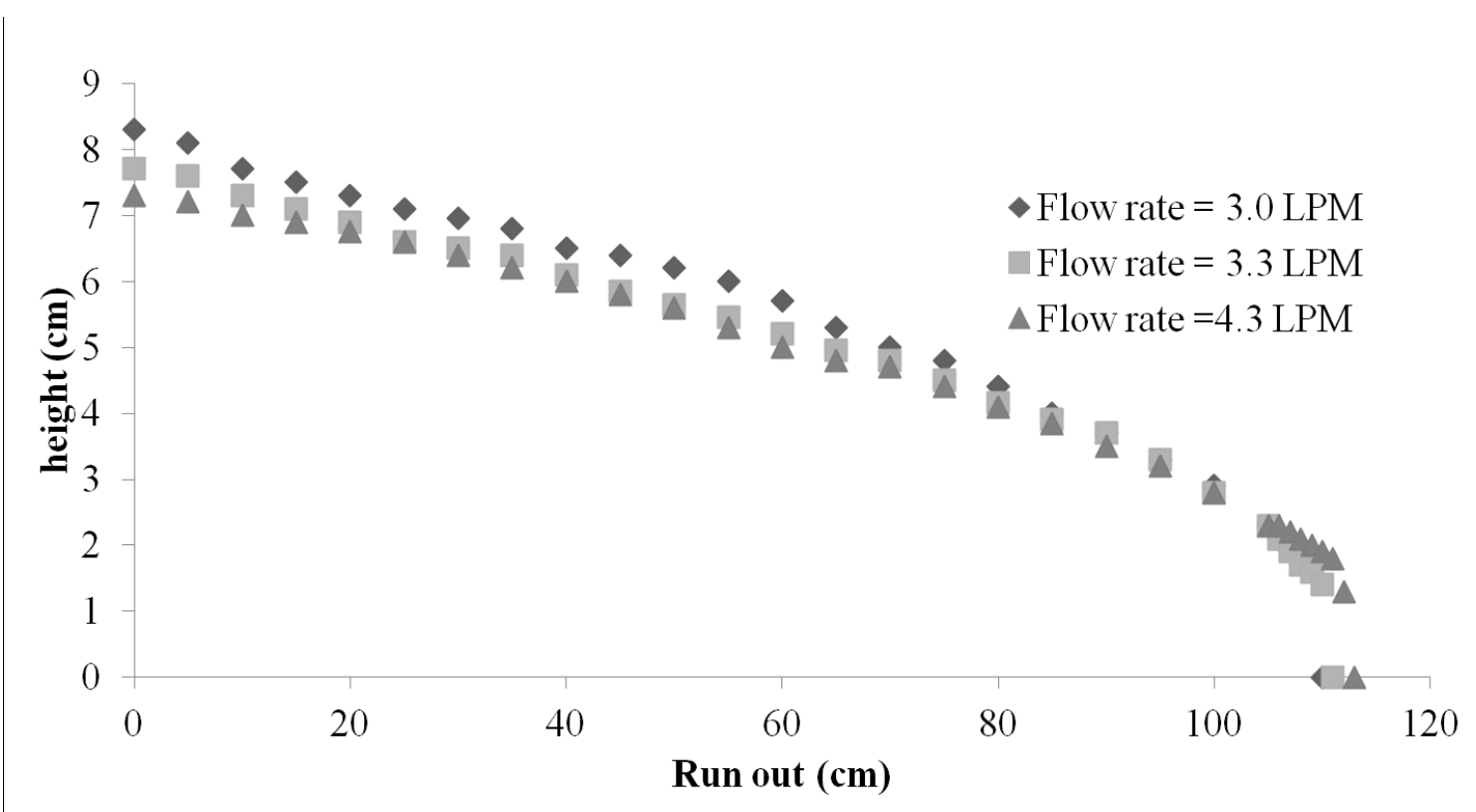

Figure 4.8: Three single layer flows with volumes approximately $9 \mathrm{~L}$ (gravimetric water content is $38 \%$ and yield stress is approximately $50 \mathrm{~Pa}$ )

\begin{tabular}{|c|c|c|c|}
\hline Test \# & Flow rate (LPM) & $\begin{array}{l}\text { Yield stress values obtained } \\
\text { from Slump test (Pa) }\end{array}$ & $\begin{array}{c}\text { Best-fitted yield stress } \\
(\mathrm{Pa})\end{array}$ \\
\hline 1 & 0.25 & 72 & 150 \\
\hline 2 & 0.64 & 72 & 121 \\
\hline 3 & 0.77 & 72 & 120 \\
\hline 4 & 1.03 & 72 & 105 \\
\hline 5 & 1.5 & 72 & 95 \\
\hline 6 & 1.25 & 72 & 98 \\
\hline 7 & 1.3 & 72 & 103 \\
\hline 8 & 1.76 & 72 & 90 \\
\hline 9 & 2 & 72 & 81 \\
\hline 10 & 2.3 & 72 & 80 \\
\hline 11 & 2.5 & 72 & 72 \\
\hline 12 & 3 & 55 & 55 \\
\hline 13 & 3.3 & 51 & 51 \\
\hline 14 & 4.3 & 45 & 45 \\
\hline
\end{tabular}

Table 4.3: Best-fitted yield stress values obtained from LT equations for Bulyanhulu tailings.

Figure 4.9 demonstrates that the best-fitted yield stress values increases as the deposition time increases since the particles start to settle during the deposition. For higher flow rates, calculated yield stress values do not vary from the yield stress values 
obtained from slump test. Increase in the yield stresses may reflect thixotrophic behaviour of tailings as they flow; Mizani, et al. (2013) also found that the density and the yield stress values of the tailings will increase with time of deposition due to the aging process which is linked to settling behaviour (Figure 4.9). It should be noted that the initial measured yield stress values from Mizani et al. (2013) is approximately $30 \mathrm{~Pa}$ and the initial gravimetric water content was somewhat higher (38.4 compared to $37.6 \%$ ).

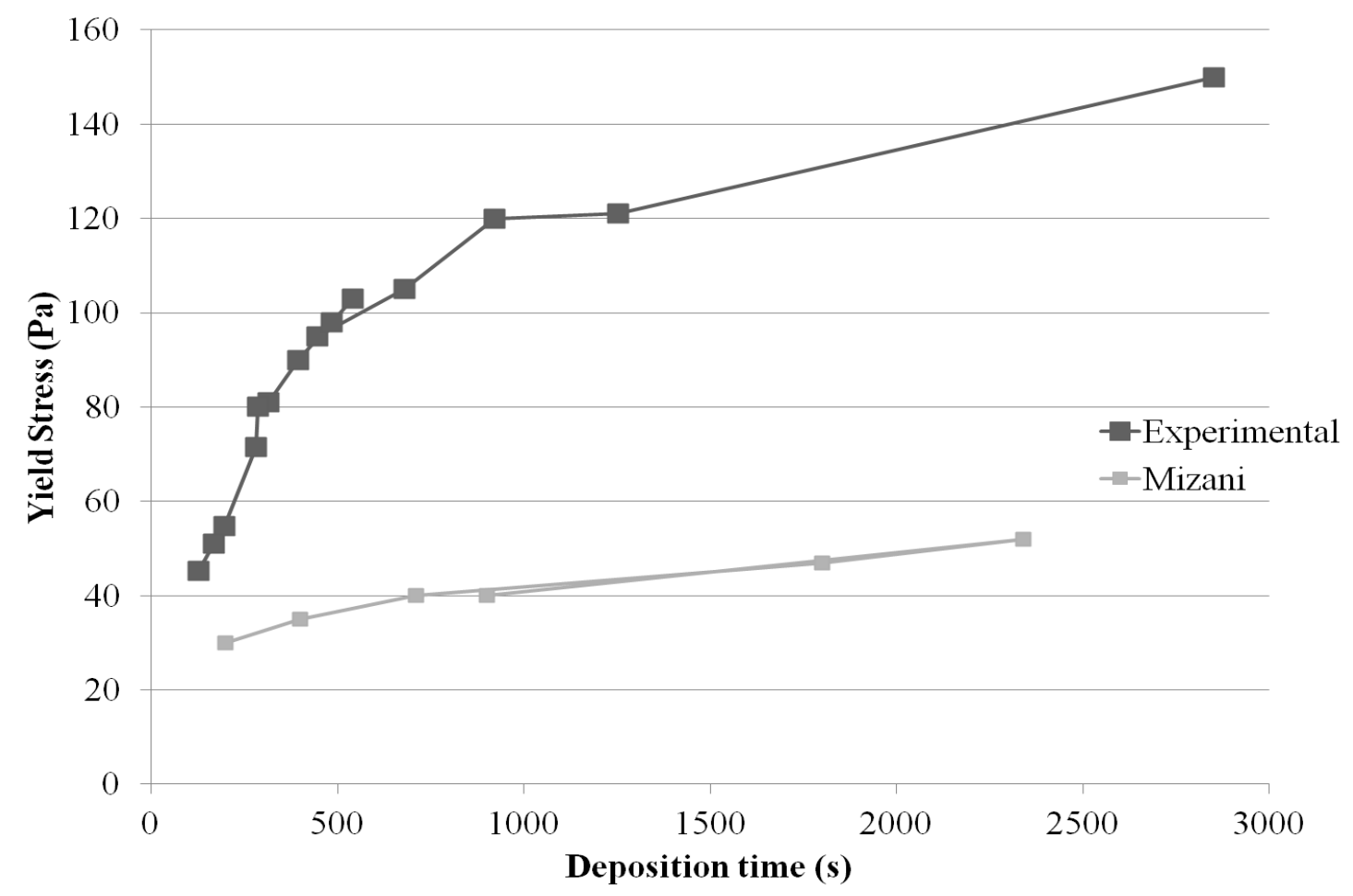

Figure 4.9: Best-fitted yield stress versus deposition time for Bulyanhulu tailings.

\subsubsection{Val D'Or tailings}

The same procedure also applied to Val D'Or tailings but only to the material obtained from the first batch. Originally, this material is slurry tailings with initial gravimetric water content of $70 \%$. However, the water content of the material is reduced so that the material can exhibit a non-Newtonian fluid behavior. For the purpose of the 
research, the water content is decreased to 51 to $55 \%$, hence the corresponding yield stress values varies between 55 to $80 \mathrm{~Pa}$. The only exception is the test case \#10 where the gravimetric water content was lowered to $44.5 \%$; resulting an increase in the yield stress value. The summary of the flume tests are presented below.

\begin{tabular}{|c|c|c|c|c|c|}
\hline Test & $\begin{array}{c}\text { Water content } \\
(\%)\end{array}$ & $\begin{array}{c}\text { Flow rate } \\
(\mathrm{LPM})\end{array}$ & $\begin{array}{c}\text { Yield stress } \\
(\mathrm{Pa})\end{array}$ & Angle & Volume (L) \\
\hline 1 & 53 & 1.58 & 66 & 2.9 & 7.6 \\
\hline 2 & 52 & 3.0 & 79 & 3.9 & 7.1 \\
\hline 3 & 53 & 2.25 & 63 & 4.5 & 7.4 \\
\hline 4 & 52 & 2.28 & 80 & 4.3 & 7.0 \\
\hline 5 & 53 & 3.3 & 61 & 3.5 & 6.7 \\
\hline 6 & 55 & 3.45 & 50 & 2.6 & 6.0 \\
\hline 7 & 55 & 2.75 & 56 & 2.7 & 6.5 \\
\hline 8 & 54 & 2.4 & 62 & 4.0 & 5.6 \\
\hline 9 & 55 & 3.05 & 55 & 3.7 & 6.2 \\
\hline 10 & 45 & 1.62 & 103 & 6.0 & 6.0 \\
\hline 11 & 52 & 2.45 & 75 & 4.3 & 6.5 \\
\hline
\end{tabular}

Table 4.4: Summary of flume tests conducted with Val D'Or tailings.

The test results can be divided into three sections based on the gravimetric water content of the materials. For the Val D'Or tailings with $53 \%$ of water content, the yield stress values obtained from slump tests are in the range of 61 to 66 Pa. Figure 4.10 demonstrates the final profile of two different flume tests conducted with the material sample at the $53 \%$ gravimetric water content. As the flow rate increases, the run out of the material will be longer and the height of the tailings will be lower. 


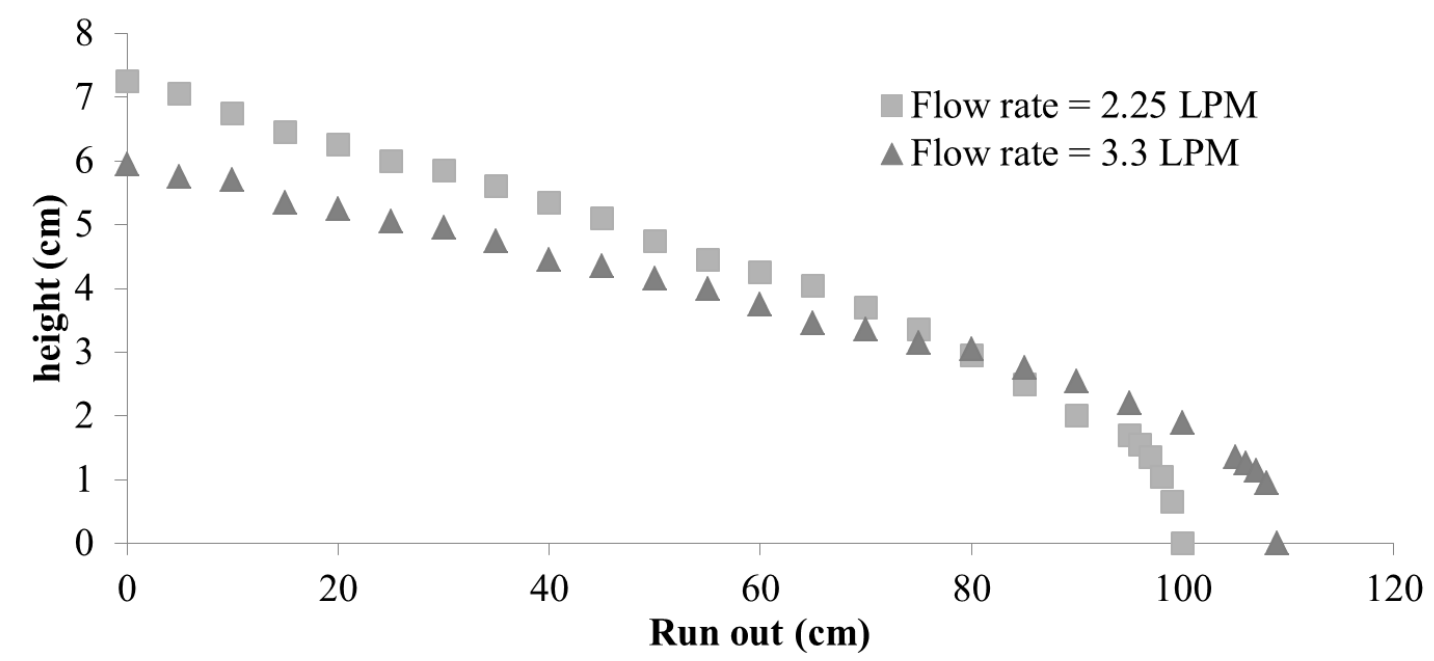

Figure 4.10: Final profile of Val D'Or tailings with 53\% gravimetric water content and roughly $60 \mathrm{~Pa}$ of yield stress.

The results are then compared with the Lubrication Theory based on Equation 3.3 and the results showed that the predicted height of the tailings is higher than the actual measurements. It was mentioned in the previous section that for the same amount of volume of tailings, the run out distance will be longer for the material deposited at a higher flow rate. As it can be observed in Figure 4.10, the material with higher flow rate has longer run out distance. Measured and calculated profiles (using Lubrication Theory equations) of material of the first test are demonstrated in Figure 4.11. 


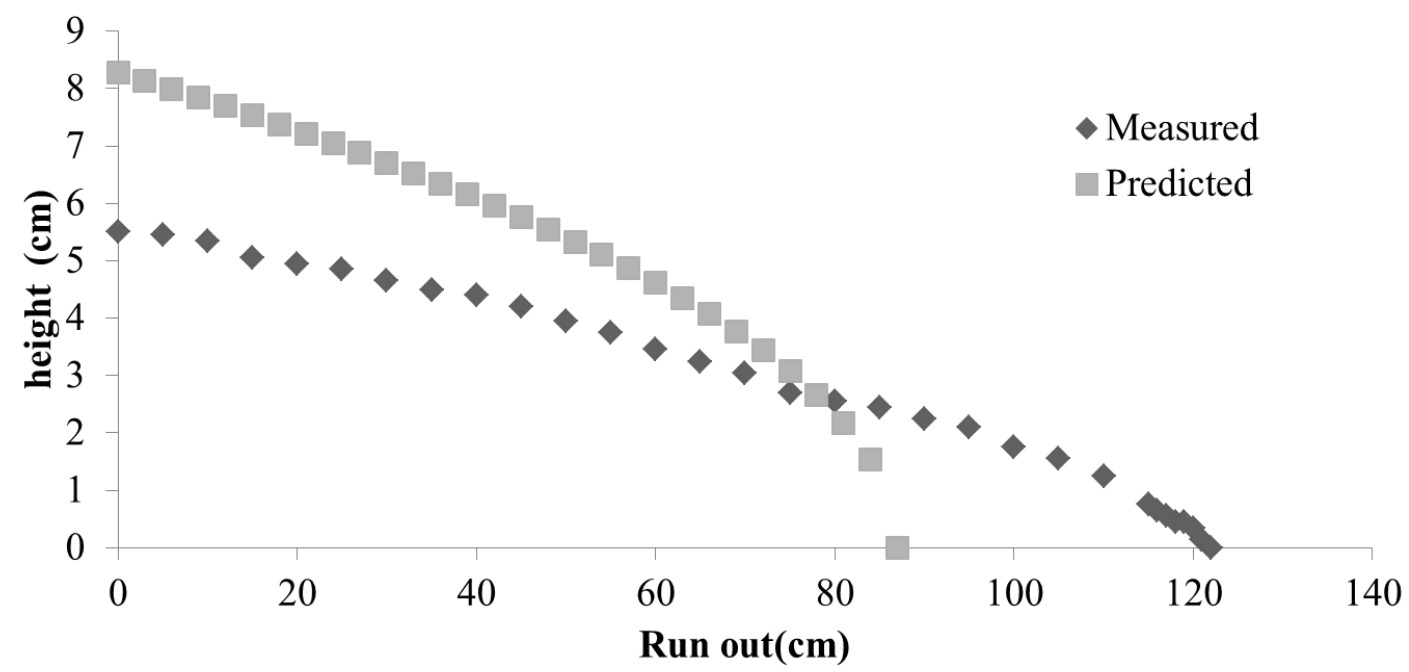

Figure 4.11: The comparison of predicted and the measured final profiles of Val D'Or tailings with a flow rate of $1.58 \mathrm{LPM}, 53 \%$ gravimetric water content and $66 \mathrm{~Pa}$ of yield stress.

The second section of the flume tests are conducted with the tailings at $51-52 \%$ of gravimetric water content.

\begin{tabular}{|c|c|c|c|}
\hline Test \# & Flow rate $(\mathrm{LPM})$ & Yield stress $(\mathrm{Pa})$ & Angle \\
\hline 2 & 3.0 & 79 & 3.9 \\
\hline 4 & 2.28 & 80 & 4.3 \\
\hline 11 & 2.45 & 75 & 4.3 \\
\hline
\end{tabular}

Table 4.5: Summary of flume tests conducted with material at $51-52 \%$ water content

The measured yield stress values are higher, increased up to $80 \mathrm{~Pa}$, the details are provided in Table 4.5. As expected, for the materials deposited with higher flow rates has a smaller angle. Best-fitted yield stress values are also presented in Figure 4.13; as expected the yield stress values increases as the deposition time increases. 


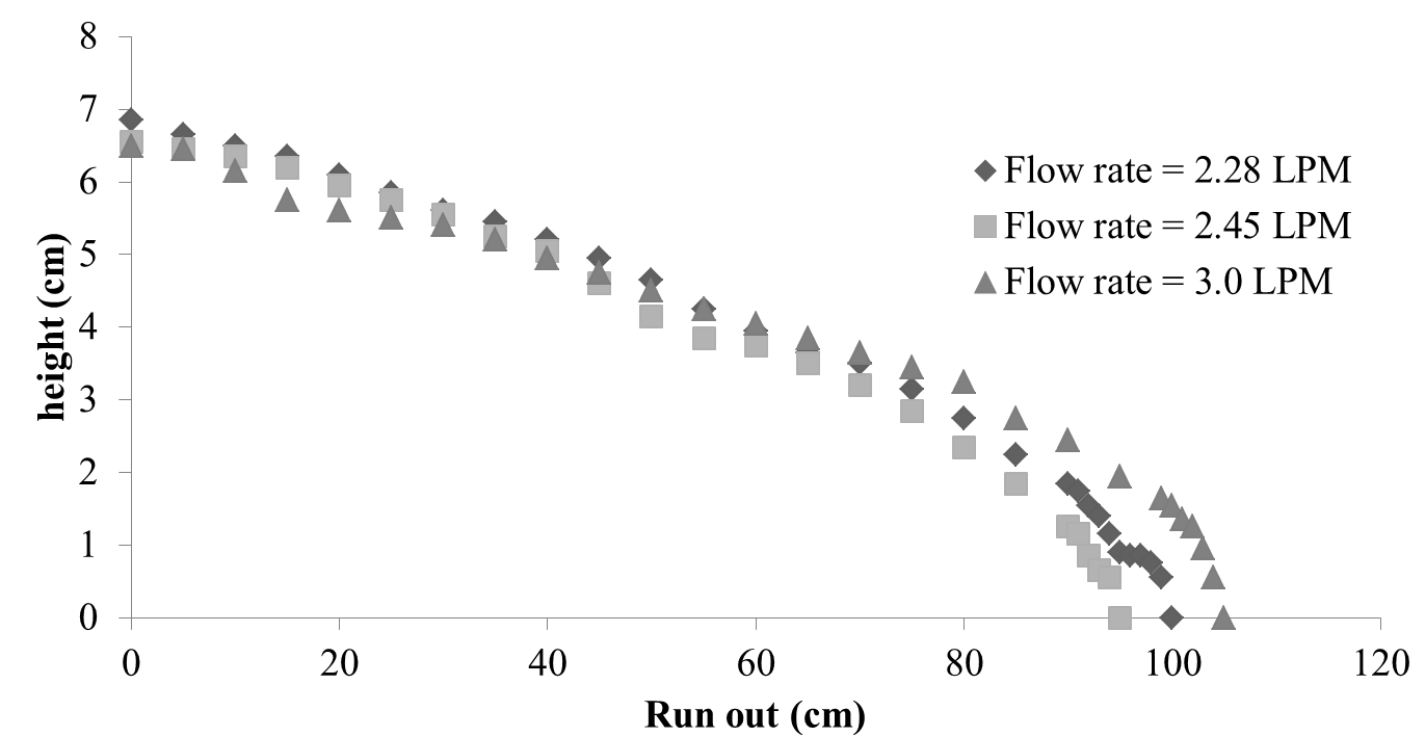

Figure 4.12: Final geometry of the Val D’Or tailings with different flow rates (at 52\% water content)

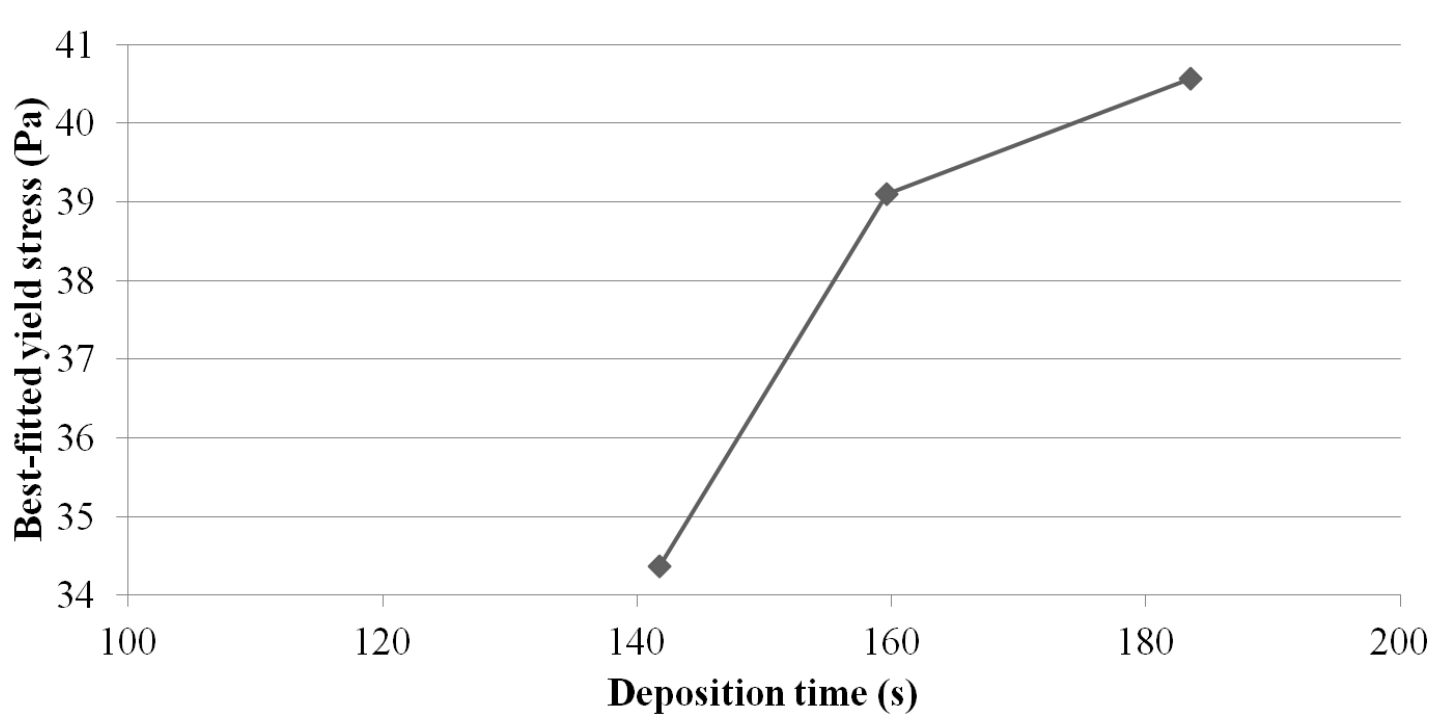

Figure 4.13: Best-fitted yield stress values versus deposition time for $52 \%$ water content.

The predicted and the measured final profiles for all the tests conducted using $52 \%$ water content provide similar results with the material with $53 \%$ water content. The predicted overall angle is steeper than the angle obtained from the experiment. Figure 4.14 presents the comparison of the final geometry for the smallest flow rate (2.28 LPM, test \#4). 


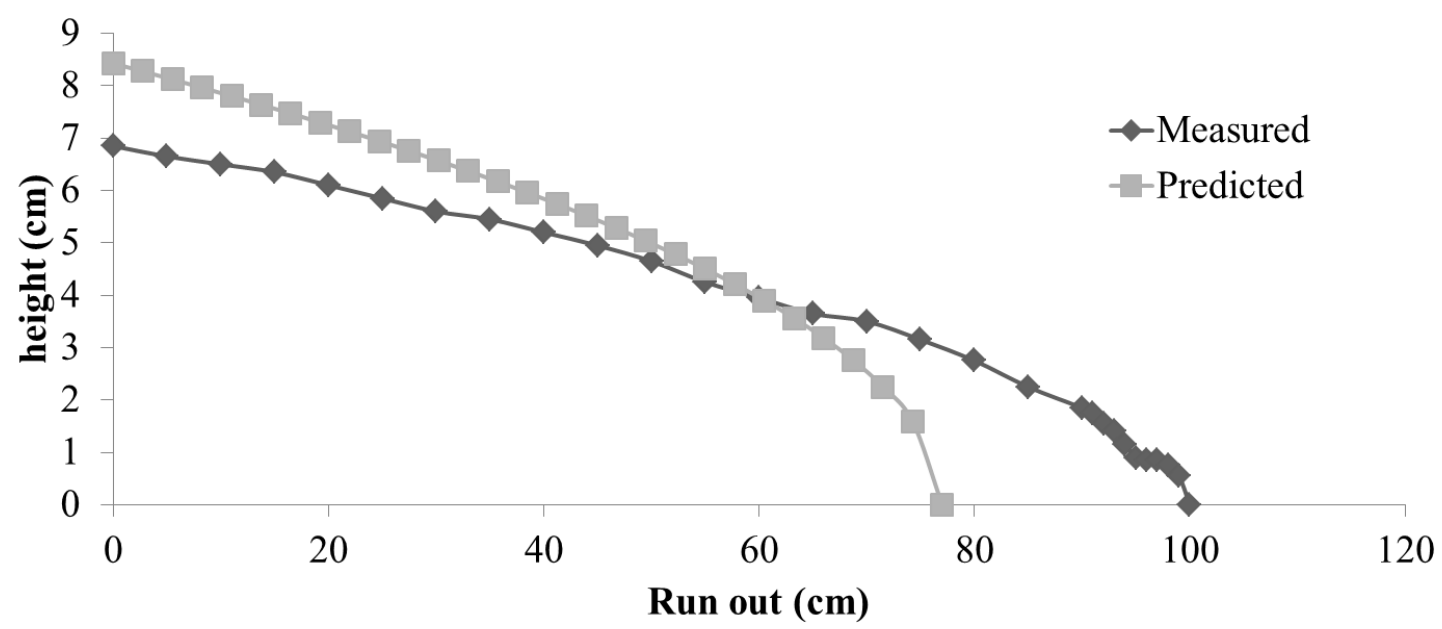

Figure 4.14: The comparison between the predicted and measured final profiles of the tailings (Val D'Or Batch \#1) at $52 \%$ water content (Flow rate $=2.28 \mathrm{LPM}, \tau_{\mathrm{y}}=80 \mathrm{~Pa}$ ).

The final division of the flume test with Val D'Or gold tailings is the material with $55 \%$ gravimetric water content. The summary of the tests conducted with this material is presented below.

\begin{tabular}{|c|c|c|c|}
\hline Test \# & Flow rate $(\mathrm{LPM})$ & Yield stress $(\mathrm{Pa})$ & Angle \\
\hline 6 & 3.45 & 50 & 2.6 \\
\hline 7 & 2.75 & 56 & 2.7 \\
\hline 9 & 2.45 & 55 & 3.7 \\
\hline
\end{tabular}

Table 4.6: Summary of flume test conducted with material at 55\% gravimetric water content.

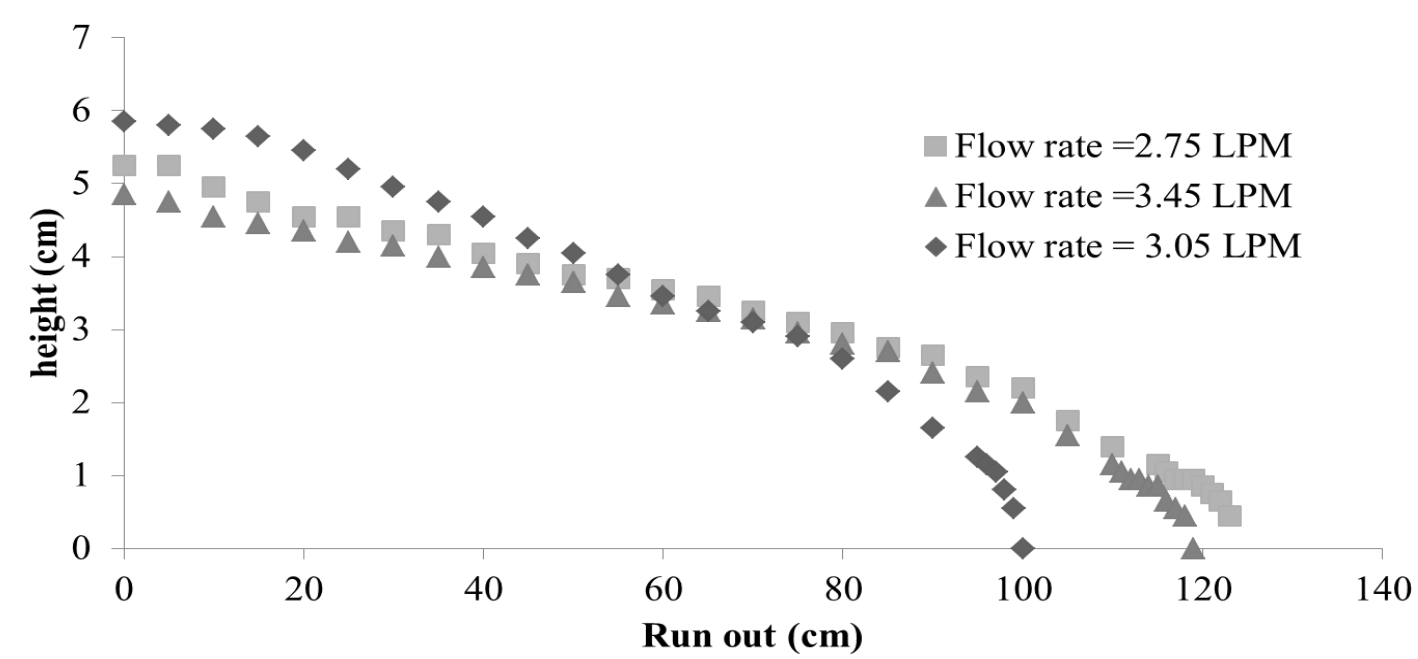

Figure 4.15: Final profiles of the tests summarized in Table 4.6 with $55 \%$ water content. 
The overall angles of the stacks are smaller compared to the previous tests which are expected since the water content is higher and the corresponding yield stress values are lower. However, similar to the previous test cases, the overall angles of final profile of the tailings are smaller compared to the predicted geometry. Figure 4.16 demonstrated the difference in the predicted and measured geometry of the tailings with 2.75 LPM deposition rate (Test \#7).

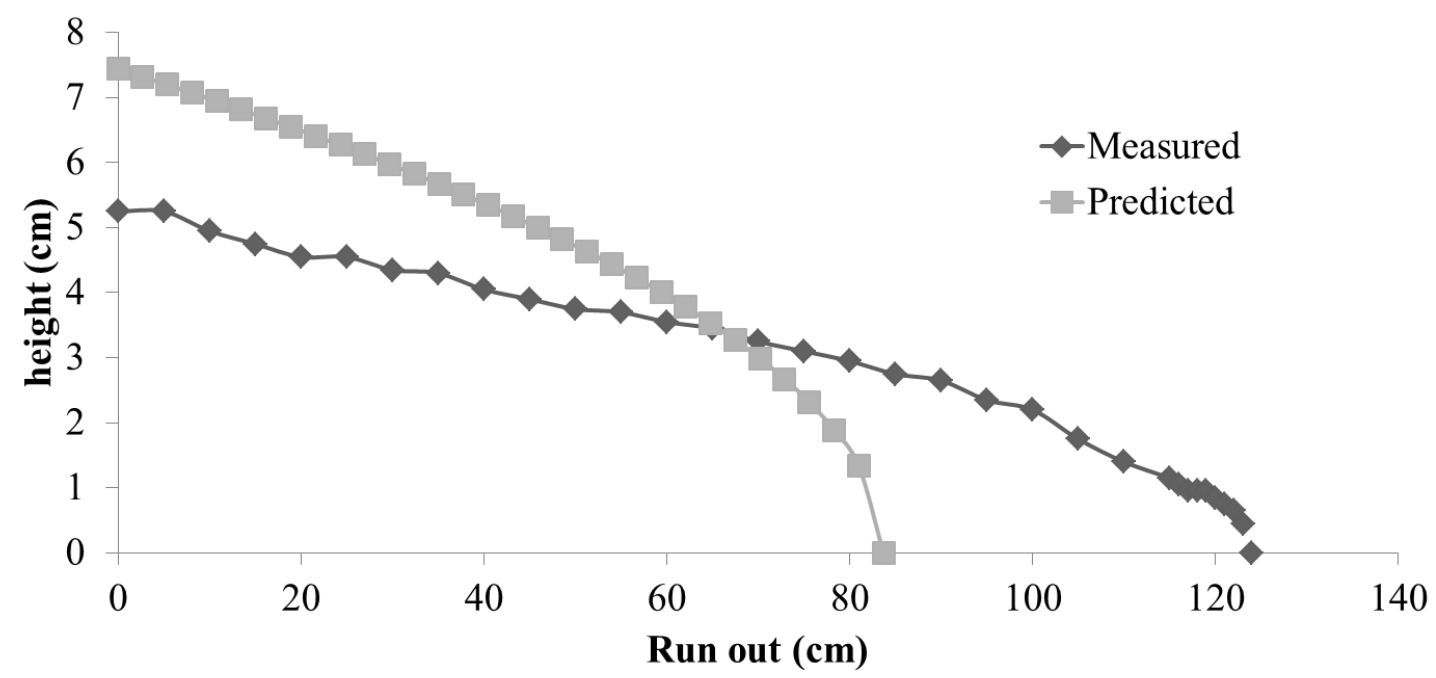

Figure 4.16: The comparison of predicted and measured final geometry of material with $55 \%$ gravimetric water content and 2.75 LPM deposition rate.

The only exception is the flume tests conducted with Val D'Or tailings is the test case \#10 which has relatively less water content (45\%) and the yield stress values determined from the slump tests is $103 \mathrm{~Pa}$. The predicted final geometry and the measured profile still demonstrated variations in terms of the heights along the run out distance but compared to the previous tests cases, it still gives a better correlation. 


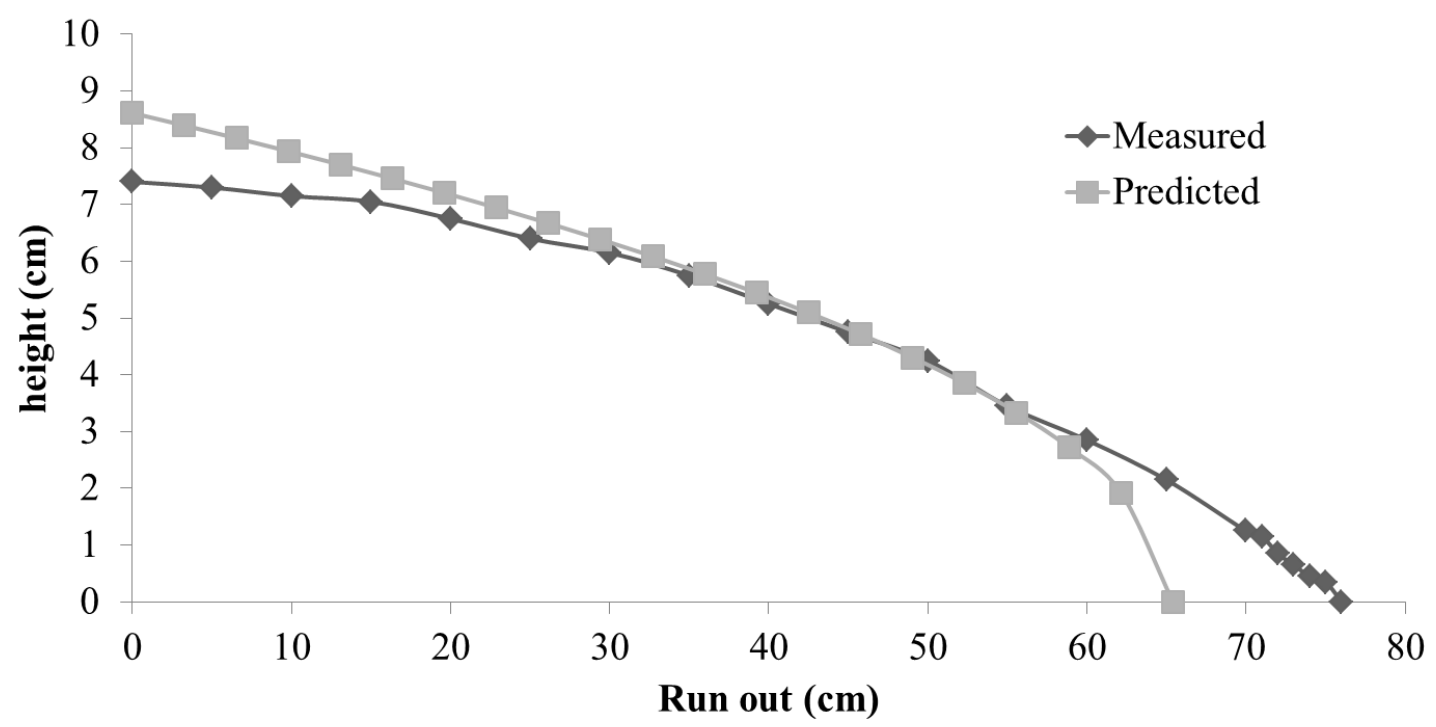

Figure 4.17: The comparison of predicted and measured final geometry of material with $44.5 \%$ gravimetric water content and 1.62 LPM deposition rate.

Since none of the tests gives good agreement with the theory, best fitted yield stress values are predicted using the Lubrication Theory using the run out distance and the initial height of the tailings determined from the flume test. The following table summarizes the yield stress values obtained from slump tests and the predicted yield stress values from the LT equation 3.3.

\begin{tabular}{|c|c|c|c|c|}
\hline Test \# & $\begin{array}{c}\text { Water content } \\
(\%)\end{array}$ & $\begin{array}{c}\text { Deposition rate } \\
\text { (LPM) }\end{array}$ & $\begin{array}{c}\text { Yield stress values } \\
\text { acquired from } \\
\text { Slump Test (Pa) }\end{array}$ & $\begin{array}{c}\text { Best fitted yield } \\
\text { stress values (Pa) }\end{array}$ \\
\hline 1 & 53 & 1.58 & 66 & 22 \\
\hline 2 & 52 & 3.0 & 79 & 34 \\
\hline 3 & 53 & 2.25 & 63 & 46 \\
\hline 4 & 52 & 2.28 & 80 & 41 \\
\hline 5 & 53 & 3.3 & 61 & 28 \\
\hline 6 & 55 & 3.45 & 50 & 16 \\
\hline 7 & 55 & 2.75 & 56 & 18 \\
\hline 8 & 54 & 2.4 & 62 & 28 \\
\hline 9 & 55 & 3.05 & 55 & 65 \\
\hline 10 & 45 & 1.62 & 103 & 39 \\
\hline 11 & 52 & 2.45 & 75 & \\
\hline
\end{tabular}

Table 4.7: The summary of best fitted yield stress values based on LT. 
As it can be observed in the table below, the fitted yield stress values are much smaller compared to the slump tests results.

\subsection{Pour Tests}

Total of three pour tests are conducted using Val D'Or tailings with different flow rates where the volume of each layer is same $(1.3 \mathrm{~L})$. The summary of the tests are presented in the following table.

\begin{tabular}{|c|c|c|}
\hline Pour test \# & Material (Val D'Or) & Flow rate (LPM) \\
\hline 1 & Batch 1 & 0.28 \\
\hline 2 & Batch 1 & 4.0 \\
\hline 3 & Batch 2 & 3.7 \\
\hline
\end{tabular}

Table 4.8: The summary of pour tests

\subsubsection{Val D'Or (Batch 1)}

The first two pour tests were conducted using the Val D'Or tailings collected from the first barrel and deposited with different flow rates. The rheological properties of tailings are presented in Table 4.1 and the yield stress values for different gravimetric water contents are demonstrated in Figure 4.3. This is the same material used in the flume tests. Each layer deposited in the test has a $1.3 \mathrm{~L}$ of volume and the layers are left to settle for 24 hours before depositing the new layer on top. It was observed during the experiments that, the height of the tailings are approximately decreases about 1-2 mm after completely settled.

Total of four layers were deposited in each tests where the initial water content of the material is approximately $55 \%$ which corresponds to $45 \mathrm{~Pa}$ of yield stress. After 24 hours 
of settling, the gravimetric water content dropped to $45 \%$; as a result the yield stress value increased to $170 \mathrm{~Pa}$.

The first test was deposited on the Plexiglas box with 0.28 LPM flow rate and the yield stress obtained from the slump test for each layer is presented in Table 4.9.

\begin{tabular}{|c|c|c|c|c|}
\hline Layer & $\begin{array}{c}\text { Initial water } \\
\text { content }(\%)\end{array}$ & $\begin{array}{c}\text { Final water content } \\
(\%)\end{array}$ & $\begin{array}{c}\text { Yield stress } \\
(\mathrm{Pa})\end{array}$ & $\begin{array}{c}\text { Density } \\
\left(\mathrm{kg} / \mathrm{m}^{3}\right)\end{array}$ \\
\hline 1 & 55 & 50 & 38 & 1706 \\
\hline 2 & 55 & 45 & 43 & 1709 \\
\hline 3 & 54 & 45 & 47 & 1714 \\
\hline 4 & 54 & 42 & 47 & 1722 \\
\hline
\end{tabular}

Table 4.9: Rheological properties of Val D'Or tailings for each layer for Pour test \#1

The final geometry of each layer is demonstrated in Figure 4.18; as it can observed in the figure the first two layers have fairly symmetric geometry; however as the thickness of the layers increases the geometry becomes axisymmetric. It was observed during the deposition of the last layers that the tailings have a tendency to flow in one direction but not on the same direction as the previous layer. 


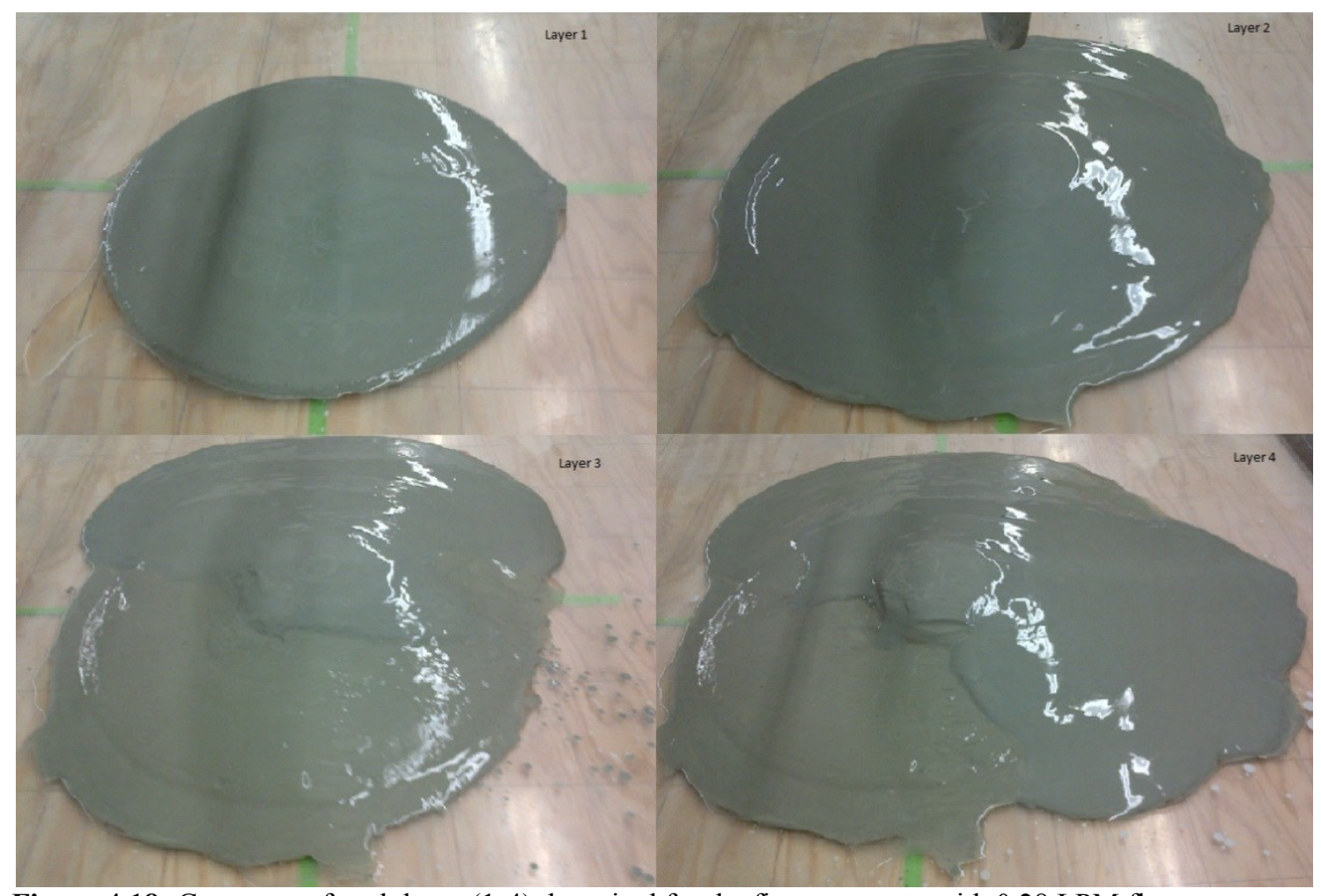

Figure 4.18: Geometry of each layer (1-4) deposited for the first pour tests with 0.28 LPM flow rate.

The prediction of the final geometry was determined by using Equations 3.3, for the first layer, and Equation 3.4 for the successive layers. The yield stress and density values used in LT equations are the same as the rheological properties presented in Table 4.9. The results showed similarities to the results from the flume tests where the predicted final angle is steeper than the angle calculated in the experiments. The difference between these geometries is presented in Figure $4.19 \& 4.20$. 


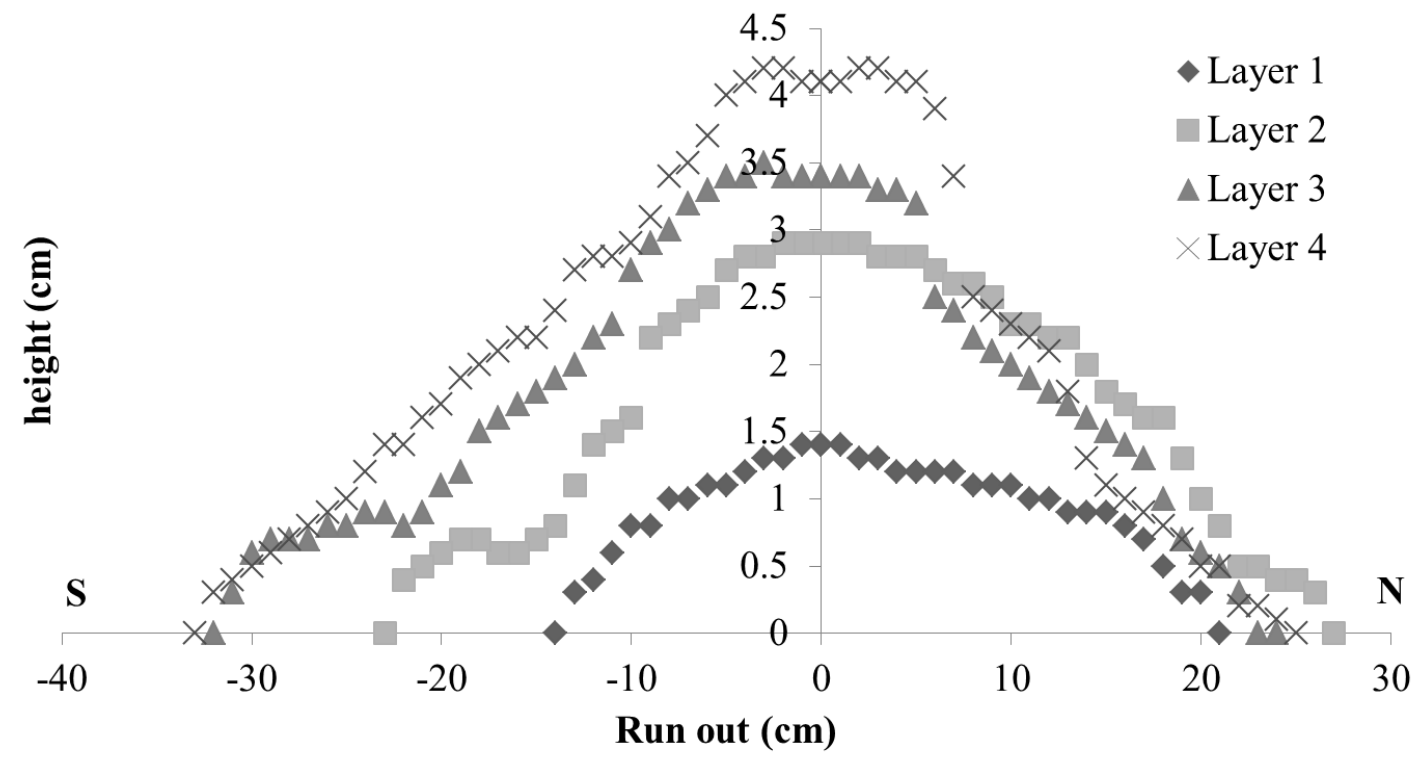

Figure 4.19: Final geometry measured from the experiment for Pour test \#1with 0.28 LPM flow rate (North - South direction)

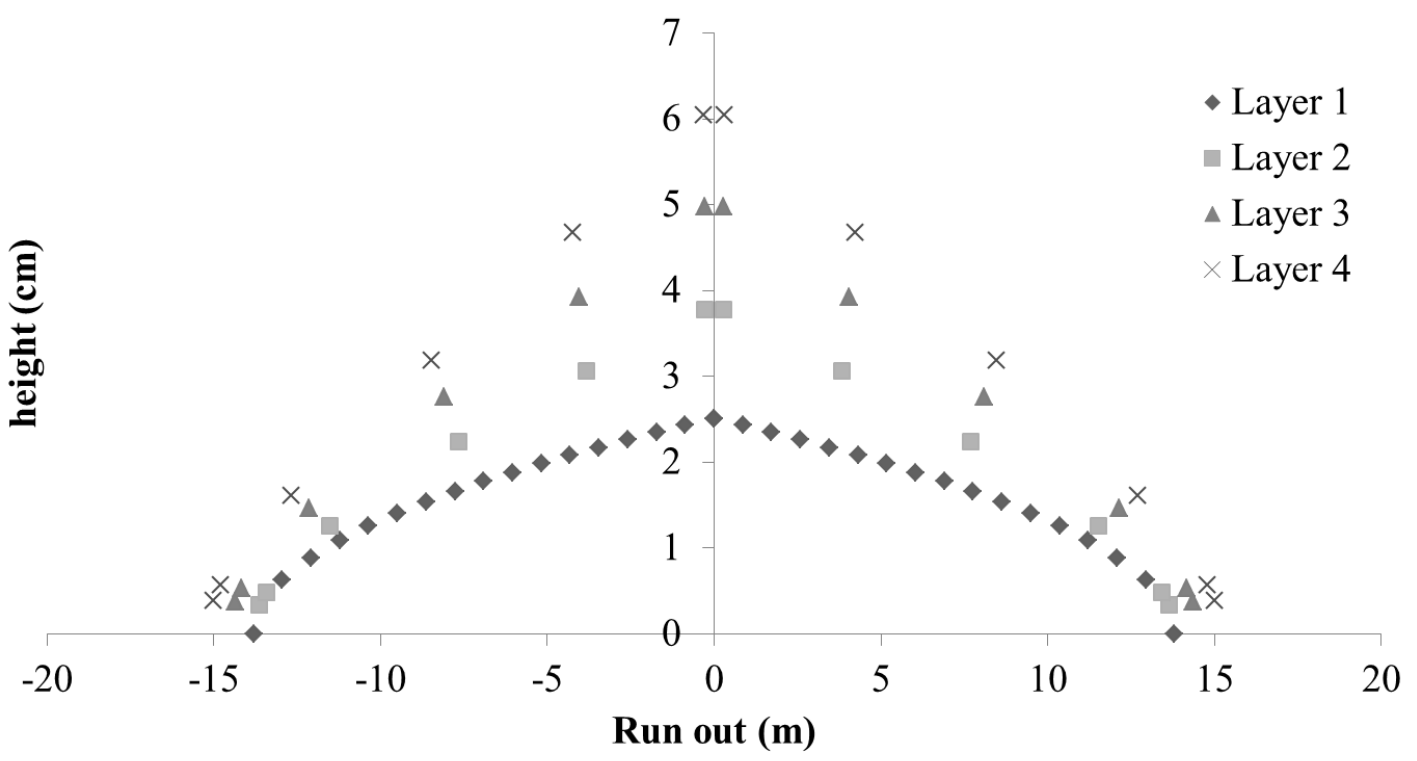

Figure 4.20: The final predicted geometry for Pour tests \# 1 with 0.28 LPM flow rate.

The results demonstrated that the prediction of the geometry and the measured data are not in good agreement. It should be reminded that the same problems were observed for the flume tests conducted using this material; these results are presented in the 
previous section. Best fitted yield stress values are calculated based on the Lubrication equations and the results are represented in Table 4.10 below. Also, the comparison between the final geometry predicted using the best-fitted yield stress values and the geometry obtained from experiment are demonstrated in the following figure.

\begin{tabular}{|c|c|c|}
\hline Layer & $\begin{array}{c}\text { Yield stress values from slump tests } \\
(\mathrm{Pa})\end{array}$ & $\begin{array}{c}\text { Best fitted Yield stress values } \\
(\mathrm{Pa})\end{array}$ \\
\hline 1 & 38 & 8 \\
\hline 2 & 43 & 25 \\
\hline 3 & 47 & 15 \\
\hline 4 & 47 & 15 \\
\hline
\end{tabular}

Table 4.10: Best fitted yield stress values for Pour test \#1.

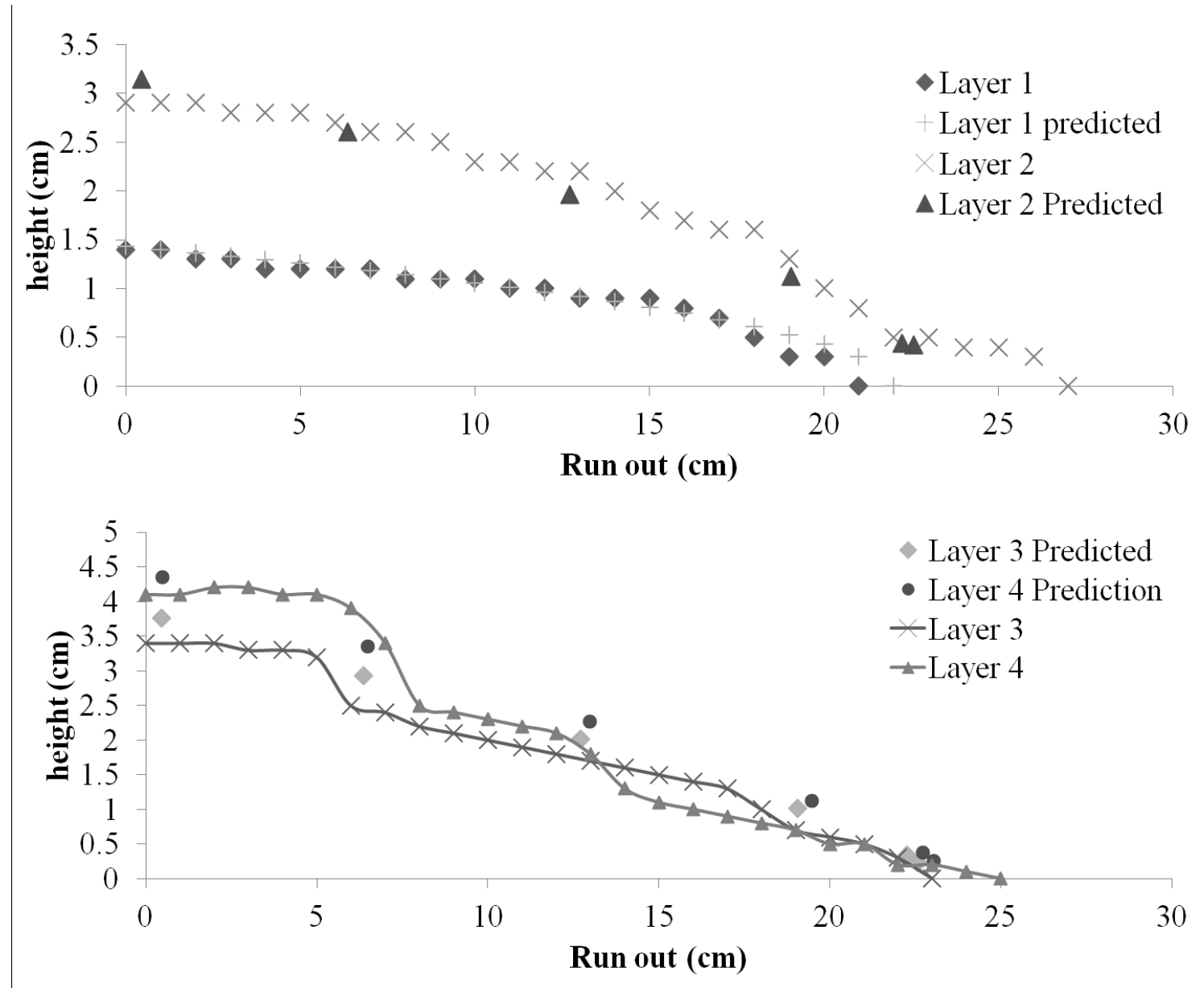

Figure 4.21: Comparison between the final geometry and predicted geometry using best-fitted yield stress values for Pour test (North direction) 
Another problem occurred during the experiment is the failure in the settled layers while depositing a new layer. The tailings should have enough strength to support the new deposited layers however the failures occurred in settled layers $2 \& 3$ in various directions. In Figure 4.22, the failures can observed at the middle of the layer at Northern and Western directions while another failure occurs at the toe of the stack in Eastern direction.
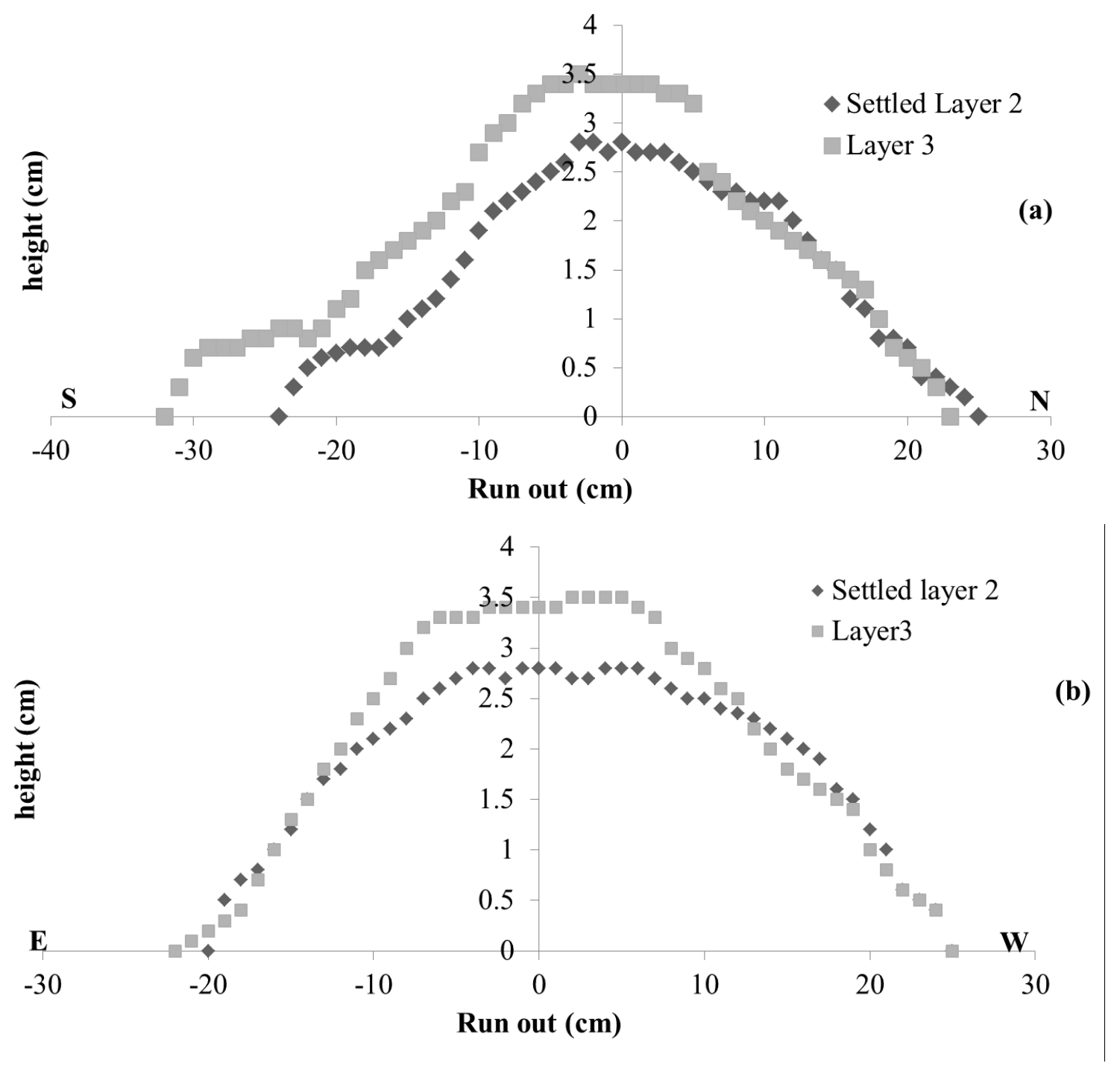

Figure 4.22: The failures in settled second layer in the directions of (a) North-South (b) West-East 
The same procedure is applied to the same batch of Val D'Or tailings with a higher flow rate in order to see its effect on the geometry. The rheological properties of each layer are displayed below.

\begin{tabular}{|c|c|c|c|c|}
\hline Layer & $\begin{array}{c}\text { Initial water } \\
\text { content }(\%)\end{array}$ & $\begin{array}{c}\text { Settled water } \\
\text { content }(\%)\end{array}$ & $\begin{array}{c}\text { Yield stress } \\
(\mathrm{Pa})\end{array}$ & $\begin{array}{c}\text { Density } \\
\left(\mathrm{kg} / \mathrm{m}^{3}\right)\end{array}$ \\
\hline 1 & 57 & 46 & 39 & 1755 \\
\hline 2 & 55 & 44 & 43 & 1725 \\
\hline 3 & 57 & 45 & 40 & 1772 \\
\hline 4 & 57 & 44 & 35 & 1758 \\
\hline
\end{tabular}

Table 4.11: Rheological properties of each Val D’Or tailings layer in Pour test \#2.

The initial water contents for the layers show some variance, the range is between 55 to $57 \%$. However, the settled water content is approximately $45 \%$ regardless of the initial water content. The flow rate of the test is $4.0 \mathrm{LPM}$ and the volume for each layer is $1.3 \mathrm{~L}$.

The final geometry has a smaller overall angle compared to the first test due to the velocity of the fluid. The velocity of the flow also affected the run out distances and the depth of the tailings layer. Similar to the first pour test, the first two layers has a fairly symmetric geometry and the flow changed directions in the last two layers. The final topography measured during the experiment and the predicted geometry based on LT equations are presented in the following figures. However, it can be observed that the predicted geometry has a steeper slope. In other words, for the measured yield stress values the tailings should demonstrate higher strength. It should be noted again that, this material is only collected from the top of the barrel, it contains mostly finer particles. 


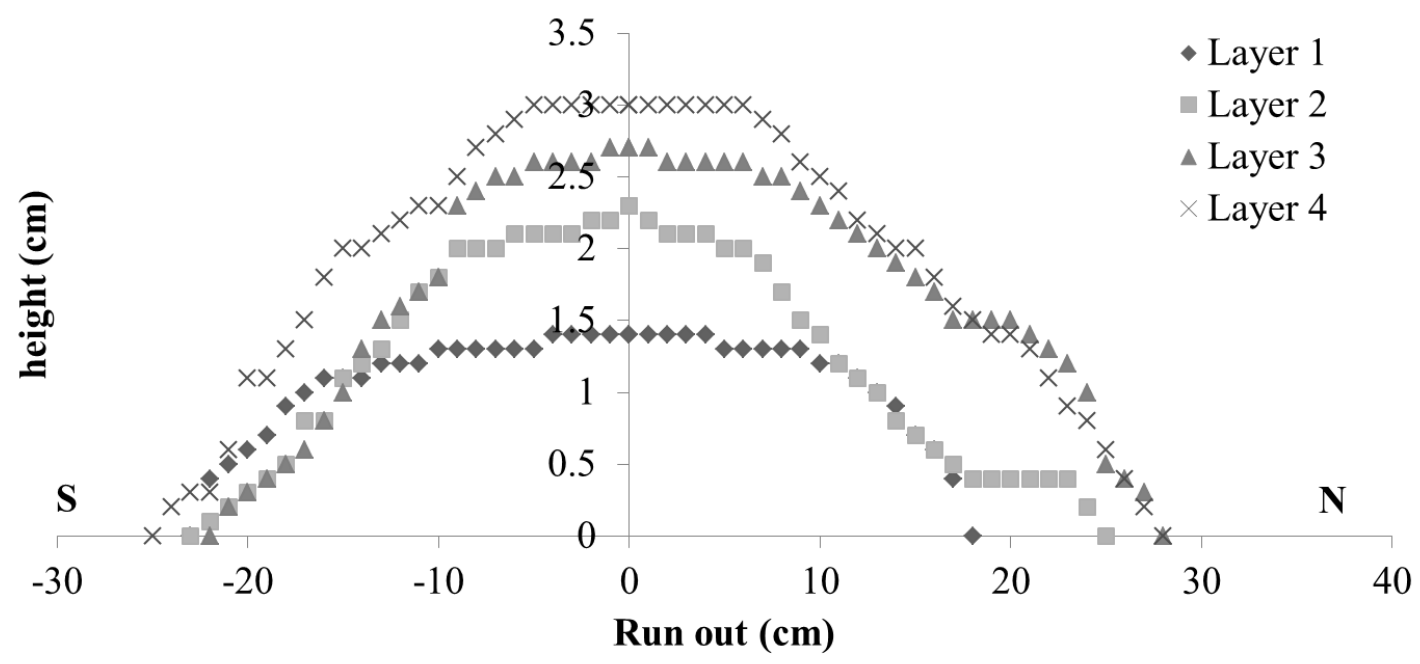

Figure 4.23: The measured final geometry for Pour test \#2 (North- South direction)

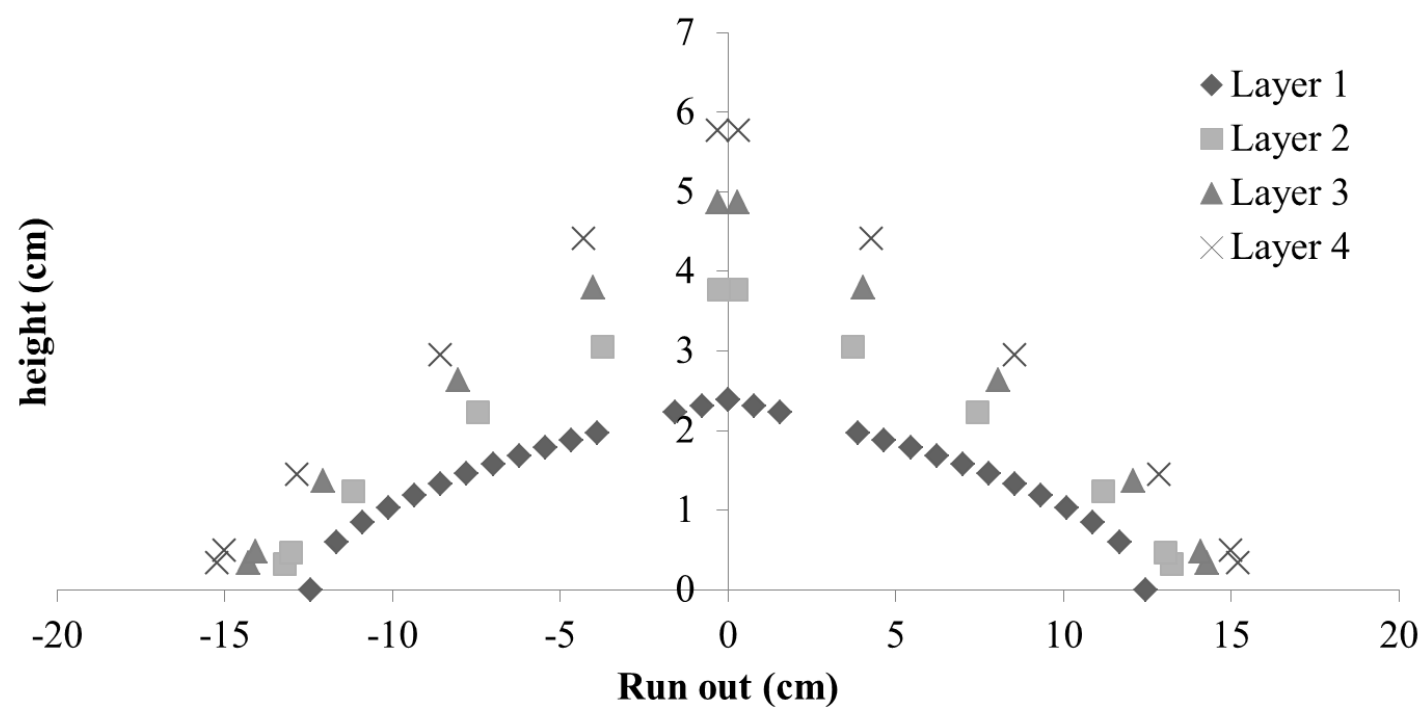

Figure 4.24: The predicted final geometry based on Lubrication equations for pour test \#2.

The same failure problem is also observed in the settled layers for this test as well. In the previous pour test, the failure didn't occur in the first settled layer; however, the each settled layer failed to carry the new deposited layer in this test in various directions. 

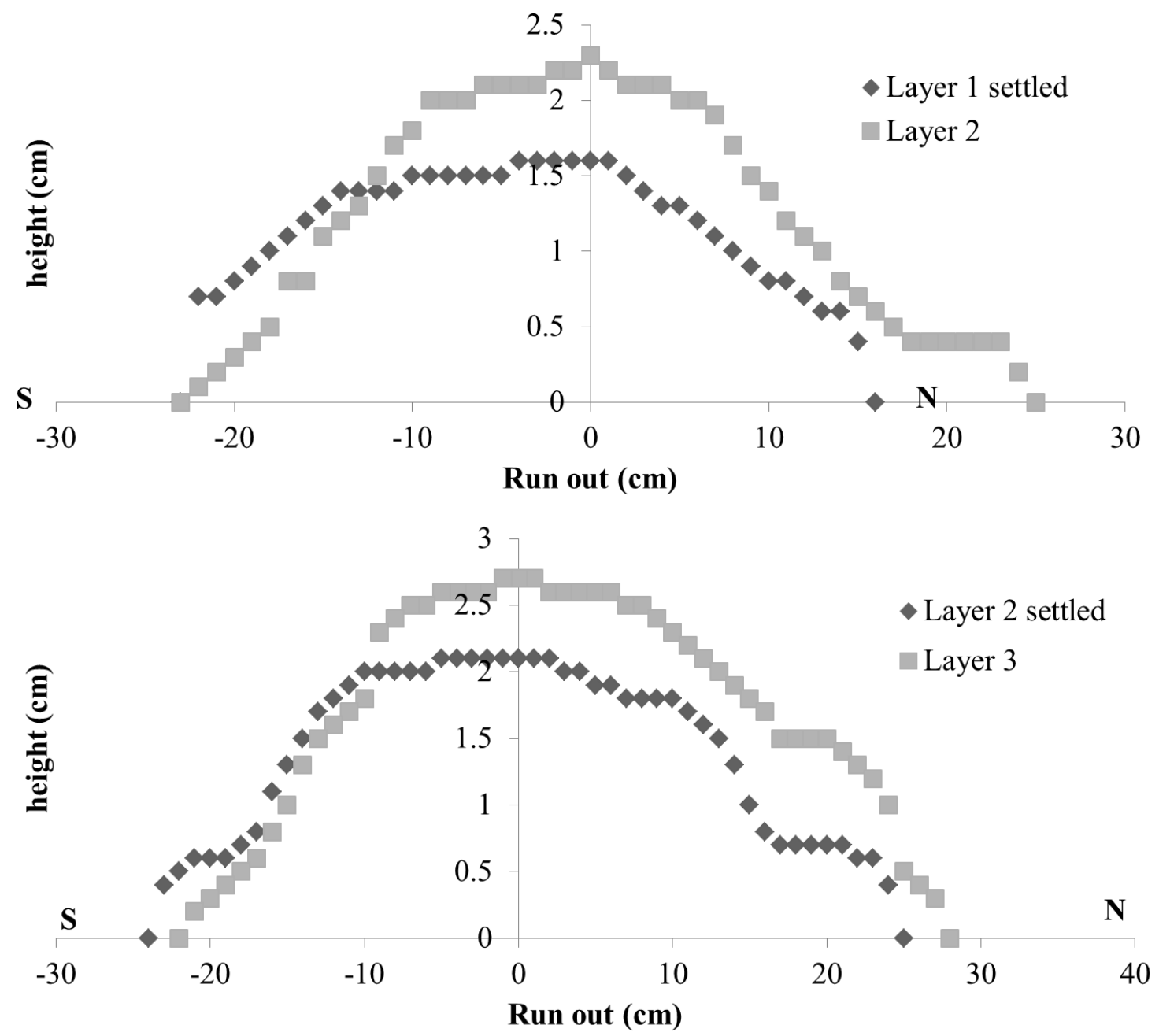

Figure 4.25: The failure in settled layers in Pour tests \#2 (North-South Direction).

In conclusion, the material didn't gain enough strength for both of the pour tests regardless of the changes in flow rates and geometry. In order to achieve successful results, a new barrel of Val D'Or tailings are opened and the barrel is mixed thoroughly. Hydrometer test was performed to the sample tailings collected from the top, middle and the bottom section of the barrel make sure the particle size distribution is uniform. The excessive water on top of the barrel is removed until the final yield stress values obtained 
from the slump tests are approximately $45 \mathrm{~Pa}$ which corresponds to $41 \%$ of gravimetric water content.

\subsubsection{Val D'Or (Batch 2)}

The final pour test was performed using the material from the second barrel. The rheological properties of tailings are presented in Table 4.1 and the yield stress values for different gravimetric water contents are demonstrated in Figure 4.3.

Total of five layers are deposited where each layer has a $1.3 \mathrm{~L}$ of volume and the layers are left to settle for 24 hours before depositing the new layer on top and the stack is covered with a plastic sheet to minimize the evaporation. The particles completely stopped settling after 9.5 hours. The rheological properties of each layer are displayed in Table 4.12 below.

\begin{tabular}{|c|c|c|c|c|}
\hline Layers & $\begin{array}{c}\text { Initial water content } \\
(\%)\end{array}$ & $\begin{array}{c}\text { Final water content } \\
(\%)\end{array}$ & $\begin{array}{c}\text { Yield stress } \\
(\mathrm{Pa})\end{array}$ & $\begin{array}{c}\text { Density } \\
\left(\mathrm{kg} / \mathrm{m}^{3}\right)\end{array}$ \\
\hline 1 & 42 & 38 & 37 & 1855 \\
\hline 2 & 41 & 35 & 46 & 1841 \\
\hline 3 & 40 & 35 & 56 & 1855 \\
\hline 4 & 40 & 34 & 55 & 1821 \\
\hline 5 & 41 & 35 & 47 & 1873 \\
\hline
\end{tabular}

Table 4.12: The rheological properties of each layer for Pour test \#3.

The deposition rate of the last pour test is 3.7 LPM where the initial gravimetric water content of the tailings is $41 \%$. The material is settled completely to $35 \%$ gravimetric content; therefore the settled material has strength higher than $200 \mathrm{~Pa}$ (Figure 4.3). The measured topographies in different directions are represented in the following figure. 

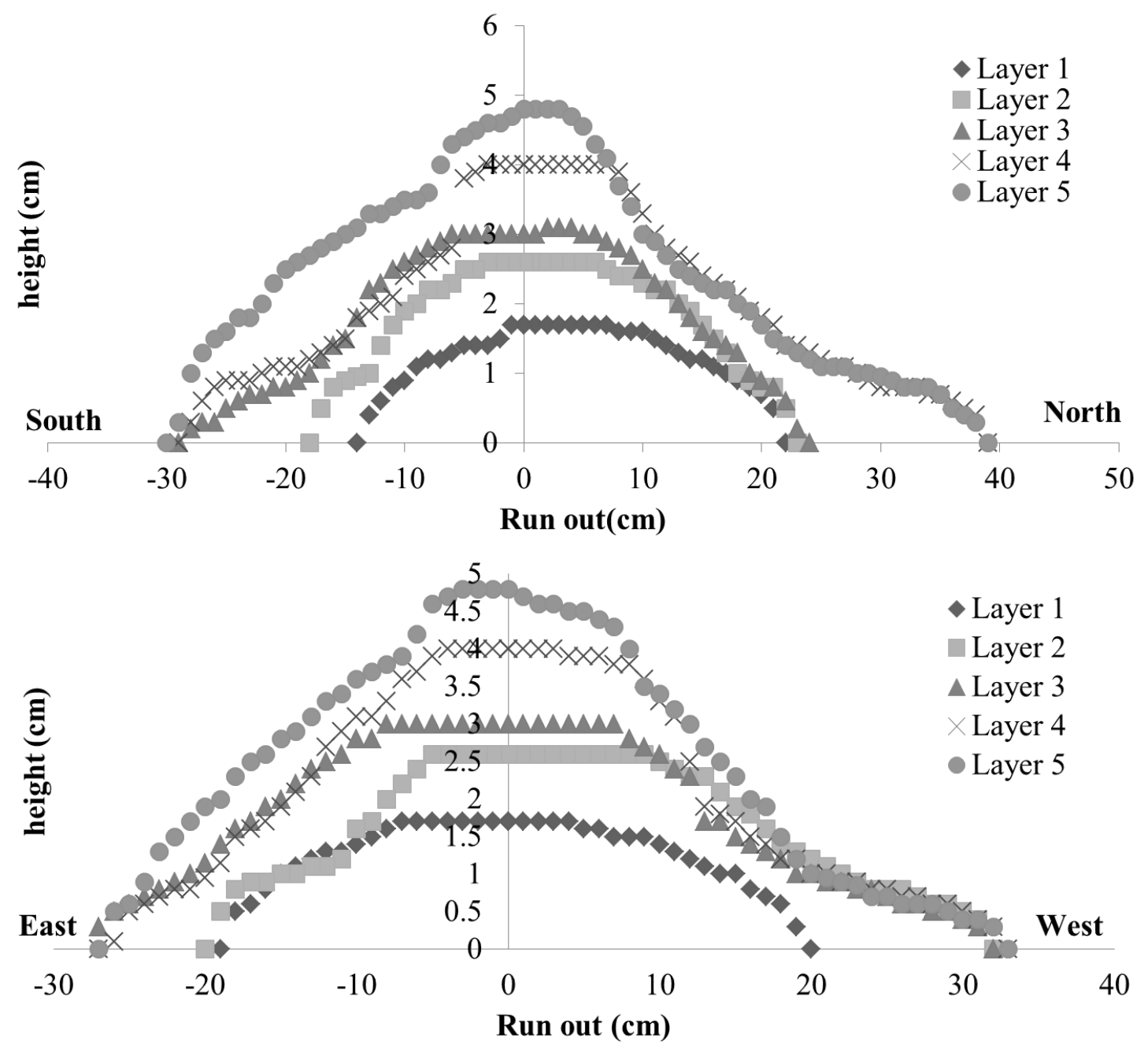

Figure 4.26: The measured topography of Val D'Or tailings of pour test \#3 in (a)North-South and WestEast directions (3.7 LPM deposition rate and 1.3 L of volume)

The geometry of the first few layers exhibit axis-symmetric profiles but as the layer thickness increased, the tailings started to flow in different directions for each layer creating an asymmetrical final geometry (Figure 4.26). Unlike previous tests cases, there were no failures observed in the settled layers. The predicted geometry of the tailings demonstrated that the final topography of the Val D'Or tailings have a steeper angle where the selected yield stress in the calculations are the same as the measured yield 
stress values from the slump tests (Figure 4.28). The best fitted yield stress values are calculated using Equations 3.3 and 3.4 and presented in Table 4.13.

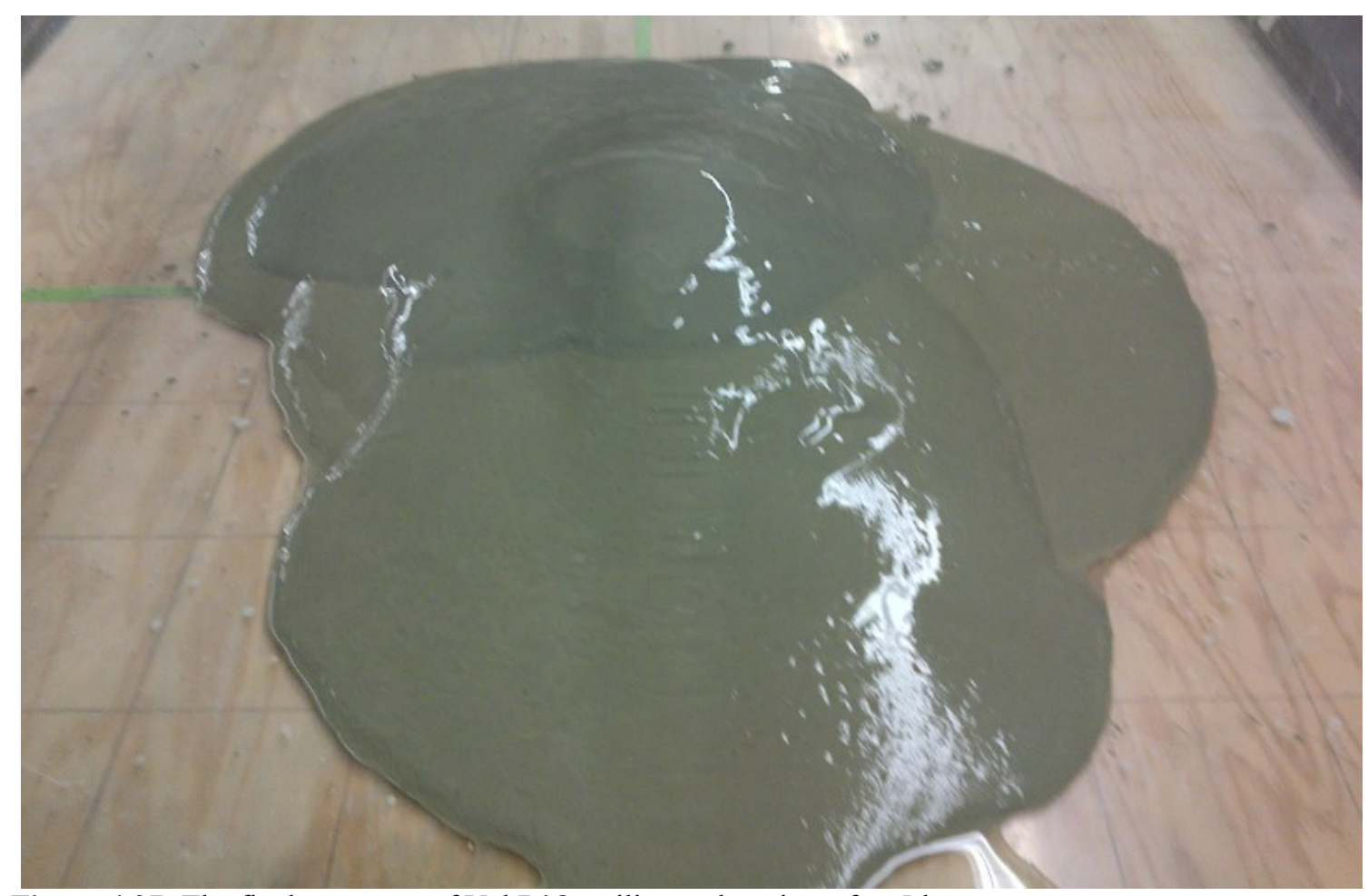

Figure 4.27: The final geometry of Val D'Or tailings, plan view after 5 layers. 


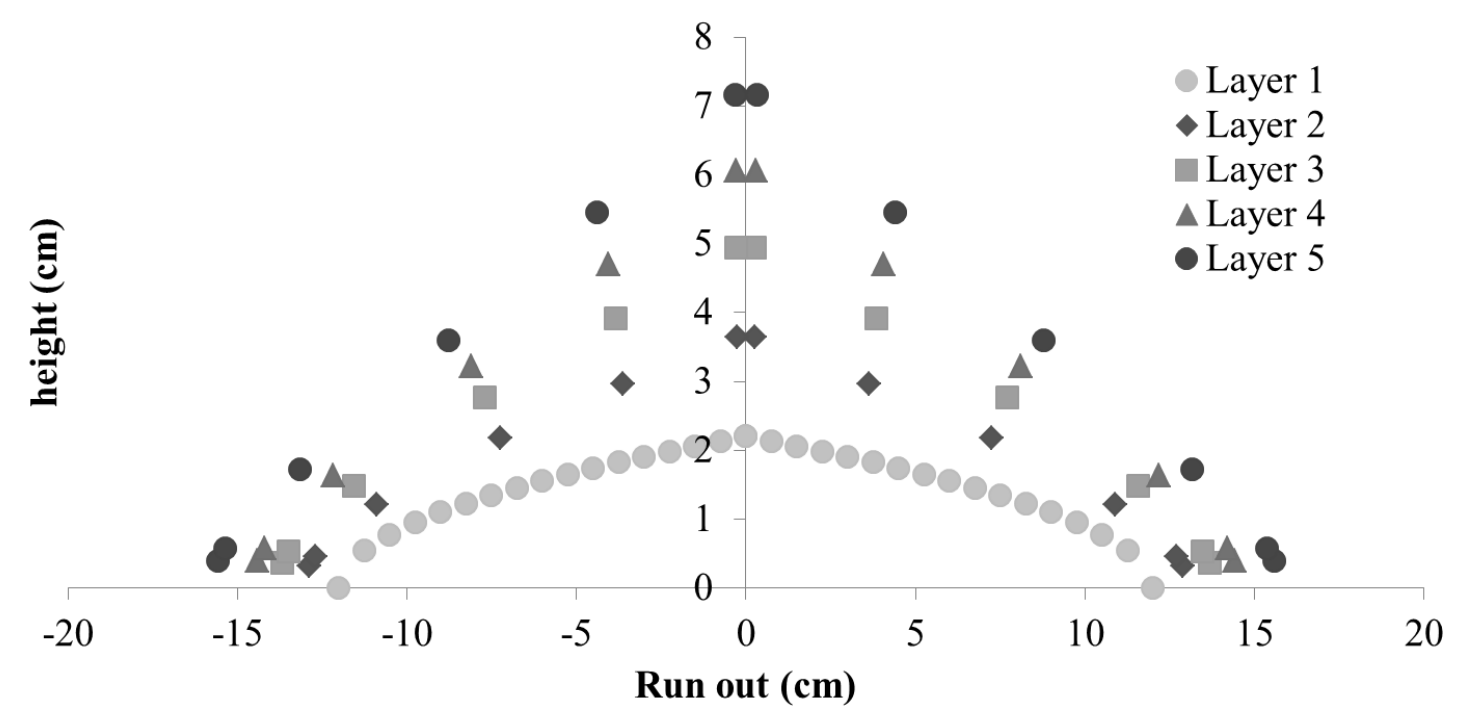

Figure 4.28: The predicted topography of Val D'Or tailings.

\begin{tabular}{|c|c|c|}
\hline Layers & $\begin{array}{c}\text { Yield stress values from slump } \\
\text { test }(\mathrm{Pa})\end{array}$ & $\begin{array}{c}\text { Best-fitted yield stress values } \\
(\mathrm{Pa})\end{array}$ \\
\hline 1 & 37 & 20 \\
\hline 2 & 46 & 20 \\
\hline 3 & 56 & 13 \\
\hline 4 & 55 & 13 \\
\hline 5 & 47 & 13 \\
\hline
\end{tabular}

Table 4.13: Best-fitted yield stress results calculated using Equation 3.3 and 3.4.

As it was mentioned previously, the distribution of each layer is not uniform after the second layer. Tailings tend to flow in one direction on each deposition. Best-fitted yield stress values demonstrated in Table 4.13 was calculated based on the data measured on the South direction. As it can observed, tailings flow through south-east direction during the deposition of third layer. As a result, increase in the run out distance is very small and predicted yield stresses were lower compared to the first two layers. The comparison between topography obtained by using best-fitted yield stress and the measured final geometry is presented in Figure 4.29. 


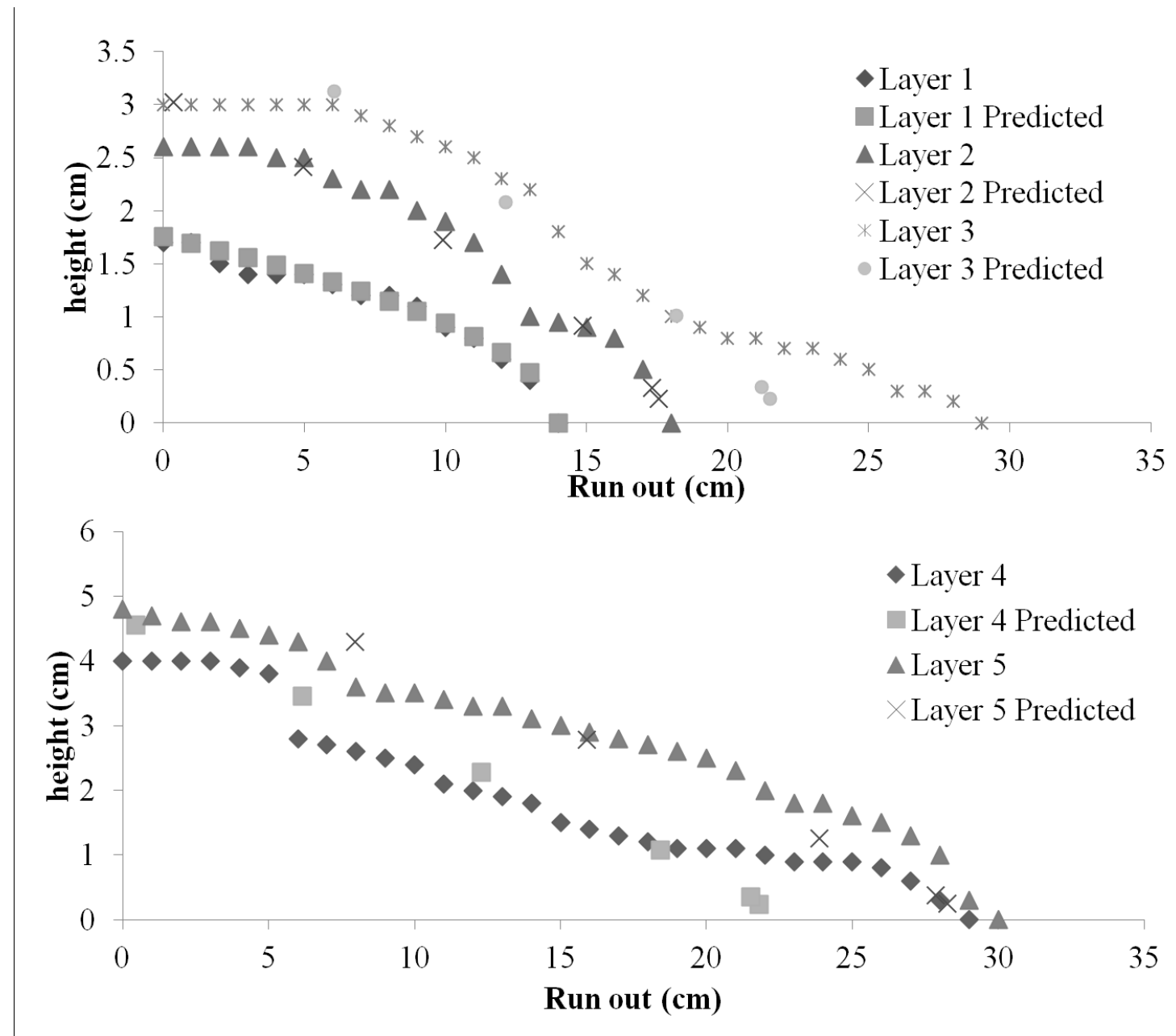

Figure 4.29: Predicted topography using best-fitted yield stress values (South direction)

Mizani, et al. (2013) obtained similar results from pour tests conducted using Bulyanhulu tailings; the topography of the first few layers are fairly symmetric and as the layer number increases the flow regime changes and exhibit a convex profile. Therefore, angle closer to deposition point is steeper than the angle near the edge of the footprint. Figure 4.26 clearly demonstrates the change in the flow profile after the deposition of the third layer. 
In conclusion, the flume tests conducted using Bulyanhulu tailings demonstrated that the final topography is affected by the deposition flow rates. For higher flow rates, estimated yield stress values are closer to the values obtained from slump test. An increase in the deposition time results in an increase in the yield stress values due to the effect of settling; similar results are also found in Mizani, et al. (2013). In addition, for flow rates higher than 2.5 LPM the predicted final geometries gives better correlation with the Lubrication Theory.

The effect of the initial water content on the overall slope angle and run out distance is presented with the flume experiments using Val D'Or tailings which are also studied in Henriquez and Simms (2009) for Bulyanhulu tailings. It was found that the lowest water content has the shortest run out distance and highest overall slope angle; agreeing with their findings. However, unlike their results Lubrication Theory did not agree well with the experimental data since the Val D'Or tailings used in the flume tests are dominated by finer particles.

The limitations of LT evident to this chapter and previous work showed that a more fundamental approach is needed to analyze even small scale bench tests. The next chapter investigates the applicability of one such method: CFD modeling using SPH. 


\section{Chapter: Numerical Analysis and Results}

\subsection{Implementation and Formulation in SPHYSICS}

Smoothed Particle Hydrodynamics (SPH) method based SPHYSICS program was chosen for the implementation of the experimental tests in this research. Originally developed for Newtonian fluids, some modifications have to be considered in the program for it to be applicable for non-Newtonian fluids. The theoretical background of the method is explained in Chapter 2. The implementation of the method including time stepping algorithm and boundary conditions are discussed in the following sections. The modifications on the rheological model and consequently governing equations are reviewed. Finally, the implementation of the model to single and multilayer depositions of high density tailings is described.

\subsubsection{Rheological Model}

Several researchers studied the behavior of non-Newtonian fluids using SPH method. Ellero, et al. (2002) reproduce viscoelastic model through a Maxwell model using SPH and Shao and Lo (2003) simulated a non-Newtonian free surface problem with the use of Cross model as the rheological model. Hosseini, et al. (2007) proposed a three-step algorithm for the simulation of non-Newtonian fluid flow. Capone (2009) used SPH method for simulating non-Newtonian landslide deformation and its interaction with water. 
The fluid in our model is treated as a Bingham plastic where the fluid at rest is capable of resisting any shear below the yield. The constitutive equation of generalized Newtonian fluid has to be referred to define Bingham fluid. The shear stress $\tau$ is a function of shear rate tensor D (Hosseini, et al. 2007).

$$
\mathbf{D}=\frac{1}{2}\left(\nabla \mathbf{v}+\nabla \mathbf{v}^{\mathrm{T}}\right)
$$

The viscosity of the fluid is dependent on the second invariant of shear strain rate, $|\mathbf{D}|$ is calculated as;

$$
|\mathbf{D}|=\sqrt{\sum_{i j} \mathbf{D}_{i j} \mathbf{D}_{i j}}
$$

The constitutive relationship for Newtonian fluids is defined as (Vola, et al. 2004);

$$
\tau=\mu(|\mathbf{D}|) \mathbf{D}
$$

which demonstrates the relationship between the viscosity $\mu$ and shear strain $\mathbf{D}$. The Bingham plastic behavior suggests that there is no deformation below the critical yield stress. Above this level, the material behavior is similar to Newtonian fluid and the viscosity is dependent on the yield stress as well as the second invariant of the shear rate tensor. The 'bi-viscosity model' is proposed to define the Bingham plastic in the proposed model here (Beverly and Tanner 1992; Hosseini et al. 2007; Capone 2009). The main difference between the classical model and this model is that the viscosity is defined in the un-yielded region as well. Instead of defining no deformation below the critical zone, greater viscosity values are presented to the fluid. As a result, the transition between these two zones is smoother. The constitutive relationship can be defined as; 


$$
\begin{aligned}
& |\mathbf{D}| \leq \frac{\tau y}{2 \alpha \mu} \rightarrow \tau=2 \alpha \mu \mathbf{D} \\
& |\mathbf{D}|>\frac{\tau y}{2 \alpha \mu} \rightarrow \tau=\left(\frac{\tau y}{|\mathbf{D}|}+2 \mu\right) \mathbf{D}
\end{aligned}
$$

where $\tau_{\mathrm{y}}$ is the yield stress, $\mu$ is the viscosity, $\alpha$ represents the coefficient that gives a greater viscosity value in the un-sheared zone. $\mathbf{D}$ is the shear rate tensor and $|\mathbf{D}|$ is the second invariant of the shear strain rate.

In the literature, the most common $\alpha$ value is 100 (Hoseini et al, 2007; Beverly and Tanner 1992); however, this value depends on the characteristics of the non-Newtonian fluid simulated. The sensitivity of the model to the yield stress, viscosity and $\alpha$ value are tested and the results are compared with the experimental data acquired from Henriquez and Simms (2009). Total of $5500 \mathrm{~cm}^{3}$ of Bulyanhulu paste tailings are deposited on the reservoir section of the flume (particle positions are shown in Figure 5.12); then the gate is removed and the tailings flow. The yield stress value of the tailings selected for the model is $36 \mathrm{~Pa}$ where the viscosity is 0.65 Pa.s; both obtained from Henriquez and Simms (2009). The best fitted $\alpha$ value was found to be 40 and the sensitivity of the model to various inputs are presented below.

In order to determine the effect of inertia, the same test simulated (volume $5500 \mathrm{~cm}^{3}$ ) but with various heights; $0.1,0.2,0.3,0.4,0.8$ metres respectively and results are compared with Lubrication Theory. The results showed that predicted profiles have shorter flow distance and steeper angles. The change in the theoretical and numerical results started to distinguish where the initial height of the gate is higher than 0.4 metres (Figure 5.2). 

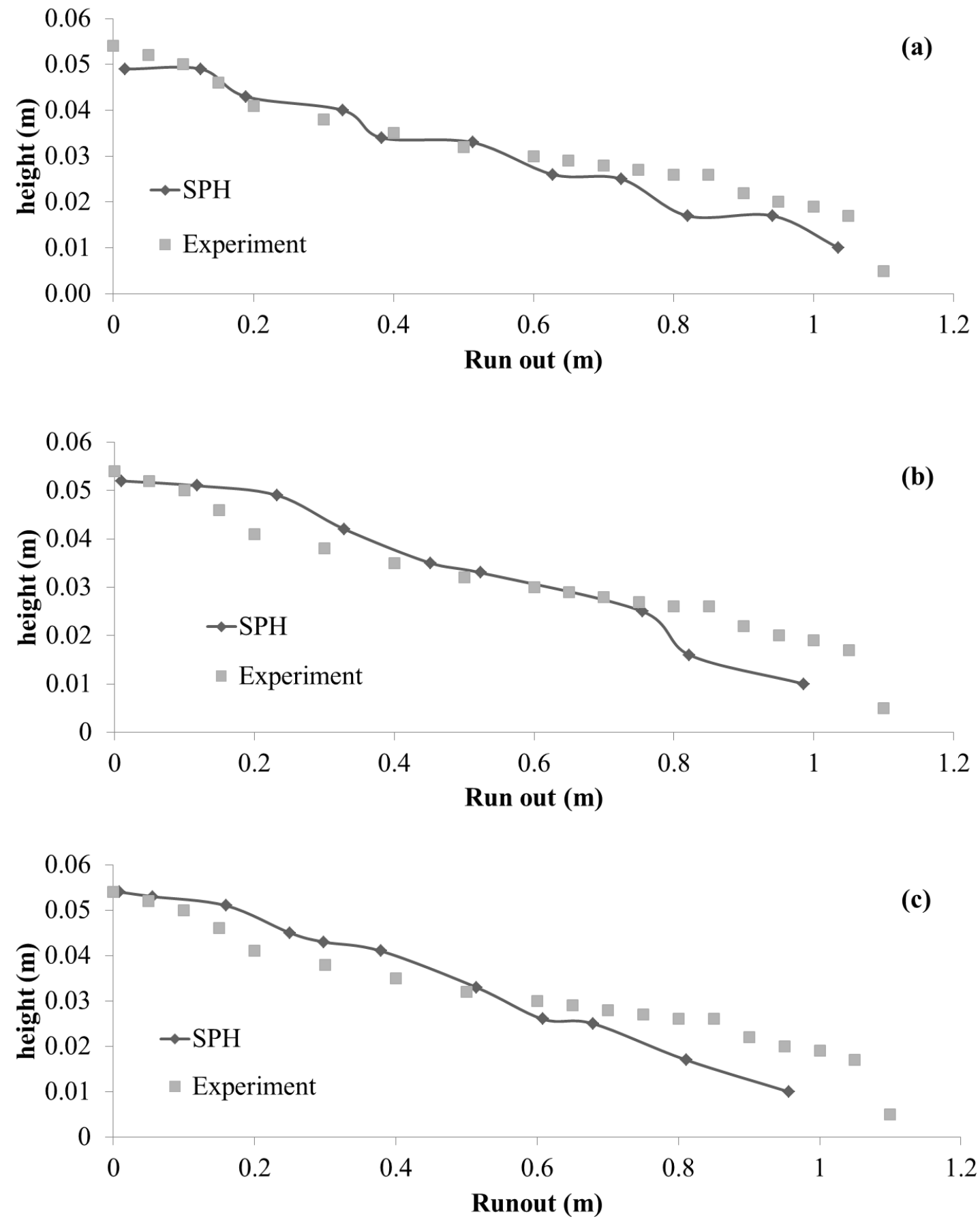


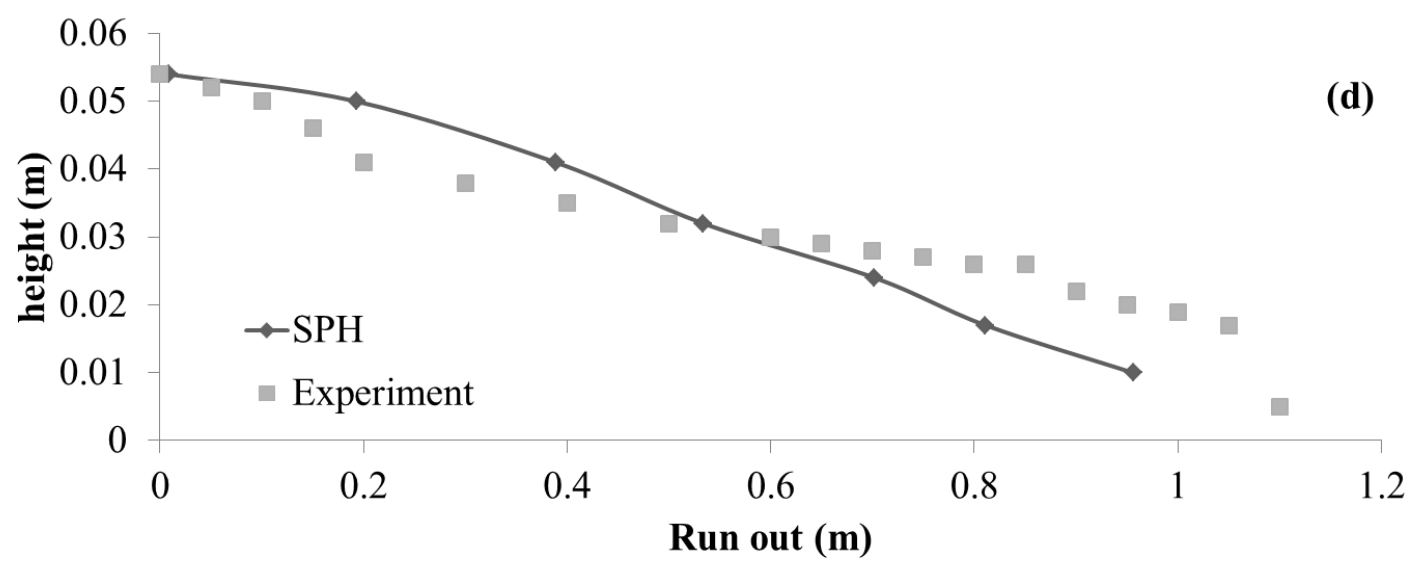

Figure 5.1: The sensitivity of the model to various inputs (a) $\tau_{\mathrm{y}}=36 \mathrm{~Pa} . \mu=0.65 \mathrm{~Pa} . \mathrm{s} \alpha=40$ (b) $\tau_{\mathrm{y}}=36 \mathrm{~Pa}$. $\mu=1.5$ Pa.s $\alpha=40$ (c) $\tau_{\mathrm{y}}=72$ Pa. $\mu=0.65$ Pa.s $\alpha=40$ (d) $\tau_{\mathrm{y}}=36$ Pa. $\mu=0.65$ Pa.s $\alpha=100$
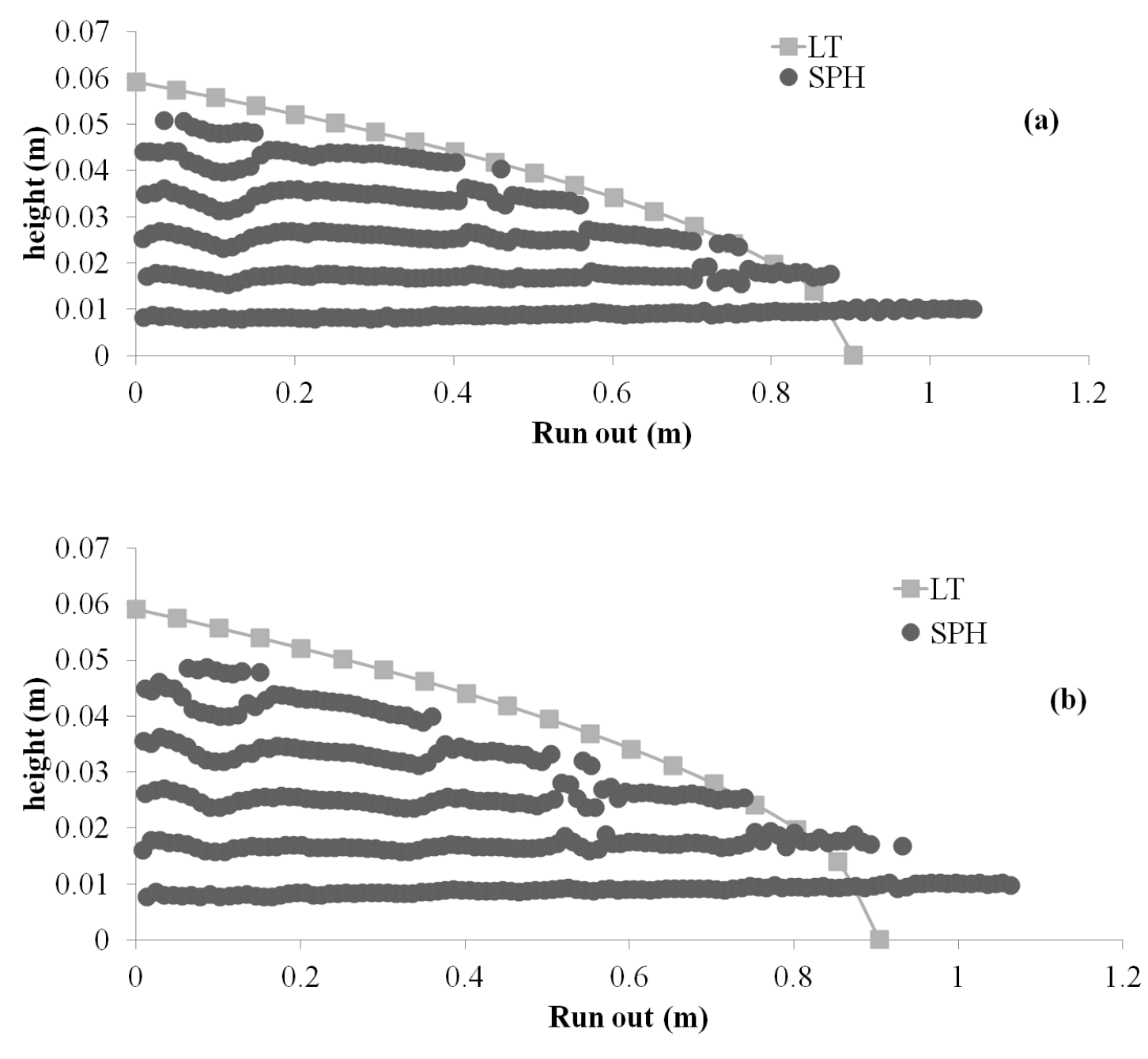

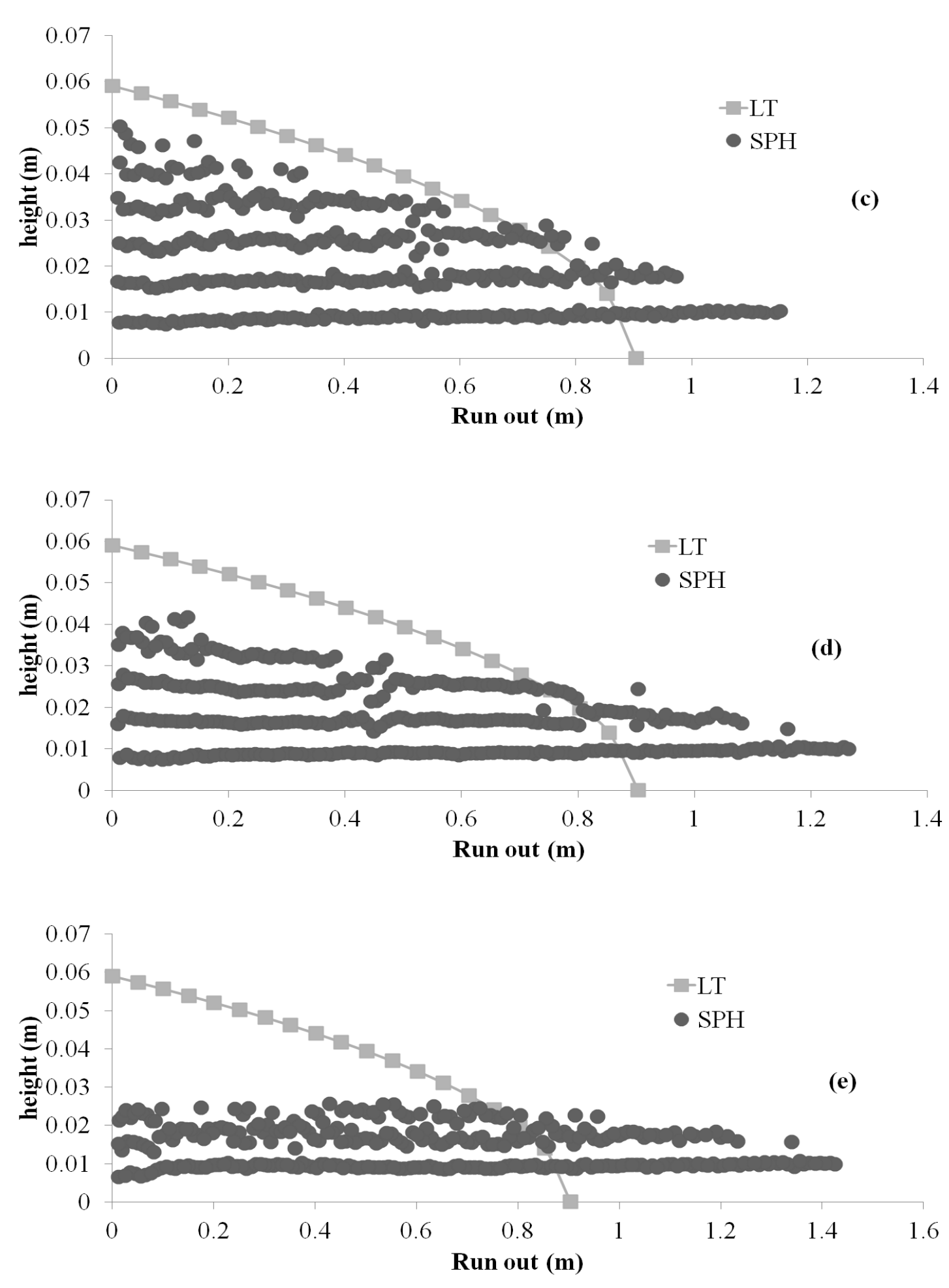

Figure 5.2: The effect of inertia demonstrated for different initial conditions for the same volume of tailings (a) h=0.1 m (b) $0.2 \mathrm{~m}$ (c) $0.3 \mathrm{~m}$ (d) $0.4 \mathrm{~m}$ (e) $0.8 \mathrm{~m}$. 
An effective viscosity term is added in the momentum equation to define the Bingham fluid (Capone, et al. 2010; Hosseini, et al. 2007).

$$
\frac{d \mathbf{v}_{a}}{d t}=-\sum_{b} m_{b}\left(\frac{P_{a}}{\rho_{a}{ }^{2}}+\frac{P_{b}}{\rho_{b}{ }^{2}}+\Pi_{a b} e f f\right) \nabla_{a} W_{a b}
$$

where $m$ is the mass of the particle, $P$ is the pressure, $\rho$ is the density, $d / d t$ refers to the material derivative and $\nabla_{a} W$ is the gradient of kernel. Effective viscosity term in the equation is represented as;

$$
\begin{gathered}
\Pi_{a b}{ }^{e f f}=-\frac{K^{e f f} v_{s i g} \mathbf{v}_{a b} \cdot \mathbf{r}_{a b}}{\rho_{a b}\left|\mathbf{r}_{a b}\right|} \\
K^{e f f}=\left(\frac{1}{\rho} F\left(\left|\bar{D}_{a b}\right|\right)\left(\frac{112}{15 h v_{s i g}}\right)\right.
\end{gathered}
$$

$K^{e f f}$ coefficient depends upon the second invariant of the stress tensor defined in Equation 5.7. The dynamic viscosity is one of the coefficients described in artificial viscosity term in Equation 2.32; however, bi-viscosity model defined the dynamic viscosity in $F\left|\bar{D}_{a b}\right|$ term (presented in Equation 5.4). The shear rate tensor D (Eq. 5.1) is calculated by the first derivative of velocity in SPH context is shown below.

$$
(\nabla \cdot \mathbf{v})_{a}=\sum_{b} \frac{m_{b}}{\rho_{b}}\left(\mathbf{v}_{b}-\mathbf{v}_{a}\right) \cdot \nabla W_{a b}
$$

The signal velocity, $v_{\text {sig }}$, is defined by (Monaghan 2005)

$$
v_{s i g}=c_{a}+c_{b}-4 \frac{v_{a b} \cdot r_{a b}}{\left|r_{a b}\right|}
$$

where $\mathrm{c}$ is the speed of sound values for particle $a$ and $b$. 
The change in the fluid density is calculated by using the continuity equation in SPH context is shown below.

$$
\frac{d \rho_{a}}{d t}=\sum_{b} m_{b} v_{a b} \nabla_{a} W_{a b}
$$

Inter-particle spacing is the initial distance between the particles in SPH. This distance is fixed for boundary particles however it may change during the simulation for the fluid particles. Initially, particle spacing regulates the number of particles used for the simulation and studies showed that with increasing number of particles, the computational time increases significantly. For example, computational time required to simulate a $3 \mathrm{D}$ problem for a Newtonian fluid which is represented with more than 400,000 particles can take couple of days to complete (St-Germain, et al. 2012). Compared to FEM, computational speed of SPH is much slower; especially for higher number of particles. However, all of the simulations conducted have small volumes therefore the number of particles did not affect the computational speed significantly.

The sensitivity of the model to particle spacing and time step is presented in the following figures. It can be observed that the number of particles did not affect the final profile of the tailings significantly because the fluid has a higher viscosity therefore the velocity of the particles are smaller compared to Newtonian fluids.

Inter particle spacing for the numerical tests are determined based on the initial geometry. For example, the first tests the particle spacing in the $\mathrm{x}$ direction $(\mathrm{dx})$ is selected based on the diameter of pipe while for deposition tests using a funnel, $d x$ was set to $0.006 \mathrm{~m}$. Particle spacing in $\mathrm{z}$ direction $(\mathrm{dz})$ is decreased for the last single layer and for multi-layer deposition tests mainly because the volume used in these 
experimental tests are smaller. However, Figure 5.3 proven that with larger particle spacing, similar final geometry would be achieved.

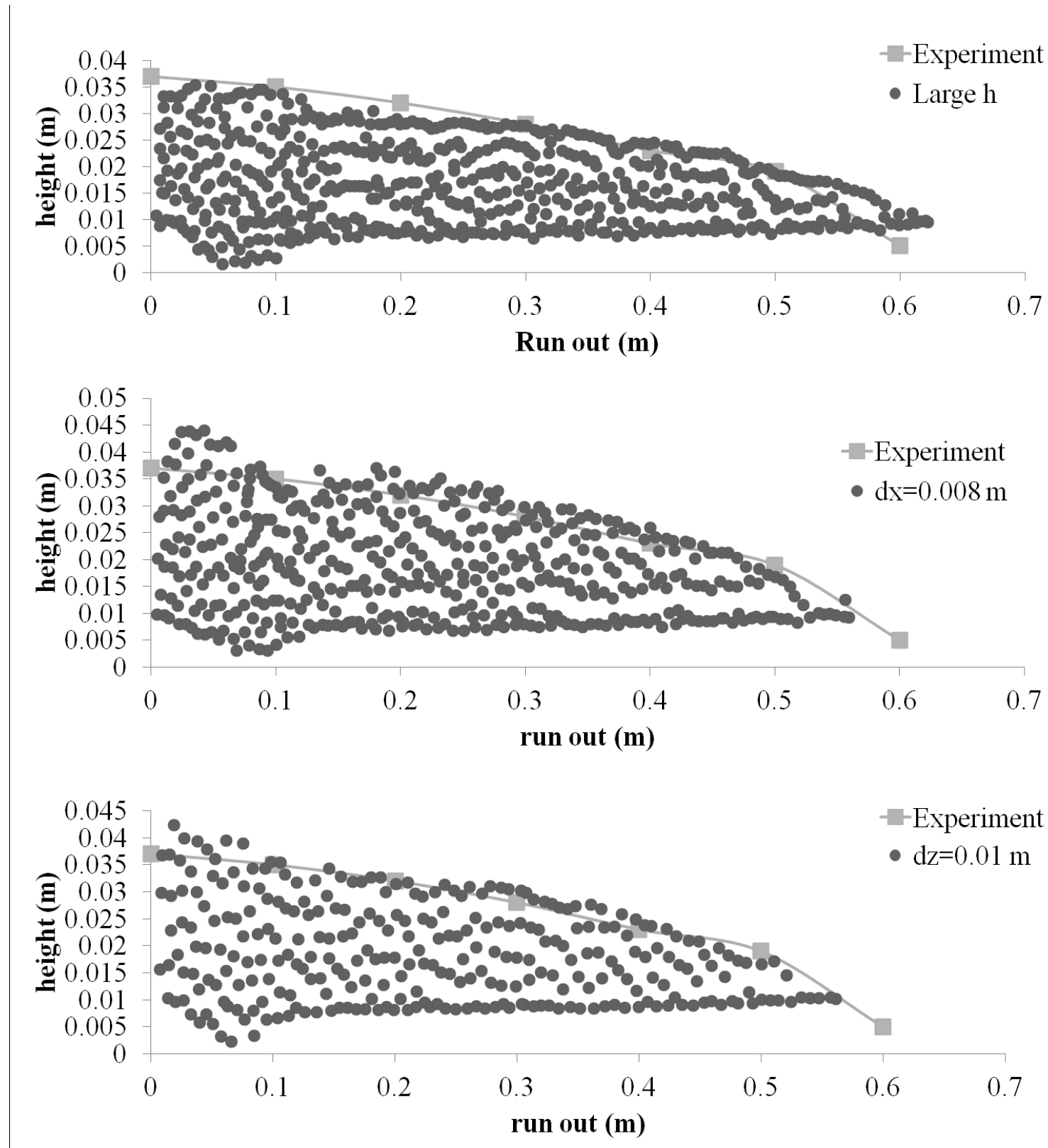

Figure 5.3: Final profile of tailings with various number of particles (476, 357 and 236 respectively) 


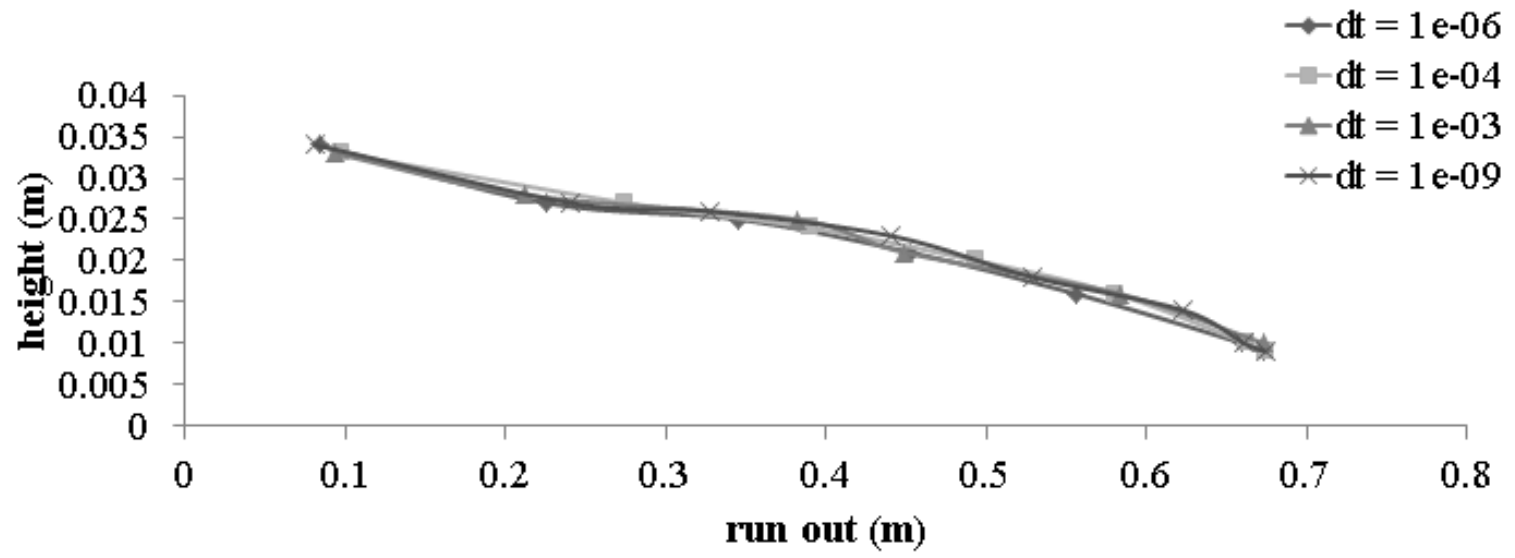

Figure 5.4: Sensitivity analysis of the proposed model to time stepping.

\subsubsection{Equation of State}

The general assumption in the fluid simulations is to assume the material is incompressible. However, for the simulation of fluids in $\mathrm{SPH}$, the particles are treated as weakly compressible fluids in order to avoid solving Poisson's equation for incompressible fluids at each time step. There are some conditions to be adjusted to make sure that the time step is reasonable; such as lowering the speed of sound so that the compressibility is adjusted. As explained in Chapter 2, the density variations in the code are calculated by using Continuity equation (Eq. 5.10). The density variations in the program should be less than $1 \%$, in order to provide this condition, the speed of sound selected must be about ten times faster than the maximum fluid velocity. The Equation of State describes the relationship between the pressure and the density (Gómez-Gesteira, et al. 2010).

$$
P=B\left[\left(\frac{\rho}{\rho_{0}}\right)^{7}-1\right]
$$

where

$$
B=c_{0}^{2} \rho_{0} / 7
$$


Where $\rho_{0}$ is the reference density and equal to $1000\left(\mathrm{~kg} / \mathrm{m}^{3}\right)$ for water and $c_{0}$ is the speed of sound at the reference density and has a unit of $\mathrm{m} / \mathrm{s}$.

Solving the Equation of State for weakly compressible fluids requires less time but it also demands unreasonable small time steps in order to solve the model. By imposing low compressibility which is accomplished by lowering the speed of sound, the disadvantage can be reduced.

The maximum speed in bulk flow is higher in Newtonian fluids compared to nonNewtonian fluids. For the Newtonian fluids, the speed of sound values was chosen as $42.5 \mathrm{~m} / \mathrm{s}$; however, the chosen value for non-Newtonian fluid is much smaller for specific model. In order to obtain the correct values, 'fill the cup' tests were performed where the particles are initially positioned in a full cup and their hydrostatic pressures were obtained from the program. The values are then compared with the theory.

$$
P=\rho g(h-z)
$$

where $\mathrm{g}$ is the gravitational acceleration, $\rho$ is the density of the particles and $\mathrm{z}$ is the height of the particle. The initial positions of the particles in a full cup are represented below. 


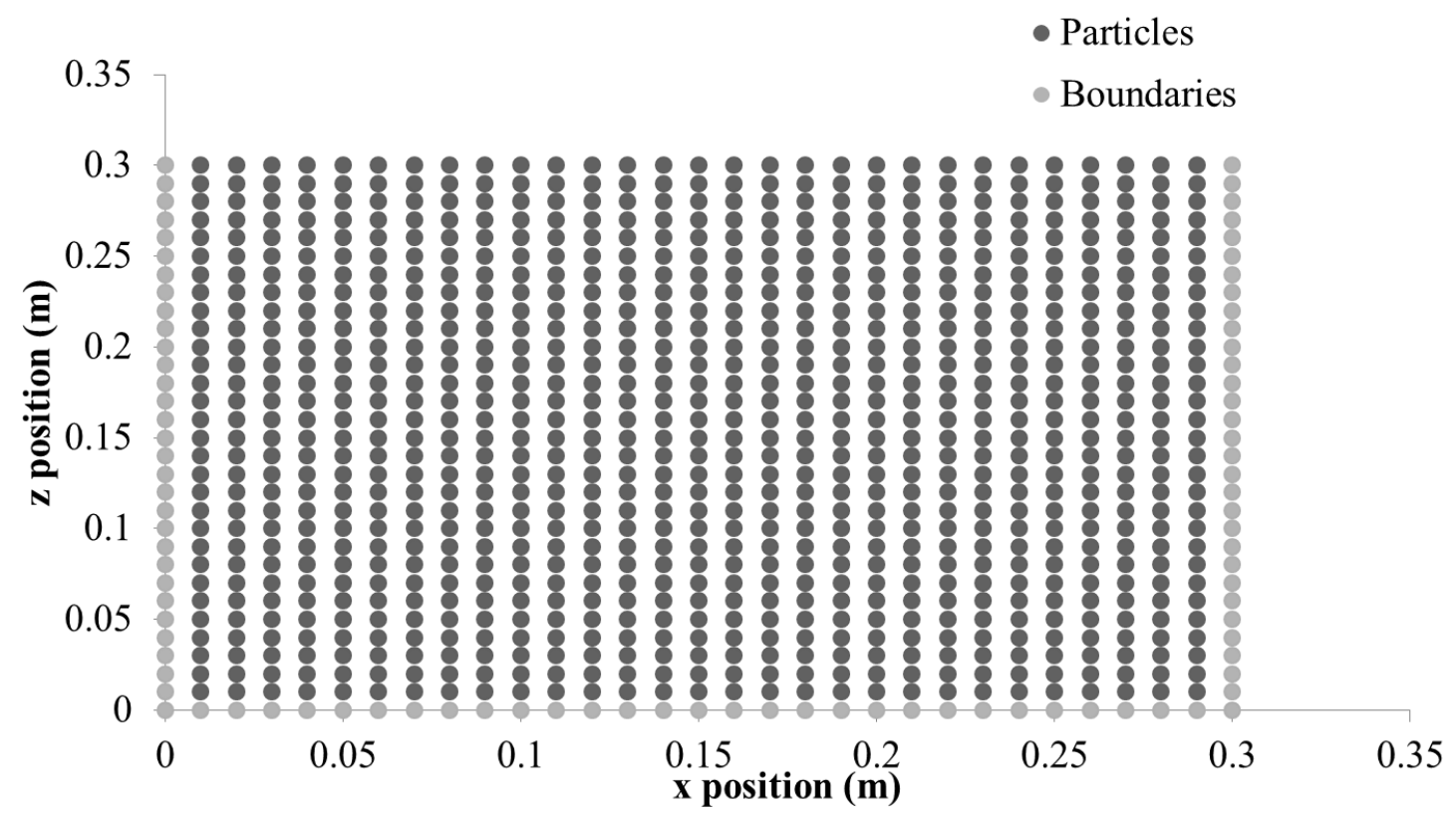

Figure 5.5: Initial particle positions for 'Fill the cup' tests

The height and the width of the cup is $30 \mathrm{~cm}$ and the distance between particles are determined to be $1 \mathrm{~cm}$ to be precise in both directions. Total of 841 particles are used to replace the fluid and the results of hydrostatic pressure values are revealed below.

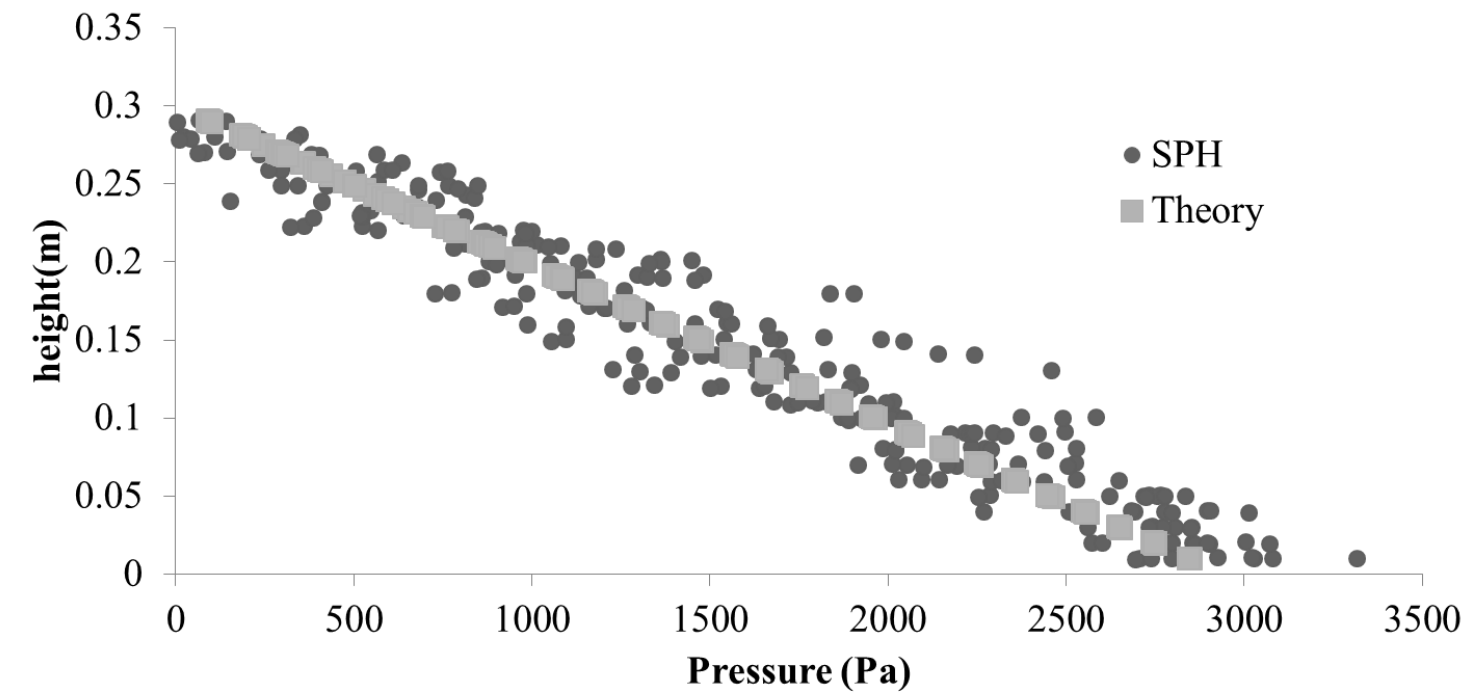

Figure 5.6: Pressures vs. Particle heights for Newtonian fluid. 
The dynamic viscosity values of high density tailings used in the experiments ranging from 0.1 to 1.0 Pa.s depending on their yield stress values (data obtained from Henriquez and Simms (2009)). Higher the yield stress values, higher the resistance of the fluid will be. In order to determine the correct $c_{0}$ value hydrostatic pressure are calculated by using different speed of sound values for specific viscosities.
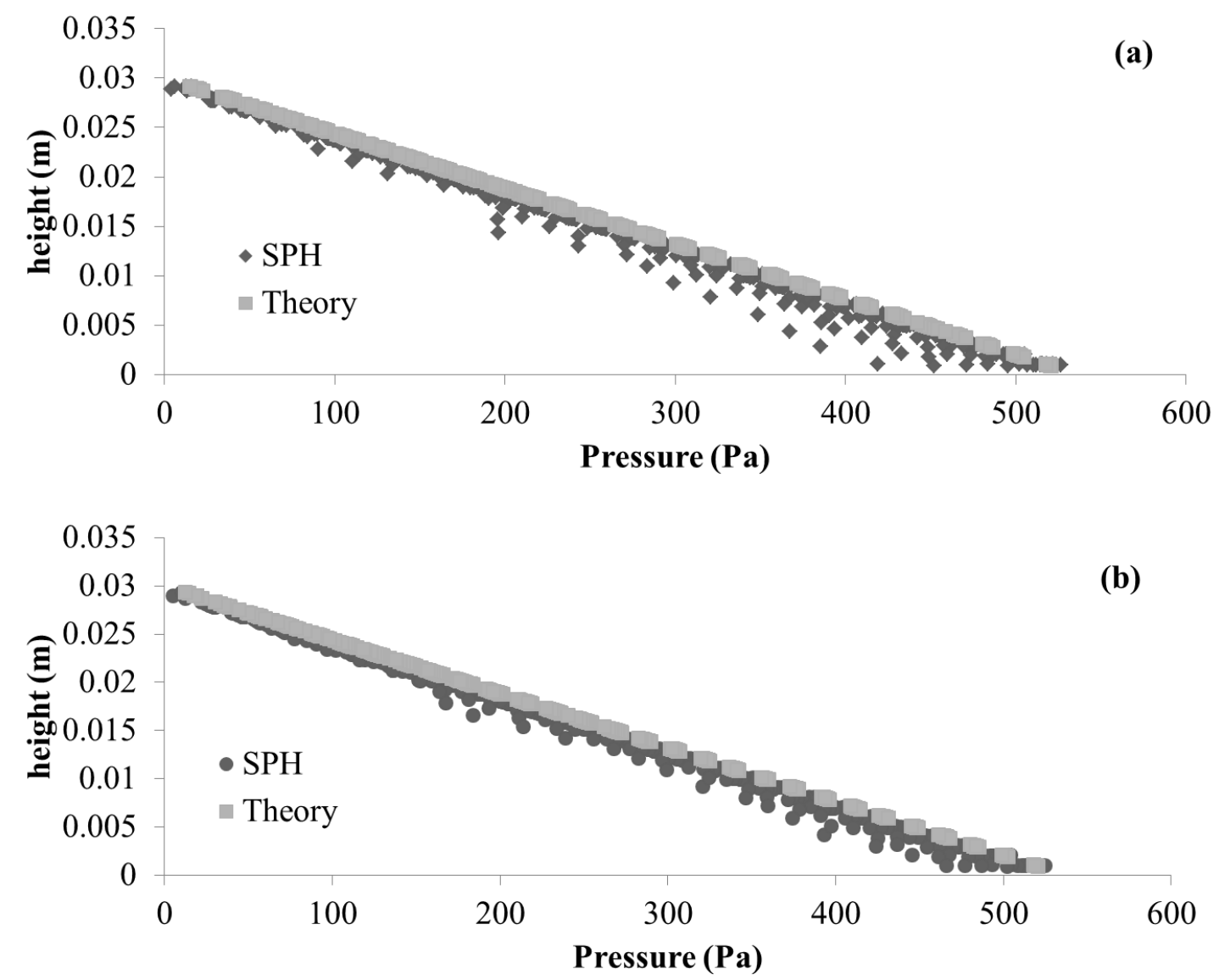

Figure 5.7: Pressure vs. Particle height for Non-Newtonian fluids $\left(\rho=1823.8 \mathrm{~kg} / \mathrm{m} 3 \& \tau_{\mathrm{y}}=45 \mathrm{~Pa}\right.$ ) (a) $c_{0}$ is $10 \mathrm{~m} / \mathrm{s}$ and the viscosity is 0.5 Pa.s (b) $c_{0}$ is $14 \mathrm{~m} / \mathrm{s}$ and the viscosity is 0.8 Pa.s 


\subsubsection{Density Re-Initialization}

The dynamics of SPH generally produce realistic results however the pressure field of the particles may create large pressure oscillations during the simulations. In order to prevent this difficulty, several approaches have been considered such as correcting the kernel or developing an incompressible solver. Applying a filter over the density of the particles is one of the most straight forward and computationally least expensive methods. This filter corrects the density values of the particles and re-assigns a density for each particle (Gómez-Gesteira, et al. 2010).

In SPH method, each particle has a constant mass, $m_{b}$, and mass conservation is satisfied if the number of particles are constant (Eq. 5.10). The consistency between mass, density and the occupied area cannot be enforced; to avoid the problem density field should be periodically reinitialized by using the equation below. First order correction scheme, Moving Least Square (MLS), method applied by Colagrossi and Landrini (2003) to reproduce the density field during the simulations.

$$
\rho_{a}^{\text {new }}=\sum_{b} \rho_{b} W_{a b}{ }^{M L S} \frac{m_{b}}{\rho_{b}}=\sum_{b} m_{b} W_{a b}
$$

The selected kernel is very important for the procedure because if $\sum_{b} W_{a b} \neq 1$ at a point $\mathrm{x}_{\mathrm{a}}$ then the use of the density filter would present some additional problems especially when the particles is approaching to a boundary. The number of particle neighbours seen by the boundary particles diminishes as a result the computed densities are lower than the original input. Therefore, the program would estimate the wrong pressure values, corrupting the entire field progressively (Colagrossi and Landrini 2003). 
The corrected kernel is in two dimensions evaluated as follows;

$$
W_{a b}^{M L S}=W_{b}^{M L S}\left(r_{a}\right)=\beta\left(r_{a}\right)\left(r_{a}-r_{b}\right) W_{a b}
$$

where $r_{a, b}$ are the positions vectors for particles $a \& b$, and $\beta$ is the correction vector. The correction vector is given by;

$$
\beta\left(r_{a}\right)=\left[\begin{array}{l}
\beta_{0} \\
\beta_{1 x} \\
\beta_{1 z}
\end{array}\right]=A^{-1}\left[\begin{array}{l}
1 \\
0 \\
0
\end{array}\right]
$$

where

$$
A\left(x_{a}\right)=\sum_{b} W_{b}\left(x_{a}\right) A_{a b}
$$

And the matrix A is defined as;

$$
A_{a b}=\left[\begin{array}{ccc}
1 & \left(x_{a}-x_{b}\right) & \left(z_{a}-z_{b}\right) \\
\left(x_{a}-x_{b}\right) & \left(x_{a}-x_{b}\right)^{2} & \left(x_{a}-x_{b}\right)\left(z_{a}-z_{b}\right) \\
\left(z_{a}-z_{b}\right) & \left(z_{a}-z_{b}\right)\left(x_{a}-x_{b}\right) & \left(z_{a}-z_{b}\right)^{2}
\end{array}\right]
$$

where $\mathrm{x} \& \mathrm{z}$ is the particle positions. This procedure is applied at each 30 time step to reconstruct the consistency between the density, mass and occupied area. It increases the computing time slightly but it is necessary to obtain satisfactory results. Crespo (2008) applied this re-initialization method to a simple 2D dam break problem. The impact of the filter on the water front against the wall can be seen in Figure 5.8 below. 


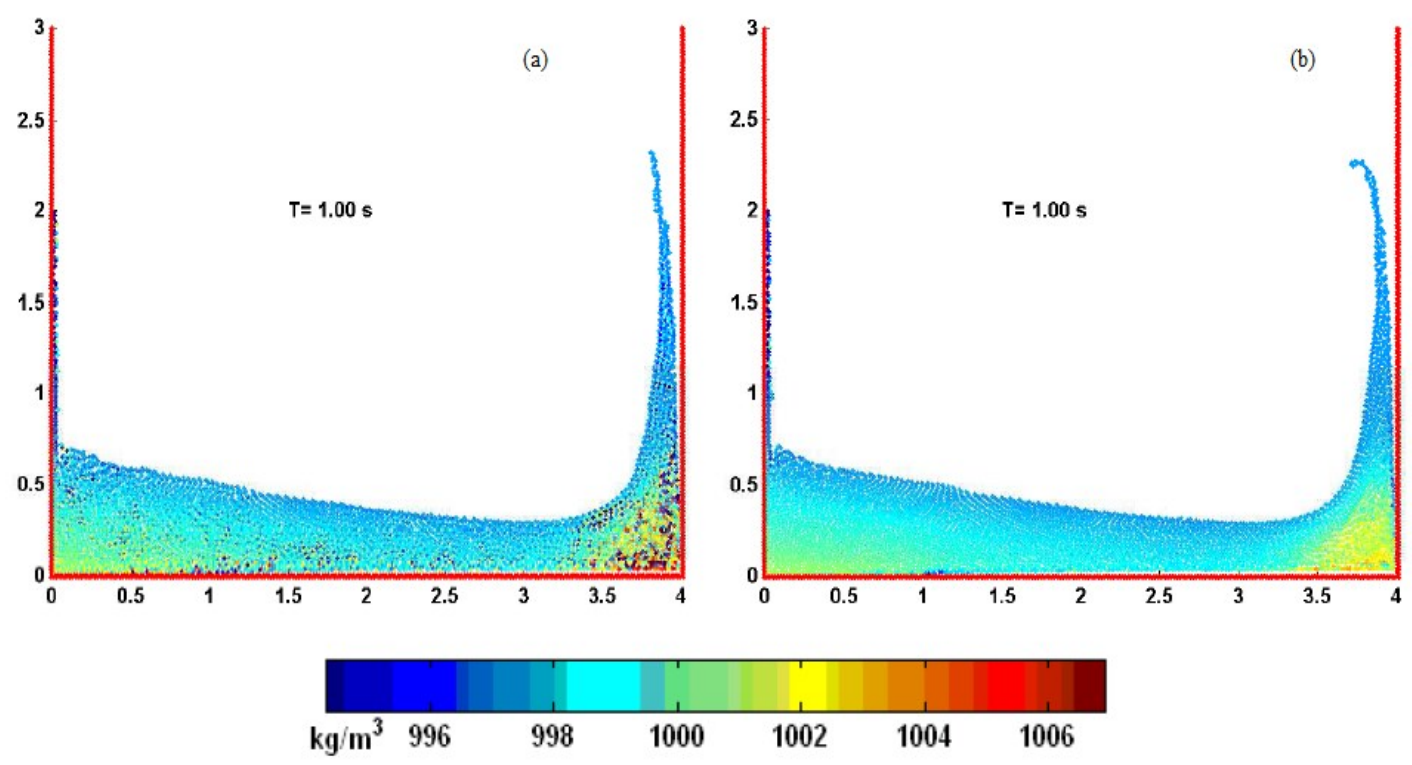

Figure 5.8: Density field for 2-D dam break showing effect of density (a) without density filter (b) with First order-MLS filter (Crespo 2008).

\subsubsection{Time Stepping}

There are various time stepping algorithms are available in SPHYSICS: (i) PredictorCorrector scheme; (ii) the Verlet algorithm; (iii) the Symplectic algorithm and (iv) Beeman scheme. The following equations represent the momentum, density, position and the energy conservations in the following form (Gómez-Gesteira, et al. 2010).

$$
\begin{aligned}
& \frac{d v_{a}}{d t}=F_{a} \\
& \frac{d \rho_{a}}{d t}=D_{a} \\
& \frac{d r_{a}}{d t}=V_{a} \\
& \frac{d e_{a}}{d t}=E_{a}
\end{aligned}
$$


Where $V_{a}$ represents the velocity contribution from particle a and the neighboring particles. The Predictor-Corrector scheme is one of the most common approaches and it is also selected for this research.

This scheme predicts the evolution in time as (Crespo 2008; Gómez-Gesteira, et al. 2010)

$$
\begin{aligned}
& v_{a}^{n+1 / 2}=v_{a}{ }^{n}+\frac{\Delta t}{2} F_{a}{ }^{n} \\
& \rho_{a}^{n+1 / 2}=\rho_{a}{ }^{n}+\frac{\Delta t}{2} D_{a}{ }^{n} \\
& r_{a}^{n+1 / 2}=r_{a}^{n}+\frac{\Delta t}{2} V_{a}^{n} \\
& e_{a}^{n+1 / 2}=e_{a}^{n}+\frac{\Delta t}{2} E_{a}{ }^{n}
\end{aligned}
$$

Pressure equation $P_{a}^{n+1 / 2}=f\left(\rho_{a}{ }^{n+1 / 2}\right)$ is calculated according to Eq. 5.11. These values are then corrected using the forces at the half time step as follows;

$$
\begin{aligned}
& {v_{a}{ }^{n+1 / 2}}^{=} v_{a}{ }^{n}+\frac{\Delta t}{2} F_{a}^{n+1 / 2} \\
& \rho_{a}^{n+1 / 2}=\rho_{a}^{n}+\frac{\Delta t}{2} D_{a}^{n+1 / 2} \\
& r_{a}^{n+1 / 2}=r_{a}^{n}+\frac{\Delta t}{2} V_{a}^{n+1 / 2} \\
& e_{a}^{n+1 / 2}=e_{a}^{n}+\frac{\Delta t}{2} E_{a}^{n+1 / 2}
\end{aligned}
$$

The values are finally calculated at the end of time step following; 


$$
\begin{aligned}
& v_{a}^{n+1}=2 v_{a}{ }^{n+1 / 2}-v_{a}{ }^{n} \\
& \rho_{a}^{n+1}=2 \rho_{a}^{n+1 / 2}-\rho_{a}{ }^{n} \\
& r_{a}^{n+1}=2 r_{a}^{n+1 / 2}-r_{a}^{n} \\
& e_{a}^{n+1}=2 e_{a}^{n+1 / 2}-e_{a}{ }^{n}
\end{aligned}
$$

Finally, the pressures are calculated using $P_{a}^{n+1}=f\left(\rho_{a}^{n+1}\right)$

The variable time step in SPH is dependent on several conditions: Courant-FredrichLevy condition, the forcing terms and the viscous diffusion terms. The time step is calculated using the formulation below (Gómez-Gesteira, et al. 2010).

$$
\begin{gathered}
\Delta t=0.3 \min \left(\Delta t_{\mathrm{f}}, \Delta \mathrm{t}_{\mathrm{cv}}\right) \\
\Delta t_{f}=\min _{a} \sqrt{h /\left|f_{a}\right|} \\
\Delta t_{c v}=\min _{a} \frac{h}{c_{s}+\max _{b}\left|\frac{h \mathrm{v}_{a b} \mathrm{r}_{a b} \mid}{\mathrm{r}_{a b}{ }^{2}}\right|}
\end{gathered}
$$

where $\left|f_{a}\right|$ is the force per unit mass.

In $\mathrm{SPH}$, the computational domain is divided into square cells as shown in Figure 5.9. The dimensions of these cells are determined by the smoothing length $h, 2 h$ to be precise. The particles inside a cell only interact with the particles in neighbouring cells; therefore the number of calculations and the computational time decrease significantly. 


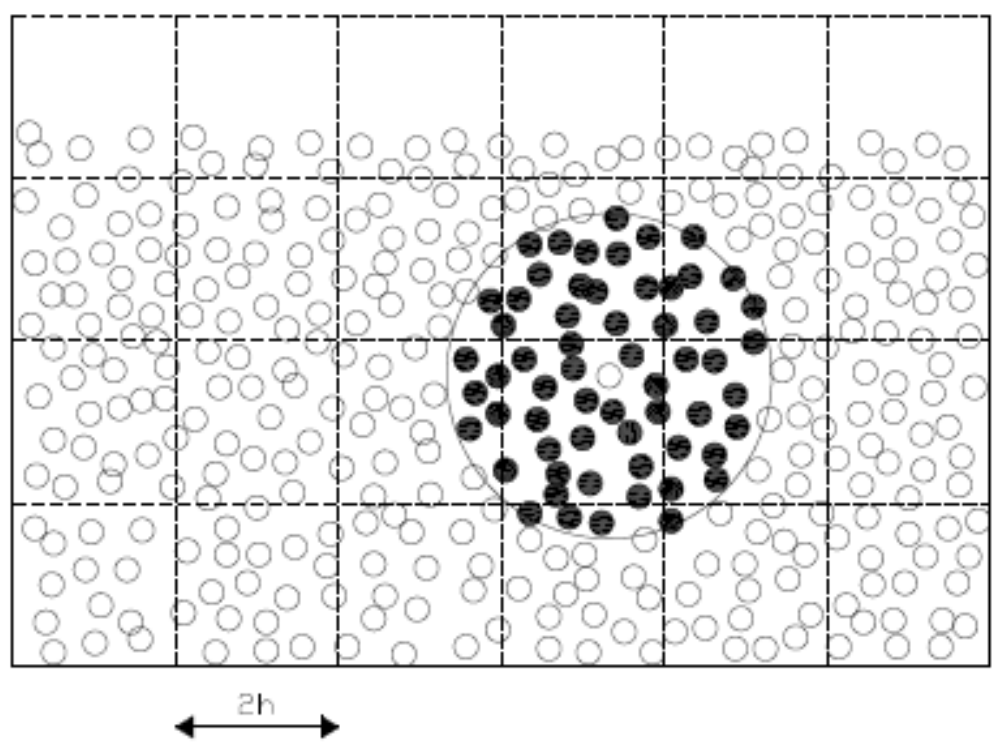

Figure 5.9: Domain divided into $2 \mathrm{~h}$ cells and the particle only interacts with the neighbouring particle marked by black dots (Gómez-Gesteira, et al. 2010).

In two dimensional simulations, the code sweeps though the cells in $\mathrm{x}$ direction for each level in $\mathrm{z}$ direction. In order to minimise the repeating particle interactions, for each cell the E, N, NW and NE neighbouring cells are checked (Fig. 5.10). The interaction with other directions (W, S, SW and SE) in each cell already calculated using reverse interactions. For example, the interaction between the cell $i k(5,3)$ and the cell below $i k$ $(5,2)$ in the figure (Figure 5.8) already calculated when cell below $i k(5,2)$ is considered as a main cell. 


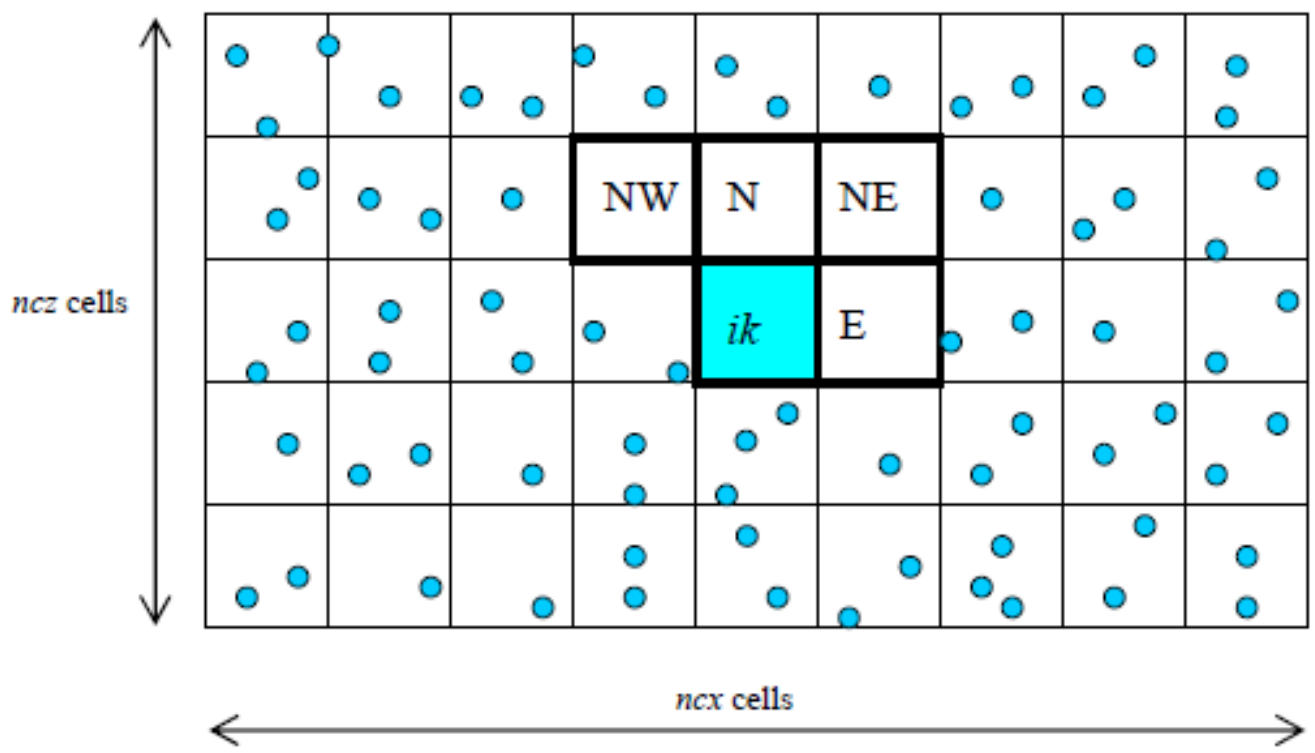

Figure 5.10: The code sweeping through each cell starting from left corner (Gómez-Gesteira, et al. 2010).

In conclusion, there are two component of solution considered in SPHYSICS: the first one tracks the boundary particles which is upgraded partially at each time step, especially if moving objects are available in the model such as floating objects and gates, and the second one is correspond to fluid particles which is upgraded completely at each time step (Crespo 2008; Gómez-Gesteira, et al. 2010).

\subsection{Test Cases}

The model was tested to determine the capability of proposed algorithm for the solution of non-Newtonian fluid problems. The selected test cases includes both the experimental work analysed in Chapter 4 and the experimental work conducted by Henriquez and Simms (2009) for single and multi-layer deposition tests. 


\begin{tabular}{|l|c|l|}
\hline & Test & Description \\
\hline Single Layer & 1 & Flume test \#14 using Bulyanhulu tailings on a horizontal bed \\
\cline { 2 - 3 } & 2 & Large volume deposition test using a gate \\
\cline { 2 - 3 } & 3 & Large volume deposition test using a funnel \\
\cline { 2 - 3 } & 4 & Transient flow prediction on a single layer \\
\cline { 2 - 3 } & 5 & Flume test on a sloped plane $(0.57$ and 1.14 degrees) \\
\hline Multi-Layer & 6 & Flume test using various parameters on a horizontal plane \\
\hline
\end{tabular}

Table 5.1: The summary of numerical experiments conducted using SPH.

Experimental work presented in Chapter 4, mostly focuses on the depositional behaviour of high density tailings with various flow rates. Even though, deposition time and total volume varied, all the material was poured on top of a horizontal bed through a small pipe opening. Henriquez and Simms (2009) studied the change in the equilibrium profiles for different deposition methods such as using a funnel (larger opening) and a gate; including transient flow prediction. The experimental data required multi-layer simulations and depositional behaviour on a sloped bed was obtained from Henriquez and Simms (2009); since the experiments were not conducted in laboratory.

\subsubsection{Single Layer Flume Tests}

\section{a. Large volume deposition on a horizontal bed}

The first test analyzed is the single layer deposition of tailings in a flume with a flat bed. The numerical results are compared with the experiment conducted (test \#14, explained thoroughly in section 4.4.1) using Bulyanhulu high density tailings since the experimental results give the best agreement with the Lubrication Theory.

Total volume of the tailings are $9.0 \mathrm{~L}$ where the density of the tailings is $1824 \mathrm{~kg} / \mathrm{m}^{3}$ and the yield stress values acquired from slump test is determined as $45 \mathrm{~Pa}$. The pipe is located at 0.2 metres above the bed and the particles are initially positioned at $0.5 \mathrm{~m}$ 
height. The diameter of the pipe is $7.3 \mathrm{~mm}$ hence in order to control the deposition total volume is divided into five sections. In each section the height of the particles in the pipe is 1.5 metres and they are completely flown out of the pipe in 3.5 seconds. The particle positions, densities and pressure values are recorded and for the next simulation they are read into the program from a file. The initial position of the particles for each section is shown in Figure 5.11.

The key SPH parameters chosen for the specific test case is displayed in the following table.

\begin{tabular}{|l|l|l|}
\hline Geometry of the flume & Fluid & Time \\
\hline$d x=0.01 \mathrm{~m}$ & $d x=0.0073 \mathrm{~m}$ & $d t=1 \mathrm{E}-06 \mathrm{~s}$. \\
$d z=0.01 \mathrm{~m}$ & $d z=0.02 \mathrm{~m}$ & run time $=16.5 \mathrm{~s}$. \\
$\mathrm{d}_{\text {pipe }}=7.3 \mathrm{~mm}$ & $\tau_{\mathrm{y}}=45 \mathrm{~Pa}$. & \\
smoothing length $(h)=8.89 \mathrm{~mm}$ & $\alpha=40$ & \\
B.C.: Dynamic & Viscosity $(\mu)=0.8$ Pa.s. & \\
& B=2700 & \\
\hline
\end{tabular}

Table 5.2: The key parameters chosen for single layer deposition of Bulyanhulu high density tailings in a flume (test \#1).

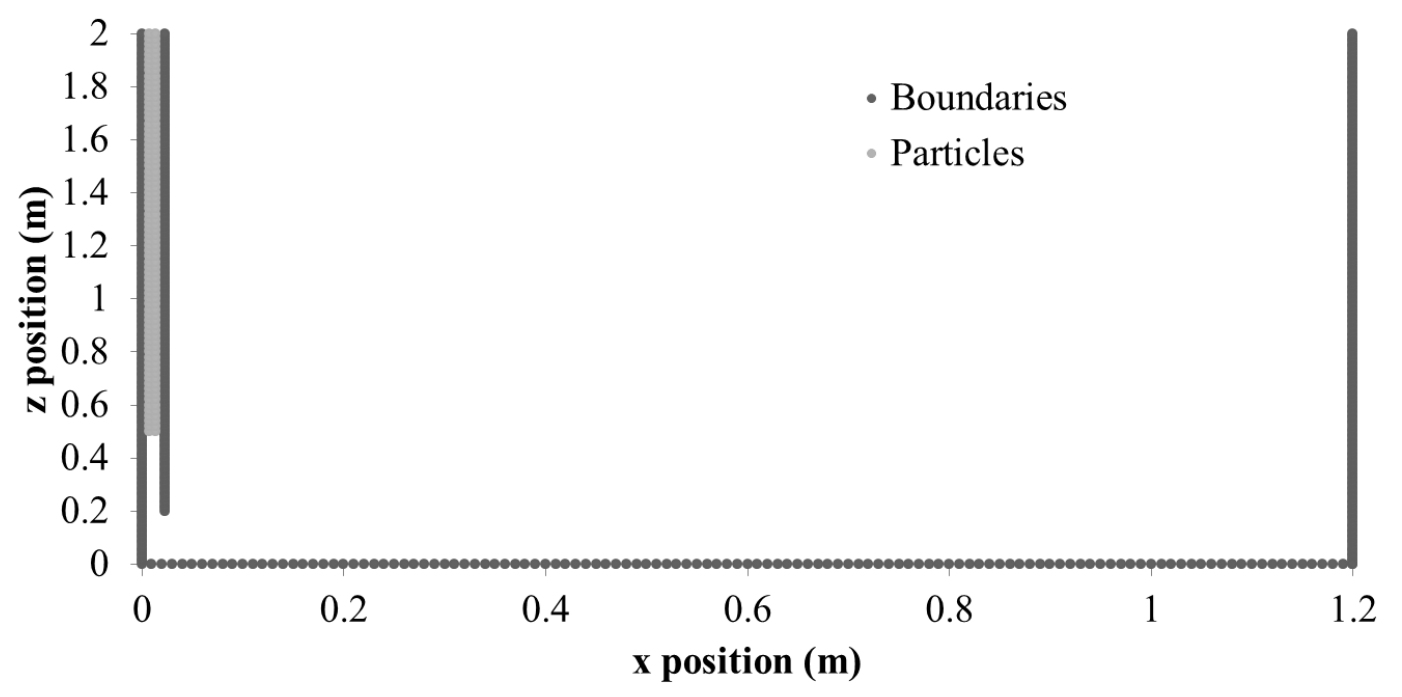

Figure 5.11: The initial particle and boundary positions for test \#1. 
As mentioned earlier, the volume was divided into five sections in order to control the deposition. In all of sections, the initial height of the particles is 1.5 metres and the diameter of the pipe is $7.3 \mathrm{~mm}$ where the particles are initially positioned. The final shape of the tailings at the end of the simulation is demonstrated in Fig. 5.12. The final shape is them compared with both Lubrication Theory (Eq. 3.3) and the experimental data acquired in the laboratory.

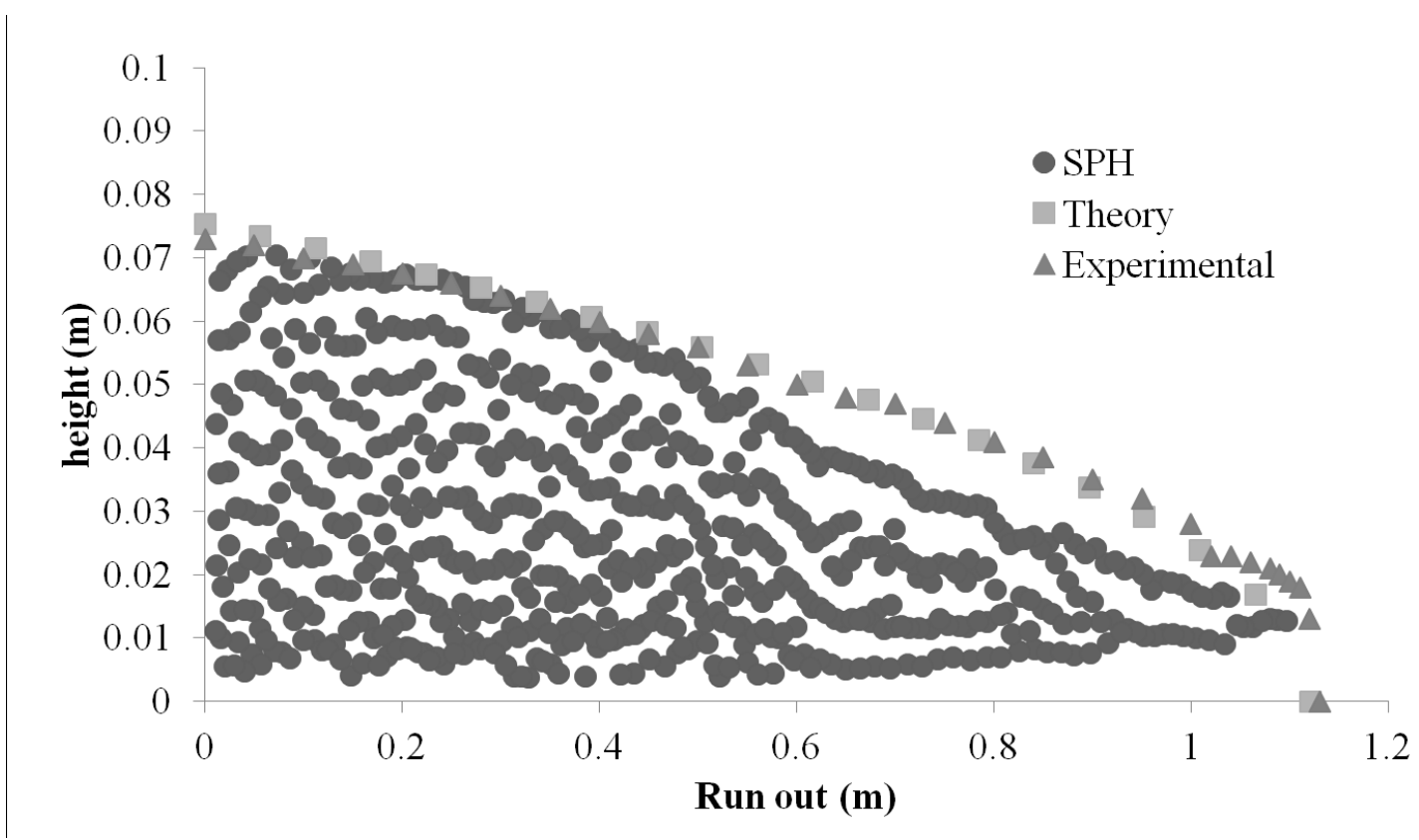

Figure 5.12: The final shape of the tailings at the end of the deposition.

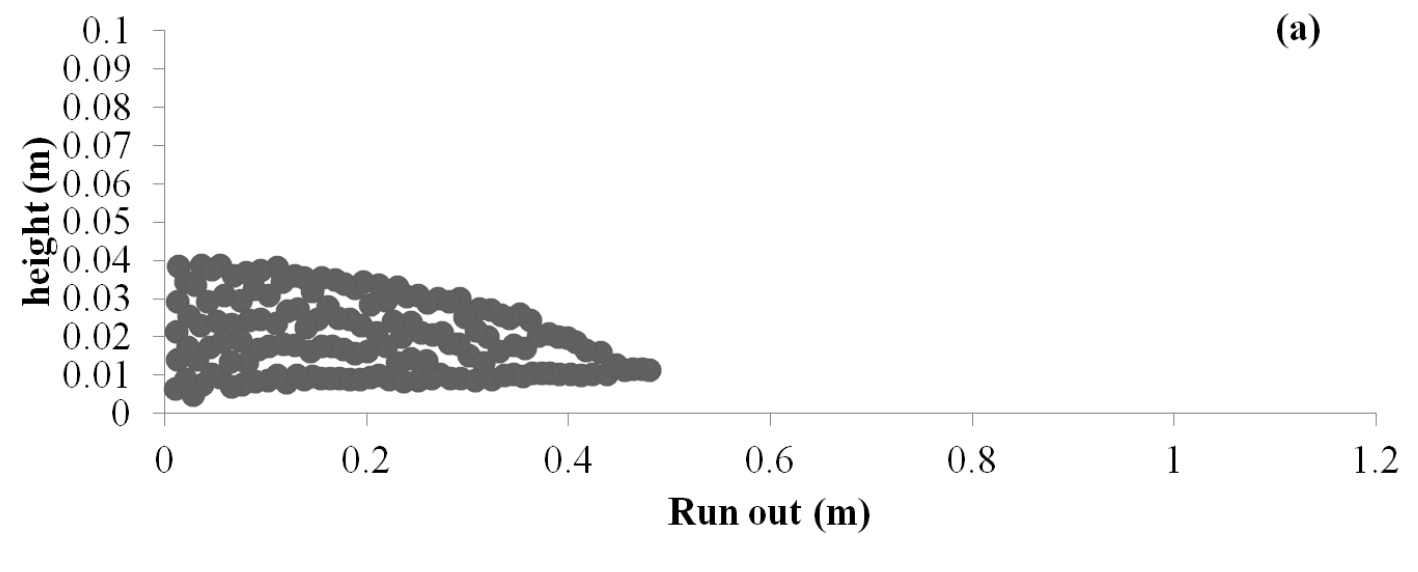



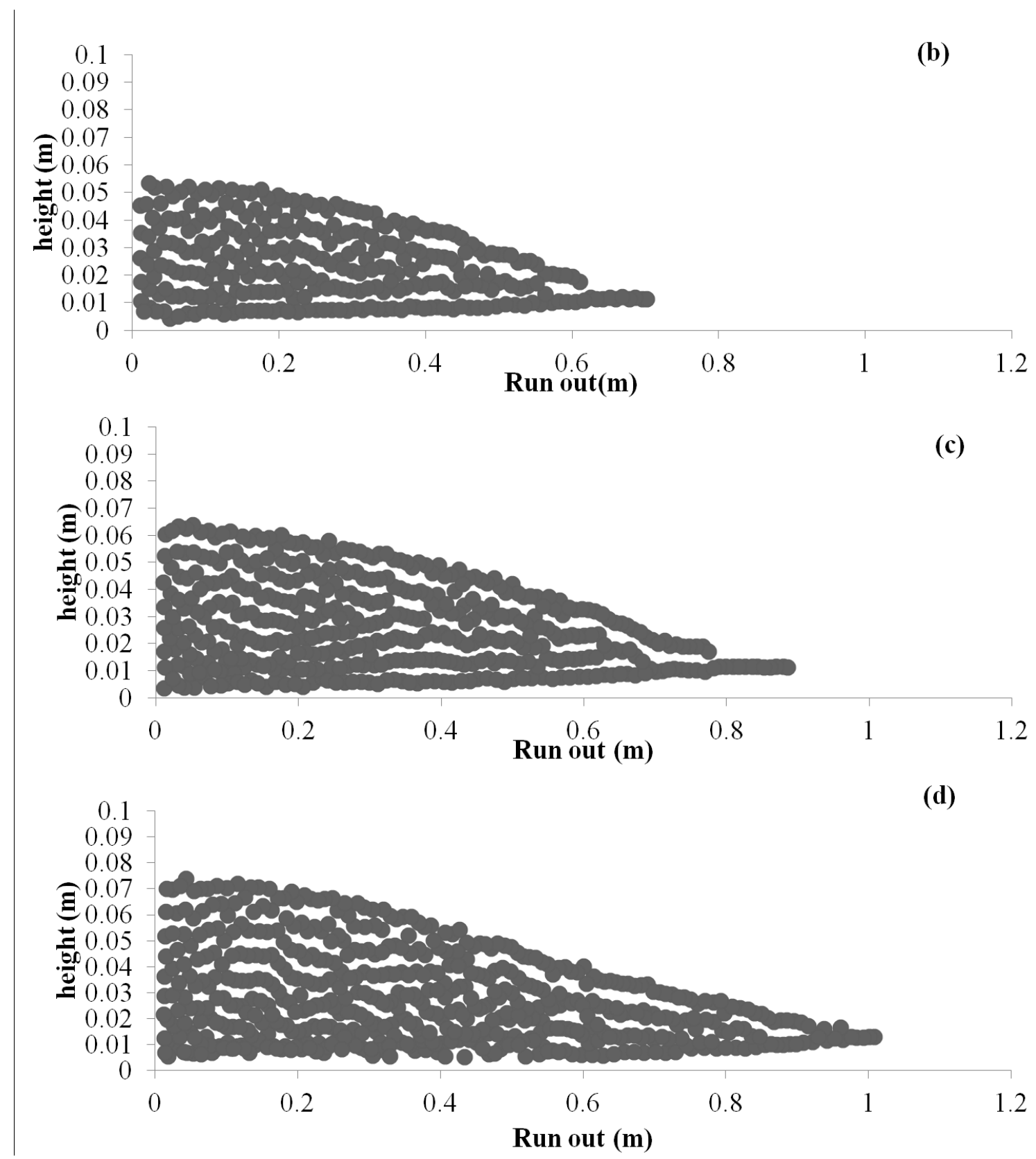

Figure 5.13: The shape of free surface at various times (a) $t=3.5 s$. (b) $t=7.0 s$ (c) $t=10.5 s$ (d) $t=14 s$.

The run out distance and the height of the stack is in good agreement with Lubrication Theory as well as the experimental data. The Lubrication Theory suggests that in the un-yielded region, no deformation will take place; as a result the particles come to a complete stop. In the proposed model, that specific region is assigned to a 
higher viscosity; decreasing the velocity of the particles. The flow rate of the particles when they exited the pipe is considerably high; therefore computational time required for a complete stop will be longer. Even though the particles are assigned to a higher viscosity, they will still continue to flow with slower velocities. In order to control the un-yielded region, the yield stress values for each particle are calculated and recorded at each time step. The results give the best agreement with the experimental data when more than $70 \%$ of the particles fall into the un-yielded region where the rate of run out is decreased to less than $0.01 \mathrm{~m} / \mathrm{s}$.

\section{b. Large volume deposition using a gate and funnel on a horizontal bed}

Henriquez and Simms (2009) evaluated the effect of the scale of the deposit on the overall slope angle of the tailings stack. They have found that the small scale depositions, generates steeper overall slope angles compared to the large volume depositions. Also, the effect of the deposition method (using a funnel and a gate) is also studied. The results demonstrated that final profiles are similar for small scale depositions but there is a significant difference on the final shape for large scale deposition. The same experiment adapted for larger volume deposition using a gate and a funnel; the initial geometry and the particle positions are demonstrated in Figures 5.14 and 5.16.

The experiments are conducted in a flume on a horizontal bed where the viscosity and the yield stress of the fluid are $0.65 P a . s$ and $36 P a$ respectively (the total volume of the tailings are approximately $5500 \mathrm{~cm}^{3}$ ). They key SPH parameters for both numerical tests are summarized in the flowing table. 


\begin{tabular}{|c|c|l|l|}
\hline & Geometry of the flume & Fluid & Time \\
\hline Gate & $d x=0.01 \mathrm{~m}$ & $d x=0.01 \mathrm{~m}$ & $d t=1 \mathrm{E}-06 \mathrm{~s}$. \\
& $d z=0.01 \mathrm{~m}$ & $d z=0.01 \mathrm{~m}$ & run time=3.0 s. \\
& $h=7.07 \mathrm{~mm}$ & $\mathrm{~B}=5000$ & \\
& D.B.C & $\alpha=40$ & \\
& & $\mu=0.65 \mathrm{~Pa} . \mathrm{s}$ & \\
\hline Funnel & $d x=0.01 \mathrm{~m}$ & $d x=0.006 \mathrm{~m}$ & $d t=1 \mathrm{E}-06 \mathrm{~s}$. \\
& $d z=0.01 \mathrm{~m}$ & $d z=0.01 \mathrm{~m}$ & run time $=3.0 \mathrm{~s}$. \\
& $\mathrm{d}_{\text {funnel }}=2.4 \mathrm{~cm}$ & $\mathrm{~B}=5000$ & \\
& $h=7.07 \mathrm{~mm}$ & $\alpha=40$ & \\
& D.B.C. & $\mu=0.65 \mathrm{~Pa} . \mathrm{s}$ & \\
& & & \\
\hline
\end{tabular}

Table 5.3: The key SPH parameter chosen for large volume tests using a funnel and a gate with yield stress and viscosity values $36 \mathrm{~Pa}$ and $0.65 \mathrm{~Pa}$.s respectively.

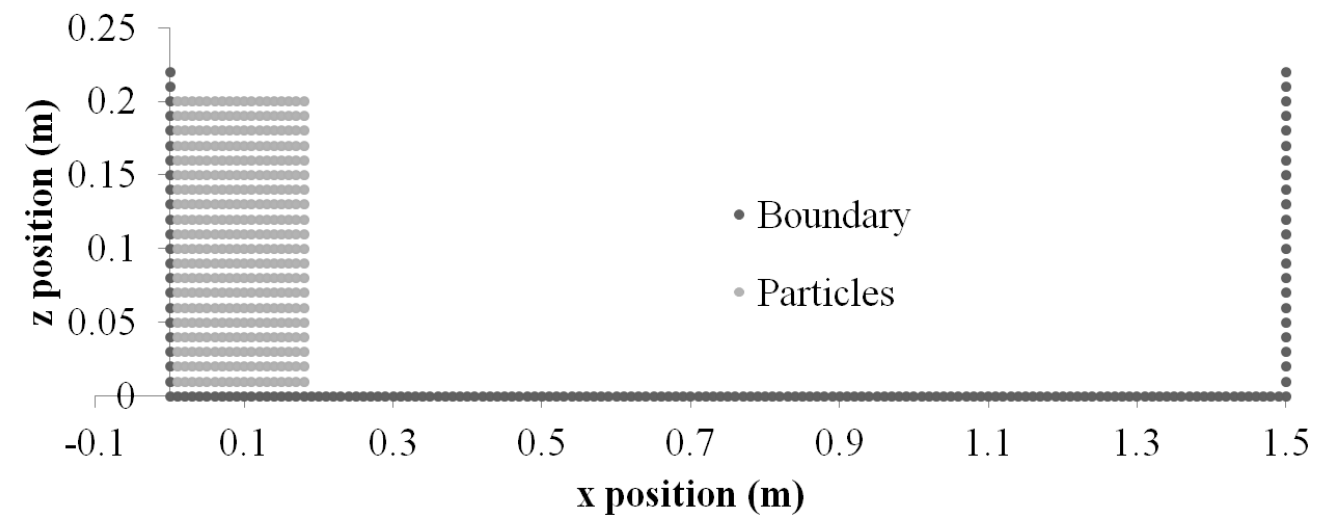

Figure 5.14: The initial position of the particles for the experiment $\# 2$ where the initial height of the particles is $0.2 \mathrm{~m}$ and the length is $0.18 \mathrm{~m}$.

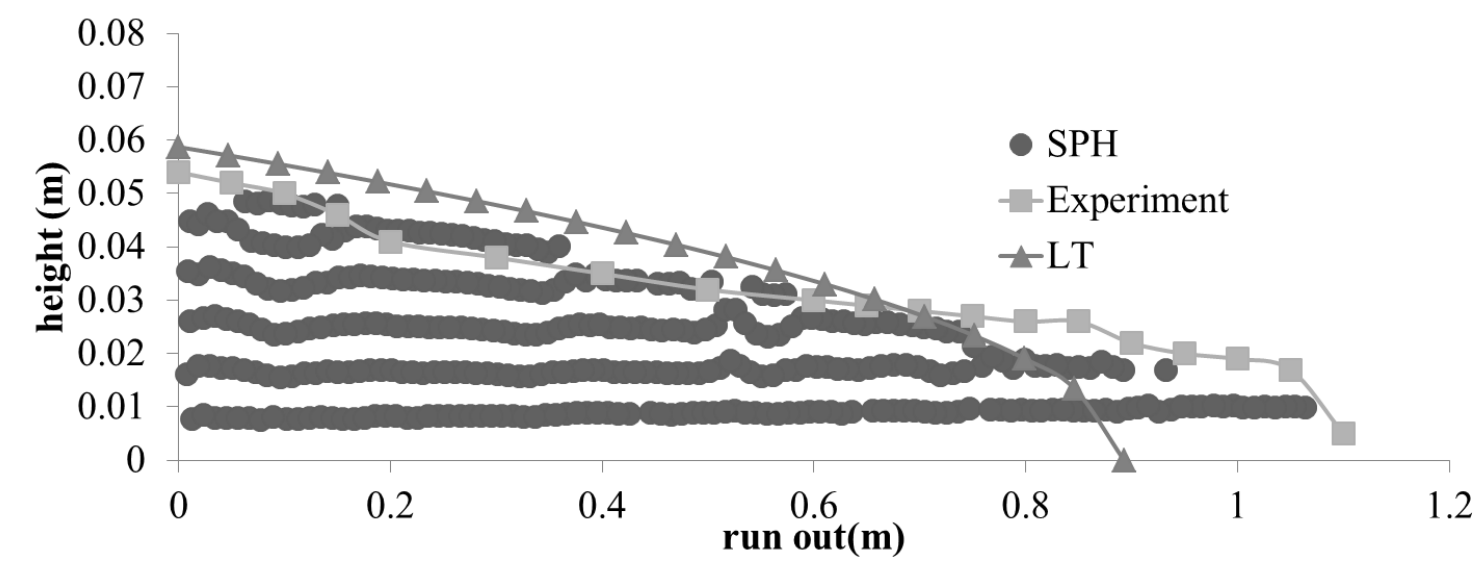

Figure 5.15: The final geometry of the experiment $\# 2$. 
The results indicate that the SPH gives a better agreement with the experimental data compared to Lubrication Theory (Figure 5.15). Similar to the first test, the volume of the fluid is divided into two sections to control the deposition and make sure that all of the particles are inside the boundaries. The funnel opening in the experiments is $2.4 \mathrm{~cm}$ long and in order to minimise the effect of inertia, the particles are positioned from the bottom of the flume instead of positioned inside the walls. Total run time is 3 seconds, and the final geometry of the fluid is displayed in Figure 5.17. Both of the tests showed good correlation with the experimental data.

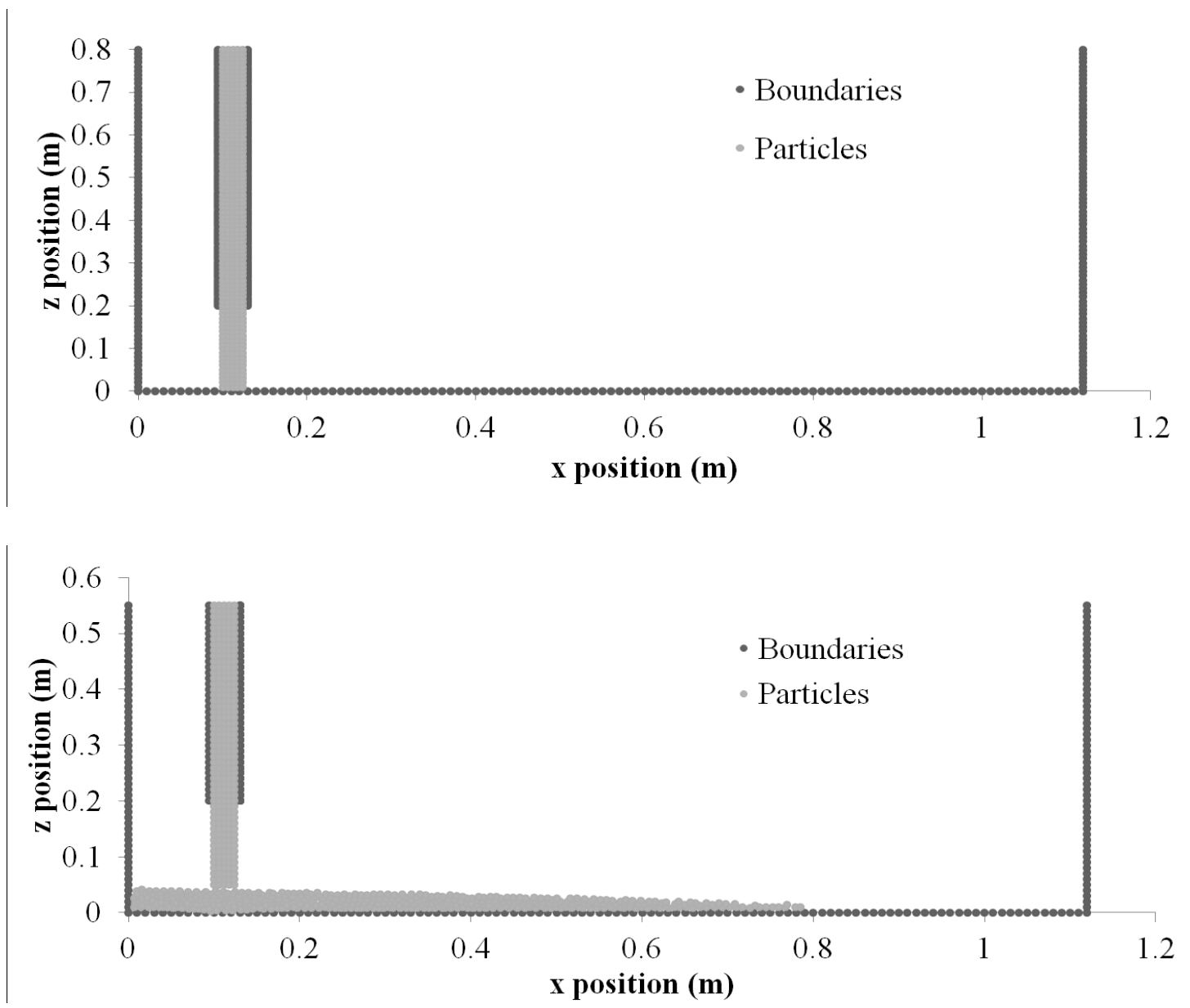

Figure 5.16: The geometry and the initial particle positions for test $\# 3$ (a) $t=0 \mathrm{~s}$ (b) $t=2.0 \mathrm{~s}$. 


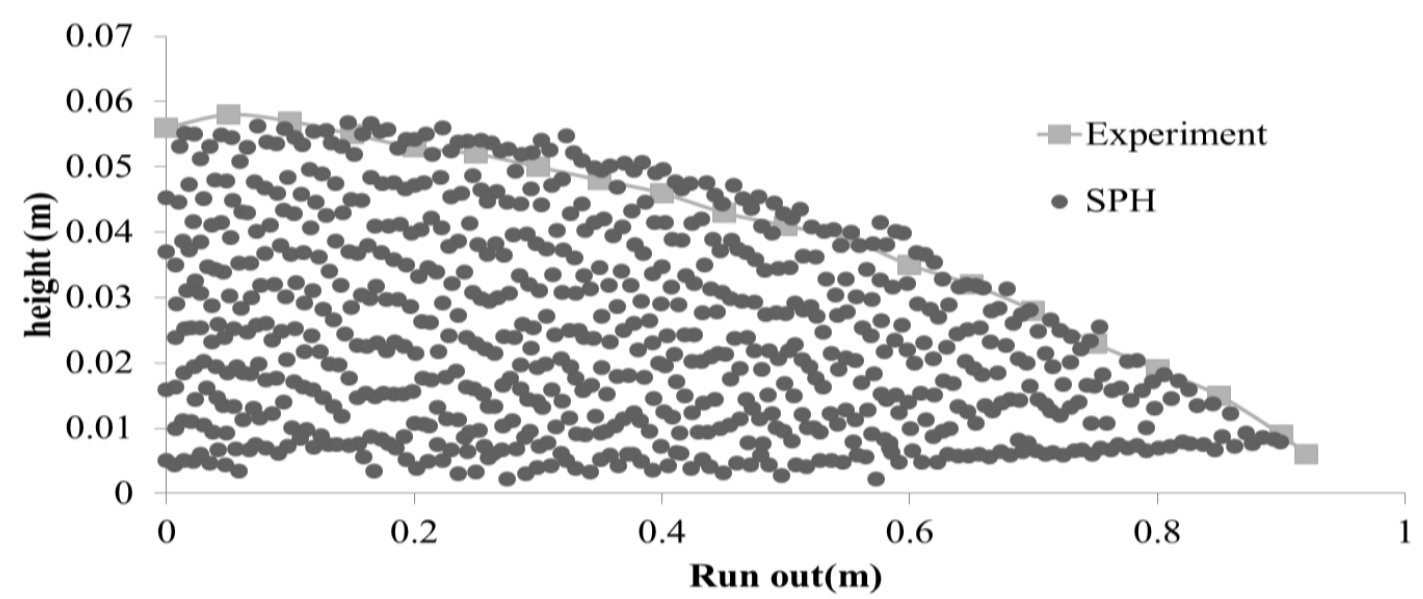

Figure 5.17: The final geometry of the fluid for test \#3.

\section{c. Transient flow prediction for a single layer}

Henriquez and Simms (2009) compared the flow profiles obtained from experimental tests to profiles predicted by using the equations presented by Yuhi and Mei (2004) for one-dimensional transient spreading of a fluid. The deposition is recorded at 3-6-9 seconds after the material is poured through a funnel where the viscosity of the tailings is $0.65 \mathrm{~Pa}$.s where the yield stress is $36 \mathrm{~Pa}$. The results indicated that even though at the beginning there is a good agreement between the predicted and measured profiles however the simulated flow is slightly faster than the recorded one.

The same test applied using SPH and the predictions of the flow at 3-6-9-11 seconds are presented in Figure 5.19. The initial position of the particles for the first part of the simulation ( 3 seconds) is presented in Figure 5.18. Once, all the particles are flown out from the pipe, new set of particles are positioned in the pipe right at the top of the previously flown particles (similar to Fig. 5.16). The initial conditions and the parameters selected for the simulation is summarised in Table 5.4 below. 
One of the main problems encountered in this test is to slowing down the flow rate; in order to overcome this problem the walls of the pipe in the simulations are positioned closer to the particles and the distance between the pipe and the bottom boundary is decreased.

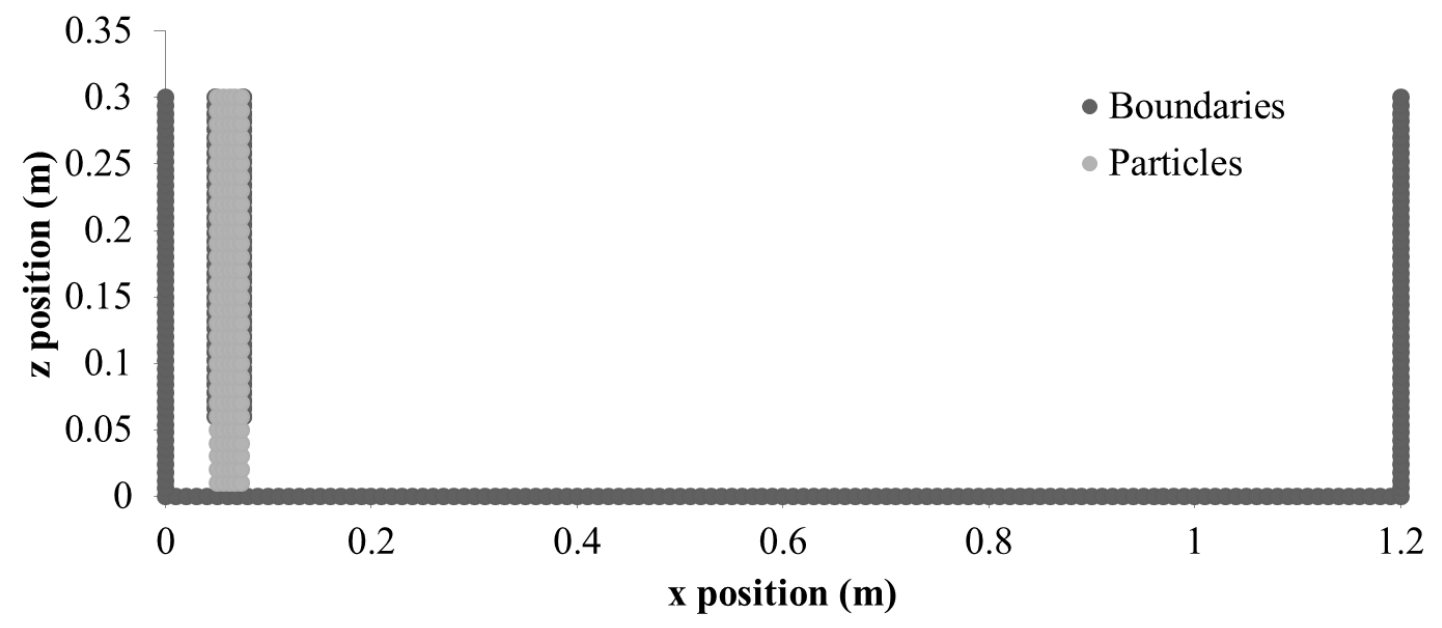

Figure 5.18: The initial boundaries and particle positions for the first part of the simulation ( $3 \mathrm{~s})$

\begin{tabular}{|l|l|l|}
\hline Geometry of the flume & Fluid & Time \\
\hline$d x=0.006 \mathrm{~m}$ & $d x=0.006 \mathrm{~m}$ & $d t=1 \mathrm{E}-06 \mathrm{~s}$. \\
$d z=0.01 \mathrm{~m}$ & $d z=0.01 \mathrm{~m}$ & run time $=11 \mathrm{~s}$. \\
$h=7.27 \mathrm{~mm}$ & $\mathrm{~B}=5000$ & \\
D.B.C & $\alpha=40$ & \\
& $\mu=0.65$ Pa.s & \\
\hline
\end{tabular}

Table 5.4: The parameters selected for test $\# 4$.

The flow profiles predicted by using the proposed model at 3-6-9 seconds are compared with the experimental data obtained in the laboratory. At the earlier stages of the simulations, the agreement between the predicted and measured profiles gives better agreement however the final geometry showed that the simulated flow is slightly faster than the measured data. The main challenge for this specific test was to determine the 
boundary positions in order to slow down the flow rate. The walls are positioned closer to the particles and the distance between bottom boundary and the walls are decreased.

The same test was simulated using the parameters defined in Table 5.3. The positions of the particles are the same; but the distance between the wall and the bottom boundary increased to $0.1 \mathrm{~m}$. Also, the distance between the particles and the walls are slightly increased. The B value is selected as 5000 . The topography seemed to reach the same geometry at 1.3 seconds instead of 3 seconds (Figure 5.20).
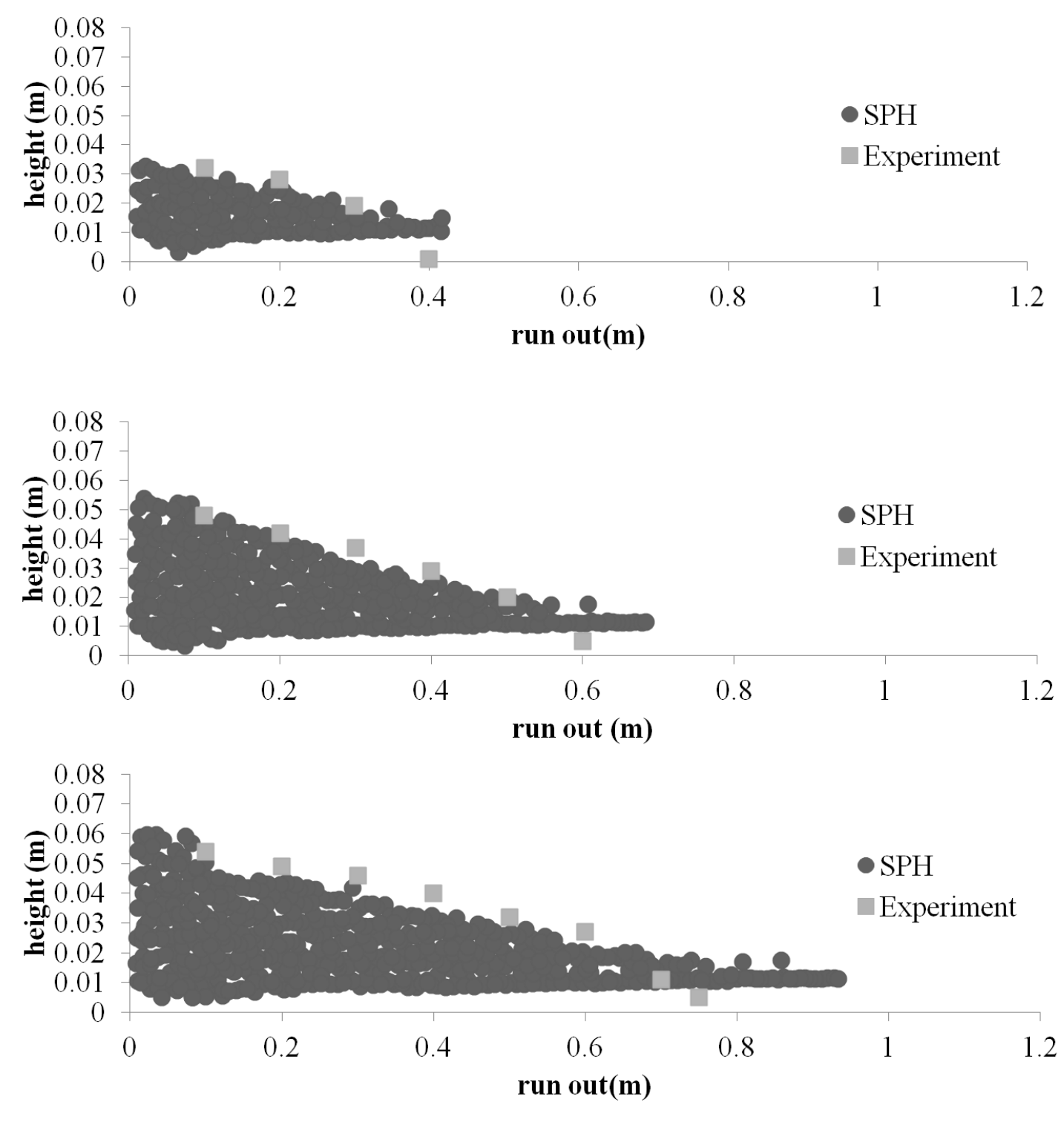


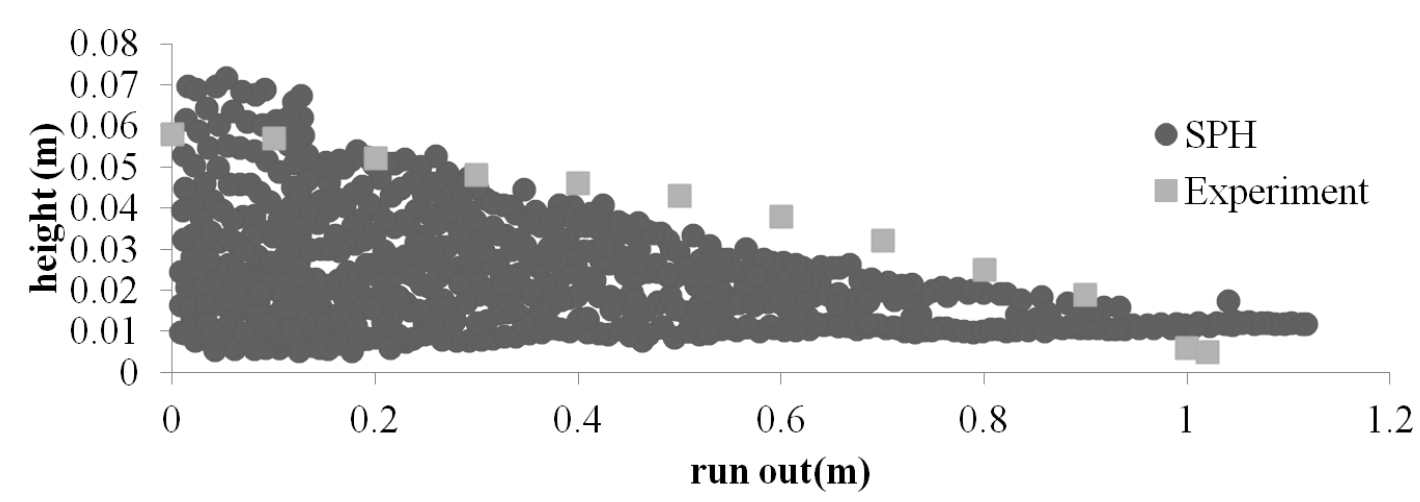

Figure 5.19: Measured and predicted flow profiles at 3-6-9-11 seconds.
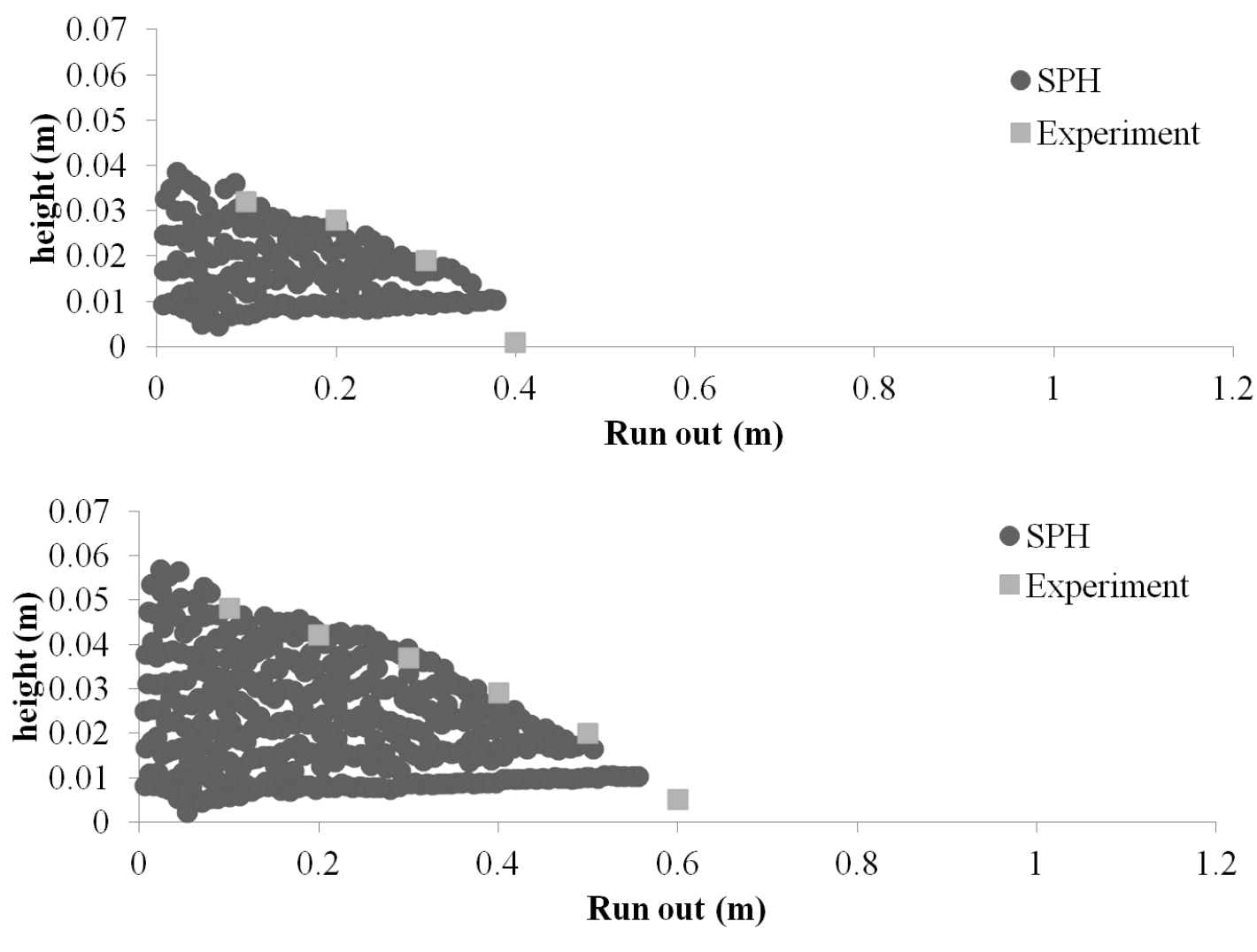

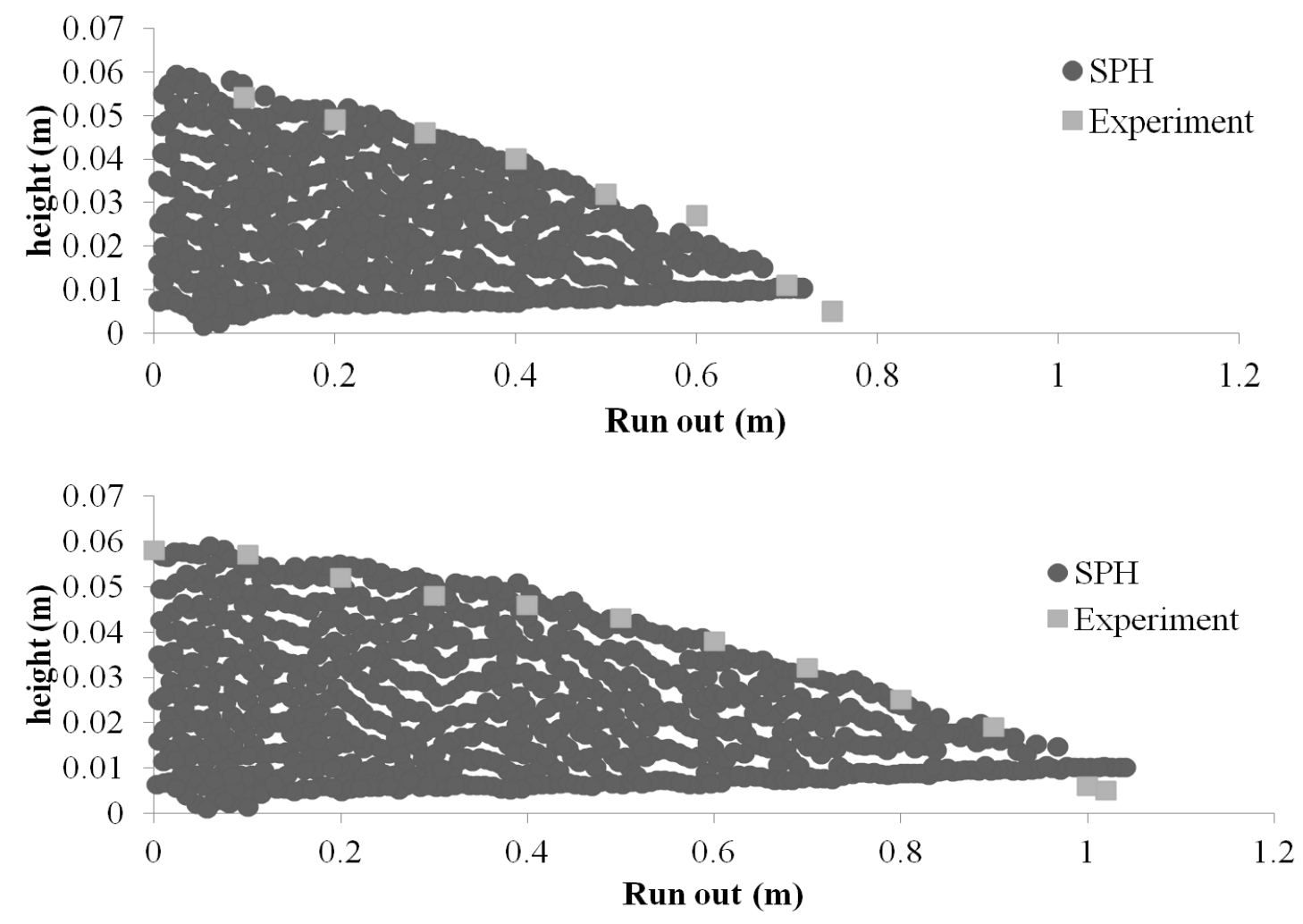

Figure 5.20: Measured and predicted flow profiles for smaller B value at 1.3-2.6-3.9-6.2 seconds.

\section{d. Flume tests on a sloping plane}

The proposed model is also tested for fluid deposited on a sloped plane and compared with the measured data obtained from Henriquez and Simms (2009). Two different angles were used; 0.57 and 1.14 degrees. The results showed good agreement with the experimental tests. The parameters selected for the specific tests are displayed below.

\begin{tabular}{|l|l|l|}
\hline Geometry of the flume & Fluid & Time \\
\hline$d x=0.006 \mathrm{~m}$ & $d x=0.006 \mathrm{~m}$ & $d t=1 \mathrm{E}-06 \mathrm{~s}$. \\
$d z=0.01 \mathrm{~m}$ & $d z=0.01 \mathrm{~m}$ & run time $=2.2 \mathrm{~s}$. \\
$h=5.68 \mathrm{~mm}$ & $\mathrm{~B}=7000$ & \\
D.B.C & $\alpha=40$ & \\
& $\mu=0.65$ Pa.s & \\
\hline
\end{tabular}

Table 5.5: Parameters selected for the flume tests on a sloping bed. 


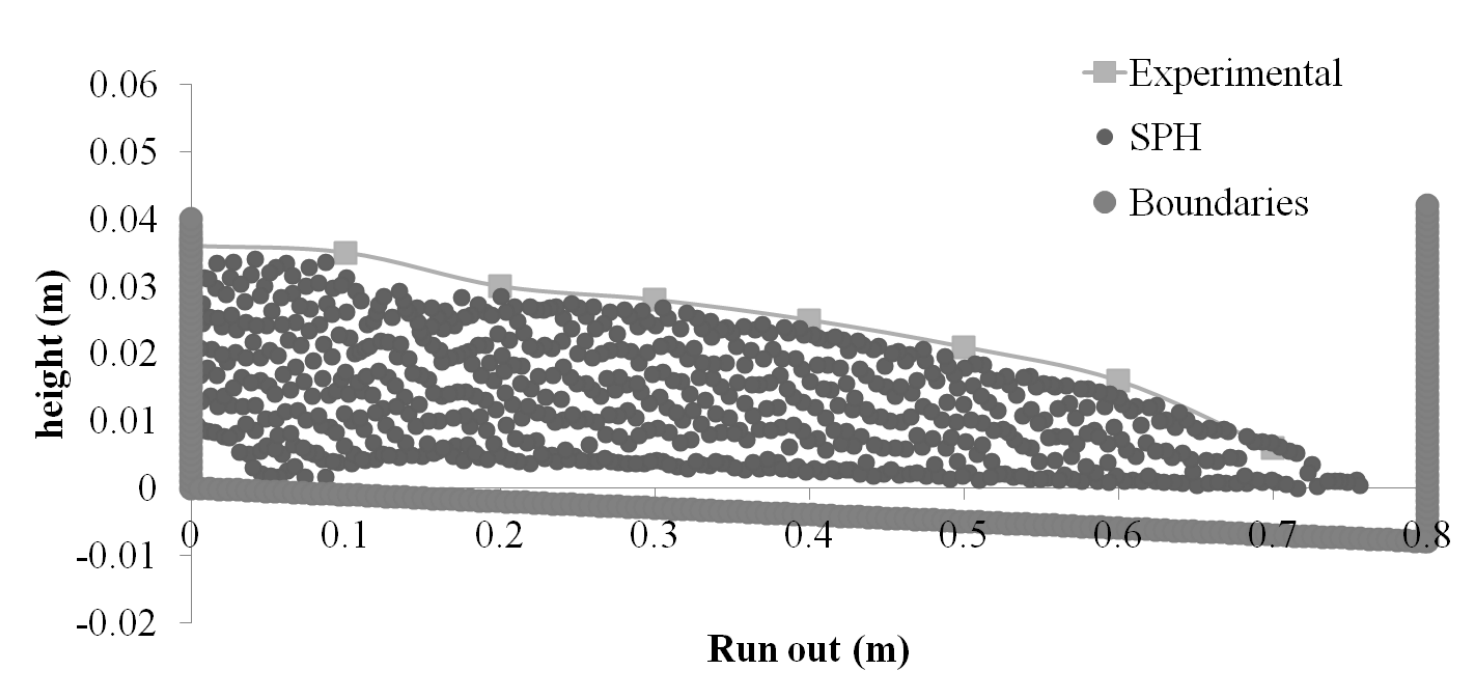

Figure 5.21: Predicted and measured flow profiles at a slope of 0.57 degrees.

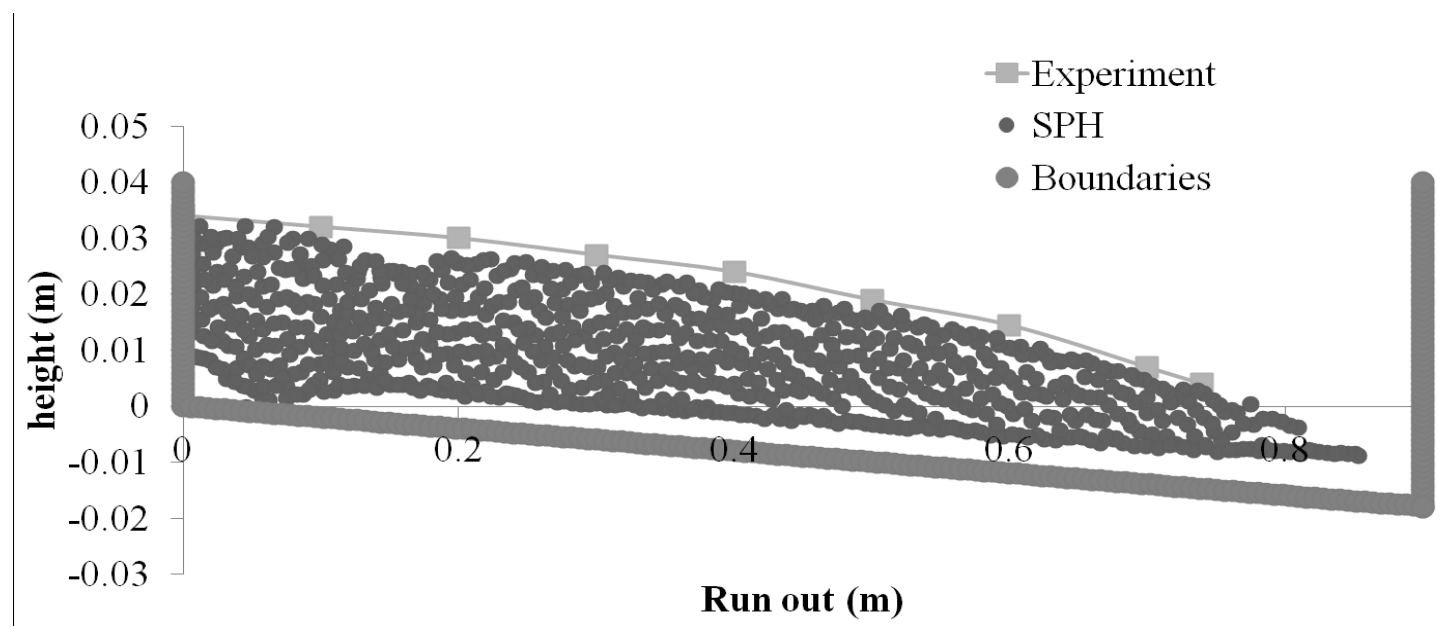

Figure 5.22: Predicted and measured flow profiles at a slope of 1.14 degrees.

\subsubsection{Multi-Layer Flume Test}

As mentioned earlier, numerical model is also tested for flume tests on successive layers. The second layer is poured on top of the existing layer. The results are compared with the experimental data obtained by Henriquez and Simms (2009). The strength of the previous layer must be high enough to support the new deposited layer on top. One of the main challenges in these simulations is to select fitting parameters addressing to this problem; $\mathrm{B}, \mathrm{h}$ and $\alpha$. 
In order to minimise the penetration into the settled layer, the yield stress values selected for the first layer must be high. Several $h, \tau_{\mathrm{y}}$ and $\alpha$ values were tested for multilayer simulations and some of them are displayed in Fig. 5.24. The yield stress values and the viscosity for the new layer are $36 \mathrm{~Pa}$ and $0.65 \mathrm{~Pa}$.s respectively. The calculated yield stress values for the settled layer are considerably higher compared to the new deposited layer; resulting as significant increase in the artificial viscosity value. Selecting a B and $h$ value which is applicable to both layers is challenging.

The initial layer is deposited on top of a horizontal plane and the particle positions are recorded. For the multi-layer simulations, these particle positions are read into the program from an external text file. B value selected for the first layer is 7000 and the smoothing length is $5.68 \mathrm{~mm}$. The final profile of the flow at 1.7 seconds is displayed in Figure 5.23.

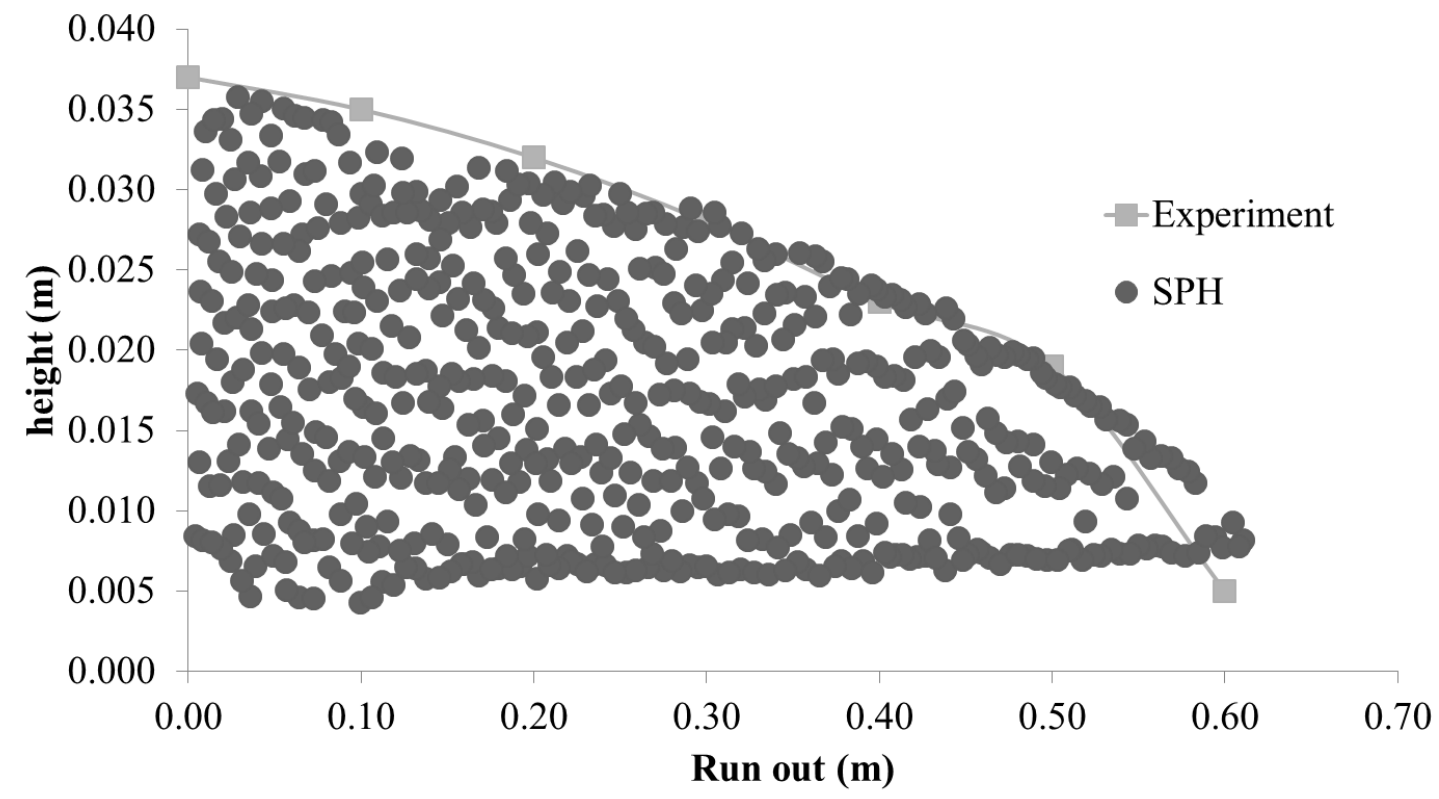

Figure 5.23: The final flow profile for the first layer. 

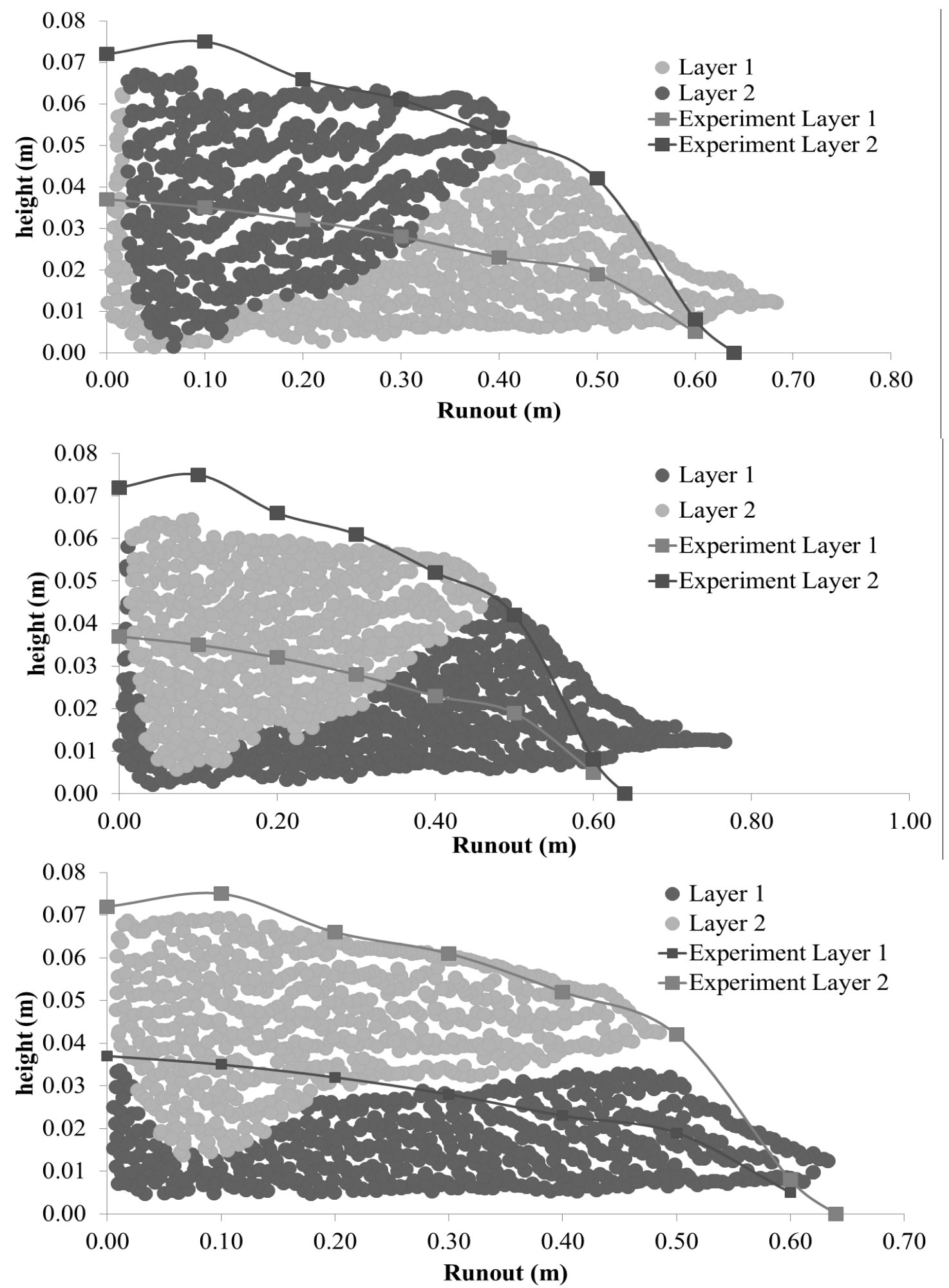


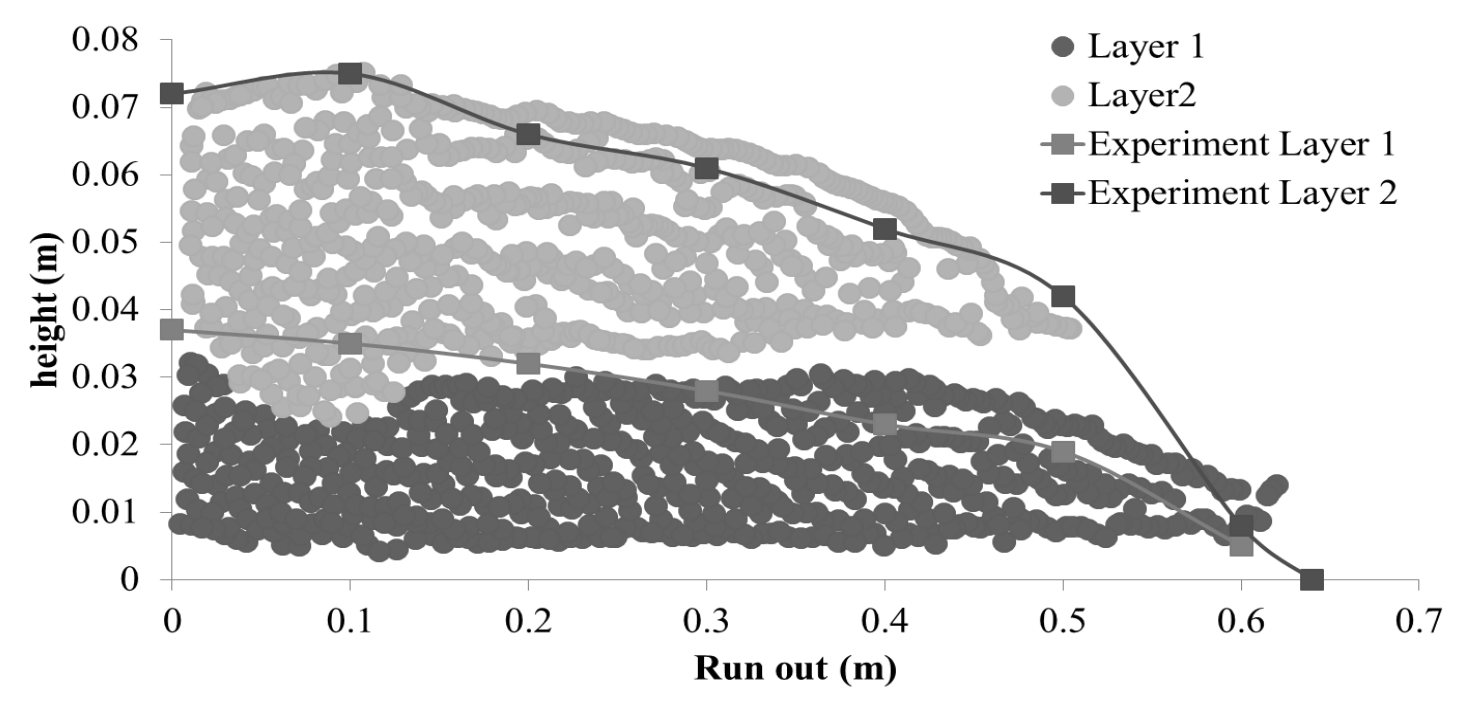

Figure 5.24: Final flow profiles for various $\alpha$ and $\tau_{\mathrm{y}}$ values at 2.4 seconds (a) $\alpha=5000, \tau_{\mathrm{y}}=100$ (b) $\alpha=40$, $\tau_{\mathrm{y}}=400$ (c) $\alpha=5000, \tau_{\mathrm{y}}=400$ (d) $\alpha=50000, \tau_{\mathrm{y}}=1000$

As it can be observed from the figures, the penetration into the settled layer is greater for lower yield stress and alpha values. On the other hand, the gap between the layers, which is caused by the repulsive force calculated between the layers, is smaller for these values. As the values increase, the gap becomes more significant. The best results are achieved (decreasing the penetration) by using the parameters defined in the following table. The initial conditions and the flow profiles are presented in Figure 5.25.

\begin{tabular}{|l|c|c|}
\hline & Geometry of the flume & Fluid \\
\hline Settled Layer & $d x=0.006 \mathrm{~m}$ & $d x=0.006 \mathrm{~m}$ \\
& $d z=0.005 \mathrm{~m}$ & $d z=0.005 \mathrm{~m}$ \\
& $h=1.08 \mathrm{~cm}$ & $\mathrm{~B}=200000$ \\
& $d t=0.01 \mathrm{~s}$. & $\alpha=500$ \\
& & $\tau_{\mathrm{y}}=5000$ \\
& & $\mu=0.65 \mathrm{~Pa} . \mathrm{s}$ \\
\hline New Layer & $d x=0.006 \mathrm{~m}$ & $d x=0.006 \mathrm{~m}$ \\
& $d z=0.005 \mathrm{~m}$ & $d z=0.005 \mathrm{~m}$ \\
& $h=1.08 \mathrm{~cm}$ & $\mathrm{~B}=200000$ \\
& & $\alpha=40$ \\
& & $\tau_{\mathrm{y}}=36$ \\
& & $\mu=0.65 \mathrm{~Pa} . \mathrm{s}$ \\
\hline
\end{tabular}

Table 5.6: Parameters selected for multi-layer simulation. 

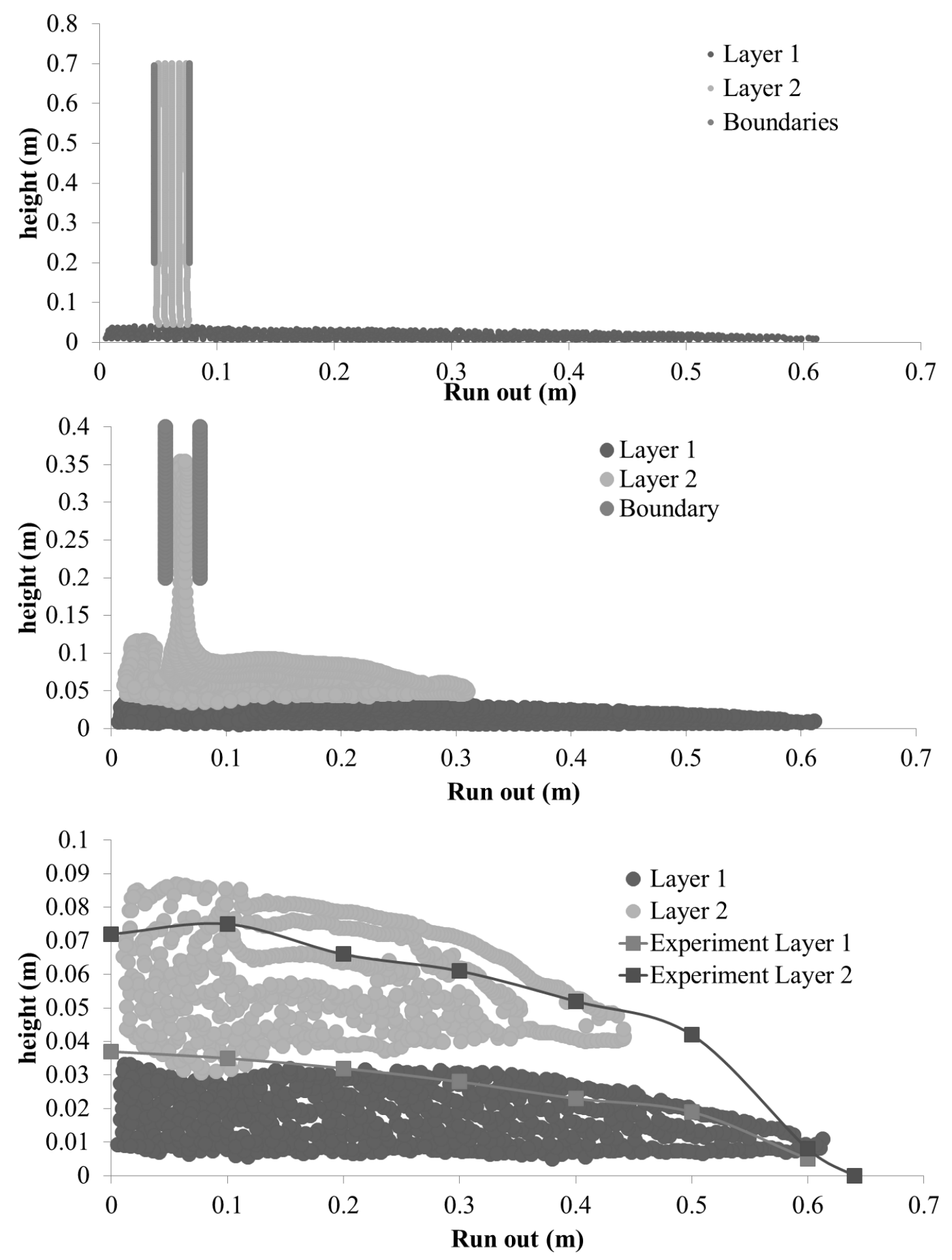


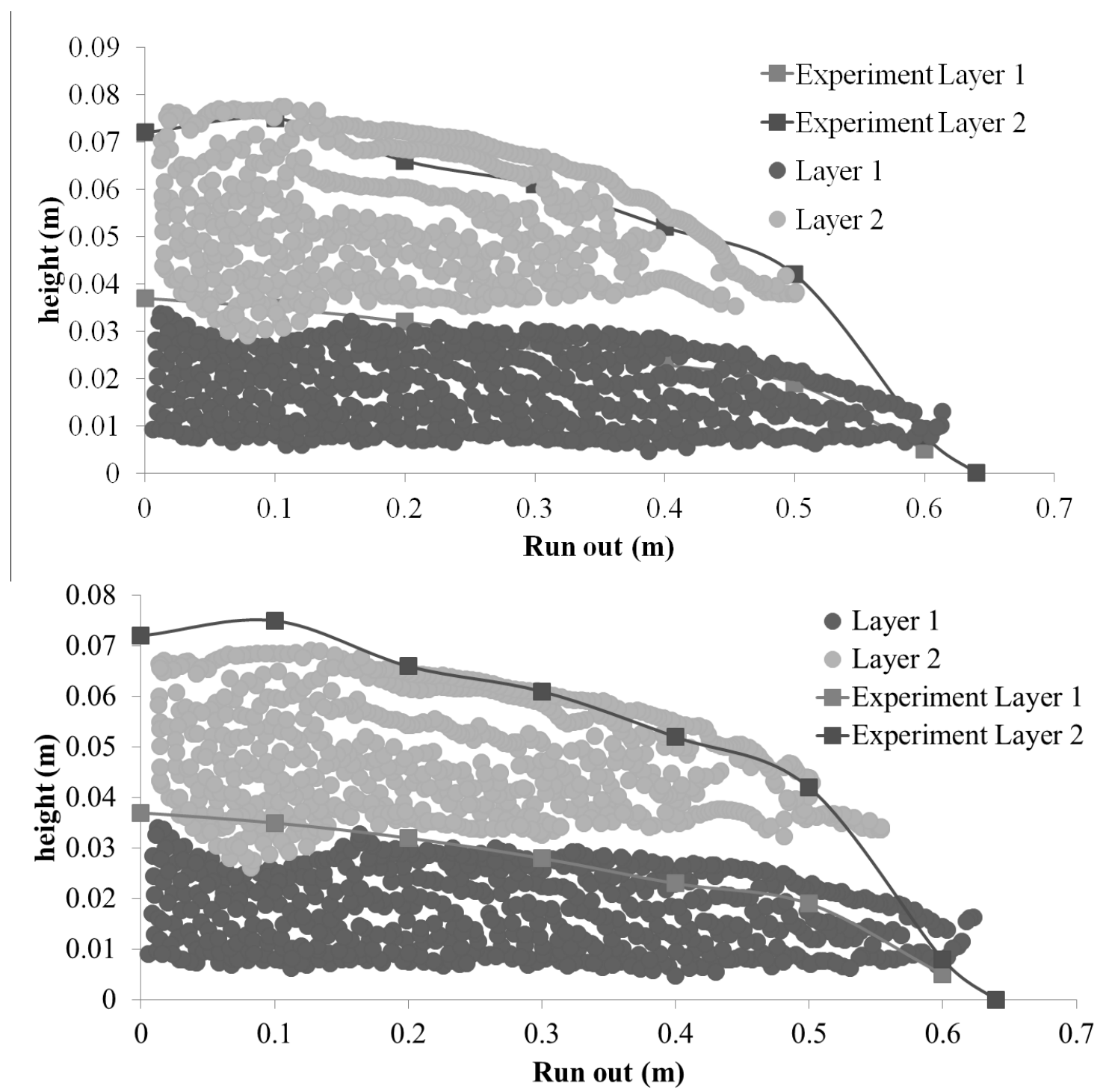

Figure 5.25: Flow profiles at $\mathrm{t}=0.01 \mathrm{~s}, \mathrm{t}=1.0 \mathrm{~s}, \mathrm{t}=2.0 \mathrm{~s}, \mathrm{t}=3.0 \mathrm{~s}, \mathrm{t}=5.0 \mathrm{~s}$

The penetration is minimised with higher yield and alpha values but it still is not completely gone. The average yield stress calculated for the settled later is $15.9 \mathrm{kPa}$ at 3.0 seconds.

The same experiment also conducted using a smaller yield stress and larger $\alpha$ value. The average yield stress is smaller compared to the previous experiment $(9.45 \mathrm{kPa})$ and 
the penetration into the settled layer is greater; however, the gap between the two layers decreases substantially. The selected parameters are presented in the following table.

\begin{tabular}{|l|c|l|}
\hline & Geometry of the flume & Fluid \\
\hline Settled Layer & $d x=0.006 \mathrm{~m}$ & $d x=0.006 \mathrm{~m}$ \\
& $d z=0.005 \mathrm{~m}$ & $d z=0.005 \mathrm{~m}$ \\
& $h=9.68 \mathrm{~mm}$ & $\mathrm{~B}=80000$ \\
& $d t=0.01 \mathrm{s.}$ & $\alpha=50000$ \\
& & $\tau_{\mathrm{y}}=1000$ \\
& & $\mu=0.65$ Pa.s \\
\hline New Layer & $d x=0.006 \mathrm{~m}$ & $d x=0.006 \mathrm{~m}$ \\
& $d z=0.005 \mathrm{~m}$ & $d z=0.005 \mathrm{~m}$ \\
& $h=9.68 \mathrm{~mm}$ & $\mathrm{~B}=80000$ \\
& & $\alpha=40$ \\
& & $\tau_{\mathrm{y}}=36$ \\
& & $\mu=0.65$ Pa.s \\
\hline
\end{tabular}

Table 5.7: Parameters chosen for multi-layer simulation with smaller $\tau_{\mathrm{y}}$ and larger $\alpha$ value.

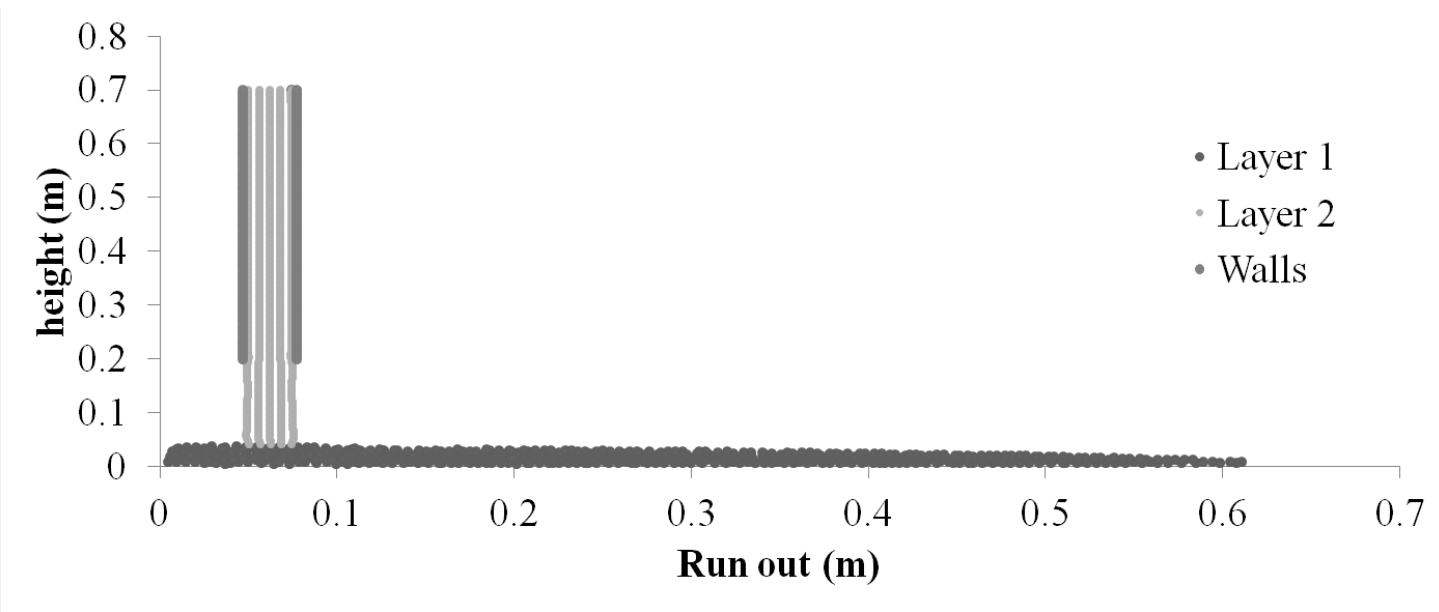



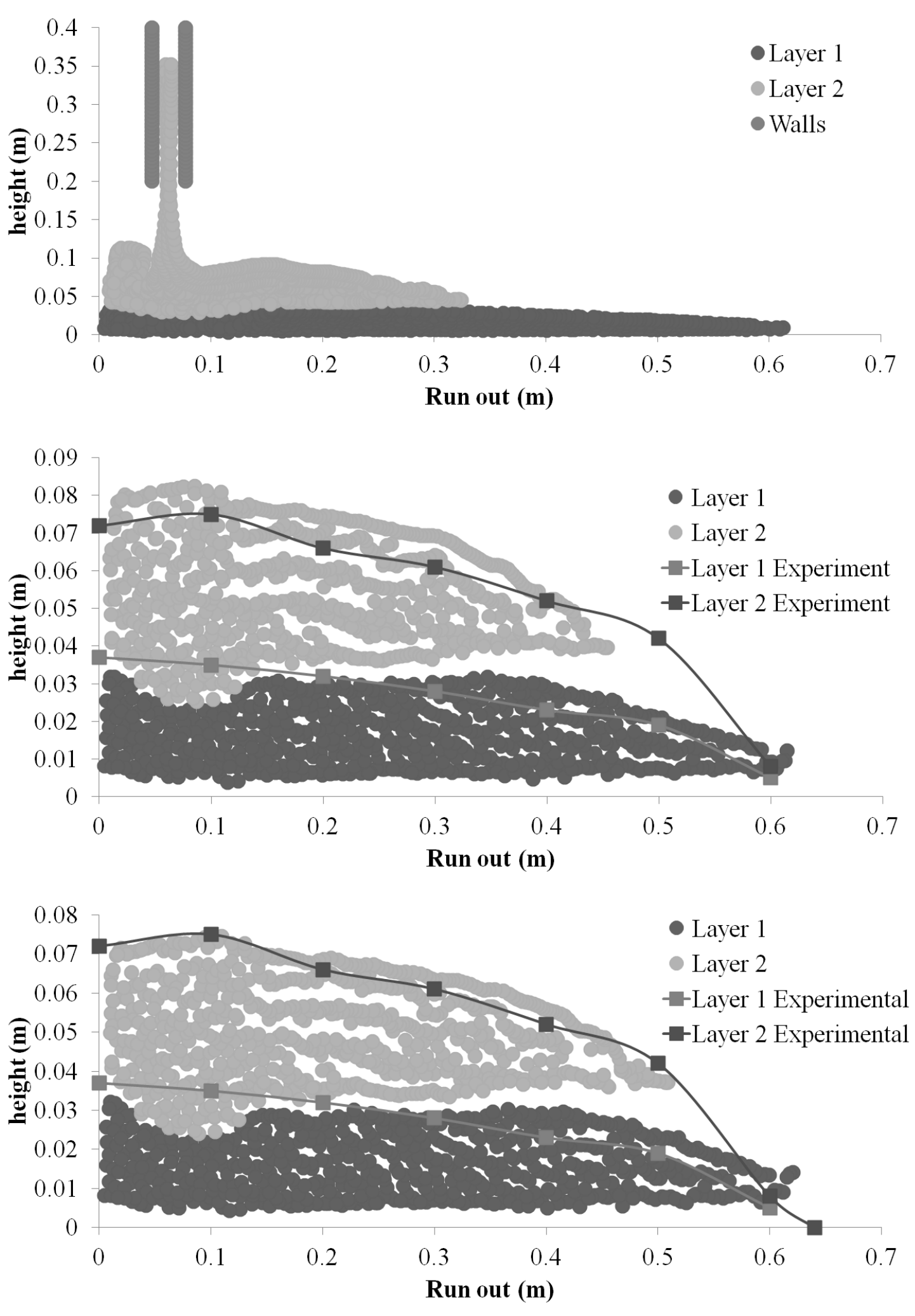


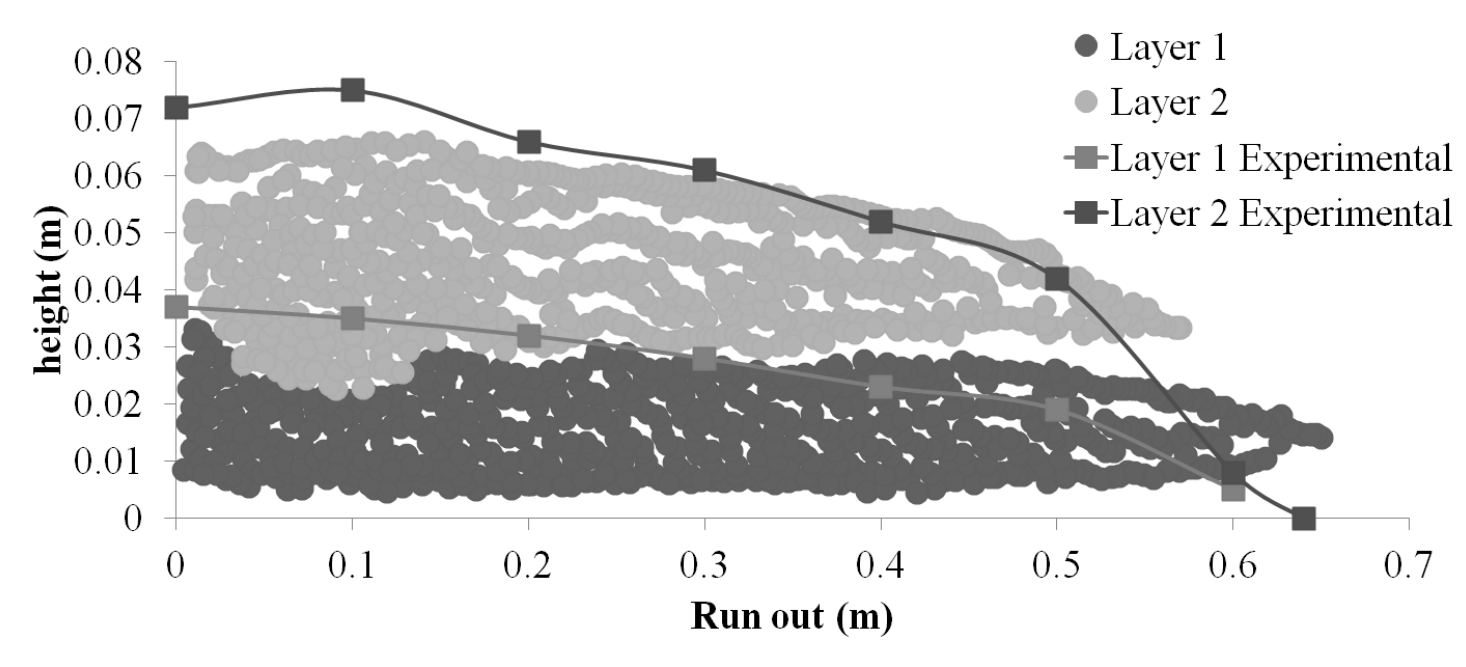

Figure 5.26: Flow profiles at $\mathrm{t}=0.01 \mathrm{~s}, \mathrm{t}=1.0 \mathrm{~s}, \mathrm{t}=2.0 \mathrm{~s}, \mathrm{t}=3.0 \mathrm{~s}, \mathrm{t}=5.0 \mathrm{~s}$. 


\section{Chapter: Conclusion}

Understanding and modelling the flow behaviour is essential to estimate the final geometry of the stack and overall slope angle. This research focuses on the modelling of the depositional behaviour of high density tailings using Smoothed Particle Hydrodynamics method in two dimensions.

To this end, several experimental flume tests were conducted using Bulyanhulu and Val D'Or tailings at laboratory scale where the tailings were deposited with different flow rates and initial water contents. The yield stress values were obtained from slump tests and the results are compared with the Lubrication Theory (LT). Also, LT equations are fitted to the experimental data in order to calculate the best-fitted yield stress values. Finally, three dimensional pouring tests were conducted on $1 \mathrm{~m}$ by $1 \mathrm{~m}$ Plexiglas surface to simulate the flow behaviour of high density tailings in multi layer deposition.

The results from flume tests using Bulyanhulu tailings showed that the deposition flow rate has a significant effect on the final geometry and the overall slope angle. As the deposition time increases, the flow distance becomes smaller resulting in a steeper overall slope angle. Best-fitted yield stress values calculated from LT equations increases significantly with the deposition time. Therefore, for higher deposition rates, final geometry gives a better agreement with Lubrication Theory. Val D'Or tailings used in the experiments are originally conventional tailings with $70 \%$ solid concentration. They are dewatered to a point where they exhibited yield stresses. However, the material used in flume tests and the first two pour tests were collected from the top of the barrel; therefore finer particles dominated the material. The results demonstrated that the Lubrication Theory did not agree well with the experimental data and the best-fitted yield stresses are 
much lower compared to measured yield stresses from slump tests. The same problem also occurred in the first two pour tests. In addition to that, the tailings did not gain enough strength to support the new deposited layers. Therefore, a new barrel was opened and mixed thoroughly and hydrometer tests was applied to several sampled taken from the barrel to make sure the particle size distribution is uniform. The last pour tests were conducted using the new material and the results were more promising. Multi layer experiments showed that the geometry is symmetrical for the first few layers but as the number of layers increase, the flow behaviour of the tailings change and they exhibit convex profiles. As a result, the estimated best fitted yield stress values using are much lower for the upper layers.

The second part of this research is to model the flow behaviour of high density tailings using the Smoother Particle Hydrodynamics (SPH) method. SPH has some advantages over Eulerian grid based methods such as; it provides freedom since the initial condition can be programmed without the need of gridding. On the other hand, the boundary conditions are difficult to implement and the particles must be controlled in order to prevent them to penetrate through the boundaries. Selected time step is usually smaller compared to the other methods which can result in longer computational time. Especially with increasing number of particles, computational effort increases significantly. The model is tested for various initial particle spacing and found that increasing number of particles did not affect the final geometry significantly. The model is validated by comparing the numerical results with the experimental data obtained in the laboratory and from Henriquez and Simms (2009). 
The rheological model chosen is called 'bi-viscosity model' which defines a viscosity value for both yielded and un-yielded regions; therefore the transition between these zones are smoother. The results obtained from the model give good agreement with the experimental data and Lubrication Theory for single layer depositions. Lubrication Theory assumes that the inertial forces negligible; as a result it fails to predict when the inertia influences the flow of tailings. SPH on the other hand, successfully modelled the flow behaviour when the inertial forces affect the flow. The topography of the tailings gives a better correlation with the experimental data for the flume tests conducted using a gate than the Lubrication Theory. For the multi-layer simulations, selected yield stress and alpha values are greater in order to minimise the penetration through the settled layer. However, this generates a greater repulsive force from the settled later resulting a gap between these two layers. This distance become grater as the calculated yield stress from the settled layer increases. In the second multi-layer simulation, the penetration into the settled layer is slightly increased but also the gap between the layers diminishes considerably. 


\section{Chapter: Recommendations}

The simulations provide good agreement with the experimental data in $2 \mathrm{D}$; therefore the next step would be simulating the multi-layer deposition tests in three dimensions specifically to evaluate if SPH is capable of modelling the change in the flow behaviour in the upper layers.

In order to improve the current model, variable $\mathrm{h}$ values might be initially assigned. A constant $\mathrm{h}$ value is selected for the numerical simulations; however the prediction at the edge of the footprint would be more realistic if smaller $\mathrm{h}$ is assigned to the toe. For multi-layer depositions, the current model uses the same $\mathrm{B}$ and $\mathrm{h}$ values for both settled and new layer. However, these values should be different in order to obtain more realistic results.

Controlling the flow rate is one of the substantial difficulties encountered in this research. Other than placing the wall boundaries closer to the particles, the flow rate might be reduced by dividing the volume in the pipe into smaller portions to minimize the effect of inertia. Reducing the flow rate will also positively affect the particle disorder generated in multi-layer depositions. 


\section{Bibliography}

Akcil A, Koldas S. 2006. Acid Mine Drainage (AMD): causes, treatment and case studies. Journal of Cleaner Production:Pages 1139-1145.

Annual Book of ASTM Standards. Designation: D 422-63 Standard test method for particle-size analysis of soils, Philadelphia, PA, 1990.

Azam S, Li Q. 2010. Tailings dam failures: a review of the last one hundred years. Geotechnical News 28(4):50-54.

Balhoff M, Sanchez-Rivera D, Kwok A, Mehmani Y, Prodanović M. 2012. Numerical algorithms for network modeling of yield stress and other non-Newtonian fluids in porous media. Transport in porous media 93(3):363-379.

Balmforth N, Craster R, Sassi R. 2002. Shallow viscoplastic flow on an inclined plane. Journal of Fluid Mechanics 470:1-29.

Beverly C, Tanner R. 1992. Numerical analysis of three-dimensional Bingham plastic flow. Journal of non-newtonian fluid mechanics 42(1):85-115.

Bird RB, Stewart WE, Lightfoot EN. 2007. Transport phenomena: Wiley. com.

Bovet E, Preziosi L, Chiaia B, Barpi F. The level set method applied to avalanches; 2007. p 321-325.

Capone T. 2009. SPH numerical modelling of impulse water waves generated by landslides: Master's thesis, Sapienza University of Rome.

Capone T, Panizzo A, Monaghan JJ. 2010. SPH modelling of water waves generated by submarine landslides. Journal of Hydraulic Research 48(S1):80-84.

Cincilla W, Landriault D, Verburg R. Application of paste technology to surface disposal of mineral wastes; 1997. p 343-356. 
Colagrossi A, Landrini M. 2003. Numerical simulation of interfacial flows by smoothed particle hydrodynamics. Journal of Computational Physics 191(2):448-475.

Crespo AJC. 2008. Application of the Smoothed Particle Hydrodynamics model SPHysics to free-surface hydrodynamics: Ph. D. thesis, University of Vigo.

Crochet MJ, Davies AR, Walters K. 1984. Numerical simulation of non-Newtonian flow: Elsevier Science.

Dean EJ, Glowinski R, Guidoboni G. 2007. On the numerical simulation of Bingham visco-plastic flow: old and new results. Journal of Non-Newtonian Fluid Mechanics 142(1):36-62.

Dzuy NQ, Boger D. 1985. Direct yield stress measurement with the vane method. Journal of Rheology 29:335.

Dzuy NQ, Boger DV. 1983. Yield stress measurement for concentrated suspensions. Journal of Rheology 27:321.

Ellero M, Kröger M, Hess S. 2002. Viscoelastic flows studied by smoothed particle dynamics. Journal of Non-Newtonian Fluid Mechanics 105(1):35-51.

Engman M, Sellgren A, Sundqvist A, Wennberg T, Goldkuhl I. Users perspective on the design of high density base metal tailings handling systems; 2004. Taylor \& Francis. p 45.

Fisseha B, Bryan R, Simms P. 2010. Evaporation, Unsaturated Flow, and Salt Accumulation in Multilayer Deposits of "Paste" Gold Tailings. Journal of geotechnical and geoenvironmental engineering 136(12):1703-1712. 
Fitton T, Chryss A, Bhattacharya S. 2006. Tailings beach slope prediction: a new rheological method. International Journal of Surface Mining, Reclamation and Environment 20(3):181-202.

Fujiyasu Y, Fahey M. 2000. Experimental study of evaporation from saline tailings. Journal of geotechnical and geoenvironmental engineering 126(1):18-27.

Ghosh T, Shook CA. Transport of coarse particles in power law fluids; 1989; Columbia, MO. .

Glowinski R, Wachs A. 2011. On the numerical simulation of viscoplastic fluid flow. Handbook of Numerical Analysis 16:483-717.

Glowinski RW, A. 2011. On the numerical simulation of Viscoplastic flows. . In: P.G. Ciarlet RG, J. Xu, editor. In Handbook of Numerical Analysis. Amsterdam: North-Holland. p pp. 483-719.

Gómez-Gesteira M, Rogers B, Dalrymple R, Crespo A, Narayanaswamy M. 2010. User guide for the SPHysics code v2. 0.

Henriquez J, Simms P. 2009. Dynamic imaging and modelling of multilayer deposition of gold paste tailings. Minerals Engineering 22(2):128-139.

Herard D. 2010. The Hungarian Toxic Red Sludge Spill and Determining Public Accountability.

Hill, Kelly Blue. 1998. Pipeline Flow of Coarse Particles in Fluids with Yield Stresses. Ottawa: National Library of Canada = Bibliothèque Nationale Du Canada, 1998

Hill KB, Shook CA. 1998. Pipeline transport of coarse particles by water and by fluids with yield stresses. Particulate science and technology 16(2):163-183. 
Hosseini S, Manzari M, Hannani S. 2007. A fully explicit three-step SPH algorithm for simulation of non-Newtonian fluid flow. International Journal of Numerical Methods for Heat \& Fluid Flow 17(7):715-735.

Jewell RJ, Fourie AB. 2006. Paste and thickened tailings - A guide. Nedlands, Western Australia: Australian Centre for Geomechanics.

Johnson SB, Franks GV, Scales PJ, Boger DV, Healy TW. 2000. Surface chemistryrheology relationships in concentrated mineral suspensions. International Journal of Mineral Processing 58(1):267-304.

Keslerová R, Kozel K. 2010. Numerical modelling of incompressible flows for Newtonian and non-Newtonian fluids. Mathematics and Computers in Simulation 80(8):1783-1794.

Komatina D, Jovanovic M. 1997. Experimental study of steady and unsteady free surface flows with water-clay mixtures. Journal of Hydraulic Research 35(5):579-590.

Lachamp, P. 2003. Modélisation Numérique De L'effet D'un Obstacle Sur Les écoulements De Fluids à Seuil Par La Méthode SPH. PhD Thesis,. Grenoble, France: Université Joseph Fourier, 2003.

Laigle D, Lachamp P, Naaim M. 2007. SPH-based numerical investigation of mudflow and other complex fluid flow interactions with structures. Computational Geosciences 11(4):297-306.

Liu KF, Mei CC. 1989. Slow spreading of a sheet of Bingham fluid on an inclined plane. J. Fluid Mech 207:505-529.

MAC. 2012. Facts \& Figures 2012 Report. Mining Association of Canada. 
McPhail G, Fourie A, Jewell R, Slatter P, Paterson A. Prediction of the beach profile of high density thickened tailings from rheological and small scale trial deposition data; 2008.

Mizani, Shabnam. Rheology of Thickened Gold Tailings for Surface Deposition. Ottawa: Carleton University, 2010

Mizani S, He X, Simms P. 2013. Application of lubrication theory to modeling stack geometry of high density mine tailings. Journal of Non-Newtonian Fluid Mechanics.

Monaghan J. 2012. Smoothed particle hydrodynamics and its diverse applications. Annual Review of Fluid Mechanics 44:323-346.

Monaghan JJ. 1992. Smoothed particle hydrodynamics. Annual review of astronomy and astrophysics 30:543-574.

Monaghan JJ. 1994. Simulating free surface flows with SPH. Journal of Computational Physics 110(2):399-406.

Monaghan JJ. 2005. Smoothed particle hydrodynamics. Reports on progress in physics 68(8):1703.

Morin, Kevin A., and Nora M. Hutt. "Tailings.info." - Los Frailes Tailings Dam Failure. Minesite Drainage Assessment Group, 7 May 2004. Web. 30 Jan. 2014.

Naef D, Rickenmann D, Rutschmann P, McArdell B. 2006. Comparison of flow resistance relations for debris flows using a one-dimensional finite element simulation model. Natural Hazards and Earth System Science 6(1):155-165.

Pashias N, Boger D, Summers J, Glenister D. 1996. A fifty cent rheometer for yield stress measurement. Journal of Rheology 40:1179. 
Shao S, Lo EY. 2003. Incompressible SPH method for simulating Newtonian and nonNewtonian flows with a free surface. Advances in Water Resources 26(7):787800.

Shook CA, Roco MC. 1991. Slurry flow: principles and practice: ButterworthHeinemann Boston.

Shuttleworth J, Thomson B, Wates J. Surface paste disposal at Bulyanhulu-practical lessons learned; 2005. p 207-218.

Simms P, Dunmola A, Fisseha B, Bryan R. Generic modeling of desiccation for cyclic deposition of thickened tailings to maximize density and to minimize oxidation; 2010. Paste.

Simms P, Grabinsky M, Zhan G. 2007. Modelling evaporation of paste tailings from the Bulyanhulu mine. Canadian Geotechnical Journal 44(12):1417-1432.

Simms P, Williams M, Fitton T, McPhail G, Jewell R, Fourie A. Beaching angles and evolution of stack geometry for thickened tailings-a review; 2011. p 5-7.

Sofrá F, Boger DV. 2002. Environmental rheology for waste minimisation in the minerals industry. Chemical Engineering Journal 86(3):319-330.

Spelay RB. 2007. Solids transport in laminar, open channel flow of non-Newtonian slurries: The University of Saskatchewan.

St-Germain P, Nistor I, Townsend R. 2012. NUMERICAL MODELING OF TSUNAMIINDUCED HYDRODYNAMIC FORCES ON ONSHORE STRUCTURES USING SPH. Coastal Engineering Proceedings 1(33):structures. 81.

Theriault JA, Frostiak J, Welch D. Surface Disposal of Paste Tailings at the Bulyanhulu Gold Mine, Tanzania; 2003; Sudbury. Mining and the Environment. 
Vick SG. 1983. Planing, Design and Analysis of Tailings Dams. New York: John Wiley \& Sons, Inc.

Vola D, Babik F, Latché J-C. 2004. On a numerical strategy to compute gravity currents of non-Newtonian fluids. Journal of computational physics 201(2):397-420.

Yuhi M, Mei CC. 2004. Slow spreading of fluid mud over a conical surface. Journal of Fluid Mechanics 519(25):337-358.

Zhu H, Martys NS, Ferraris C, Kee DD. 2010. A numerical study of the flow of Bingham-like fluids in two-dimensional vane and cylinder rheometers using a smoothed particle hydrodynamics (SPH) based method. Journal of NonNewtonian Fluid Mechanics 165(7):362-375. 


\section{Modifications in SPHYSICS}

\section{A.1 Modifications in Generator Code}

C SUBROUTINE FILL_PART

subroutine fill_part(nn,XXmin,XXmax,YYmin,YYmax,ZZmin,ZZmax, g,dx,dy,dz,expont,beta,theta)

include 'common.gen2D'

double precision $\mathrm{x} 1, \mathrm{z} 1, \mathrm{XXmin} \_$initial

!The additional section in the subroutine

write $(*, *)$ ' Number of particles from previous simulation ??'

$\operatorname{read}(*, *)$ numb

write $(*, *)$ numb !if the total volume is divided into smaller sections

!The particle positions and velocities are added from an external file

write $(*, *)$ ' Number of settled particles ??'

$\operatorname{read}(*, *)$ numsett

write $(*, *)$ numsett !Reads the particle positions for the first layer.

!Only applicable to multi-layer simulations

$\operatorname{open}(103$, file $=$ 'previous.txt')

open $(101$, file $=$ 'xprevious.txt')

open(102,file='zprevious.txt')

do num $=1$, numb

$\mathrm{nn}=\mathrm{nn}+1$

$\operatorname{read}\left(103,{ }^{*}\right) \times x(n n), z p(n n), u p(n n), w p(n n), r h o p(n n), p(n n), p m(n n)$

enddo

do num $=1$,numsett

$\mathrm{nn}=\mathrm{nn}+1$ 


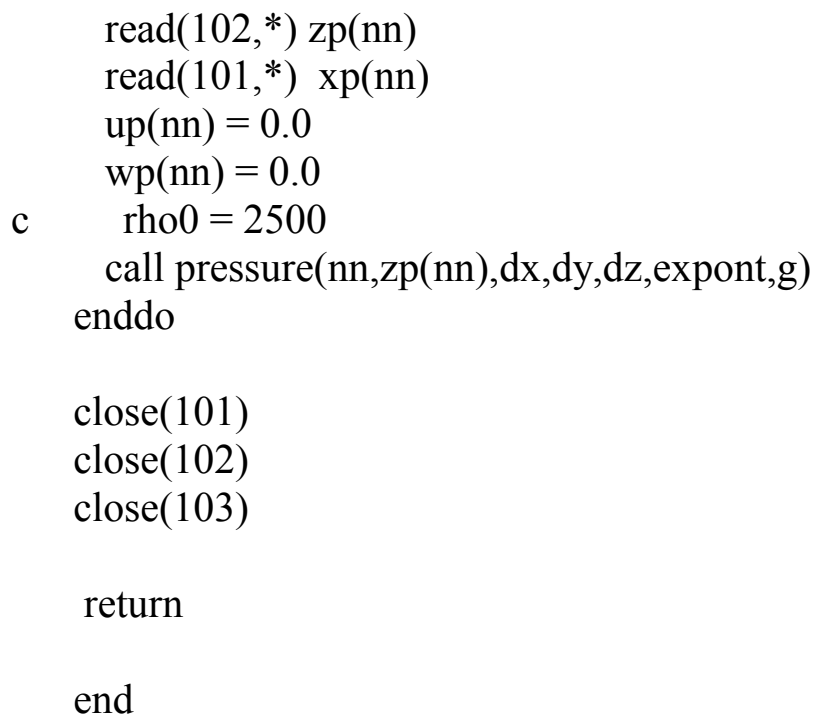




\section{A.2 Modifications in SPHYSICS Code}

subroutine correct

include 'common.2D'

$\mathrm{c}$

c ... account for body forces

$\mathrm{c}$

do $\mathrm{i}=\mathrm{nbp} 1, \mathrm{np}$

$\operatorname{udot}(i)=\operatorname{udot}(i)+\operatorname{grx} * \operatorname{iflag}(i)$

$w \operatorname{dot}(i)=w \operatorname{dot}(i)+\operatorname{grz} *$ iflag(i)

enddo

c ... account for $\mathrm{XSPH}$

$\mathrm{c}$

do $\mathrm{i}=\mathrm{nbp} 1$,np

$$
\begin{aligned}
& x \operatorname{dot}(i)=u p(i)+x \operatorname{cor}(i) \\
& \operatorname{zdot}(i)=w p(i)+z \operatorname{cor}(i)
\end{aligned}
$$

enddo

! New layer

do $\mathrm{i}=$ nbp1,nset

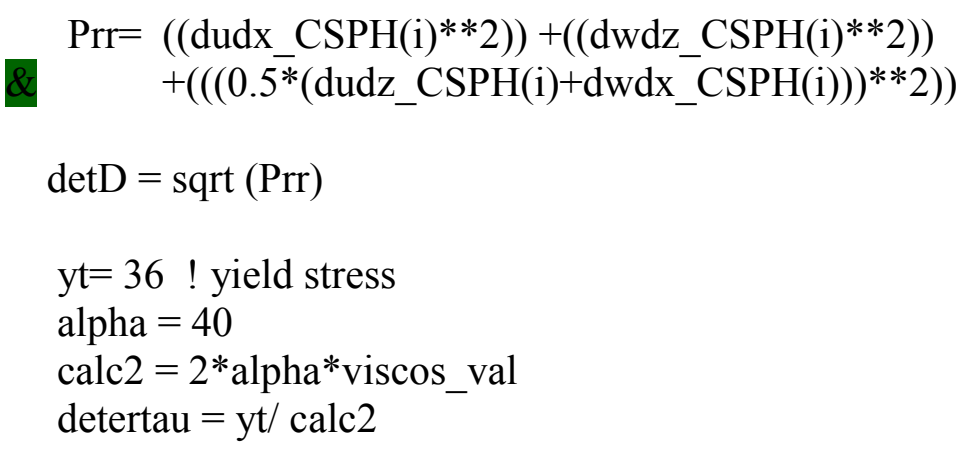




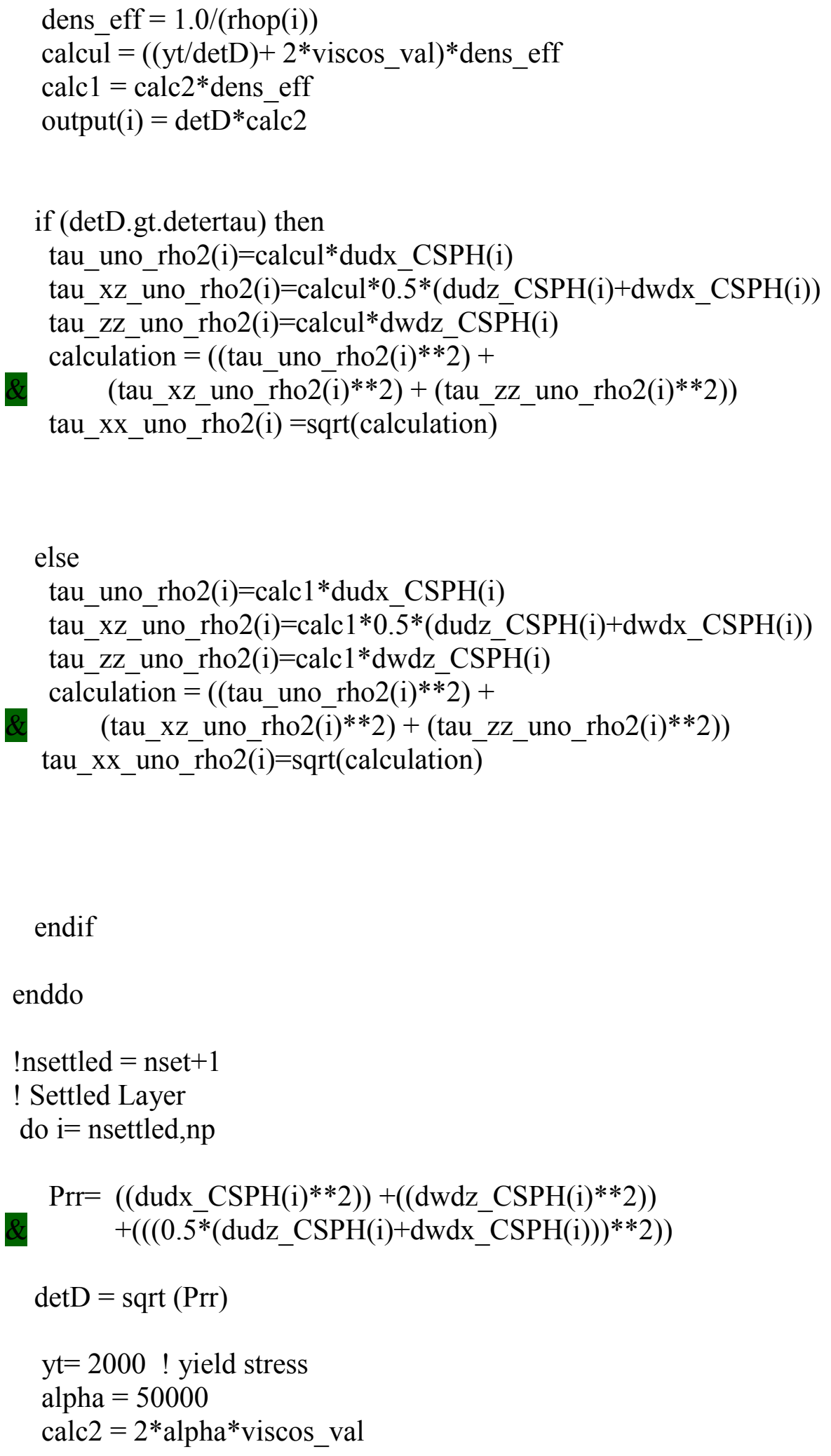




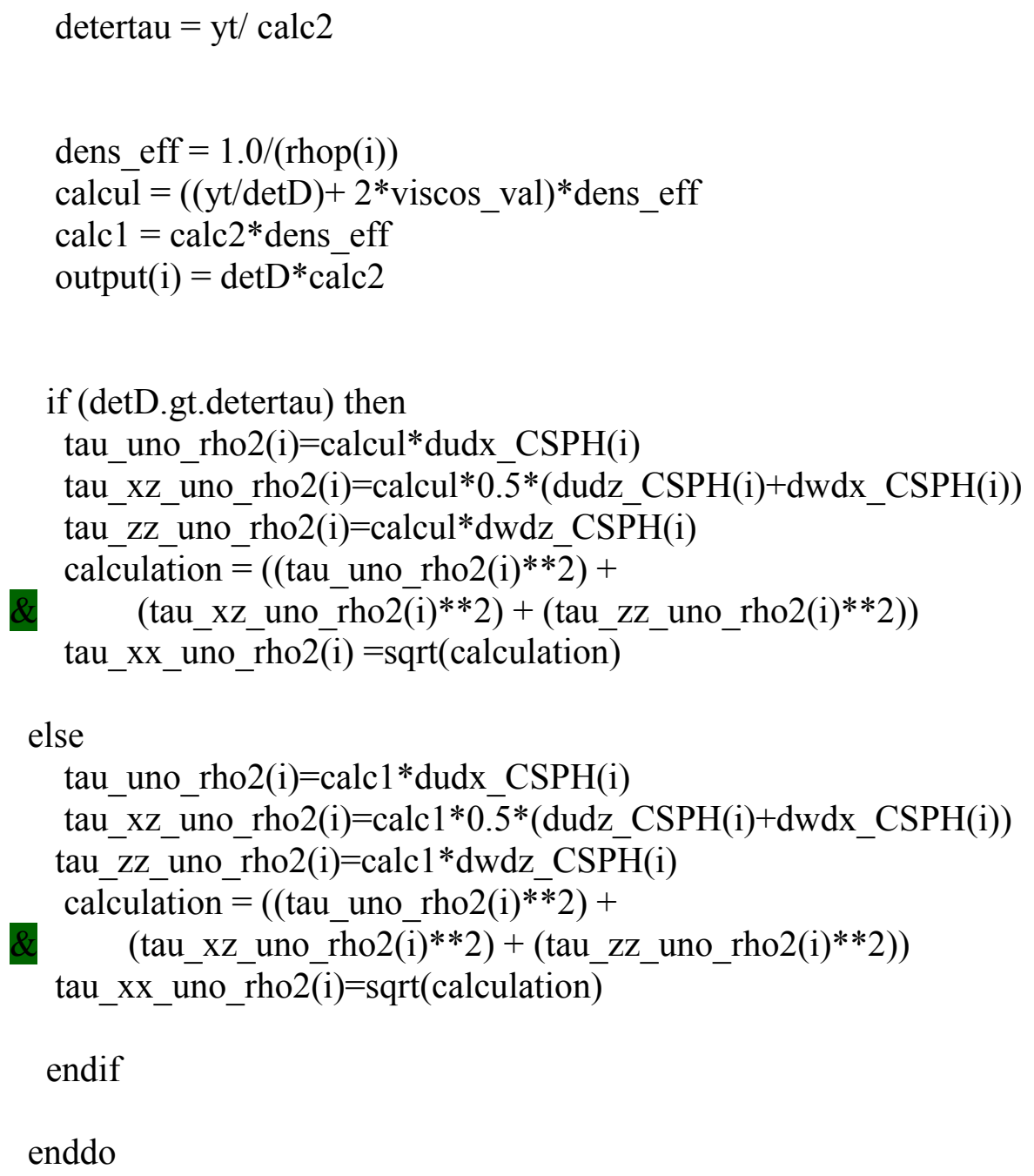




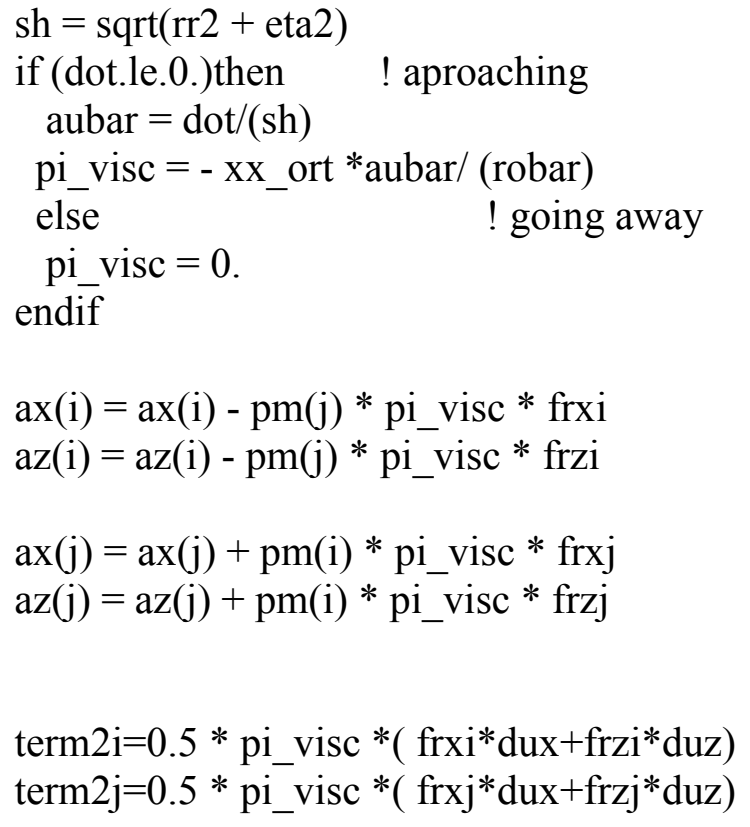

return

end 